\author{
UNIVERSIDADE DE SÃO PAULO \\ ESCOLA DE ARTES, CIÊNCIAS E HUMANIDADES \\ PROGRAMA DE PÓS-GRADUAÇÃO EM GERONTOLOGIA
}

ALINE CRISTINA DA SILVA

Avaliação em idosos: uma investigação sobre instrumentos de medida

São Paulo 


\section{Avaliação em idosos: uma investigação sobre instrumentos de medida}

Dissertação apresentada à Escola de Artes, Ciências e Humanidades da Universidade de São Paulo para obtenção do título de Mestre em Ciências pelo Programa de Pós-graduação em Gerontologia.

Versão corrigida contendo as alterações solicitadas pela comissão julgadora em 16 de março de 2021. A versão original encontra-se em acervo reservado na Biblioteca da EACH/USP e na Biblioteca Digital de Teses e Dissertações da USP (BDTD), de acordo com a Resolução CoPGr 6018, de 13 de outubro de 2011.

Área de Concentração:

Gerontologia

Orientador:

Prof. Dr. Flávio Rebustini 
Autorizo a reprodução e divulgação total ou parcial deste trabalho, por qualquer meio convencional ou eletrônico, para fins de estudo e pesquisa, desde que citada a fonte.

Silva, Aline Cristina da

Avaliação em idosos: uma investigação sobre instrumentos de medida / Aline Cristina da Silva ; orientador, Flávio Rebustini. - 2021.

$$
164 \mathrm{f} \text { : il. }
$$

Dissertação (Mestrado em Ciências) - Programa de PósGraduação em Gerontologia, Escola de Artes, Ciências e Humanidades, Universidade de São Paulo, São Paulo Versão corrigida

1. Idosos - Avaliação - Brasil. 2. Psicometria. 3. Avaliação em saúde. I. Rebustini, Flávio, orient. II. Título

CDD 22.ed. - 362.60981 
SILVA, Aline Cristina da

Avaliação em idosos: uma investigação sobre instrumentos de medida

Dissertação apresentada à Escola de Artes, Ciências e Humanidades da Universidade de São Paulo para obtenção do título de Mestre em Ciências do Programa de Pós-Graduação em Gerontologia.

Área de Concentração:

Gerontologia

Aprovado em: 16 / 03 / 2021

\section{Banca Examinadora}

\begin{tabular}{llll} 
Prof. Dr. & Afonso Antonio & Instituição: & UNESP \\
& Machado & & \\
Julgamento: & Aprovada & Assinatura: & \\
\cline { 3 - 3 } & & & \\
Prof. Dr. & Ruth Caldeira de Melo & Instituição: & EACH - USP \\
Julgamento: & Aprovada & Assinatura: & \\
& & & \\
Prof. Dr. & Evandro Morais Peixoto & Instituição: & UPE \\
Julgamento: & Aprovada & Assinatura: &
\end{tabular}


Dedico a todos aqueles que compartilharam comigo essa árdua e bela jornada. 


\section{Agradecimentos}

A chegada sempre diz respeito à gratidão...

Agradeço primeiramente à minha família, que foi fonte de energia e ânimo para que mais este passo se concretizasse.

À Daniel Cimento, meu companheiro, que sempre apoiou minhas decisões e acreditou nos meus sonhos, contribuindo incessantemente para que eles se tornassem realidade.

Ao meu filho, Miguel Cimento, que aos 40 dias de vida, já me apoiava nessa caminhada, com cada sorriso, mesmo sem saber.

Aos meus pais, Nanci e Wagner, que sempre vibraram e se orgulharam à cada passo em frente.

Ao meu irmão mais novo, Renan, que sempre ofereceu ouvidos para partilhar as angústias e alegrias.

Ao meu querido orientador e amigo, Professor Doutor Flávio Rebustini, que confiou, apoiou e suportou todos os momentos dessa jornada, mesmo antes que ela começasse. Acreditando no meu potencial e sempre oportunizando entregas que nem eu mesma acreditava ser possível.

Aos Professores Doutores, membros da minha banca de qualificação e defesa, Doutor Afonso Antonio Machado, Doutora Ruth Caldeira de Melo e Doutor Evandro Morais Peixoto, que se disponibilizaram e dedicaram a ler, compreender, contribuir e participar neste importante momento dessa jornada.

Aos meus amigos incríveis, fruto da graduação, Professores e Mestres Bruno Bonfá Araujo e Geovana Mellisa Castrezana Anacleto, por todo apoio, incentivo, lembranças e risadas nas apresentações em congressos, nas trocas diárias e discussões infinitas sobre a ciência e sobre o mundo. Foram essenciais para eu chegar aqui!

A todos os professores do Programa de Pós-Graduação em Gerontologia da EACHUSP, ao qual me proporcionaram reflexões nunca ainda possíveis, sobre o universo da gerontologia e da pós-graduação.

Às queridas amigas e divas da minha turma, Dhiene, Carina e Karina, que foram ótimas parceiras de aulas e de trocas da vida.

A chegada é sempre gratificante, mas a jornada é espetacular e deve ser aproveitada a cada instante, muito obrigada por fazerem parte dela! 
"Que os vossos esforços desafiem as impossibilidades. Lembrai-vos de que as grandes proezas da história foram conquistadas do que parecia impossível." (CHAPLIN, Charles) 


\section{RESUMO}

SILVA, Aline Cristina da. Avaliação em idosos: uma investigação sobre instrumentos de medida. 2021. 164 f. Dissertação (Mestrado em Ciências) - Escola de Artes, Ciências e Humanidades, Universidade de São Paulo, São Paulo, 2021. Versão corrigida.

A avaliação em idosos ainda enfrenta muitos desafios, compreender os instrumentos de medida que fazem parte deste processo, é parte essencial para avanço, bem como para a própria Gerontologia. Portanto, o objetivo desta dissertação foi organizar evidências dos instrumentos psicométricos disponíveis para idosos brasileiros, disponibilizando à estudiosos, profissionais, pesquisadores e à própria população e familiares, insumos para avaliações cada vez mais consistentes, eficientes e eficazes, independente do seu objetivo-fim. No primeiro artigo, por meio de uma scoping review, realizou-se um mapeamento dos instrumentos, buscando estudos de evidências psicométricas disponíveis na literatura nacional e internacional, que foram categorizados de forma sistematizada, oportunizando a apresentação destes de maneira organizada, de acordo com sua variável latente e outras características pertinentes. O segundo estudo, uma revisão sistemática das propriedades psicométricas dos estudos de evidências de validade, identifica e analisa estas evidências. No primeiro estudo, foi possível identificar 192 instrumentos, nos 237 estudos recuperados. Os dados extraídos foram analisados e dispostos em três blocos. O primeiro bloco tratou dos "identificadores da revisão", o segundo apresentou os dados sobre as amostras envolvidas e o terceiro identificou as propriedades psicométricas recuperadas. No segundo artigo desta dissertação, identificou-se 25 instrumentos nos 37 estudos recuperados. Estes instrumentos se propõem a medir as variáveis: ansiedade, depressão e estresse. Os estudos foram analisados e apresentados em três etapas. A primeira etapa refere-se à comunicação das evidências recuperadas, caracterização das amostras e dos instrumentos, a segunda tratou de verificar a qualidade metodológica dos estudos analisados, por meio de um protocolo adaptado do COSMIN, e por fim, a terceira etapa, apresentou critérios e indicadores para a análise dos procedimentos percorridos na busca das evidências de validade. As áreas/campos da Gerontologia e Geriatria e todas as outras interessadas, contam hoje com um arsenal de instrumentos disponíveis e descritos como validados na maioria dos estudos disponíveis. Entretanto, a aplicação do estado da arte contemporâneo das evidências de validade e estudos mais completos qualitativamente ainda são necessários. Visto que a Gerontologia é um campo multidisciplinar crescente e necessita cada vez mais de especialistas com prontidão às temáticas que se relacionam com seus desafios, mas também com o que pode ser fonte de 
melhorias ao seu objeto de estudo, é eminente o reconhecimento deste cenário e aprofundamento destas questões. Especificamente sobre os instrumentos de medida para avaliação de ansiedade, depressão e estresse, para idosos brasileiros, nota-se a carência de desenvolvimento local/regional, visto as controvérsias de tradução/adaptação transcultural de versões estrangeiras. Muito se tem a explorar sobre as determinações amostrais e se atentar as diferentes versões encontradas na literatura sobre o mesmo instrumento. Aos pesquisadores interessados na ciência psicométrica, fica o convite ao exercício da melhoria contínua em relação à atualização constante do conhecimento sobre novas teorias e técnicas estatísticas/psicométricas para escolhas mais assertivas, bem como de fato utilizar os novos conceitos em estudos ao qual participa, possibilitando estudos mais fidedignos, por meio de uma comunicação mais inteligível e inclusiva. A ciência se faz no questionamento diário e isso é permanente e comum para qualquer campo/área.

Palavras-chave: Gerontologia. Psicometria. Brasil. Instrumentos. Avaliação. 


\begin{abstract}
SILVA, Aline Cristina da. Evaluation in the elderly: in investigation into measurement instruments. 2021. 164 p. Dissertation (Master of Scince) - Escola de Artes, Ciências e Humanidades, Universidade de São Paulo, São Paulo, 2021. Corrected Version.
\end{abstract}

Evaluation in the elderly still faces many challenges, understanding the measurement instruments that are part of this process is an essential part for advancement, as well as for Gerontology itself. Therefore, the objective of this dissertation was to organize evidence of the psychometric instruments available to Brazilian elderly people, making available to scholars, professionals, researchers and the population itself and family members, inputs for increasingly consistent, efficient and effective assessments, regardless of their end-objective. In the first article, through a scoping review, a mapping of the instruments was carried out, looking for studies of psychometric evidence available in national and international literature, which were categorized in a systematic way, allowing the presentation of these in an organized manner, according to their latent variable and other relevant characteristics. The second study, a systematic review of the psychometric properties of validity evidence studies, identifies, verifies and analyzes this evidence. In the first study, it was possible to identify 192 instruments, in the 237 studies retrieved. The extracted data were analyzed and organized in three blocks. The first block dealt with the "review identifiers", the second presented the data on the samples involved and the third identified the recovered psychometric properties. In the second article of this dissertation, 25 instruments were identified in the 37 studies retrieved. These instruments aim to measure the variables: anxiety, depression and stress. The studies were analyzed and presented in three stages. The first stage refers to the communication of the recovered evidence, characterization of the samples and instruments, the second attempted to verify the methodological quality of the studies analyzed, through a protocol adapted from COSMIN, and finally, the third stage, presented criteria and indicators for the analysis of the procedures followed in the search for evidence of validity. The areas / fields of Gerontology and Geriatrics and all other stakeholders today have an arsenal of instruments available and described as validated in most of the available studies. However, the application of contemporary state of the art evidence of validity and more complete qualitative studies are still needed. Since Gerontology is a growing multidisciplinary field and needs more and more specialists with readiness for the themes that relate to the challenges, but also with what can be a source of improvements to its object of study, the recognition of this scenario is imminent and deepening 
these issues. Specifically on the measurement instruments for assessing anxiety, depression and stress, for elderly Brazilians, there is a lack of local / regional development, given the controversies of translation / cross-cultural adaptation of foreign versions. Much has to be explored on sample determinations and the different versions found in the literature on the same instrument are taken into account. Researchers interested in psychometric science are invited to exercise continuous improvement in relation to constantly updating knowledge about new theories and statistical / psychometric techniques for more assertive choices, as well as actually using the new concepts in studies in which they participate, enabling more reliable studies, through a more intelligible and inclusive communication. Science is done in daily questioning and this is permanent and common for any field / area.

Key-words: Gerontology. Psychometry. Brazil. Assessment. Evaluation. 


\section{LISTA DE ABREVIATURAS E SIGLAS}

$\mathrm{ACC}$

$\mathrm{ACP}$

ADP

AERA

AFC

AFE

AGA

AIC

AM

ANOVA

APA

ASI

AVC

AVCI

AVD

AVDI

BARTLETT

BHAS

BIC

BP

BVS

C1

$\mathrm{C} 2$

C3

C4

C5

C6

C7

CCL

CES-D

$\mathrm{CE}$
Modelo de Assimilação e Acomodação por Coping

Análise de Componentes Principais

Protocolo de adaptação

Associação Americana de Pesquisa Educacional

Análise Fatorial Confirmatória

Análise Fatorial Exploratória

Avaliação Geriátrica Ampla

Critério de Informação de Akaike

Amostra

Teste para Análise de Variância

Associação Americana de Psicologia

Adult Stressors Inventory

Acidente Vascular Cerebral

Acidente Vascular Cerebral Isquêmico

Atividade de Vida Diária

Atividade Instrumental de Vida Diária

Teste de normalidade dos dados

Bambuí Health Aging Study (estudo longitudinal)

Critério de Informação de Bayes

Benefício Previsto

Biblioteca Virtual da Saúde

Critério 1

Critério 2

Critério 3

Critério 4

Critério 5

Critério 6

Critério 7

Comprometimento Cognitivo Leve

Center for Epidemiologic Studies Depression Scale

Comitê de Especialistas 


\begin{tabular}{|c|c|}
\hline $\mathrm{CF}$ & Confiabilidade \\
\hline CFI & Comparative Fit Index (Índice de Ajuste) \\
\hline COSMIN & $\begin{array}{l}\text { Consensus-Based Standards for the Selection of Health Measurement } \\
\text { Instrument }\end{array}$ \\
\hline $\mathrm{CO}$ & Concorrente \\
\hline $\mathrm{COV}$ & Convergente \\
\hline CSDD & Cornell Scale for Depression in Dementia \\
\hline C_OM & Correlação com Outras Variáveis \\
\hline DA & Doença de Alzheimer \\
\hline DAC & Doença Arterial Coronariana \\
\hline DASS & Depression, Anxiety and Stress Scale \\
\hline DECS & Descritores em Ciências da Saúde \\
\hline DG & Diferença entre Grupos \\
\hline DIF & Differential Item Functioning \\
\hline DIS & Discriminante \\
\hline $\mathrm{DM}$ & Dimensionalidade \\
\hline DP & Doença de Parkinson \\
\hline DP & Desvio Padrão \\
\hline DSE & Depression Scale for the Elderly \\
\hline DSM & Manual Diagnóstico e Estatístico de Transtornos Mentais \\
\hline EBADEP ID & Escala de Baptista de Depressão \\
\hline EMTREE & Embase Subject Headings \\
\hline ENT & Entrevista \\
\hline EPIFLORIPA & $\begin{array}{l}\text { Estudo Sobre as Condições de Vida e Saúde da População Adulta e Idosa } \\
\text { de Florianópolis }\end{array}$ \\
\hline ES & Cálculo do Escore \\
\hline ET & Eye Tracking \\
\hline EX & Técnica de Extração \\
\hline FIBRA & Fragilidade em Idosos Brasileiros (estudo longitudinal) \\
\hline GAD-7 & Generalized Anxiety Disorder Questionnaire \\
\hline GAI-BR & Geriatric Anxiety Inventory \\
\hline GDS-1 & Geriatric Depression Scale (com 1 item) \\
\hline GDS-4 & Geriatric Depression Scale (com 4 itens) \\
\hline
\end{tabular}


GDS-10

GDS-15

GDS-30

GEAP-b

GF

GFI

HADS

HAM-A

HAM-D

HRQol

IC

IC

ICC

ICD-10

ICR

ILPI

IRNI

IV

IVC

JBI

KMO

LSAS

MADRS

MAP

MESH

MINI

MTLD

NCME

NPV

OMS

OR

PCC
Geriatric Depression Scale (com 10 itens)

Geriatric Depression Scale (com 15 itens)

Geriatric Depression Scale (com 30 itens)

Geriatric Emotional Assessment of Pain

Grupo Focal

Global Fit Index (Índice de Ajuste)

Hospital Anxiety and Depression Scale

Hamilton Anxiety Rating Scale

Hamilton Depression Rating Scale

Health Related Quality of Life

Intervalo de Confiança

Índice de Concordância

Índice de Concordância de Conteúdo

Classificação Estatística Internacional de Doenças e Problemas

Relacionados à Saúde

International Classification Rating

Instituição de Longa Permanência para Idosos

Identificação de Resultados Não Intencionais.

Invariância

Índice de Validade de Conteúdo

Joanna Briggs Institute

Critério ou Teste de Kaiser Meyer Olkin

Liebowitz Social Anxiety Scale

Montgomery-Asberg Depression Rating Scale

Minimum Average Partial

Medical Subject Headings

Mini International Neuropsychyatric Interview

Teoria Motivacional de Vida e Desenvolvimento

Conselho Nacional de Medição em Educação

Negative Predictive Value

Organização Mundial de Saúde

Resultado de Análise Multivariada

População, Conceito, Contexto (critério mnemônico da Scoping Review) 


\begin{tabular}{|c|c|}
\hline PENSA & Processos de Envelhecimento Saudável (Estudo Longitudinal) \\
\hline PHQ-2 & Patient Health Questionnaire-2 \\
\hline PHQ-9 & Patient Health Questionnaire-9 \\
\hline $\mathrm{PICO}$ & $\begin{array}{l}\text { População, Intervenção, Grupo Comparador, Desfecho (Critério } \\
\text { Mnemônico para Revisão Sistemática) }\end{array}$ \\
\hline PPV & Positive Predictive Value \\
\hline PR & Preditivo \\
\hline PRÉ-T & Pré-teste \\
\hline PRISMA-ScR & $\begin{array}{l}\text { Preferred Reporting Items for Systematic reviews and Meta-Analyses } \\
\text { extension for Scoping Reviews }\end{array}$ \\
\hline PSI & Person Separation Index \\
\hline PSS & Perceived Stress Scale \\
\hline RASCH & Técnica da TRI \\
\hline RDWLS & Robust Diagonally Weighted Least Squares \\
\hline RMSEA & Root Mean Square Error of Approximation \\
\hline RMSR & Raiz do Resíduo Quadrado Médio \\
\hline RT & Técnica de Rotação \\
\hline ROC & Receiver Operating Characteristic \\
\hline SABE & Estudo Saúde, Bem-estar e Envelhecimento (estudo longitudinal) \\
\hline SAVI & Modelo de integração de Força e Vulnerabilidade \\
\hline SCAN & Schedule for Clinical Assesment in Neuropsychiatric \\
\hline SCID-CV & Entrevista Clínica Estruturada para Transtornos \\
\hline SDS & Zung Self-Rating Depression Scale \\
\hline SOC & Teoria de Seleção Otimização e Compensação \\
\hline SS & Sensibilidade e Especificidade \\
\hline SST & Teoria da Seletividade Socioemocional \\
\hline STAI & State-Trait Anxiety Inventory \\
\hline TAG & Transtorno de Ansiedade Generalizada \\
\hline TCT & Teoria Clássica dos Testes \\
\hline TESAUROS & Base de indexação \\
\hline TLI & Tucker-Lewis Index \\
\hline TR & Tempo de Resposta \\
\hline TRI & Teoria de Resposta ao Item \\
\hline
\end{tabular}


UPDRS

Unified Parkinson's Disease Rating Scale

WLSMV

Weighted Least / Squares Mean and Variance Adjusted 


\section{SUMÁRIO}

1 APRESENTAÇÃ

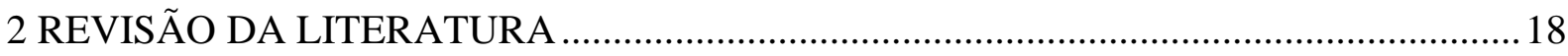

3 INSTRUMENTOS PSICOSSOCIAIS PARA IDOSOS BRASILEIROS: UMA SCOPING

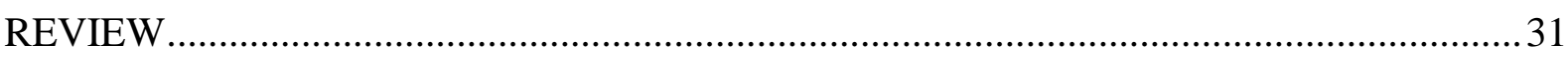

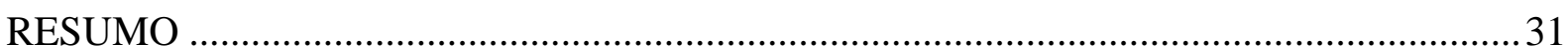

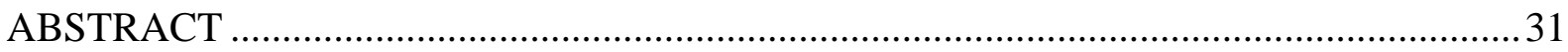

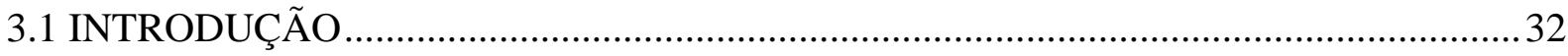

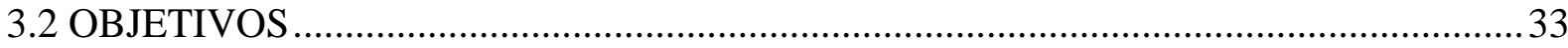

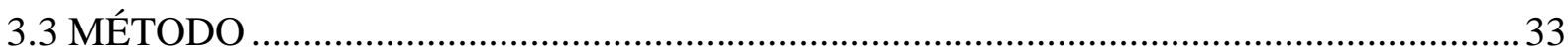

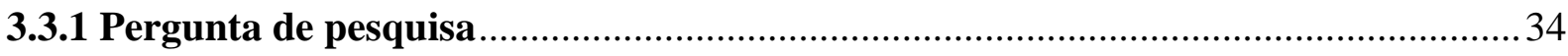

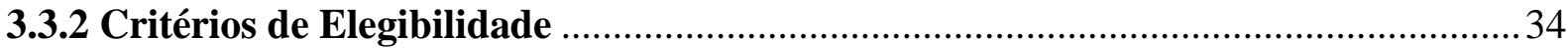

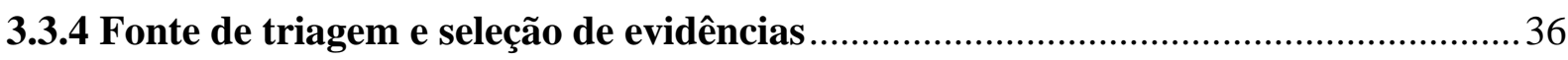

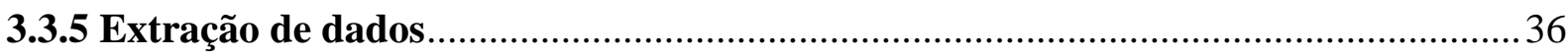

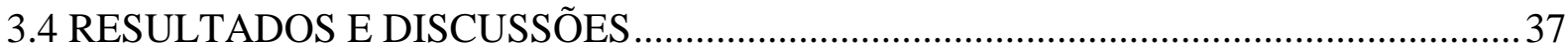

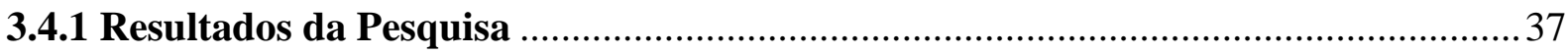

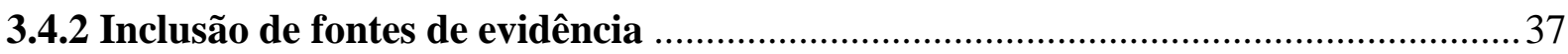

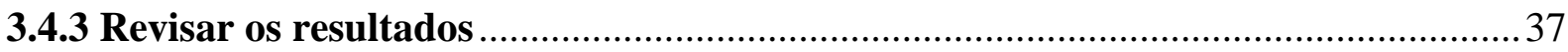

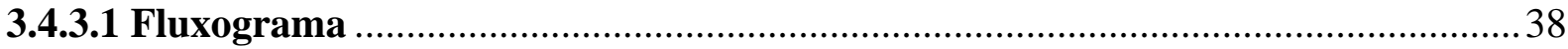

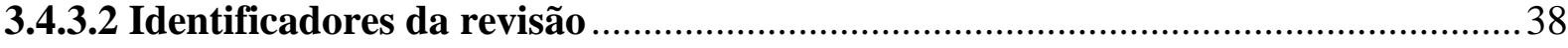

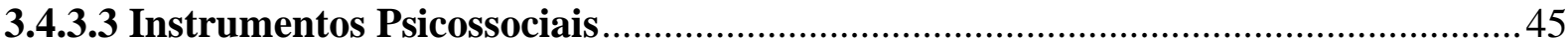

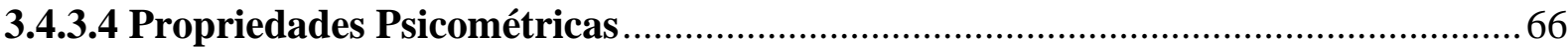

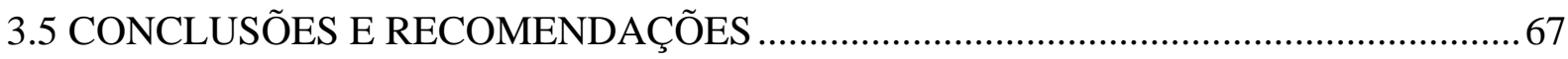

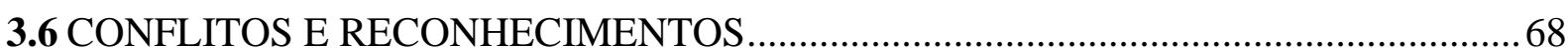

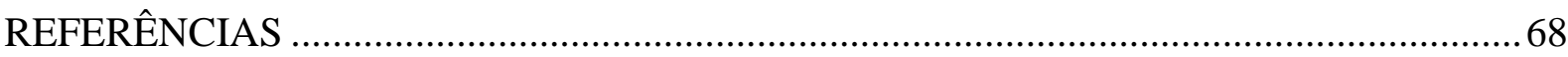


4 EVIDÊNCIAS DE VALIDADE DOS INSTRUMENTOS DE AVALIAÇÃO DE ANSIEDADE, DEPRESSÃO E ESTRESSE PARA IDOSOS BRASILEIROS: UMA

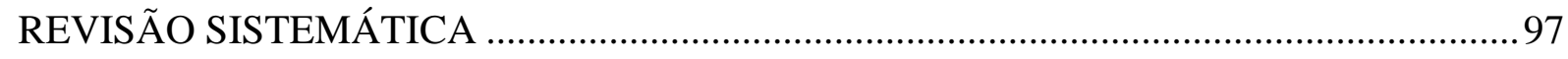

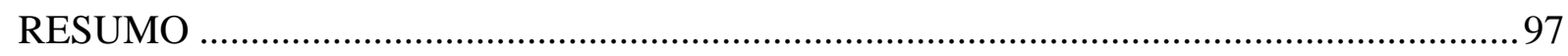

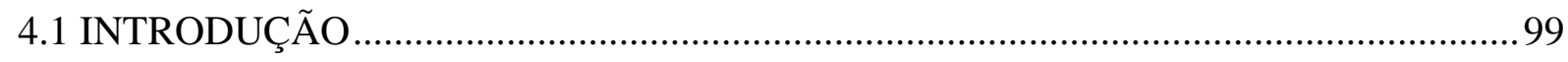

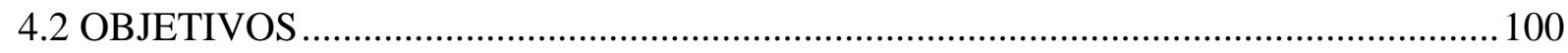

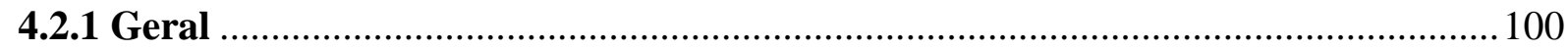

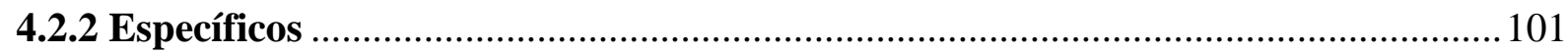

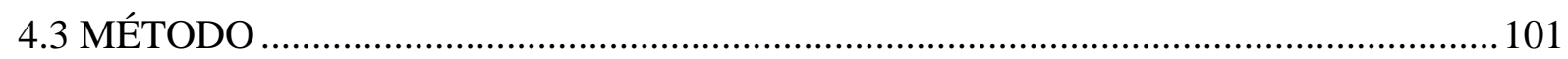

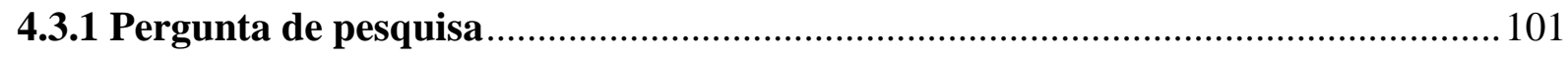

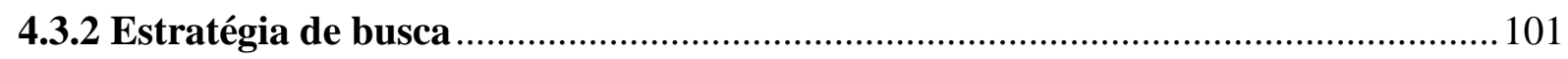

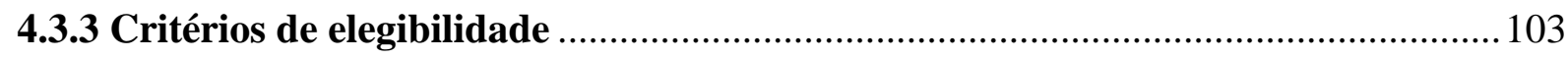

4.3.4 Seleção dos estudos, extração dos dados e análise da qualidade metodológica ..... 103

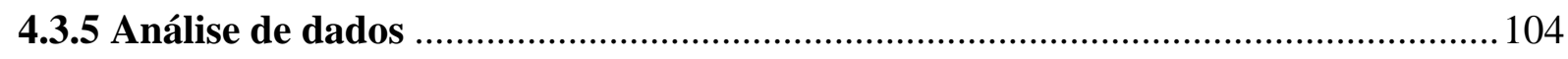

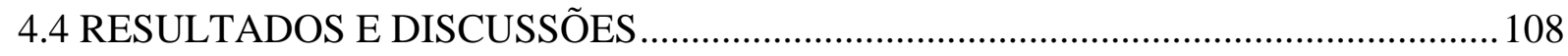

4.4.1 Etapa 1 - Síntese das evidências recuperadas, caracterização das amostras e dos

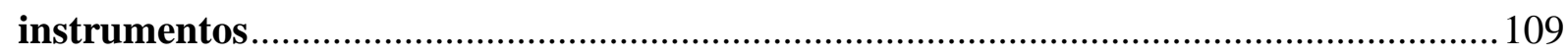

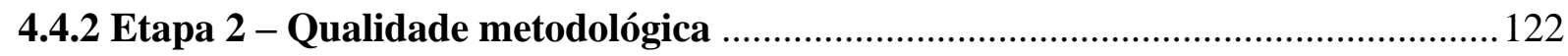

4.4.3 Etapa 3 - Evidências de validade .................................................................. 125

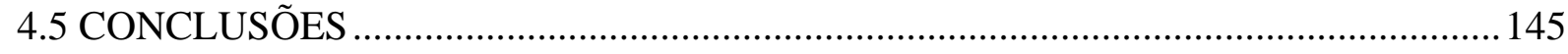

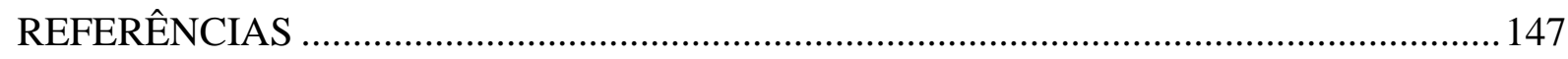

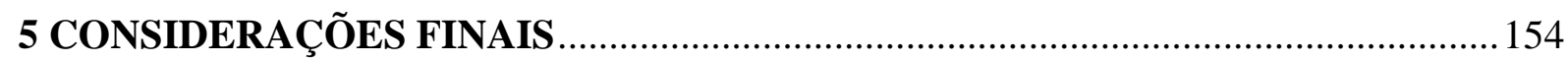

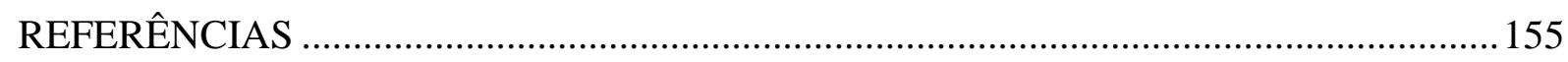




\section{APRESENTAÇÃO}

O processo de avaliação em idosos, em sua maioria, é relatado de forma segmentada na literatura científica, com foco na variável de interesse do pesquisador. Algumas iniciativas, buscam um olhar mais integrado, procurando atender aspectos biopsicossociais do indivíduo, como o caso da Avaliação Multidimensional do Idoso, também conhecida como Avaliação Geriátrica Ampla (AGA), que tem como objetivo o diagnóstico das condições de saúde, agudas e crônicas, ou seja, uma avaliação clínico-funcional (PARANÁ, 2018). A avaliação em idosos tem se mostrado como prática diversa nas diferentes áreas do conhecimento, principalmente nas áreas da saúde, com diferentes finalidades. Alguns instrumentos psicométricos são muito difundidos, mas com uso indiscriminado, sem justificativa da escolha ou evidências de validade apropriadas para a população específica. $\mathrm{O}$ olhar integrado para uma avaliação mais ampla pode inclusive favorecer resultados mais robustos e consistentes.

Instrumentos psicométricos fazem parte do processo de avaliação do idoso, exercendo grande influência nas tomadas de decisão, intervenções e criação de programas e políticas públicas para esta população. $\mathrm{O}$ interesse de pesquisadores e instituições nacionais e internacionais pela área é crescente, resultando em uma intensificação no desenvolvimento destes instrumentos (ALEXANDRE; COLUCI, 2011). Para que estes instrumentos de fato cumpram seu importante papel, é essencial que tenham sido desenvolvidos e validados rigorosamente como propõem a literatura científica, considerando principalmente as evidências de validade (ALEXANDRE; COLUCI, 2011; KESZEI; NOVAK; STREINER., 2010). O pesquisador/profissional precisa se apropriar das técnicas envolvidas para concluir cada etapa com êxito (COLUCI; ALEXANDRE; MILANI, 2015).

A dissertação apresentada desdobra-se em dois artigos sendo, uma scoping review e uma revisão sistemática das evidências de validade dos instrumentos. $\mathrm{O}$ primeiro artigo apresenta um mapeamento e categorização dos instrumentos de medida, com evidências disponíveis, que se propõem avaliar variáveis psicossociais em idosos brasileiros. O segundo artigo, apresenta uma revisão sistemática das propriedades de medida dos instrumentos identificados nos estudos recuperados que se propõem avaliar ansiedade, depressão e estresse. As evidências disponíveis recuperadas foram analisadas e apresentadas segundo as recomendações da literatura psicométrica contemporânea. 


\section{REVISÃO DA LITERATURA}

\subsection{Teorias sobre Envelhecimento}

O envelhecimento é um processo natural, progressivo e multifatorial, sendo resultado de diferentes aspectos: mudança biológica e comportamental, sua história no contexto social, lazer, religiosidade, vivências e adaptação a um novo estilo de vida, por isso, experimentado de forma heterogênea por cada ser humano. O envelhecimento tem sido observado sistematicamente e relatado sobre diferentes perspectivas, seja biológica ou filosoficamente. As diferentes lentes o relacionam com a sabedoria, respeito, poder, ou aspectos sociais como a aposentadoria, a moda, entre outros tantos temas que vão se integrando a vida humana de diferentes formas, da concepção à finitude (CAMPOS, 2011; CAMPOS; PÉCORA, 2015; FRIES; PEREIRA, 2011; PATHATH, 2017). Existem diversas teorias, a partir dos modelos supracitados, sendo que cada um deles, busca explicar o envelhecimento e seus paradigmas sob sua perspectiva.

Do ponto de vista biológico, destacam-se as teorias genéticas, as teorias dos danos e as teorias do desequilíbrio progressivo (CAMPISI, 2013; MARTINS, 2017), estas referem-se a um conjunto de mecanismos que atuariam isoladamente no processo de envelhecimento. Ran, Hou-Zao e De-Pei (2015) propõem um olhar conectando as teorias biológicas e discutem o envelhecimento em quatro camadas, sendo: disfunção imune metabólica e endócrina sistêmica, mau funcionamento celular e falha na manutenção de biomoléculas, buscando explicar o envelhecimento pelo viés biológico, como um processo integrado. Pathath (2017) retrata diferentes propostas sobre as causas do envelhecimento como, as teorias estocásticas, teorias da programação celular, teoria neuroendócrina, teoria da membrana do envelhecimento, teoria do limite Hayflick, teoria do declínio mitocondrial e teoria da ligação cruzada, onde diferentes perspectivas a níveis biológicos, químicos e fisiológicos são explorados, buscando as causas do envelhecimento. Entretanto, reconhece que mesmo neste âmbito, o envelhecimento é resultado de uma interação complexa ambiental e genética.

As teorias psicológicas do envelhecimento buscam explicar as mudanças de comportamentos associadas à idade, identificando aquelas que são típicas da velhice, daquelas que são compartilhadas por outras fases da vida, avaliando o equilíbrio entre as limitações e as potencialidades. Estes comportamentos de natureza psicológicas referem-se às emoções, personalidade, percepção, aprendizagem, memória, cognição, entre outros, que estão sujeitos a alterações mediante a idade cronológica, mas também à fatores patológicos, genéticos, 
ambientais e socioculturais (ALVES, 2017; SILVA, 2019). A perspectiva de Baltes (1987), chamada de lifespan, oferece uma lente em relação às mudanças e transições que ocorrem ao longo de toda a vida, aonde o sistema compensatório entre ganhos e perdas é afetado principalmente pelo contexto sociocultural. Já a perspectiva de Marshall (2009), nomeada life course, se preocupa em descrever os status que os indivíduos ocupam durante todo o envelhecimento na sociedade, pois acredita que as estruturas e instituições sociais moldam estes status, de acordo com cada época.

As teorias sociológicas do envelhecimento, se apresentam em pelo menos três ondas, partindo de 1961 com a teoria do desengajamento de Cumming e Henry (DOLL et al., 2007; SOUZA; SALES, 2016). Martins (2017) destaca a teoria da atividade que, relata que as pessoas se ajustam às mudanças de acordo com a idade, sendo que as pessoas mais ativas são as mais felizes, paralelamente, explica que os papeis perdidos por conta da idade são substituídos por novos papeis, assim o indivíduo mantem seu lugar em sociedade. Zacher e Rudolph (2017) dão um olhar diferente às teorias, reunindo em seu estudo sobre envelhecimento bem-sucedido no trabalho, uma revisão crítica sobre teorias que chamou de "teorias gerontológicas do envelhecimento bem-sucedido", agregando informações de mais de 10 teorias, percorrendo os anos de 1950 a 1980. Foram citadas as seguintes teorias: teoria da atividade (HAVIGHURST, 1961), teoria do desengajamento (CUMMING; HENRY, 1961), teoria da continuidade (ATCHLEY, 1971), modelo de Rowe e Kahn's (ROWE; KAHN, 1987), abordagem de recursos (NEUGARTEN, 1972), teoria de seleção, otimização e compensação-SOC (BALTES; BALTES, 1990), teoria motivacional de vida em desenvolvimento-MTLD (HECKHAUSEN; SHULZ, 1995), modelo de assimilação e acomodação por coping-AAC (BRANDSTADTER; RENNER, 1990), teoria da seletividade socioemocional-SST (CARSTENSEN, 1987; 1991), modelo de integração de força e vulnerabilidade-SAVI (CHARLES, 2010), critérios e estratégias para envelhecimento bem-sucedido no trabalho (ROBSON et al., 2006; ROBSON; HANSSON, 2007), definição de trabalho e ferramentas teóricas do envelhecimento bemsucedido no trabalho (ZACHER, 2015), o papel ativo dos empregados frente ao envelhecimento bem-sucedido no trabalho (KOOIJ; TIMS; KANFER, 2015). Este compilado relata também os mecanismos, princípios fundamentais e críticas de cada perspectiva. Apesar da lente para o trabalho, as teorias são independentes deste contexto, atravessam o tema, narrando uma linha do tempo da ascensão do envelhecimento pelo então olhar desenvolvimentista que atribuía a velhice apenas perdas e danos. Algumas teorias retratam o desenvolvimento psicossocial da velhice e envelhecimento com ênfase em um envelhecimento ativo (teorias da atividade, 
desengajamento e continuidade), outras foram marcadas por novos conceitos, como a diferenciação do envelhecimento normal e bem-sucedido (modelo de envelhecimento bemsucedido), outras ainda, fazem parte da perspectiva lifespan (abordagem de recursos, SOC, ACC, MTLD, SST e SAVI). Um estudo de Neri (2006), relatou a obra de Baltes (1939-2006), psicólogo alemão que tanto contribuiu para pesquisas do desenvolvimento e envelhecimento, reafirmando a importância do paradigma/perspectiva lifespan, que nas últimas décadas foi consolidada por pesquisas empíricas consideráveis, mediante as possibilidades da época, mas avança com novos pressupostos, correspondendo as necessidades atuais, implicando inclusive na perspectiva da psicologia do desenvolvimento. A partir de então, o envelhecimento extrapolou a visão do declínio e a partir da teoria SOC, oferece um modelo para pesquisas e intervenções em todo espectro do desenvolvimento/envelhecimento.

Para Alkema e Alley (2006), Ferraro (2006) e Palmore (1977, 1981) a gerontologia ainda precisa de teorias, métodos e instrumentos próprios, que fortaleçam o campo rumo à consolidação à uma disciplina. Sendo um grande desafio para a gerontologia, ressignificar o processo de envelhecimento, desvinculando preconceitos e combatendo o ageísmo. Compreender o novo perfil populacional, ofertar soluções multiprofissionais e prover um envelhecimento mais positivo e produtivo (SILVA, 2019). Em gerontologia, uma compreensão sólida da natureza do envelhecimento ainda está em construção, sendo que uma boa teoria, deve conceder maior coerência lógica para o campo/disciplina. Alguns autores afirmam que a gerontologia poderia ter se tornado rica em dados e pobre em teorias, muitas justificativas poderiam ser apresentadas para esta afirmação, entretanto, cabe uma reflexão de como a gerontologia está se desenvolvendo ou para onde está indo. Até agora, debateu e interpretou ideias advindas das ciências sociais, médicas, políticas e humanas, mas ainda precisa avançar em seu próprio campo. Discute-se que por ser a gerontologia um campo multidisciplinar, as outras disciplinas a coabitam, mas acabam não interagindo, ou seja, torna-se um espaço de pouco diálogo, o que levanta questões sobre limites e dominâncias. Frente a isso, teorizar na gerontologia requer a identificação dos aspectos que podem ser encarados como problema ou não, de forma dialógica, afetando tanto as práticas profissionais, como o cotidiano do idoso. Ressaltando que a teorização não é nunca a-política ou a-histórica (BIGGS; LOWENSTEIN; HENDRICKS, 2003).

2.2 Envelhecimento, velhice e idoso 
O processo de envelhecimento provoca então, alterações biológicas, psicológicas e sociais, como já referido, mas é na velhice que este processo se evidencia. O aparecimento de rugas e cabelos brancos, às alterações das funções orgânicas, são respostas das modificações biológicas, fisiológicas e químicas. Ao envelhecer, o ser humano precisa adaptar-se a cada situação nova do seu cotidiano (DARDENGO; MAFRA, 2018; DOS SANTOS; MOREIRA; CAERVENEY, 2016). A velhice está carregada de ideias negativas, geralmente percebida como uma fase com aspectos de limitações e perdas, repleta de estereótipos. A longevidade ainda é vista como um problema social, pois, necessita de apoio e estratégias políticas, sociais, econômicas e de saúde para atender esta demanda (SHI et al., 2016). No panorama de desenvolvimento humano, a velhice é desvalorizada e colocada à margem, evitada e rejeitada de diversas maneiras até pelo próprio idoso. Em contrapartida, as outras etapas da vida são contempladas e planejadas socialmente (LIMA; VIANA; LAZARINI, 2011; SANTOS, 2010). É importante destacar que a velhice é conceitualmente distinta do envelhecimento e pode ser compreendida como a última fase do processo de envelhecer, período da vida historicamente construído, contemplando valores culturais de uma dada sociedade. Historicamente observa-se que a velhice carrega uma marca social ligada à rejeição da figura do velho, em suma, alimentada pela ideia de improdutividade e declínio (LIMA; VIANA; LAZARINI, 2011; TEIXEIRA et al., 2016).

A velhice é uma etapa da vida com suas particularidades e características, assim como as outras, não necessariamente deve significar um tempo de sofrimentos, fragilidades e declínios, podendo também, ser vivida com qualidade, tendo o idoso como protagonista da própria vida (MESQUITA, 2016). Envelhecer com qualidade é uma possibilidade, mas por se tratar de uma longa e última etapa da vida, pode envolver sentimentos mistos de realização perante a vida que se passou e desespero em relação a vida que se finda. Definir velhice usando apenas a visão biológica é um equívoco de demarcação meramente cronológica, pois trata a população idosa de forma homogênea, não levando em consideração aspectos importantes do contexto sociocultural em que eles estão inseridos (LIMA; VIANA; LAZZARINI, 2011; LUIZ et al., 2018). O conceito legal de idoso ainda está atrelado a idade cronológica, ou seja, independente do seu estado biológico, psicológico e/ou social. Segundo a Organização Mundial da Saúde (OMS) em países em desenvolvimento, é considerado idoso pessoas a partir de 60 anos, já em países desenvolvidos o ponto de corte é de 65 anos. Entretanto, o conceito de envelhecimento é multidimensional e vasto, não devendo ser limitado a idade cronológica, neste sentido, surgem diferentes classificações para as faixas etárias do envelhecimento. Papália, 
Olds e Feldman (2006) distribuem as faixas etárias em três categorias, sendo: "idosos jovens" - entre 65 e 74 anos; "idosos velhos" entre 75 e 84 anos; e "idosos mais velhos" - acima dos 85 anos. Para Pathath (2017) a idade é uma invenção humana, um acordo social para demarcar os fatos em períodos cronológicos, que nada tem a ver com a capacidade física ou bem-estar psicológicos por exemplo. Universalmente, o parâmetro cronológico carrega classificações, buscando enquadrar os comportamentos humanos e estratificar o ônus e bônus de cada fase, ignorando muitas vezes todos os aspectos culturais envolvidos nessas classificações. A idade em si, não traz nenhuma explicação para os comportamentos humanos, visto que estes estão sempre em movimento. A idade cronológica, deve então ser compreendida, apenas como um marcador externo. Repensar o real significado de ser velho/idoso, é indispensável para a progressão da gerontologia/geriatria.

Se em 1970 a média de expectativa de vida era 61 anos, em 2018 a média da expectativa de vida era de 76 anos no Brasil (GAPMINDER, 2020), ou seja, viver a velhice superou a infância e a adolescência, provocando reflexões sobre o marco do seu início, tornando esta fase da vida cada vez mais relevante frente a compreensão da sua extensão, como alterações físicas, cognitivas e sociais. O aumento da longevidade, não somente no Brasil, traz como consequência o avanço de doenças crônicas e incapacidades funcionais. Apesar de serem mais intensas nessa fase, não estão sempre ligadas a dependências ou incapacidades, porém, podem gerar na maioria das vezes uma maior vulnerabilidade (ARSENIJEVIC et al., 2016; MIRANDA; MENDES; DA SILVA, 2016). Em contrapartida, como os indivíduos vivem mais, a qualidade de vida se torna um tema central para o bem-estar pessoal e social (VON HUMBOLDT; LEAL, 2014). Pesquisas longitudinais como: Estudo Longitudinal de Saúde dos Idosos (ELSI), Fragilidade em Idosos Brasileiros (FIBRA), Saúde, Bem-estar e Envelhecimento (SABE), EpiFloripa, são grandes contribuidores quando se trata de dados sobre condições de vida e saúde da população adulta e idosa. São retratadas as condições de saúde física e mental, bem-estar econômico, social e psicológico, examinando tendências de médio e longo prazo destas dimensões, em diferentes coortes, o que permite um amplo diagnóstico, elaboração e avaliação de políticas de saúde, serviços e ações orientadas às necessidades desta população. As características das coortes de idosos são muito diferentes entre si, por isso é importante que as pesquisas acompanhem as coortes ao longo dos anos, captando as variações das respostas aos padrões associados a essas mudanças (LEBRÃO et al., 2018).

A partir do EpiFloripa Idoso, foi avaliado recentemente, a associação entre a renda contextual e a incidência de incapacidade nas atividades básicas e instrumentais da vida diária 
de 1.196 idosos, após quatro anos, constatando que a incidência de incapacidade nas atividades básicas da vida diária foi de 15,8\% (IC 95\% 13,8-17,9) e nas atividades instrumentais da vida diária de 13,4\% (IC 95\% 11,6-15,5), com associação significativa entre a renda contextual e a incidência de incapacidade nas atividades básicas da vida diária, ou seja, renda contextual influencia no desenvolvimento de incapacidade nas atividades básicas da vida diária em idosos, por este motivo os autores recomendam ações para redução de iniquidades socioeconômicas e promoção da longevidade com independência (DANIELEWICZ; d'ORSI; BOING, 2019). Assim como, por meio do estudo FIBRA, foi avaliado, também recentemente, a prevalência e os fatores associados à fragilidade em Minas Gerais, com uma amostra de 461 indivíduos com 65 anos ou mais. A média de idade foi de 74,4 anos (DP $\pm 6,8$ ), 69,6\% eram mulheres e 71,9\% brancos. Foi constatado que a prevalência de fragilidade foi de 5,2\%; 49,9\% foram de indivíduos pré-frágeis. Idade avançada (OR: 6,4; IC 1,76-23,8), comprometimento das atividades básicas de vida diária (OR: 5,2; IC 1,1-23,1) e auto percepção de saúde ruim (OR: 0,13; IC 0,03-0,4), foram associados à fragilidade, ou seja, grande parte dos indivíduos apresentou-se frágil, enquanto que metade da amostra estava sob risco de progressão para esta condição, sugerindo que é urgente a adoção de medidas de saúde pública com objetivo de prevenção e redução de complicações (LOURENÇO et al., 2019).

\subsection{Heterogeneidade, complexidade e subjetividade na avaliação em idosos}

Para Lowsky (2014) a idade não parece ser um marcador muito adequado quando se fala em envelhecimento saudável, isso porque, seu estudo analisou cinco marcadores de bemestar (saúde auto-realatada, não receber ajuda ou supervisão nas AVDs ou AVDIs, avaliação sobre limitações para o trabalho - seja física ou mental, não ter diagnóstico de doenças crônicas - câncer, diabetes, doença cardíaca, doença pulmonar, AVC e ter pontuação adequada no instrumento HRQol) em indivíduos americanos. A partir do painel de despesas médicas e de saúde dos anos de 2003 e 2004, os indivíduos foram divididos em grupos nas seguintes faixaetárias: 51-54, 55-59, 60-64, 65-69, 70-74, 75-79, 80-84 e 85+, de acordo com os resultados, proporções significativas, em todas as idades, apresentaram de acordo com os indicadores descritos, uma vida saudável, desmistificando os declínios limitantes ligados à idade, despertando a necessidade de repensar estereótipos e expandir a compreensão de quais aspectos durante a trajetória de vida, podem ter impacto direto e/ou indireto para estas ocorrências positivas. Um outro estudo, também investigou a heterogeneidade entre idosos, porém, a partir da avaliação da cognição. Todos os 369 participantes, passaram por uma avaliação diagnóstica 
multidisciplinar, onde três domínios cognitivos foram avaliados (memória episódica, memória semântica e função executiva). As taxas individuais de mudanças mostraram-se heterogêneas em cada domínio, o que pode demonstrar limitações no diagnóstico transversal para prever prognóstico, ou seja, não há preditores robustos sobre mudanças cognitivas, desde a normalidade à demência (MUNGAS et al., 2010).

Estes achados refletem a importância de considerar as diferenças individuais e não generalizar está faixa etária, rotulando a presença ou ausência de patologias, a condição física, o desempenho cognitivo ou qualquer outra limitação ou capacidade, de forma tão estática. Se faz necessário o estabelecimento de padrões e classificações para melhor comunicação acadêmica-científica, entretanto, para a prática clínica, se manter vigilante a essas sutis ou drásticas diferenças são essenciais para uma melhor intervenção. A teoria dos sistemas ecológicos de Bronfenbrenner é uma perspectiva importante que pode apoiar este olhar mais ampliado para o idoso e o seu papel ativo frente à velhice. Baseia-se na ideia de que o desenvolvimento humano é fruto da interação entre o indivíduo e o meio ambiente, variando de acordo com o lugar e o tempo. A interdependência dos indivíduos e seus ambientes formam um sistema, que opera de maneiras favoráveis, ou desfavoráveis, ao desenvolvimento saudável, sendo o indivíduo um agente ativo, que contribui para o seu próprio ambiente (MRÓZEK, 2020; TUDGE; ROSA, 2019). O século XX foi deflagrado pelo envelhecimento acelerado e crescente da população global, que dramaticamente parece ter despertado a curiosidade ou um genuíno interesse dos pesquisadores, em compreender o que este fenômeno implicaria dali para frente. A tão sonhada longevidade, cada vez mais presente, se tornou motivo repentino de estudos e viralizou como justificativa de muitas pesquisas na literatura acadêmica, incluindo muitas vezes, o processo de avaliação.

A avaliação em idosos é complexa por uma série de fatores, como: a heterogeneidade da velhice, a complexidade e subjetividade de alguns construtos e apesar da vasta possibilidade de instrumentos, em sua maioria, estes não foram desenvolvidos/adaptados à todos os contextos socioculturais. Sendo assim, a avaliação requer do profissional: conhecimento, experiência e habilidades especificas (PASCHOAL, 2004). Diversos instrumentos, escalas de avaliação funcional e de avaliações específicas são utilizados amplamente por equipes interdisciplinares, por ser fundamental desde a triagem até a intervenção aos idosos. Uma avaliação eficiente e completa, a custos razoáveis, torna-se cada vez mais necessária. Objetivando o diagnóstico precoce de problemas de saúde e a orientação de serviços de apoio (MORAES et al., 2018; VERAS, 2019). 
Os instrumentos psicométricos são muito utilizados para decisões clínicas, pois muitos fenômenos não são diretamente observáveis, portanto, requerem instrumentos de medida que possibilitem sua detecção e/ou classificação, minimizando uma avaliação pobre ou equivocada. Estes instrumentos devem ser válidos e precisos para que consigam constatar o fenômeno como ele realmente é (CHAN, 2014; RAYCOV; MARCOULIDES, 2011). A escolha do instrumento adequado é essencial. Atualmente existem inúmeros instrumentos de medida, inclusive que se propõem a medir o mesmo construto, desenvolvidos para a mesma população. Pesquisadores e profissionais precisam estar atentos na escolha do instrumento mais adequado para sua aplicação, pois instrumentos com resultados inadequados introduzem vieses nas conclusões, descartando o projeto, desperdiçando recursos, ferindo a ética e prejudicando diretamente um indivíduo ou população (MOKKINK et al., 2016; SOUZA; ALEXANDRE; GUIRADELLO, 2017).

O primeiro passo para a seleção de um instrumento de medição é saber o que deverá ser medido, ou seja, a variável latente deve ser claramente definida. Ao medir uma variável ampla, por exemplo, deve ser esclarecido quais subdomínios são relevantes para a população-alvo no contexto específico de interesse. É preciso definir ou descrever explicitamente o conceito, caso não, interpretações distintas podem ocorrer, já que as vezes existem várias definições para uma mesma variável (MOKKINK et al., 2016). Em seguida, é preciso escolher um instrumento específico, para tanto, deve-se conhecer minimamente parâmetros de validade, que por métodos psicométricos adequados, avaliam a qualidade do instrumento de medida. Iniciativas como a Lista de verificação COSMIN, surgem para apoiar essa análise (CUNHA; ALMEIDA NETO; STACKFLETH, 2016; MOKKINK et al., 2016), mesmo com algumas limitações, principalmente dos conceitos contemporâneos de evidências de validade e de alguns indicadores ausentes.

As diferentes formas de aplicação dos instrumentos de medida (impresso, online, chamada telefônica), as características dos indivíduos (escolaridade, sexo, idade), o tempo de preenchimento do instrumento, o esforço necessário para a compreensão e preenchimento, tanto do avaliador quanto do avaliado, são importantes aspectos que impactam um instrumento e precisam ser planejados e preparados adequadamente. Estas e outras particularidades precisam ser analisadas em uma etapa de desenvolvimento ou para uma nova versão, resumida/adaptada, se for o caso (AROSON; FRIED; GOOD., 2002). Quando se trata de avaliações com idosos, é necessária uma compreensão dos processos fundamentais das mudanças comportamentais ao longo da vida, ou seja, ao longo do envelhecimento humano, principalmente devido aos 
estereótipos ligados à velhice. Compreender as patologias, mas também os aspectos típicos dessa fase, contribui para avaliações e ações mais ajustadas as necessidades individuais. Observar atentamente o que pode ser considerado como excepcional ou decorrentes do envelhecimento normal para esta faixa etária, são aspectos muito discutíveis, mas necessários, pois podem impactar diretamente na medição de uma variável latente (FERNANDES; SANTOS, 2015). Instrumentos desenvolvidos para avaliação da população geral permanecem os mesmos para a população idosa? Estes e outros questionamentos, devem ser feitos no planejamento das avaliações, seja em estudos ou na prática clínica. Poucos são os instrumentos desenvolvidos especialmente para esta população, especialmente para os idosos brasileiros.

Mediante ao cenário de necessidade da avaliação em idosos, é primordial que esses profissionais não apenas saibam aplicar e corrigir um teste/protocolo, mas também escolher os mais adequados de acordo com as evidências disponíveis para a população-alvo. Compreender basicamente o processo de desenvolvimento e validação, apoiam uma escolha mais consistente de instrumentos que resultam em processos mais responsáveis e assertivos (FRANÇA et al., 2016).

\subsection{Psicometria e suas contribuições}

Torna-se primordial o reconhecimento da importância da psicometria para a Gerontologia, pois ela se preocupa com o estudo dos problemas relacionados aos desafios da medição do comportamento humano, por meio de um conjunto específico de métodos desenvolvidos para gerenciá-los e/ou resolvê-los sistematicamente. Como disciplina científica, a psicometria baseia-se em abordagens, métodos e modelos matemáticos e estatísticos, válidos independentemente de qualquer teoria comportamental que um pesquisador possa estar adotando, sendo assim, fornece insumos essenciais para a pesquisa em ciências sociais e comportamentais, especialmente no que se refere ao desenvolvimento e seleção de procedimentos para a quantificação de observações nas variáveis de interesse estudadas durante o processo de envelhecimento (RAYCOV; MARCOULIDES, 2011).

Para mensurar aquilo que não é observável, principalmente, nas ciências sociais e da saúde, pesquisadores, profissionais e outros interessados, se utilizam dos instrumentos de medida, também chamados de instrumentos psicométricos. Esses instrumentos são desenvolvidos e/ou traduzidos, adaptados e validados para mensurar uma variável latente (CURADO; TELES; MARÔCO, 2014; OLIVEIRA; OLIVEIRA; NATAL, 2016). A variável latente, é pressuposta de modelos teóricos e estimadas em itens representativos de sua 
manifestação. Trata-se de uma fonte não observada da variabilidade sistemática em um conjunto de dados (BOLEN, 2002; BORSBOOM, 2008). Logo, definir claramente e contextualizar a origem do que está sendo proposto medir, é o primeiro passo para o desenvolvimento de um instrumento psicométrico.

Para Filho (2014) medição é um processo de comparação de algo determinado a um valor padrão. As medidas podem ser de dois tipos, a medida direta, aquela alcançada diretamente por um instrumento, como altura (estadiometro) e peso (balança), e a medida indireta, aquela que resulta da relação matemática que vincula a grandeza a ser medida com outras que sejam possíveis mensurar, ou seja, tipo de medida que representa uma variável latente. Essas podem ser medidas com instrumentos apropriados (escalas, questionários, testes), que buscam refletir comportamentos observáveis em seus itens. Um dos princípios básicos da física é que, nenhuma grandeza é medida com precisão absoluta, ou seja, as medidas são sempre aproximações (TOGINHO FILHO; ANDRELLO, 2010), entretanto, podemos analisar a acurácia do instrumento, conceito importante quando se fala de instrumento de medição, para compreender a proximidade da estimativa de seu parâmetro.

De um modo geral, os instrumentos são criados a partir de modelos teóricos, mas podem partir de modelos subjetivos, baseados na avaliação clínica. Devem ser considerados aspectos conceituais, como: definir o construto e a população; aspectos práticos, como: encargos para pacientes e avaliadores, e custos; o processo inclui várias etapas (ANDRADE; VALENTINI, 2018; REPPOLD; GURGEL; HUTZ, 2014). Inicialmente é importante definir qual a finalidade do instrumento, sendo uma importante decisão, se o instrumento deve avaliar se o indivíduo apresenta ou não determinada característica, ou então, se o instrumento deve retratar o nível/desempenho do indivíduo em relação a determinado construto. É preciso também estabelecer uma definição operacional para o que se pretende medir e isso inclui um conjunto específico de comportamentos, pois, teóricos diferentes, podem escolher comportamentos diferentes para avaliar o mesmo construto, lembrando que os itens precisam ser minimamente homogêneos. Em próximo estágio, é feito um piloto com uma pequena amostra de indivíduos e estes são convidados a opinarem sobre cada item, contribuindo para uma análise qualitativa e revisão dos itens. Posteriormente são realizados procedimentos estatísticos para análise dos itens, ocorrendo o reagrupamento de itens ou mesmo a exclusão de alguns. Estudos com busca de evidências de validade devem ser então conduzidos, a fim de se produzir versões mais bem ajustadas. É importante estabelecer guias de aplicação e interpretação do teste (COLUCI; ALEXANDRE; MILANI, 2015; RAYCOV; MARCOULIDES, 2011). 
O estudo das propriedades psicométricas dos testes, em grande parte se baseiam na Teoria Clássica dos Testes (TCT), aonde dois principais postulados ditam primeiramente que, o resultado observado do teste é resultante da soma do resultado verdadeiro do indivíduo, com um erro associado a este resultado e também, que o valor esperado para o resultado observado é o resultado verdadeiro, assumindo que os erros aleatórios da medida, devem ser independentes em todas as circunstâncias. Na TCT, os itens são escolhidos por sua "qualidade", que são avaliados a partir da sua dificuldade (busca-se de 0 a 1 quantos indivíduos não responderam/responderam afirmativamente o item) e sua discriminação (busca diferenciar grupos que tiveram baixa ou alta pontuação). Já a Teoria de Resposta ao Item (TRI), surge após os anos 50, buscando superar as limitações discutidas da TCT, sem confrontá-la. Sua análise é centrada nos itens, pressupondo que existe no indivíduo um traço latente relativo a uma característica do indivíduo. O nível do traço latente, permite estimar a reposta positiva ou negativa à cada item, estabelecendo uma probabilidade da resposta relacionada a presença/intensidade da condição avaliada (SARTES; SOUZA-FORMIGONI, 2013; COUTO; PRIMI, 2011). Cada uma das teorias, parte de um pressuposto diferente e exige do pesquisador conhecimentos distintos para utilização das técnicas e interpretabilidade dos resultados.

A lógica de validade atravessou distintos conceitos na literatura, importantes de serem discutidos e compreendidos, pois é referida como elementar no debate psicométrico. Desde 1950 a Associação Americana de Psicologia (APA), a Associação Americana de Pesquisa Educacional (AERA) e o Conselho Nacional de Medição em Educação (NCME) colaboram com o desenvolvimento de padrões para testes educacionais e psicológicos, com as sucessivas edições dos Standards, proporcionando o conhecimento e uso sólido e ético dos testes, bem como práticas de avaliação da qualidade destes. Cada edição trouxe à luz novos consensos sobre validade, que foi evoluindo ao longo dos debates da área, padrões reconhecidos internacionalmente (NEWTON; SHAW, 2013).

Tradicionalmente, na primeira edição do Standards, a validade era definida e explicada segundo 19 padrões de qualidade e foram propostos quatro tipos de validade: conteúdo (determinar como o indivíduo se apresentaria no momento de um determinado universo de situações), preditiva (prever o desempenho futuro do indivíduo em uma variável externa), concorrente (estimar o status atual do indivíduo em uma variável externa) e construto (indeferir até que ponto um indivíduo possui uma característica) (AERA; APA; NCME, 1954).

Mais tarde, validade preditiva e concorrente se tornaram validade de critério e a visão tripartida foi altamente difundida. Na terceira edição do Standards, o conceito de validade ficou 
prejudicado, pois apresentava tanto "tipos" quanto "aspectos", o que não tratava o tema com clareza. Desde a quarta edição, validade reflete uma visão unificada e tratada como evidências de validade e não mais tipos de validade (AERA; APA; NCME, 1985).

A partir de uma reformulação proposta por Messick (1995), a quinta edição do Standards (1999) complementou a quarta com a proposta das cinco fontes de evidências de validade (AERA; APA; NCME, 1999). Mais recentemente, segundo o Standard, validade é o grau em que evidência e teoria apoiam as interpretações dos resultados dos testes em relação ao uso proposto (AERA; APA; NCME, 2014). Por meio de diversas discussões de estudiosos da área, Bandalos (2018) retrata validade não mais como uma visão tripartida, mas sim como evidências integradas em uma única avaliação geral, em relação ao significado das pontuações dos testes. Rompendo uma visão segmentada do processo de validação, que não forneceria evidências suficientes, separadamente, para atribuir validade ao teste. Por este motivo, a visão unificada de validade é amplamente aceita, cabendo ao usuário do teste, avaliar as evidências disponíveis e analisar em que grau sua interpretação é apropriada. De um lado, teóricos afirmam que não é o teste em si que é validado, mas sim, as inferências feitas a partir dos resultados dos testes, por outro, alguns ainda afirmam que validade está ligada diretamente ao teste. Para a autora, esta relação é inerente, aonde validade trata-se do teste, da pontuação do teste, interpretação e uso do teste. Sendo assim, AERA, APA e NCME (2014) enfatiza que a validação é um processo e não um estudo com visão dicotômica definindo se o teste está ou não está validado.

Para Messick (1995) todos os tipos de validade, se referiam ao construto e validade de um instrumento está ligada a um conjunto de evidências científicas, a aplicabilidade para o contexto específico e a interpretabilidade proposta. As cinco fontes de evidências de validade, descritas no último Standard (AERA; APA; NCME, 2014), evidências de validade com base no conteúdo (está relacionado com a construção dos itens, mensuração adequada do fenômeno), com base no processo de resposta (relação entre os processos mentais ligados aos construtos e as respostas aos itens), com base na estrutura interna (trata-se da legitimação da representatividade dos traços latentes, concordando com a teoria psicométrica defendida), com base na relação com outras variáveis (quando o escore do teste se relaciona com outras medidas padrão) e com base na consequência da testagem (relaciona-se com o uso do escore do teste, com a responsabilização da medida e suas consequências) foram reconhecidas e amplamente aceitas como atualização do conceito (BANDALOS, 2018; FURR, 2018; HAIR et al., 2014), para tanto é importante compreender os procedimentos e técnicas estatísticas que traduzem e possibilitam o alcance de cada fonte de evidência. Para conteúdo do teste: deve-se verificar a 
ocorrência de painel com especialistas, garantir que os traços latentes adequados estejam representados nos itens, que eles sejam relevantes, de acordo com a realidade-cultural, abrangente, compreensível, que se apresente de forma adequada, que seja utilizado um protocolo, analisada a concordância de conteúdo; para processos de resposta: deve-se verificar a testagem na população-alvo, pré-teste, garantia da compreensão dos itens, acompanhamento do tempo de resposta; para estrutura interna: apresentação do cálculo amostral, matriz de correlação, verificação da dimensionalidade, técnica de extração, técnica de rotação, técnicas da TRI, análise da consistência interna, análise da invariância, cálculo do escore; em relação com outras variáveis: padrão de referência para o mesmo construto, para construtos diferentes ou opostos, apresentação de sensibilidade e especificidade, comparação das diferenças entre grupos; e para consequência da testagem: relatos de benefícios previstos e identificação de resultados não intencionais (BANDALOS, 2018; FURR, 2018).

Apesar das recomendações do Standards referente ao desenvolvimento e avaliação de testes e práticas de testagem, muitos instrumentos são disponibilizados e inclusive comercializados indevidamente, sem manual, recomendações ou evidências de validações essenciais (CHAN, 2014). Os instrumentos com poucas evidências de validade podem impactar negativamente a prática clínica ou para qual seja sua finalidade, já que sua medida resultará em uma decisão com o outro, seja diretamente por meio de uma intervenção ou indiretamente como a criação de políticas públicas. Diante do apresentado, esta Dissertação se dedicou a um mapeamento sistematizado dos instrumentos psicossocias disponíveis, para o uso em avaliações com idosos brasileiros, bem como uma análise das evidências de validade destes instrumentos. Esse tipo de investigação é importante para que as orientações sobre os instrumentos sejam claras, já que o desenvolvimento e uso de instrumentos é cada vez mais frequente. É importante ressaltar que o primeiro estudo, refere-se a uma tentativa de recuperação do máximo de evidências possíveis, reunindo em uma única fonte, um macro-olhar sobre os instrumentos disponíveis na literatura. Já o segundo estudo, tem a proposta de uma análise macro-micro dos processos de validação, considerando as técnicas empregadas pelos estudiosos, visto que há uma série de problemas nos procedimentos adotados na atualidade (NEWTON, 2016). Cada um dos estudos foi apresentado segundo as normas e recomendações do Programa de Pós-Graduação em Gerontologia da Escola de Artes, Ciências e Humanidades de São Paulo. 


\section{INSTRUMENTOS PSICOSSOCIAIS PARA IDOSOS BRASILEIROS: UMA SCOPING REVIEW}

\section{RESUMO}

Este estudo objetivou mapear e categorizar os instrumentos de avaliação psicossocial, com evidências psicométricas disponíveis, para idosos brasileiros. Por meio de uma scoping review, foram identificados 5.258 estudos e após aplicação dos critérios de elegibilidade, 237 foram incluídos para síntese analítica. Parte dos dados extraídos foram apresentados em quatro blocos: Identificadores da revisão (autoria, tipo e ano de publicação, ligação com outros estudos/projetos, periódico e idioma); População (país, Estado, cidade, local de recrutamento, características da população, quantidade e idade); Instrumentos Psicossociais (nome em inglês/origem, acrônimo, variável latente); Propriedades psicométricas (tipo de evidência analisada/publicada). Destacaram-se 192 instrumentos que foram organizados em cinco subcategorias sendo, "Ansiedade, Depressão e Estresse", "Atividade Física, Capacidade Funciona e Mobilidade", "Inteligência e Cognição", "Saúde, Bem-estar e Qualidade de Vida" e "Síndromes Geriátricas e Violência". Este estudo não esgota as alternativas de instrumentos disponíveis para esta população, mas possibilita uma visão mais ampliada, reunindo em uma única fonte, evidências que possam gerar reflexão e agregar para a pesquisa e a prática de todos os interessados pela temática. A Gerontologia é um campo multidisciplinar crescente e necessita de especialistas para responder os diversos dilemas que surgem todos os dias frente a ascensão cronológica da vida humana e o mundo moderno.

\section{ABSTRACT}

This study aimed to map and categorize the psychosocial assessment instruments, with available psychometric evidence, for elderly Brazilians. Through a scoping review, 5.258 studies were identified and after applying the eligibility criteria, 237 were included for analytical synthesis. Part of the extracted data was presented in four blocks: Review identifiers (authorship, type and year of publication, link to other studies/projects, journal and language); Population (country, state, city, place of recruitment, population characteristics, quantity and age); Psychosocial Instruments (name in English/origin, acronym, latent variable); Psychometric properties (type of evidence analyzed / published). 192 instruments stood out, which were organized into five subcategories, being "Anxiety, Depression and Stress", "Physical Activity, Ability to Work and Mobility", "Intelligence and Cognition", "Health, 
Well-Being and Quality of Life" and "Geriatric's Syndromes and Violence”. This study does not exhaust the alternatives of instruments available to this population, but allows a broader view, gathering in a single source, evidence that can generate reflection and add to the research and practice of all those interested in the theme. Gerontology is a growing multidisciplinary field and needs specialists to answer the various dilemmas that arise every day in the face of the chronological rise of human life and the modern world.

\subsection{INTRODUÇÃO}

O envelhecimento é um processo constante e dinâmico, deflagrado por aspectos biológicos, psíquicos e sociais, que influenciam diretamente em como uma variável latente será manifestada em cada fase da vida. Na velhice, considerada última fase deste processo, as doenças crônicas são mais frequentes, o que não representa sinônimo de incapacidade e/ou dependência, mas sim, de maior vulnerabilidade (VERAS, 2019; PARANÁ, 2018). Logo, obter diagnósticos precoces e rápidos a custos razoáveis, são essenciais, principalmente para o sistema de saúde pública.

Nesse cenário, instrumentos de medida fazem parte da prática clínica, da avaliação em saúde e de pesquisas, sendo úteis e capazes de apresentar resultados robustos quando desenvolvidos e validados adequadamente (MEDEIROS et al., 2016). Os instrumentos devem ser desenvolvidos e/ou adaptados e validados para cada população específica, visto a necessidade de compreensão das características de cada uma delas, que podem afetar a manifestação da variável de interesse (PEDREIRA, 2016). As variáveis psicossociais ganham destaque para o idoso, uma vez que a velhice é uma construção social, experienciada de forma heterogênea e marcada por perdas e ganhos, que se sobrepõem aos marcadores biológicos. Assim, os métodos e as técnicas de investigação devem garantir indicadores seguros e confiáveis para esta população, respeitando suas características e manifestações distintas para cada constructo.

O acervo brasileiro de instrumentos desenvolvidos, adaptados culturalmente e validados para esta população é crescente, mas ainda muito questionável (VERAS, 2019; PARANÁ, 2018; APOSTOLO, 2012). A reprodução de estudos sem questionamento da escolha do instrumento, a replicabilidade do uso na prática clínica ou indicações informais para determinar o uso de um instrumento, reflete o uso indiscriminado, gerando diagnósticos distorcidos, tomadas de decisões errôneas entre outros danos que podem ser irreversíveis. Esse apontamento não se limita ao Brasil, de acordo com (KUANG et al., 2018) há um número reduzido de 
instrumentos desenvolvidos especificamente para a população idosa. Usualmente o instrumento original foi aplicado à outras populações e são posteriormente replicados na população idosa sem evidências suficientes de que ele mantém a propriedade psicométrica. Os autores complementam, afirmando que idosos podem ter um bom desempenho no funcionamento diário, embora possam pontuar baixo em testes padronizados e avançam apontando que imagens estereotípicas da velhice podem afetar a medição em todas as fases do processo de avaliação psicológica, incluindo a definição e seleção de medidas relevantes (por exemplo, saúde, desempenho de memória), o desenvolvimento e escolha de itens relevantes ou tarefas de desempenho, bem como o procedimento, aplicação e interpretação dos resultados individuais.

Baltes e Carstensen (1996) destacam que na velhice, as diferenças individuais e mudanças interindividuais são bastante comuns, e muitas vezes são mais pronunciadas do que nas fases anteriores da idade adulta. Portanto, torna-se essencial a realização desta scoping review, para um mapeamento sistematizado dos instrumentos de avaliação psicossocial disponíveis para idosos brasileiros, bem como propor a reflexão da escolha para a utilização, mediante as evidências científicas disponíveis na literatura.

\subsection{OBJETIVOS}

\subsubsection{Geral}

Mapear os instrumentos de medida psicossocial, com evidências psicométricas disponíveis, utilizados para a avaliação de idosos brasileiros.

\subsubsection{Específicos}

Caracterizar os estudos e população-alvo envolvida; identificar os instrumentos de medida e categorizar de acordo com a variável latente; e descrever os tipos de evidências consideradas em cada estudo.

\subsection{MÉTODO}

Por meio de uma revisão de escopo (scoping review) foram examinados a extensão, o alcance e a natureza das evidências dos instrumentos de avaliação de medida psicossocial disponíveis para idosos brasileiros. Esta revisão foi conduzida de acordo com as recomendações JBI Manual for Evidence Synthesis (Peters et al., 2020) e Preferred Reporting Items for Systematic reviews and Meta-Analyses extension for Scoping Reviews (PRISMA-ScR) Checklist 
(Tricco et al., 2018). Primeiramente, foi realizada uma investigação referente as evidências de validade de estudos originais (desenvolvimento inicial) e cross-culturais (tradução e/ou adaptação). Após localização dos estudos, o principal objetivo foi a categorização destes instrumentos, sendo organizados em subgrupos de acordo com a variável latente a que se propõem avaliar e por fim, outras características consideradas mais relevantes foram descritas.

\subsubsection{Pergunta de pesquisa}

Atendendo ao critério mnemônico PCC (P - população: idosos ( $\geq 60$ anos); C conceito: instrumentos de avaliação psicossocial; e C - contexto: qualquer estudo com evidência psicométrica (tradução e adaptação transcultural, desenvolvimento, validade, confiabilidade, etc), elaborou-se a questão de pesquisa ("Quais são os instrumentos de medida, com evidências psicométricas disponíveis, para a avaliação de variáveis psicossociais em idosos brasileiros?") que norteou este estudo.

\subsubsection{Critérios de Elegibilidade}

A amostra dos estudos incluídos contemplou, exclusiva ou parcialmente, idosos com $\geq 60$ anos; visto a definição desta população no Brasil, oficialmente, por meio deste marco cronológico. Além disso, foram contemplados apenas instrumentos que se propuseram a mensurar variáveis psicossociais, originais (desenvolvimento inicial) e cross-culturais (tradução e/ou adaptação), por serem fontes de evidências psicométricas do instrumento referido para a população especificada. Foram considerados ainda, artigos, dissertações ou teses; publicados em português, inglês e espanhol, disponíveis na íntegra, até 30 de setembro de 2020 .

\subsubsection{Estratégia de pesquisa}

As bases de dados eletrônicas utilizadas para a busca dos estudos foram: PubMed, Biblioteca Virtual da Saúde (BVS), Embase, Scielo, PsyArticles, Cinahl e Ageline, estas foram escolhidas visto sua relevância e por abarcar de forma abrangente a literatura sobre a temática. Inicialmente foi realizada uma busca em duas bases de dados (PubMed e BVS), a fim de uma análise das palavras contidas nos títulos e resumos dos artigos recuperados. Posteriormente, a estratégia foi testada em todas as bases consideradas para este estudo e por fim, as estratégias foram desenvolvidas a partir das seguintes considerações: vocabulário de indexação de cada base de dados (MeSH; DeCS; EMTREE; TESAUROS;), palavras-chaves relevantes na 
literatura gerontológica e cruzamento de acordo com a especificidade de cada base de dados, que podem ser conferidos na Tabela 1. Todas as buscas foram realizadas com o recurso de "busca avançada" e a utilização dos operadores booleanos, com a finalidade de refinar e identificar estudos mais alinhados com o objetivo deste estudo. Sendo que a busca final foi realizada em 01 de outubro de 2020.

Tabela 1. Estratégia de busca de acordo com cada base referenciada, 2020

\begin{tabular}{|c|c|}
\hline Base de dados & Estratégia de busca utilizada \\
\hline PubMed & $\begin{array}{l}\text { (((Aged OR Aging OR Elderly OR Older people) AND (Geriatric assessment OR mental } \\
\text { status and dementia tests OR psychiatric status rating scales OR evaluation studies as topic } \\
\text { OR assessment OR instrument)) AND (Validation study OR validation studies as topic OR } \\
\text { psychometrics OR validation studies)) AND (brazil) }\end{array}$ \\
\hline BVS & (tw:(aged)) AND (tw:(validation study)) AND (tw:(brazil)) \\
\hline Scielo & (aged) AND (validation study) AND (brazil) \\
\hline Embase & $\begin{array}{l}\text { ('aged'/exp OR aged OR 'aging'/exp OR aging OR 'elderly'/exp OR elderly OR 'older } \\
\text { people'/exp OR 'older people' OR (older AND people)) AND (('geriatric assessment'/exp } \\
\text { OR 'geriatric assessment' OR (('geriatric'/exp OR geriatric) AND ('assessment'/exp OR } \\
\text { assessment)) OR 'mental status'/exp OR 'mental status' OR (mental AND status)) AND } \\
\text { ('dementia tests' OR (('dementia'/exp OR dementia) AND tests)) OR 'psychiatric status } \\
\text { rating scales'/exp OR 'psychiatric status rating scales' OR (psychiatric AND status AND } \\
\text { rating AND scales) OR 'evaluation studies as topic'/exp OR 'evaluation studies as topic' OR } \\
\text { (('evaluation'/exp OR evaluation) AND ('studies'/exp OR studies) AND as AND topic) OR } \\
\text { 'assessment'/exp OR assessment OR 'instrument'/exp OR instrument) AND ('validation } \\
\text { study'/exp OR 'validation study' OR (('validation'/exp OR validation) AND ('study'/exp OR } \\
\text { study)) OR 'validation studies as topic'/exp OR 'validation studies as topic' OR } \\
\text { (('validation'/exp OR validation) AND ('studies'/exp OR studies) AND as AND topic) OR } \\
\text { 'psychometrics'/exp OR psychometrics OR 'validation studies'/exp OR 'validation studies' } \\
\text { OR (('validation'/exp OR validation) AND ('studies'/exp OR studies))) AND ('brazil'/ }\end{array}$ \\
\hline PsyArticles & 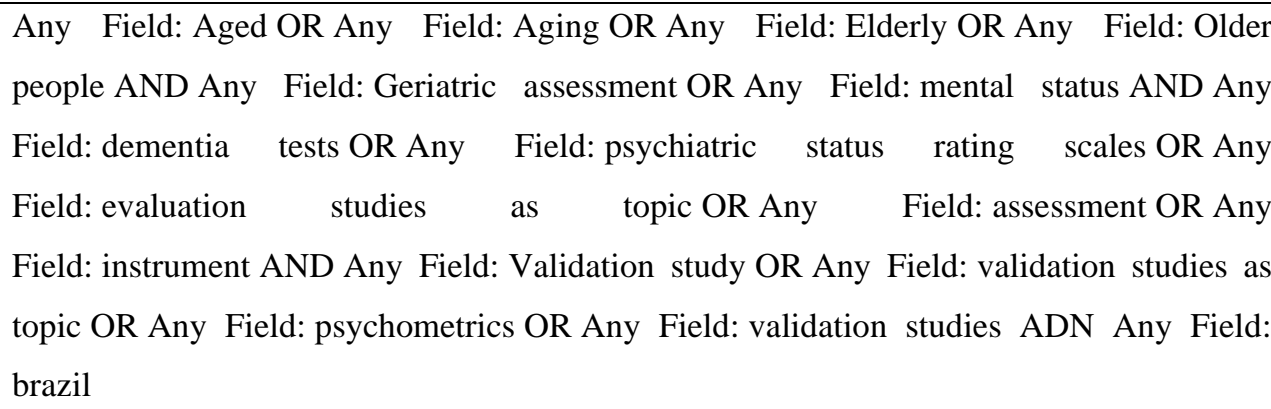 \\
\hline Cinahl & $\begin{array}{l}\text { (((Aged OR Aging OR Elderly OR Older people) AND (Geriatric assessment OR mental } \\
\text { status and dementia tests OR psychiatric status rating scales OR evaluation studies as topic }\end{array}$ \\
\hline
\end{tabular}




\begin{tabular}{ll}
\hline OR assessment OR instrument)) AND (Validation study OR validation studies as topic OR \\
psychometrics OR validation studies)) AND (brazil) \\
\hline (((Aged OR Aging OR Elderly OR Older people) AND (Geriatric assessment OR mental \\
status and dementia tests OR psychiatric status rating scales OR evaluation studies as topic \\
OR assessment OR instrument)) AND (Validation study OR validation studies as topic OR \\
psychometrics OR validation studies)) AND (brazil) \\
\hline
\end{tabular}

Fonte: Aline Cristina da Silva, 2020

\subsubsection{Fonte de triagem e seleção de evidências}

Para esta etapa utilizou-se o PRISMA 2009 Flow Diagram (Preferred Reporting Items for Systematic Reviews and Meta-Analyses), aonde dois revisores independentes conduziram a triagem e seleção dos estudos, Figura 1. Para os casos de divergência, em cada etapa, ambos discutiram caso a caso, até concordância de inclusão ou exclusão do estudo. Os estudos foram buscados de acordo com as estratégias definidas em cada uma das bases de dados descritas acima.

Nesta primeira etapa, foi realizada a exclusão de todos os estudos duplicados, posteriormente, foi realizada a leitura de todos os títulos e resumos para definição de inclusão e/ou exclusão para a próxima etapa. Em seguida, foi realizada a leitura na íntegra de todos os artigos para análise dos critérios de elegibilidade. Por fim, os artigos que atenderam os critérios, foram incluídos para a síntese analítica.

Para as etapas descritas acima, foi utilizado o software gratuito e online Rayyan QCRI, designado à estudos de Revisões Sistemáticas e suas variações, aonde os arquivos de dados de cada base, foram "upados", organizados e classificados de acordo com as etapas deste estudo.

\subsubsection{Extração de dados}

Os dados de interesse foram extraídos e registrados em uma tabela do Microsoft ${ }^{\circledR}$ Excel $^{\circledR}$ para Office 365 MSO. Esta tabela foi desenvolvida pela autora, baseada no Appendix 11.1 JBI template source of evidence details, characteristics and results extraction instrument (2020) e Cochrane Consumers and Communication Group Data extraction template for included studies (2016). Parte dos dados extraídos serão apresentados neste estudo de acordo com os seguintes blocos: Identificadores da revisão (autoria, tipo e ano de publicação, ligação com outros estudos/projetos, periódico e idioma); População (país, Estado, cidade, local de recrutamento, características da população, quantidade e idade); Instrumentos Psicossociais (nome em 
inglês/origem, acrônimo, variável latente); Propriedades psicométricas (tipo de evidência analisada/publicada).

Uma revisão de escopo examina questões amplas de pesquisa e, portanto, este estudo não reportará análise de qualidade metodológica, bem como não discutirá qualitativamente as escolhas dos autores em relação aos objetivos, métodos, resultados e conclusões obtidas. Se ocupará em organizar de forma sistematizada, os instrumentos com evidências psicométricas disponíveis para a população em questão, descrevendo as informações mais relevantes.

\subsection{RESULTADOS E DISCUSSÕES}

\subsubsection{Resultados da Pesquisa}

De acordo com a busca estabelecida, foram identificados 5.258 estudos (Figura 1) e removidos 2.044 estudos duplicados. Logo, foram selecionados 3.214 estudos para a leitura de “Títulos e Resumos", aonde 2.934 estudos foram excluídos. Posteriormente, 280 estudos foram selecionados, lidos na íntegra e avaliados segundo os critérios de elegibilidade.

Todas estas etapas foram realizadas por dois pesquisadores independentes, que concordaram que 43 estudos fossem excluídos, sendo: 24 por não contemplarem população idosa em sua amostra, nove por serem apenas resumo, cinco por serem realizados com população de outros países que não o Brasil, três por serem carta ao editor, um por não informar a localidade de coleta de dados/nacionalidade da amostra e um por não estar disponível na integra e mesmo após contato com o autor, não foi possível recuperá-lo, o que resultou na inclusão de 237 estudos para a síntese analítica.

\subsubsection{Inclusão de fontes de evidência}

Para esta scoping review foram incluídos apenas estudos completos publicados (artigos, dissertações ou teses) nos idiomas inglês, espanhol ou português, que contemplassem em sua amostra idosos brasileiros. Estes estudos, referem-se a algum tipo de evidência de validade do instrumento de medida referido, que se propôs a medir qualquer variável psicossocial, também, em idosos brasileiros.

\subsubsection{Revisar os resultados}




\subsubsection{Fluxograma}

Figura 1. Fluxograma do processo de revisão de escopo adaptado da declaração PRISMA, 2020
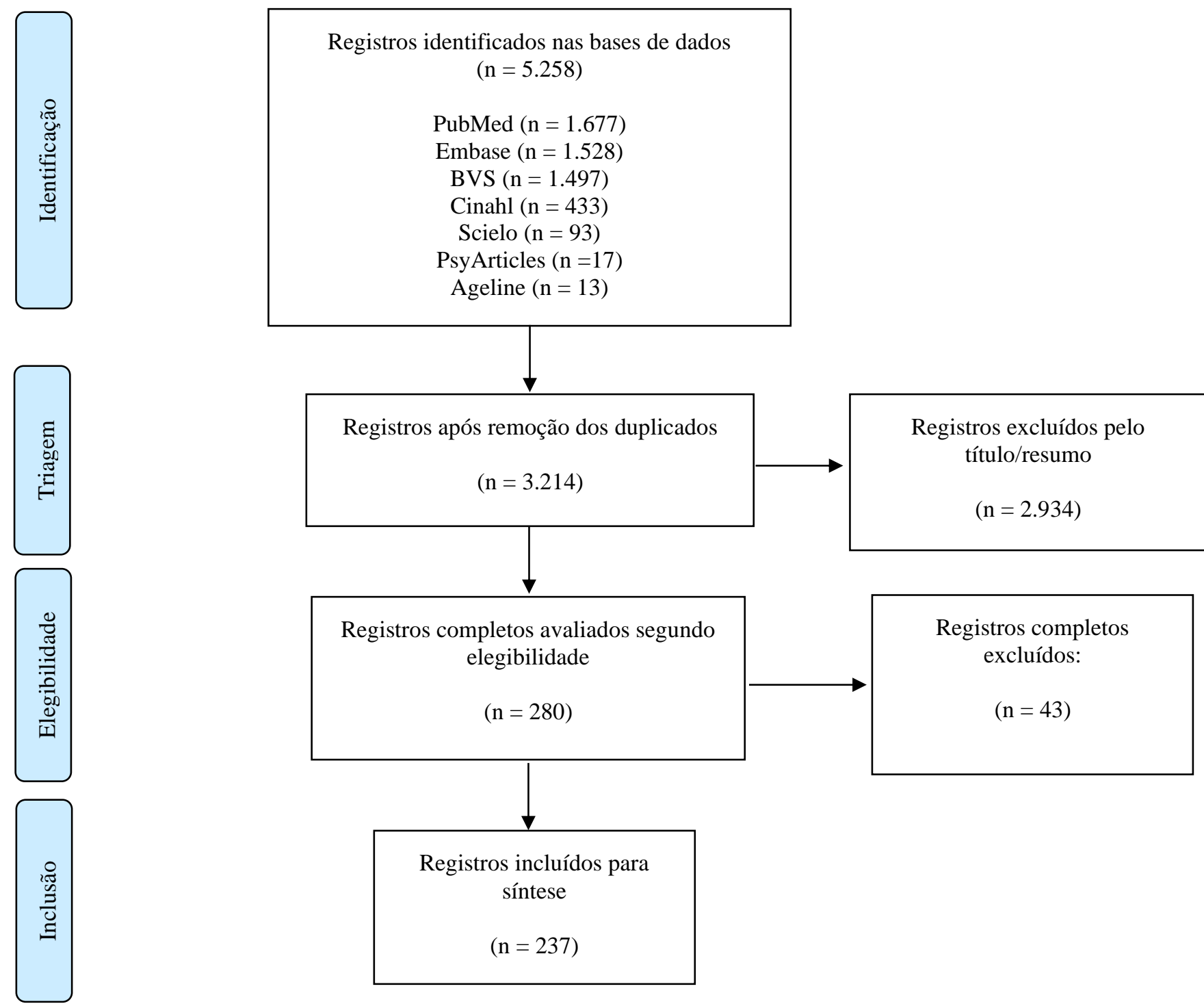

Fonte: Aline Cristina da Silva, 2020

\subsubsection{Identificadores da revisão}

Em relação aos 237 estudos analisados, 99,16\% estão disponíveis no formato artigo e apenas dois como tese de doutorado. Em sua totalidade, os artigos foram publicados em parceria de dois ou mais autores, sendo $20,68 \%$, a maioria, com quatro autores e pelo menos $5,49 \%$ com 10 ou mais autores, um deles com 16 e outro com 19 autores. A discussão sobre o crescimento das publicações científicas em diferentes áreas, bem como o número de autores, proporcionalmente, não é recente e não se esgotará mediante a esta constatação, entretanto, é 
importante ressaltar que cada área de conhecimento tem o seu modis operandi para esta atribuição, aonde sempre deve prevalecer para a definição de autoria/co-autoria a contribuição substancial para o estudo, escrita ou revisão crítica, envolvimento na análise da versão final para publicação e principalmente a responsabilidade quanto à precisão e/ou integridade dos aspectos éticos e metodológicos investigados e resolvidos.

No total, 105 periódicos (Tabela 2) estiveram envolvidos nestas publicações, sendo os mais frequentes: "Arquivos de Neuro-Psiquiatria”, "Revista de Saúde Pública”, "International Psychogeriatrics" e "Cadernos de Saúde Pública", "Dementia \& Neuropsychologia", "Brazilian Journal of Psychiatric", "PLOS ONE", "Geriatrics \& Gerontology International", "Brazilian Journal of Physical Therapy" e "International Journal of Geriatric Psychiatry", possuem entre 5 e 22 publicações em cada um deles, totalizando $41,77 \%$ das escolhas dos autores para as publicações relacionadas a esta temática.

Tabela 2. Periódicos de publicação dos estudos incluídos nesta scoping review, 2020

\begin{tabular}{|c|c|}
\hline Periódico & Quantidade de publicações \\
\hline Arquivos de Neuro-Psiquiatria & 22 \\
\hline Revista de Saúde Pública & 16 \\
\hline International Psychogeriatrics & 15 \\
\hline Cadernos de Saúde Pública & 13 \\
\hline Dementia \& Neuropsychologia & 7 \\
\hline Plos One & 6 \\
\hline Brazilian Journal of Physical Therapy & 5 \\
\hline Brazilian Journal of Psychiatry & 5 \\
\hline Geriatrics \& Gerontology International & 5 \\
\hline International Journal of Geriatric Psychiatry & 5 \\
\hline Archives of Gerontology and Geriatrics & 4 \\
\hline Einstein & 4 \\
\hline Movement Disorders & 4 \\
\hline Psicologia: Reflexão e Crítica & 4 \\
\hline Revista da Escola de Enfermagem da USP & 4 \\
\hline Trends in Psychiatry and Psychotherapy & 4 \\
\hline Clinical Gerontologist & 3 \\
\hline Journal of Voice & 3 \\
\hline Revista da Associação Médica Brasileira & 3 \\
\hline Revista Latino Americana de Enfermagem & 3 \\
\hline The Spanish Jornal of Psychology & 3 \\
\hline Acta Paulista de Enfermagem & 2 \\
\hline
\end{tabular}




\begin{tabular}{|c|c|}
\hline Aging \& Mental Health & 2 \\
\hline Aging Clinical and Experimental Research & 2 \\
\hline Alzheimer Disease and Associated Disorders & 2 \\
\hline Dementia and Geriatric Cognitive Disorders & 2 \\
\hline Health and Quality of Life Outcomes & 2 \\
\hline Journal of Afective Disorders & 2 \\
\hline Journal of the American Geriatrics Society & 2 \\
\hline Journal of the American Medical Directors Association & 2 \\
\hline Motricidade & 2 \\
\hline Paideia & 2 \\
\hline Revista Brasileira de Reumatologia & 2 \\
\hline Revista Dor & 2 \\
\hline Revista Gaúcha de Enfermagem & 2 \\
\hline São Paulo Medical Journal & 2 \\
\hline Acta Neurológica Escandinava & 1 \\
\hline Advances in Rheumatology & 1 \\
\hline Age Aging & 1 \\
\hline Aging Neuroscience & 1 \\
\hline American Academy of Neurology & 1 \\
\hline American Journal of Alzheimer's Disease \& Other Dementias & 1 \\
\hline Archives of Clinical Neuropsychology & 1 \\
\hline Archives of Clinical Psychiatry & 1 \\
\hline Arquivos Brasileiros de Cardiologia & 1 \\
\hline Biblioteca Digital USP Teses e Dissertações & 1 \\
\hline BMC Geriatrics & 1 \\
\hline Canadian Journal on Aging & 1 \\
\hline Ciência \& Saúde Coletiva & 1 \\
\hline Clinical Rehabilitation & 1 \\
\hline Clinics & 1 \\
\hline CODAS (Communication Disorders, Audiology and Swatllowing & 1 \\
\hline Comprehensive Psychiatric & 1 \\
\hline Eating and Weight Disorders & 1 \\
\hline Escola Anna Nery & 1 \\
\hline European Archives of Psychiatry and Clinical Neuroscience & 1 \\
\hline European Journal of Physical and Rehabilitation Medicine & 1 \\
\hline Experimental Aging Research & 1 \\
\hline Experimental Gerontology & 1 \\
\hline Fisioterapia Brasil & 1 \\
\hline Geriatric Nursing & 1 \\
\hline
\end{tabular}




\begin{tabular}{|c|c|}
\hline Gerontology & 1 \\
\hline International Journal of Language \& Communication Disorders & 1 \\
\hline Jornal Brasileiro de Psiquiatria & 1 \\
\hline Journal Disability and Rehabilitation & 1 \\
\hline Journal of Affective Disorders & 1 \\
\hline Journal of Aging and Health & 1 \\
\hline Journal of Aging and Physical Activity & 1 \\
\hline Journal of Alzheimer Disease & 1 \\
\hline Journal of Clinical Psycology & 1 \\
\hline Journal of Community Psychology & 1 \\
\hline Journal of Epidemiology and Community Health & 1 \\
\hline Journal of Geriatric Physical Therapy & 1 \\
\hline Journal of Pain and Symptom Management & 1 \\
\hline Journal of Psycometric Research & 1 \\
\hline Journal of Rehabilitation Medicine & 1 \\
\hline Nutrition Hospitalaria & 1 \\
\hline Parkinsonism \& Related Disorders & 1 \\
\hline Physical Therapy & 1 \\
\hline Psico-USF & 1 \\
\hline Psychology \& Neuroscience & 1 \\
\hline Psychology, Health \& Medicine & 1 \\
\hline Psycological Reports & 1 \\
\hline Repositório UFMG & 1 \\
\hline Respiratory Care & 1 \\
\hline Revista Brasileira de Cineantropometria \& Desempenho Humano & 1 \\
\hline Revista Brasileira de Enfermagem & 1 \\
\hline Revista Brasileira de Epidemiologia & 1 \\
\hline Revista Brasileira de Geriatria e Gerontologia & 1 \\
\hline Revista Brasileira de Ginecologia e Obstetricia & 1 \\
\hline Revista Brasileira de Ginecologia e Obstetrícia & 1 \\
\hline Revista Brasileira de Medicina & 1 \\
\hline Revista Brasileira de Otorrinolaringologia & 1 \\
\hline Revista Brasileire de Cineantropometria e Desempenho Humano & 1 \\
\hline Revista da Saúde Pública & 1 \\
\hline Revista de Enfermagem e Referência & 1 \\
\hline Revista Latino-Americana de Psicologia & 1 \\
\hline Scientific World Journal & 1 \\
\hline Social Psychiatry and Psychiatric Epidemiology & 1 \\
\hline Springer & 1 \\
\hline
\end{tabular}




\begin{tabular}{lc}
\hline Springer Plus & 1 \\
\hline The American Journal of Geriatric Psychiatry & 1 \\
\hline The American Journal of Occupational Therapy & 1 \\
\hline The British Journal of Psychiatry & 1 \\
\hline The Journal of Nutrition, Health and Aging & 1 \\
\hline Total & $\mathbf{2 3 7}$ \\
\hline
\end{tabular}

Fonte: Aline Cristina da Silva, 2020

Pelo menos $17,30 \%$ das publicações (41) declararam explicitamente fazer parte de outro estudo ou projeto de pesquisa, referendando o nome no estudo em questão. Além disso, foi possível identificar, que alguns autores, utilizaram a mesma amostra para análises e publicações distintas. Pelo menos 6,75\% (16) estudos, utilizaram esta estratégia, sendo que um dos autores, utilizou a mesma amostra para quatro publicações distintas.

Em relação ao ano de publicação, este estudo não contemplou corte temporal, mas de acordo com os critérios de elegibilidade, foram incluídas publicações dos últimos 32 anos, ou seja, de 1988 a 2020 (Figura 2), sendo o ano de 2018, com maior número de publicações (22) em todos os 32 anos analisados. A última década representa 64,56\% das publicações, a década dos anos 2000, representa 35,02\%, a década de 1990 não houve publicação contemplada e em relação as décadas anteriores, apenas 1 único artigo foi contemplado, no ano de 1988.

Figura 2. Linha do tempo das publicações incluídas nesta scoping review, 2020

$\begin{array}{llll}1988 & 1991 & 2001 & 2011\end{array}$

$10030153 \bigcirc 0$

Fonte: Aline Cristina da Silva, 2020

Os idiomas de publicação variaram entre inglês, português e espanhol, sendo que $86,08 \%$ foram publicados em um único idioma, 12,66\% em até dois idiomas (inglês e português ou português e espanhol) e apenas 1,27\% nos três idiomas. O idioma inglês é a preferência para publicação, representando 86,92\% e não houve publicação apenas em espanhol.

\subsection{População (População)}

Em relação à localidade, 3,80\% (9) dos estudos contemplaram outros 22 países além do Brasil, representando os continentes americano com 54,55\%, europeu com 36,36\% e asiático 
com 9,09\%. Participaram 22 dos 27 dos Estados brasileiros, incluindo o Distrito Federal. O Estado de São Paulo representou 35,44\%, seguido de Minas Gerais 16,46\%, Rio de Janeiro 9,28\% e Rio Grande do Sul 7,17\%, entretanto, 18,99\% dos estudos, não relataram explicitamente o Estado participante, sendo que 5,49\% (13) foram conduzidos em mais de um Estado. O estudo com mais diversidade regional contou com a participação de 22 Estados brasileiros. Em relação às cidades de realização dos estudos, consequentemente, a cidade de São Paulo é a mais prevalente com $24,47 \%$ de participação, seguido da omissão da informação por $21,94 \%$ dos estudos, Belo Horizonte e Rio de Janeiro com 8,02\% cada e Porto Alegre com $4,64 \%$.

A variabilidade amostral, como por exemplo, a contemplação de participantes de diferentes Estados/cidades brasileiras é bastante relevante, visto a possibilidade de considerar as diferenças culturais, entre outras características que impactam diretamente nas respostas dos participantes e consequentemente, na construção/ajustamento dos itens de um instrumento de medida. Quanto ao local de recrutamento dos participantes dos estudos, 47,26\% foram provenientes de ambulatórios, clínicas e hospitais, 20,68\% da comunidade, 16,46\% não informaram claramente o local, $10,55 \%$ de grupos, centros e associações e $8,86 \%$ da atenção primária. As instituições universitárias, principalmente as públicas e federais, tem grande relevância para a manutenção do atendimento de saúde e apoio social em âmbito nacional, bem como, é responsável pelas iniciativas de pesquisa, desenvolvimento e oferta de campo para que este tipo de estudo seja realizado.

Dos estudos analisados, compuseram as amostras, mais de 86.540 participantes, sendo 72.167 participantes brasileiros. Alguns estudos não informaram de forma objetiva o total da amostra, outros, por serem somente metodológicos, não se aplicava uma amostra. O estudo com maior quantidade amostral brasileira, contou com 5.970 participantes (DAMÁSIO et al., 2016) e o menor, com 10 participantes (BORGES et al., 2017). As discussões sobre o tamanho adequado de uma amostra são inúmeras e muito relevantes para o campo científico. O método de amostragem, por exemplo, pode variar dependendo do campo de pesquisa, entretanto, nas ciências humanas, é importante ressaltar que a amostra nunca representará perfeitamente uma população, o que significa a aceitação de uma margem de erro, conhecida estatisticamente como “erro amostral”. Torna-se então relevante, o domínio deste e outros conceitos básicos da estatística, para tomadas de decisão adequadas e uma boa condução metodológica em pesquisas, principalmente a se tratar de estudos de instrumentos de medida e evidências psicométricas. 
Dos 327 estudos analisados, pelo menos 36,29\% (86) contemplaram outras populações, que não idosos em sua amostra, sendo a menor idade relatada de 14 anos (NUNES et al., 2007) e a maior de 107 anos (ATALAIA-SILVA et al., 2008). Logo, 63,71\% (151), declaradamente, tinham sua amostra composta apenas por idosos, seja pela descrição explícita da faixa etária ou pela nomeação da amostra nos campos "Método" ou "Resultados" como "idosos". Em relação a estes estudos com a amostra exclusiva de idosos (151), o corte temporal para definição/inclusão do indivíduo considerado idoso variou, sendo $\geq 60$ anos em $62,50 \%$ dos estudos, $\geq 65$ anos em $24,34 \%$ dos estudos, $\geq 74$ anos em $1,97 \%$ e somente $\geq 80$ anos em $1,32 \%$ dos estudos. É importante ressaltar que uma pesquisa que simplesmente contempla idosos em sua amostra, não é necessariamente uma pesquisa gerontológica, basicamente, por não ter a mesma lente para compreensão do idoso, velhice e processo de envelhecimento. Entretanto, quando se trata de estudos psicométricos, o tema transcende a gerontologia e outros campos/áreas, necessitando do pesquisador/profissional uma compreensão entre as diversas ciências, que se complementam para alcançarem êxito nos desafios sociais e da saúde.

Sendo assim, um estudo psicométrico que contempla uma amplitude de idade cronológica, que não se limita a faixa etária estabelecida para o idoso, deve ser analisada atentamente para melhor compreensão do quanto este poderá atender aspectos relevantes para esta faixa etária exclusiva, considerando suas características e interesses, de modo a não generalizar algum aspecto que pode ser crucial para uma tomada de decisão por exemplo. Em relação a idade cronológica demarcada como marco da velhice no Brasil, se faz prevalente as pesquisas com o corte de 60 anos, avançando paulatinamente para 65 anos, como países desenvolvidos já tem se posicionado. Estudos exclusivos com idosos de idade mais avançada, ainda são precários e essenciais para maior conhecimento e domínio das diferenças dessa fase da vida.

Os estudos contemplaram amostras em fases distintas do envelhecimento, como: adolescentes, adultos e idosos, e com diferentes características: indivíduos saudáveis, com dor, institucionalizados, estudantes, aposentados, com depressão, queixa vocal, hospitalizados, hipertensos, climatéricas, habilitados para direção de veículo automotivo, frágil, sarcopênico, com baixo nível de escolaridade, entre outros. Vale ressaltar, o grande interesse em grupos com Demência, Doença de Alzheimer, Doença de Parkinson, Comprometimento Cognitivo Leve, queixas e/ou déficits cognitivos, pelo menos $27,85 \%$ dos estudos, tiveram como alvo, amostras com essas características. Mais de $50 \%$ do total das amostras são mulheres, mais de $29 \%$ são homens e pelo menos $20 \%$ não declarou a variável gênero/sexo explicitamente, ou seja, não foi 
uma variável de interesse para os estudos em questão. Pelo menos nove estudos foram dedicados a amostras totalmente femininas, aonde as variáveis latentes de interesse foram, atividade física e prontidão para atividade física, qualidade de vida e qualidade de vida na peri/menopausa/climatério e disfunção sexual. Apenas dois estudos foram realizados com amostras totalmente masculinas, sendo as variáveis latente de interesse, o uso de álcool e atividade física.

\subsubsection{Instrumentos Psicossociais}

Dentre os 237 estudos analisados, foram identificados 258 instrumentos alvo com algum tipo de evidência psicométrica publicado. Desconsiderando os instrumentos repetidos e variações do mesmo instrumento (devido a quantidade de itens por exemplo), foram identificados 192 instrumentos considerados validados para a população idosa brasileira. Foram identificados, por descrição explícita nos estudos, seis protocolos/baterias sendo, dois com foco em avaliação neuropsicológica (DE PAULA et al., 2010; DE PAULA et al., 2013), dois com foco em aptidão/independência funcional (VIRTUOSO JUNIOR et al., 2011), um com foco em transtornos de comunicação (FONSECA et al., 2008) e um com foco em atividades de vida diária (PAES et al., 2017).

Muitos instrumentos apresentam variação de versões mais curtas ou adaptados para populações mais específicas, por exemplo o Geriatric Depression Scale, que tem versões com quantidades de itens diferentes, ou o Quality of Life Scale que tem uma versão somente para indivíduos com Alzheimer. É importante se atentar a estes detalhes para compreender a abrangência de utilização do instrumento, visto a população ao qual foi validado e à população ao qual será destinado. Foram criadas subcategorias para agrupamento dos instrumentos identificados, a fim de facilitar a busca e escolha do instrumento de interesse para cada necessidade específica. Para tanto, foram consideradas algumas premissas, descritas abaixo.

Um mesmo instrumento pode ser relatado na literatura por diferentes nomes, acrônimos ou abreviações, por este motivo, foi necessária uma padronização da identificação. O idioma inglês foi escolhido como primeira opção para a descrição do nome, exceto quando o instrumento foi desenvolvido em português e não apresentou tradução quando publicado em inglês. Baseou-se também nas versões encontradas na literatura, o que não significa que foi escolhida a versão original ou mais utilizada, e sempre que informado no estudo de origem ou encontrado na literatura, o acrônimo foi disponibilizado para facilitar a comunicação. 
Mais de 250 variáveis latentes foram mencionadas em todos os estudos como alvo destes instrumentos, sendo as mais prevalentes, desordens cognitivas, qualidade de vida e depressão. Assim como a identificação, a variável latente é descrita diferentemente em cada estudo, o que dificulta a compreensão do que está se propondo medir, além de emparelhar conceitos terminológicos entre os profissionais, acadêmicos e outros que tenham interesse nesta temática. A variável latente exposta neste estudo, foi identificada e reproduzida estritamente como no estudo de origem, incluído para a síntese analítica, e quando necessário, devido à falta de clareza, foi adaptada/definida pela autora, que se baseou em buscas aleatórias na literatura acadêmica. A tabela 3 refere-se à subcategoria: Ansiedade, Depressão e Estresse, que destaca os instrumentos identificados para estes transtornos especificamente. Foram contemplados 11 instrumentos nesta subcategoria, sendo seis destinados à mensuração da depressão.

Tabela 3. Instrumentos para idosos brasileiros - Subcategoria: Ansiedade, Depressão e Estresse, 2020

\begin{tabular}{|c|c|c|c|}
\hline Instrumento & Acrônimo & Variável latente & Autor, (Ano) \\
\hline $\begin{array}{l}\text { Center for Epidemiologic } \\
\text { Studies Depression Scale }\end{array}$ & CES-D & Depressão & Batistoni et al., (2007) \\
\hline Depression Anxiety Stress Scale & $\begin{array}{l}\text { DASS; } \\
\text { DASS- } \\
\text { Depressão }\end{array}$ & $\begin{array}{l}\text { Ansiedade, Estresse e } \\
\text { Depressão }\end{array}$ & $\begin{array}{l}\text { Sardá Jr et al., (2008); } \\
\text { Vignola et al., (2014) }\end{array}$ \\
\hline Depression Scale for the Elderly & DSE & Depressão & Giavoni et al., (2008) \\
\hline Geriatric Anxiety Inventory & GAI-BR & Ansiedade & Massena et al., (2015) \\
\hline Geriatric Depression Scale & $\begin{array}{l}\text { GDS-1; } \\
\text { GDS-4; } \\
\text { GDS-10; } \\
\text { GDS-15; } \\
\text { GDS-30; }\end{array}$ & Depressão & $\begin{array}{l}\text { Alvarenga et al., (2012); } \\
\text { Castelo et al., (2010); } \\
\text { Chachamovich et al., (2010); } \\
\text { Costa et al., (2006); } \\
\text { Paradela et al., (2005); } \\
\text { Pinho et al., (2010); } \\
\text { Vargas et al., (2007) }\end{array}$ \\
\hline $\begin{array}{l}\text { Geriatric Emotional Assessment } \\
\text { of Pain }\end{array}$ & GEAP-b & $\begin{array}{l}\text { Depressão Induzida } \\
\text { pela Dor }\end{array}$ & Almeida et al., (2017) \\
\hline Hamilton Anxiety Rating Scale & HAM-A & Ansiedade & Kummer et al., (2010) \\
\hline Liebowitz Social Anxiety Scale & LSAS & Ansiedade Social & Kummer et al., (2008) \\
\hline $\begin{array}{l}\text { Montgomery-Åsberg } \\
\text { Depression Rating Scale }\end{array}$ & MADRS & Depressão & $\begin{array}{l}\text { Portugal et al., (2012); } \\
\text { Silberman et al., (2006) }\end{array}$ \\
\hline Patient Health Questionnaire & $\begin{array}{l}\text { PHQ-2 } \\
\text { PHQ-9 }\end{array}$ & Depressão & $\begin{array}{l}\text { Chagas et al., (2011); } \\
\text { Chagas et al., (2013) }\end{array}$ \\
\hline Perceived Stress Scale & PSS & Estresse & Luft et al., (2007) \\
\hline
\end{tabular}

Fonte: Aline Cristina da Silva, 2020 
Referente aos instrumentos para depressão, destaca-se o GDS, como alvo em pelo menos oito estudos, todos eles com população exclusivamente de idosos. O estudo de Castelo et al., (2010) objetivou a validação das versões GDS-1, GDS-4, GDS-10, GDS-15 e GDS-30, além da definição do ponto de corte para a identificação do transtorno em cada uma delas. $\mathrm{O}$ estudo de Costa et al., (2006) trabalhou com o critério de inclusão $\geq 75$ anos em uma quantidade amostral considerável, $\mathrm{N}=392$, porém, é o estudo menos atual contemplado nesta análise, para esta variável. A maior amostra foi de Costa et al., (2008), com um N=1.172. Já o estudo de Pinho et al., (2010), considerou uma amostra de idosos com doença arterial coronariana. Ainda sobre os instrumentos de depressão, importante destacar GEAP-b. Este instrumento se destina especificamente a depressão induzida pela dor, o estudo de Almeida et al., (2017) teve como amostra, exclusivamente idosos com dor crônica, além de ser o estudo mais recente entre os instrumentos para depressão.

Em relação aos instrumentos de ansiedade, foram identificados quatro, sendo três exclusivos para mensuração da variável e o quarto, um instrumento multidimensional. Destaque para o estudo de Massena et al., (2015), que além de mais recente, foi o único que trabalhou com uma amostra exclusiva de idosos, com o instrumento GAI-BR. Os estudos de Kummer et al., (2008; 2010), com os instrumentos LSAS e HAM-A contemplou amostras de indivíduos com Doença de Parkinson. Por fim, o instrumento DASS, alvo dos estudos de Sardá Jr et al., (2008) e Vignola et al., (2014), apresenta base conceitual diferente dos demais, referenciando o modelo tripartido de ansiedade e depressão, aonde a desordem do afeto é um continuum entre ansiedade, depressão e estresse, este modelo foi proposto por Clark e Watson (1991) se propõem a avaliar estresse nesta população, além de, simultaneamente, ansiedade e depressão. E o instrumento PSS de Luft et al., (2007) foi traduzido, adaptado e validado com uma amostra de 76 idosos. A tabela 4 refere-se à subcategoria: Atividade Física, Capacidade Funcional e Mobilidade. Foram categorizados 48 instrumentos, que se propõem avaliar uma ampla classe de ações voluntárias, que envolvem não somente o grau de preservação para realizar uma atividade cotidiana, mas também, a capacidade de resposta da musculatura esquelética. 
Tabela 4. Instrumentos para idosos brasileiros - Subcategoria: Atividade Física, Capacidade Funcional e Mobilidade, 2020

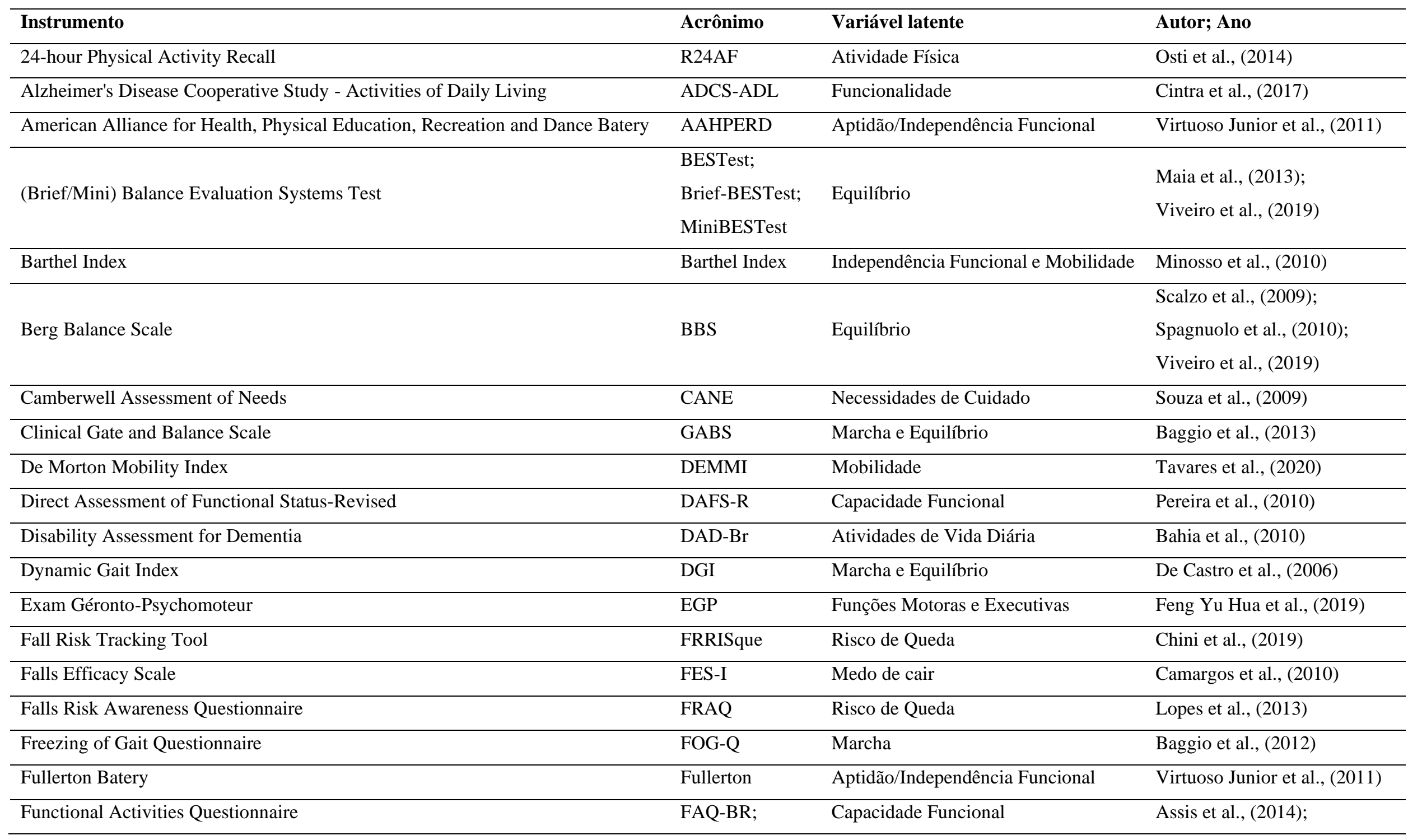




\begin{tabular}{|c|c|c|c|}
\hline & P-FAQ & & Jomar et al., (2018); \\
\hline General Activities of Daily Living Scale & GADL & Atividades de Vida Diária & Paula et al., (2014) \\
\hline Godin-Shephard Leisure-Time Physical Activity Questionnaire & GSLTPAQ & Tempo de Lazer e Atividade Física & João et al., (2015) \\
\hline Habitual Physical Activity & HPA & Atividade Física & Florindo et al., (2004); \\
\hline Human Activity Profile & HAP & Atividade Física & $\begin{array}{l}\text { Bastone et al., (2014); } \\
\text { Souza et al., (2006) }\end{array}$ \\
\hline Incremental Shuttle Walk test & ISWT & Equilíbrio & Spagnuolo et al., (2010) \\
\hline Instrument for classifying elderly people regarding their capacity for self-care & ESCC & Funcionalidade e Autonomia & Almeida et al., (2008) \\
\hline Johns Hopkins Fall Risk Assessment Tool & JH-FRAT & Risco de Queda & $\begin{array}{l}\text { Martinez et al., (2016); } \\
\text { Martinez et al., (2019) }\end{array}$ \\
\hline Late-Life Function and Disability Instrument & LLFDI-BR & Funcionalidade e Incapacidade & De Almeida et al., (2016) \\
\hline Life-Space Assessment & LSA & Mobilidade & $\begin{array}{l}\text { Curcio et al., (2013); } \\
\text { Garcia et al., (2018); } \\
\text { Simoes et al., (2018) }\end{array}$ \\
\hline Lindop Parkinson's Disease Mobility Assessment & LPA & Mobilidade & Santos et al., (2017) \\
\hline List Advanced Activities of the Daily Living & AADLs & Atividade de Vida Diária & Dias et al., (2018) \\
\hline Londrina Activities of Daily Living Protocol & Londrina ADL & Atividades de Vida Diária & Paes et al., (2017) \\
\hline Modified Parkinson Activity Scale & PASm & Mobilidade & Santos et al., (2017) \\
\hline Neighborhood Environment Walkability Scale & NEWS & Ambiente para Atividade Física & Florindo et al., (2012) \\
\hline Non-Motor Symptoms Scale & NMSS & Sintomas Não Motores & Martinez-Martin et al., (2009) \\
\hline Online Questionnaire for Fall Risk Assessment & NI & Risco de Queda & Silveira et al., (2018) \\
\hline Parkinson Activity Scale & PAS & Nível de Atividade & Santos et al., (2015) \\
\hline Parkinson's Fatigue Scale & PFS & Fadiga & Kummer et al., (2011) \\
\hline Physical Activity Readiness Questionnaire & PAR-Q & Prontidão para Atividade Física & Maranhao Neto et al., (2013) \\
\hline
\end{tabular}




\begin{tabular}{|c|c|c|c|}
\hline Profile of Function and Impairment Level Experience with Parkinson Disease & PROFILE PD & $\begin{array}{l}\text { Alterações Corporais em Indivíduos } \\
\text { com Parkinson }\end{array}$ & Swarowsky et al., (2017) \\
\hline Program on Research for Integrating Services for the Maintenance of Autonomy & PRISMA-7 & Independência Funcional & Saenger et al., (2018) \\
\hline Rating of Perceived Exertion & RPE & Dependência para o Autocuidado & De Souza et al., (2018) \\
\hline Scale of Independence in Activities of Daily Living & KATZ Index & Atividades de Vida Diária & $\begin{array}{l}\text { Lino et al., (2008); } \\
\text { Ferretti-Rebustini et al., (2015) }\end{array}$ \\
\hline Scales for Outcomes in Parkinson's Disease-Autonomic Questionnaire & SCOPA-AUT & Disfunção autonômica & Carod-Artal et al., (2009) \\
\hline Short Physical Performance Battery & SPPB & Desempenho Físico & $\begin{array}{l}\text { Freire et al., (2012); } \\
\text { Perracini et al., (2020) }\end{array}$ \\
\hline Southampton Assessment of Mobility & SAM & Mobilidade em Idosos com Demência & Pereira et al., (2006) \\
\hline Timed Up and Go & $\begin{array}{l}\text { TUG; } \\
\text { TUG-ABS }\end{array}$ & Funcionalidade Marcha & $\begin{array}{l}\text { Da Silva et al., (2017); } \\
\text { Spagnuolo et al., (2010) }\end{array}$ \\
\hline Trunk Mobility Scale & TMS & Rigidez Axial & Franco et al., (2011) \\
\hline Vestibular Disorders Activities of Daily Living Scale & VADL & Tontura e Equilíbrio & Ricci et al., (2014) \\
\hline
\end{tabular}

NI - Não Informado

Fonte: Aline Cristina da Silva, 2020 
Nesta subcategoria, 57,14\%, ou seja, 36 dos 63 estudos, utilizaram uma amostra exclusivamente de idosos. Vale ressaltar que 12 das 36, com características específicas, como o estudo de Bahia et al., (2010), com o instrumento DAD-Br, que se dedicou a uma amostra de idosos com Doença de Alzheimer. O estudo de Pereira et al., (2006), com o instrumento SAM, utilizou uma amostra apenas de idosos com Demência. Os estudos de Cintra et al., (2017) e Pereira et al., (2010), com os respectivos instrumentos ADCS-ADL e DAFS-R, utilizaram uma amostra de idosos com Doença de Alzheimer e com Comprometimento Cognitivo Leve.

Garcia et al., (2018), utilizou uma amostra de idosos com obstrução pulmonar crônica, para o instrumento LSA. Os estudos de Bastone et al., (2014), Maranhão Neto et al., (2013), De Souza et al., (2018) e Virtuoso Júnior et al., (2011), utilizaram uma amostra exclusivamente feminina para os respectivos instrumentos HAP, PAR-Q, RPE e AAHPERD e Fullerton. O estudo de Viveiro et al., (2019), com os instrumentos BBS; BESTest, Mini-BESTest; BriefBESTest, utilizou uma amostra de idosos institucionalizados. O estudo de Perracini et al., (2020), com o instrumento SPPB, utilizou uma amostra de idosos frágeis. E por fim, o estudo de Ricci et al., (2014), com o instrumento VADL, utilizou uma amostra de idosos com desordem vestibular. É importante se atentar as particularidades das amostras ao que os estudos de evidência psicométrica se dedicaram e assim escolher o instrumento de forma mais adequada de acordo com a população que será avaliada.

Em relação aos outros 27 estudos que contemplaram também a população adulta, pelo menos 12 estudos, se dedicaram à indivíduos com Doença de Parkinson, foram estes: Baggio et al., (2013); Baggio et al., (2012); Carod-Artal et al., (2009); Da Silva et al., (2017); Franco et al., (2011); Kummer et al., (2011); Maia et al., (2013); Martinez-Martin et al., (2009) Swarowsky et al., (2017); Santos et al., (2017); Santos et al., (2015); Scalzo et al., (2009) e respectivamente os instrumentos: GABS; FOG-Q; SCOPA-AUT; TUG-ABS; TMS; PFS; BESTest e MiniBESTest; NMSS; PROFILE PD; PASm e LPA; PAS; BBS. O estudo mais antigo desta subcategoria é de Florindo et al., (2004), com o instrumento HAP e os mais atuais são de Tavares et al., (2020) e Perracini et al., (2020) com os instrumentos DEMMI e SPPB. Há quase duas décadas, com exceção dos anos de 2005 e 2007, todos os anos houve uma ou mais publicação sobre esta temática, o que demonstra a relevância e necessidade de aprofundamento e apropriação para os estudiosos e profissionais da gerontologia e geriatria.

A tabela 5, refere-se à subcategoria Inteligência e Cognição, estão reunidos 47 instrumentos que buscam, em sua maioria, identificar declínio em algum aspecto da 
inteligência/cognição. Este construto é complexo e muito discutido, por se tratar de uma das variáveis mais importantes para a humanidade. Seu desempenho está ligado a funções cerebrais e a experiências ambientais ao longo da vida. Sendo que algumas das capacidades desenvolvidas, podem sofrer declínio até a velhice, por isso, a medição e compreensão deste construto se torna tão relevante ao logo da vida. 
Tabela 5. Instrumentos para idosos brasileiros - Subcategoria: Inteligência e Cognição, 2020

\begin{tabular}{|c|c|c|c|}
\hline Instrumento & Acrônimo & Variável latente & Autor; Ano \\
\hline (Mini) Addenbrooke's Cognitive Examination-Revised & $\begin{array}{l}\text { ACE-R; } \\
\text { M-ACE }\end{array}$ & Desordens Cognitivas & $\begin{array}{l}\text { Cesar et al., (2017); } \\
\text { Miranda et al., (2018); } \\
\text { Sobreira et al., (2015) }\end{array}$ \\
\hline Ascertaining Dementia Interview & AD8-Brazil & Desordens Cognitivas & Correia et al., (2011) \\
\hline Australian National University-Alzheimer's Disease Risk Index & ANU-ADRI & Fator de Risco para Alzheimer & Borges et al., (2017) \\
\hline Boston Naming Test & BNT & Distúrbios de Fala & Leite et al., (2017) \\
\hline Brief Neuropsychological Protocol & Protocol & Desordens Cognitivas & De Paula et al., (2010) \\
\hline Cambridge Cognitive Examination Revised-Brazilian & BR-CAMCOG-R & Desordens Cognitivas & Paradela et al., (2009) \\
\hline Categorization Working Memory Span Task & CWMS Task & Memória de Trabalho & Brum et al., (2017) \\
\hline Clinical Dementia Rating & CDR & Desordens Cognitivas & $\begin{array}{l}\text { Macedo Montano et al., } \\
(2005) ; \\
\text { Maia et al., (2006) }\end{array}$ \\
\hline Clock Drawing Test & CDT & Desordens Cognitivas & $\begin{array}{l}\text { Atalaia-Silva et al., (2008); } \\
\text { Cecato et al., (2012); } \\
\text { Lourenço et al., (2008) }\end{array}$ \\
\hline Cognitive Abilities Screening Instrument & CASI-S & Desordens Cognitivas & $\begin{array}{l}\text { Damasceno et al., (2005); } \\
\text { De Oliveira et al., (2016) }\end{array}$ \\
\hline Confusion Assessment Method & CAM & Delírio & Fabbri et al., (2001) \\
\hline Consortium to Establish a Registry for Alzhmeir's Disease & CERAD & Desordens Cognitivas & Bertolucci et al., (2001) \\
\hline Delirium Rating Scale-Revised & DRS-R-98 & Delírio & De Negreiros et al., (2008) \\
\hline Dementia Rating Scale & DRS & Desordens Cognitivas & Jacinto et al., (2012) \\
\hline Episodic Autobiographic Memory Interview & EAMI & Memória Episódica Autobiográfica & Rodrigues et al., (2015) \\
\hline Executive Function Performance Test & EFPT & Função Executiva & Neubern, (2018) \\
\hline
\end{tabular}




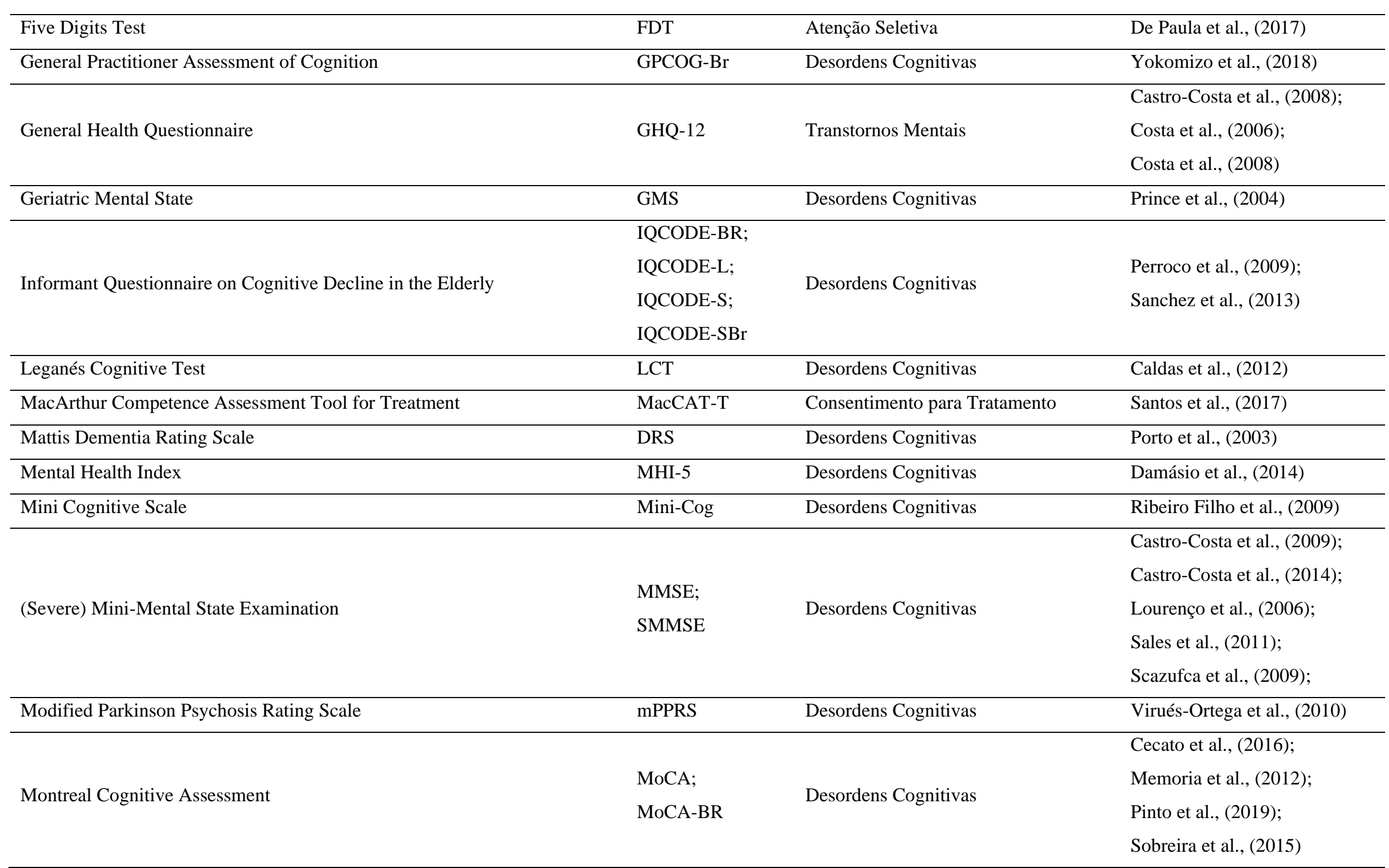




\begin{tabular}{|c|c|c|c|}
\hline Multifactorial Memory Questionnaire & MMQ & Metamemória & Simon et al., (2016) \\
\hline Neuropsychiatric Inventory (Clinician Rating Scale) & $\begin{array}{l}\text { NPI; } \\
\text { NPI-C }\end{array}$ & Distúrbios Neuropsiquiátricos & $\begin{array}{l}\text { Camozzato et al., (2008); } \\
\text { Stella et al., (2013) }\end{array}$ \\
\hline $\begin{array}{l}\text { Parkinson's Disease Impulsive-Compulsive Disorders Questionnaire - } \\
\text { Current Short }\end{array}$ & QUIP-CS & Desordens Cognitivas & Krieger et al., (2017) \\
\hline Philadelphia Brief Assessment of Cognition & PBAC & Desordens Cognitivas & Pereira et al., (2012) \\
\hline Premorbid Cognitive Abilities Scale & PCAS & Habilidades Cognitivas Pré-Mórbidas & Apolinario et al., (2013) \\
\hline $\begin{array}{l}\text { Neuropsychological } \\
\text { Assessment Protocol }\end{array}$ & Protocol & Desordens Cognitivas & De Paula et al., (2013) \\
\hline Rivermead Behavioural Memory Test & RBMT & Memória & Yassuda et al., (2010) \\
\hline Rowland Universal Dementia Assessment Scale & RUDAS-BR & Desordens Cognitivas & De Araujo et al., (2018) \\
\hline Screening test for Alzheimer's Disease with Proverbs & STADP & Funções Executivas & Santos et al., (2009) \\
\hline Self-Reporting Questionnaire & SRQ-20 & Desordens Cognitivas & Scazufca et al., (2009) \\
\hline Short Cognitive Performance Test & SKT & Desordens Cognitivas & Flaks et al., (2006) \\
\hline Short Form of the Wechsler-III Scale & SF8 & Inteligência & Banhato et al., (2010) \\
\hline Short Psychiatric Evaluation Schedule & SPES-15 & Desordens Psiquiátricas & Blay et al., (1988) \\
\hline Stick Design Test & NI & Habilidade Visuoespacial & De Paula et al., (2013) \\
\hline Taylor Complex Figure Task & TCFT & Habilidade Visuoespacial & De Paula et al., (2016) \\
\hline The Picture Free and Cued Selective Reminding with Immediate Recall & pFCSRT-IR & Memória episódica & Zibetti et al., (2019) \\
\hline Token Test & TT & Compreensão Verbal & Paula et al., (2012) \\
\hline Tower of London Test & TOL & Função Executiva & De Paula et al., (2012) \\
\hline
\end{tabular}

NI - Não Informado

Fonte: Aline Cristina da Silva, 2020 
Dos 47 instrumentos deste subgrupo, pelo menos 28 são destinados a rastreio, triagem, detecção precoce de distúrbios cognitivos, processos demenciais e buscam de uma forma rápida e com baixo custo identificar transtornos mentais e cognitivos como Demência, Comprometimento Cognitivo Leve, Doença de Alzheimer, Doença de Parkinson, entre outros. Estes instrumentos se diferenciam em vários aspectos, como tipo de pergunta, tipo de resposta, quantidade de itens, tempo de aplicação, tipo de correção, pontuação, sensibilidade e especificidade para a população designada. Serão comentados abaixo, os instrumentos com pelo menos duas ou mais fontes de evidências psicométricas capturadas para esta análise, não necessariamente os mais relevantes e adequados para avaliação da variável latente em questão. Sendo assim, antes da escolha para utilização, quando necessário avaliar algum aspecto de inteligência e/ou cognição, sugere-se uma leitura de todas as referências disponíveis nesta tabela, para análise e definição adequada do aplicador.

O MMSE é sem dúvida o instrumento mais utilizado para fins de rastreio cognitivo, mencionado em literatura nacional e internacional. Nesta análise, foi alvo de cinco estudos, entretanto, está presente em pelo menos outros 86 dos 237 estudos, relatado no método e resultados dos estudos, seja como parte dos critérios de elegibilidade ou para composição das variáveis analisadas. Os estudos de Castro-Costa et al., (2009); Castro-Costa et al., (2014); Lourenço et al., (2006); Sales et al., (2011); Scazufca et al., (2009); se dedicaram a gerar a evidências de validade do MEEM e estabelecer pontos de corte adequados para a população idosa. Dos cinco estudos, três consideraram idosos, com idade a partir de 60 e outros dois, com idade a partir de 65 anos. Foi relatada uma amplitude de idade de até 102 anos, bastante considerável para estimativas entre idosos mais velhos. $\mathrm{O}$ estudo com maior amostra contou com 1.933 idosos e tece comentários importantes sobre a influência das características sociodemográficas em relação ao instrumento, principalmente no que tange educação formal e idosos mais velhos, ressaltando o risco de uma superestimação da classificação de demência (SCAZUFCA et al., 2009).

Outro instrumento para rastreio cognitivo, alvo de quatro estudos, Cecato et al., (2016); Memoria et al., (2012); Pinto et al., (2019); Sobreira et al., (2015) aqui considerados, foi o MoCA. A grande diferença relatada entre este e o MMSE, é que o MoCA parece ter mais precisão para identificar Comprometimento Cognitivo Leve. Todos os quatro estudos, se dedicaram a alguma evidência de validade, sendo que o estudo com maior amostra contou com 229 idosos, a partir de 65 anos (PINTO et al., 2019), já o estudo com menor amostra, contou com 79 indivíduos, adultos e idosos, de 28 a 81 anos, sendo o único que contemplou outros 
indivíduos além de idosos (SOBREIRA et al., 2015). O teste GHQ-12, foi capturado por meio de três estudos (CASTRO-COSTA et al., 2008; COSTA et al., 2006; COSTA et al., 2008) do mesmo principal autor, todos fazem parte do projeto Bambuí Health Aging Study (BHAS), um estudo longitudinal de base populacional de idosos, realizado na cidade de Bambuí-MG, com linha de base de coorte em 1997. Este instrumento é utilizado para triagem de transtornos mentais, sendo que os estudos aqui contemplados, se dedicaram a analisar evidências de validade e confiabilidade do instrumento em questão. O CDT ganha destaque por ser alvo de três estudos desta análise (ATALAIA-SILVA et al., 2008; CECATO et al., 2012; LOURENÇO et al., 2008). As evidências de validade reunidas nestes três estudos tratam das evidências de validade de conteúdo (tradução e adaptação) e estrutura interna. Também utilizado para rastreio cognitivo, estes estudos foram realizados exclusivamente com idosos, sendo o estudo de Cecato et al., (2012) o mais recente e com maior quantidade amostral (426). Também presente em três estudos contemplados nesta análise (CESAR et al., 2017; MIRANDA et al., 2018; SOBREIRA et al., 2015), o ACE, seja a versão ACE-R ou M-ACE, geralmente tem sido utilizado para rastreio cognitivo de Demência e Comprometimento Cognitivo Leve em indivíduos/idosos com outros distúrbios, como Doença de Parkinson ou Doença de Alzheimer. Os estudos aqui capturados, contaram com amostras de adultos e idosos ou exclusivamente idosos, reunindo evidências de validade e confiabilidade.

O IQCODE e suas versões, foram analisados em dois estudos aqui contemplados (PERROCO et al., 2009; SANCHEZ et al., 2013) e seu diferencial está ligado à sua aplicação, este instrumento é utilizado para triagem/rastreio cognitivo por meio de um informante/cuidador. Ambos os estudos foram realizados exclusivamente com idosos, reunindo evidências de validade. O CDR tem o objetivo de classificar as funções cognitivas dos idosos, assim como o IQCODE, também é um instrumento onde as repostas são coletadas de familiares, cuidadores ou outros que convivem diariamente com o idoso. Os estudos de Macedo Montano et al., (2005) e Maia et al., (2006), reuniram evidências de validade e confiabilidade, com amostras compostas de adultos e idosos.

Por fim, o CASI-S foi analisado nos estudos de Damasceno et al., (2005) e De Oliveira et al., (2016), reunindo evidências de validade e confiabilidade. Este instrumento tem por objetivo o rastreio e a classificação de demência, sendo estudado em amostras de adultos e idosos, com diagnóstico de Doença de Alzheimer e Demência, a amplitude de idade relatada variou entre 40 e 88 anos. A tabela 6, trata da subcategoria: Saúde, Bem-estar e Qualidade de Vida e buscou integrar instrumentos que se preocupam com qualquer aspecto, positivo ou 
negativo, que possa impactar a vida do idoso. São construtos, por vezes, multidimensionais, que por referir-se as experiências pessoais, podem ser avaliados em aspectos globais ou domínios específicos, como trabalho, família, interação social, saúde física ou emocional, sexualidade, espiritualidade, entre outros, incluindo então medidas cognitivas e emocionais. É uma das subcategorias mais complexas e amplas deste estudo. Foram categorizados 74 instrumentos, sendo o de maior destaque o World Health Organization Quality of Life, mais conhecido como WHOQOL, que conta com diferentes versões e foi alvo de nove estudos diferentes. 
Tabela 6. Instrumentos para idosos brasileiros - Subcategoria: Saúde, Bem-estar e Qualidade de Vida, 2020

\begin{tabular}{|c|c|c|c|}
\hline Instrumento & Acrônimo & Variável latente & Autor; (Ano) \\
\hline Adaptation Partnership Growth Affection Resolve & APGAR & Funcionalidade familiar & Da Silva et al., (2014) \\
\hline Alcohol Use Disorders Identification Test & AUDIT & Uso de álcool & De Oliveira et al., (2011) \\
\hline $\begin{array}{l}\text { American Speech-Language-Hearing Association Functional Assessment of } \\
\text { Communication Skills }\end{array}$ & ASHA FACS & Comunicação & De Carvalho et al., (2008) \\
\hline Apathy Inventory & AI & Apatia & Stella et al., (2013) \\
\hline Attitudes to Aging Questionnaire & AAQ & $\begin{array}{l}\text { Atitudes em relação ao } \\
\text { envelhecimento }\end{array}$ & Chachamovich et al., (2008) \\
\hline Back Beliefs Questionnaire & BBQ & Dor Lombar Aguda & Teixeira et al., (2020) \\
\hline Beliefs about Emotions Scale & BES & Crenças sobre Emoções & Mograbi et al., (2018) \\
\hline Bem Sex Role Inventory & BSRI & $\begin{array}{l}\text { Masculinidade e } \\
\text { Feminilidade }\end{array}$ & Ahmed et al., (2016) \\
\hline Brazilian Version of the California Older Person's Pleasant Events Schedule & OPPES-BR & Atividades Prazerosas & Ferreira et al., (2015) \\
\hline Cervantes Scale & CS & $\begin{array}{l}\text { Qualidade de Vida na } \\
\text { Peri/Menopausa }\end{array}$ & Lima et al., (2012) \\
\hline Control, Autonomy, Self-Realization and Pleassure & CASP-19 & Qualidade de Vida & $\begin{array}{l}\text { Lima et al., (2014); } \\
\text { Neri et al., (2018) }\end{array}$ \\
\hline Difficulties in Emotion Regulation Scale & DERS & Regulação Emocional & Miguel et al., (2017) \\
\hline Driving Cognitions Questionnaire & DCQ & Motivação para Dirigir & OliSan et al., (2015) \\
\hline Early Trauma Inventory Self Report-Short Form & ETISR-SF & Experiências Traumáticas & Osório et al., (2013) \\
\hline Edmonton Symptom Assessment System & ESAS-r & $\begin{array}{l}\text { Sintomas em Pacientes } \\
\text { Paliativos }\end{array}$ & Monteiro et al., (2013) \\
\hline Elderly Quality of Life Index & EQoLI & Qualidade de Vida & Paschoal et al., (2008) \\
\hline Escala Fatorial de Socialização & EFS & Socialização & Nunes et al., (2007) \\
\hline
\end{tabular}




\begin{tabular}{|c|c|c|c|}
\hline Exercise Benefits/Barriers Scale & EBBS & $\begin{array}{l}\text { Percepção sobre Prática } \\
\text { de Atividade Física }\end{array}$ & Victor et al., (2001) \\
\hline Fatigue Severity Scale & FSS & Fadiga & Valderramas et al., (2012) \\
\hline Fear Avoidance Beliefs Questionnaire & FABQ-Brazil & Dor lombar & De Abreu et al., (2008) \\
\hline Food Frequency Questionnaire & QFA & Dieta & Molina et al., (2013) \\
\hline General Self-Efficacy Scale & GSES & Auto-Eficácia & Damasio et al., (2016) \\
\hline Geriatric Pain Measure & GPM & Dor & $\begin{array}{l}\text { Gambaro et al., (2009); } \\
\text { Motta et al., (2015) }\end{array}$ \\
\hline Herth Hope Scale & HHS & Esperança de Vida & Orlandi et al., (2012) \\
\hline Instrumento de Avaliação da Saúde do Idoso & IASI & Saúde Geral & Pedreira et al., (2016) \\
\hline Manchester Foot-Pain Disability Index & MFPDI & Dor no Pé & Ferrari et al., (2008) \\
\hline Meaning in Life Questionnaire & MLQ & Propósito de Vida & Damásio et al., (2015) \\
\hline Medical Outcomes Study Social Support Survey & MOS-SSS & Apoio Social & Zucoloco et al., (2019) \\
\hline Michigan Alcoholism Screening Test - Geritatric Version & MAST-G & $\begin{array}{l}\text { Abuso/Dependência de } \\
\text { Álcool }\end{array}$ & Kano et al., (2014) \\
\hline Minnesota Living with Heart Failure Questionnaire & LHFQ & $\begin{array}{l}\text { Qualidade de Vida em } \\
\text { idosos com Insuficiência } \\
\text { Cardíaca }\end{array}$ & Saccomann et al., (2007) \\
\hline Multidimensional Assessment of Older People in Primary Care & AMPI-AB & $\begin{array}{l}\text { Avaliação Geriátrica } \\
\text { Ampla }\end{array}$ & Saraiva et al., (2020) \\
\hline Multidimensional Body-Self Relations Questionnaire-Appearance Scales & MBSRQ-AS & Imagem Corporal & Laus et al., (2020) \\
\hline Nottingham Health Profile & NHP & $\begin{array}{l}\text { Qualidade de Vida em } \\
\text { idosos com Doença de } \\
\text { Parkinson e Hemiplegia }\end{array}$ & Teixeira-Salmela et al., (2004) \\
\hline Oral Health Risk Assessment & OHRA & Problemas de Saúde Bucal & Machado et al., (2012) \\
\hline
\end{tabular}




\begin{tabular}{|c|c|c|c|}
\hline Pain Assessment Checklist for Seniors With Limited Ability to Communicate & PACSLAC & Dor & Thé et al., (2016) \\
\hline Pain Assessment Tool in Confused Elderly & IADIC & Dor & Saurin et al., (2013) \\
\hline Pain Catastrophizing Scale & PCS & Dor Lombar & Lopes et al., (2015) \\
\hline Pain Locus of Control Scale - Form C & PLOC-C & Dor & Pereira et al., (2011) \\
\hline Pain Response to Activity and Positioning Questionnaire & PRAP & Dor Lombar & De Carvalho et al., (2019) \\
\hline Parkinson's Disease Questionnaire & PDQ-39 & $\begin{array}{l}\text { Qualidade de Vida em } \\
\text { indivíduos com Doença de } \\
\text { Parkinson }\end{array}$ & Carod-Artal et al., (2007) \\
\hline Parkinson's Disease Sleep Scale & PDSS & Distúrbios do Sono & Margis et al., (2009) \\
\hline Patient-Generated Subjective Global Assessment & PG-SGA & Risco nutricional & Campos et al., (2012) \\
\hline Physical Activity Social Support Assessment Scale & ASAFA & $\begin{array}{l}\text { Apoio Social para Prática } \\
\text { de Atividade Física }\end{array}$ & Reis et al., (2011) \\
\hline Protocole Montréal d'Evaluation de la Communication - MEC & Protocolo & Distúrbios da Fala & Fonseca et al., (2008) \\
\hline Purpose in Life Scale & NI & Propósito de Vida & Ribeiro et al., (2018) \\
\hline Quality of Life and Swallowing Questionnaire & SWA-QOL & $\begin{array}{l}\text { Qualidade de Vida em } \\
\text { indivíduos com Parkinson }\end{array}$ & Diniz et al., (2018) \\
\hline Quality of Life Assessment Scale & $\begin{array}{l}\text { QOL; } \\
\text { QOL-AD }\end{array}$ & $\begin{array}{l}\text { Qualidade de Vida (em } \\
\text { indivíduos com Doença de } \\
\text { Alzheimer) }\end{array}$ & $\begin{array}{l}\text { Novelli et al., (2005); } \\
\text { Novelli et al., (2010) }\end{array}$ \\
\hline Questionnaire for the Evaluation of Informal Social Support for the Elderly & NI & Apoio Social & Guedes et al., (2018) \\
\hline Readiness for Hospital Discharge Scale & RHDS & $\begin{array}{l}\text { Preparo para Alta } \\
\text { Hospitalar }\end{array}$ & Siqueira et al., (2018) \\
\hline Relationship Scales Questionnaraire & RSQ & Apego & de Assis et al., (2019) \\
\hline Retirement Resources Inventory & RRI & $\begin{array}{l}\text { Recursos sobre Bem-Estar } \\
\text { na Aposentadoria }\end{array}$ & Amorim et al., (2019) \\
\hline
\end{tabular}




\begin{tabular}{|c|c|c|c|}
\hline Roland Morris Disability Questionnaire & RMDQ & Dor na Coluna & Sardá Jr et al., (2010) \\
\hline Rosenberg's Self-esteem Scale & RSES & Autoestima & Meurer et al., (2012) \\
\hline Sarcopenia Quality of Life & SarQol & $\begin{array}{l}\text { Qualidade de Vida em } \\
\text { idosos com Sarcopenia }\end{array}$ & Geenrik et al., (2019) \\
\hline Scale Pain Assessment in Advanced Dementia & PAINAD & Dor & Valera et al., (2014) \\
\hline Scales for Outcomes in Parkinson's Disease-Psychosocial Questionnaire & SCOPA-OS & $\begin{array}{l}\text { Funcionamento } \\
\text { Psicossocial }\end{array}$ & Carod-Artal et al., (2007) \\
\hline Screening for Voice Disorders in Older Adults & RAVI & Distúrbios de voz & $\begin{array}{l}\text { De Araujo Pernambuco et al., (2016); } \\
\text { Pernambuco et al., (2016) }\end{array}$ \\
\hline Self-Report Social Adjustment Scale & SAS-SR & Ajustamento Social & Gorenstein et al., (2002) \\
\hline Short Personal Experiences Questionnaire & SPEQ & Disfunção Sexual & Valadares et al., (2010) \\
\hline Short-Form Health Survey (Version 2) & $\begin{array}{l}\text { SF-36; } \\
\text { SF-12v2 }\end{array}$ & Qualidade de Vida & $\begin{array}{l}\text { Castro et al., (2014); } \\
\text { Damásio et al., (2015); } \\
\text { Laguardia et al., (2011); } \\
\text { Saccomann et al., (2010) }\end{array}$ \\
\hline Spiritual Health And Life-Orientation Measure & SHALOM & Bem-Estar Espiritual & Nunes et al., (2018) \\
\hline Successful Aging Scale & SAS & $\begin{array}{l}\text { Envelhecimento Bem- } \\
\text { Sucedido }\end{array}$ & Da Silva-Sauer et al., (2020) \\
\hline Temperament and Character Inventory - Revised & TCI-R & Temperamento & Gonçalves et al., (2010) \\
\hline UCLA Loneliness Scale & UCLA & Solidão & Kuznier, (2016) \\
\hline Utian Quality of Life & UQOL & $\begin{array}{l}\text { Qualidade de Vida no } \\
\text { Climatério }\end{array}$ & Lisboa et al., (2015) \\
\hline Utrecht Work Engagement Scale & $\begin{array}{l}\text { UWES-9; } \\
\text { UWES-17 }\end{array}$ & Engajamento no Trabalho & Vazquez et al., (2015) \\
\hline Vitor Quality of Life Scale for the Elderly & VITOR QLSE & Qualidade de vida & Da Silva et al., (2016) \\
\hline
\end{tabular}




\begin{tabular}{|c|c|c|c|}
\hline Voice Handicap Index & VHI & Autopercepção Vocal & Behlau et al., (2011) \\
\hline Vulnerable Elders Survey & VES-13 & Vulnerabilidade à Saúde & Silva et al., (2019) \\
\hline Western Aphasia Battery - Revised & WAB-R & Distúrbios de Linguagem & Neves et al., (2014) \\
\hline Wisconsin Brief Pain Questionnaire & WBPQ & Dor Crônica & Toledo et al., (2013) \\
\hline Women's Health Questionnaire & WHQ & $\begin{array}{l}\text { Qualidade de Vida no } \\
\text { Climatério }\end{array}$ & Silva Filho et al., (2005) \\
\hline Work Related Flow Inventory & WOLF & $\begin{array}{l}\text { Experiências Positivas e } \\
\text { Estado Mental }\end{array}$ & De Freitas et al., (2019) \\
\hline World Health Organization Quality of Life & $\begin{array}{l}\text { WHOQOL-BREF; } \\
\text { WHOQOL-OLD }\end{array}$ & Qualidade de Vida & $\begin{array}{l}\text { Casamali et al., (2019); } \\
\text { Casamali et al., (2019); } \\
\text { Castro et al., (2014); } \\
\text { Chachamovich et al., (2006); } \\
\text { Chachamovich et al., (2008); } \\
\text { Fleck et al., (2006) } \\
\text { Melo et al., (2018); } \\
\text { Silva et al., (2014) } \\
\text { Silva et al., (2019) }\end{array}$ \\
\hline
\end{tabular}

\section{NI - Não Informado}

Fonte: Aline Cristina da Silva, 2020 
Dos 74 instrumentos listados, 15 são dedicados a avaliar "qualidade de vida", sendo seis destinados a adultos e idosos no geral (CASAMALI et al., 2019; CASTRO et al., 2014; CHACHAMOVICH et al., 2006; CHACHAMOVICH et al., 2008; DAMÁSIO et al., 2015; DA SILVA et al., 2016; FLECK et al., 2006; LAGUARDIA et al., 2011; LIMA et al., 2014; NERI et al., 2018; NOVELLI et al., 2005; PASCHOAL et al., 2008; SACCOMANN et al., 2010; MELO et al., 2018; SILVA et al., 2014; SILVA et al., 2019;) e outros nove, são dedicados a avaliar qualidade de vida em populações específicas como, indivíduos com Doença de Parkinson e hemiplegia (CAROD-ARTAL et al., 2007; DINIZ et al., 2018; TEIXEIRASALMELA et al., 2004), indivíduos com Doença de Alzheimer (NOVELLI et al., 2010), mulheres/idosas na peri/menopausa e/ou climatério (LIMA et al., 2012; LISBOA et al., 2015; SILVA FILHO et al., 2005), idosos com insuficiência cardíaca (SACCOMANN et al., 2007) e idosos com sarcopenia (GEENRIK et al., 2019).

O instrumento mais conhecido e utilizado para esta variável é o WHOQOL, analisado em oito estudos desta análise (CASAMALI et al., 2019; CASTRO et al., 2014; CHACHAMOVICH et al., 2006; CHACHAMOVICH et al., 2008; FLECK et al., 2006; MELO et al., 2018; SILVA et al., 2014 e SILVA et al., 2019), apresentado em duas versões, WHOQOL-BREF e WHOQOL-OLD, reúne diferentes tipos de evidências de validade, com estudos de pontos de corte mais adequado, a depender da população analisada. Todos os estudos foram realizados com amostras exclusiva de idosos, com amplitude de idade relatada de 60 a 91 anos, sendo a maior amostra de 620 e a menor de 98 idosos, de pelo menos cinco Estados brasileiros. Além do WHOQOL, outro instrumento que se mostrou alvo de interesse dos pesquisadores, foi o SF-36, contemplado em quatro estudos (CASTRO et al., 2014; DAMÁSIO et al., 2015; LAGUARDIA et al., 2011 e SACCOMANN et al., 2010), sendo um deles com uma versão mais curta ainda SF-12v2, se propõem a medir qualidade de vida por meio de diferentes dimensões.

Vale destacar o estudo de Laguardia et al., (2011) que contou com uma amostra de 5.255 adultos e idosos, entre 40 e 64 anos e o estudo de Damásio et al., (2015) que contou com uma amostra pertencente a 17 Estados brasileiros. A dor foi uma variável que se mostrou de grande de interesse dos estudiosos para a população idosa, contemplada nesta análise em 12 instrumentos entre os estudos analisados (DE ABREU et al., 2008; DE CARVALHO et al., 2019; FERRARI et al., 2008; GAMBARO et al., 2009; LOPES et al., 2015; MOTTA et al., 2015; PEREIRA et al., 2011; SARDÁ JR et al., 2010; SAURIN et al., 2013; TEIXEIRA et al., 2020; THÉ et al., 2016; TOLEDO et al., 2013; VALERA et al., 2014). Foram analisados 
em amostras exclusivamente de idosos os instrumentos PAINAD, PACSLAC, GPM, PLOC-C e IADIC - que se propõem a medir dor; PRAP - dor lombar; MFPDI - dor no pé; e BBQ - dor aguda.

Os demais instrumentos, contemplaram também adultos na composição amostral, sendo destinados a medir WBPQ - dor crônica; FABQ-Brazil, PCS, RMDQ - dor na coluna/lombar. Sendo a qualidade de vida uma variável multidimensional, outros instrumentos foram identificados e contemplados nesta subcategoria a fim de apoiar a escolha entre instrumentos mais amplos e/ou específicos, dependendo da necessidade do aplicador. Foram identificados pelo menos outras 46 variáveis latente relacionadas à qualidade de vida, saúde e bem-estar, que dispõem de um instrumento específico para ser mensurada. É importante ter clareza de quem é a sua população e o que se pretende avaliar, só assim, será possível escolher adequadamente o instrumento que possa atender sua necessidade. A tabela 7, reúne na subcategoria Síndromes Geriátricas e Violência, um total de 12 instrumentos. Trata-se de temas distintos que foram agregados por terem um relevante ponto de convergência: a velhice e suas vulnerabilidades.

Tabela 7. Instrumentos para idosos brasileiros - Subcategoria: Síndromes Geriátricas e Violência, 2020

\begin{tabular}{|c|c|c|c|}
\hline Instrumento & Acrônimo & Variável latente & Autor; (Ano) \\
\hline \multirow{2}{*}{ Caregiver Abuse Screen } & \multirow{2}{*}{ CASE } & Negligência e Maus & Paixão et al., (2007); \\
\hline & & Tratos de Cuidadores & Reichenheim et al., (2009) \\
\hline \multirow{2}{*}{ Edmonton Frail Scale } & \multirow{2}{*}{ EFS } & \multirow{2}{*}{ Fragilidade } & \\
\hline & & & Fabrício-Wehbe et al., (2013) \\
\hline Frail Scale & NI & Fragilidade & Aprahamian et al., (2016) \\
\hline Geriatric Locomotive Function Scale & GLFS-25 & Síndrome Locomotora & Tavares et al., (2017) \\
\hline $\begin{array}{l}\text { Hwalek-Sengstock Elder Abuse } \\
\text { Screening Test }\end{array}$ & H-S/EAST & Violência Doméstica & Reichenheim et al., (2008) \\
\hline Kihon Checklist (Frailty Index) & $\mathrm{KCL}$ & Fragilidade & Sewo Sampaio et al., (2014) \\
\hline Rapid Geriatric Assessment & RGA & Síndromes Geriátricas & de Souza Orlandi et al., (2018) \\
\hline SARC-F Questionnaire & SARC-F & Sarcopenia & Barbosa-Silva et al., (2016) \\
\hline \multicolumn{4}{|l|}{ Structured Interwiew for the } \\
\hline $\begin{array}{l}\text { Diagnosis of Alzheimer's Type } \\
\text { Dementia, Multi-Infarct Dementia } \\
\text { and Dementia of other Aetiology }\end{array}$ & SIDAM & Síndromes Demenciais & Ventura et al., (2001) \\
\hline Targeted Geriatric Assessment & TaGA & Fragilidade & Aliberti et al., (2018) \\
\hline Tilburg Frailty Indicator & TFI & Fragilidade & $\begin{array}{l}\text { Santiago et al., (2013); } \\
\text { Santiago et al., (2018) }\end{array}$ \\
\hline
\end{tabular}




\begin{tabular}{|c|c|c|c|}
\hline $\begin{array}{l}\text { Vulnerability to Abuse Screening } \\
\text { Scale }\end{array}$ & VASS & Violência Doméstica & Dantas et al., (2017) \\
\hline
\end{tabular}

NI - Não Informado

Fonte: Aline Cristina da Silva, 2020

Em relação aos instrumentos de mensuração à abusos ou maus-tratos aos idosos, foram contemplados neste estudo, três diferentes, sendo: CASE (PAIXÃO et al., 2007; REICHENHEIM et al., 2009); H-S/EAST (REICHENHEIM et al., 2008) e VASS (DANTAS et al., 2017). Todos os estudos foram realizados exclusivamente com amostra de idosos, exceto o estudo de Paixão et al., (2007) pois se trata de um estudo metodológico, que contemplou apenas a tradução e adaptação transcultural do instrumento. Os outros nove instrumentos desta subcategoria, referem-se as chamadas síndromes geriátricas, ou seja, específicas e/ou típicas da idade, como a fragilidade, sarcopenia, demenciais e a locomotiva. Todos estes estudos foram realizados com amostras exclusivas de idosos, sem a participação de adultos. Aliberti et al., (2018), considerou idosos hospitalizados no estudo do instrumento TaGA, já o estudo de Ventura et al., (2001), considerou idosos com diagnóstico de demência, visto o interesse do instrumento SIDAM, destinado a esta característica.

\subsubsection{Propriedades Psicométricas}

Todos os estudos incluídos para análise trataram de evidências de validade. Logo, 86,07\% (204) relataram um ou mais tipo de evidências de validade, dentre elas, construto, conteúdo, face, divergente, convergente e discriminante; 59,92\% (142) dos estudos relataram evidências de confiabilidade; $28,27 \%$ (67) realizaram tradução e adaptação transcultural. Nesse cenário torna-se relevante outro aspecto, nos dois últimos Standards for Educational and Psychological Testing $(1999 ;$ 2014) já estabeleceram novos padrões e exigências para as etapas de validade, em um modelo que estabelece cinco tipos de evidências de validade: conteúdo, processo de resposta, estrutura interna (ex-construto), evidências com outras variáveis (excritério) e consequência do teste. Além disso estabelece que o processo de evidências de validade é um conceito unitário.

Interessante notar que desde 1999, a validade de face não faz parte mais das boas práticas e não há recomendação para sua aplicação, bem como divergente, convergente e discriminantes são procedimentos que compõem as evidências com outras variáveis. Ainda mais relevante é o conceito de confiabilidade que diz respeito a garantia de que o instrumento realmente mensura a variável latente a que se destina e de forma precisa. O que não deve ser 
confundido com as multiplicidades de técnicas de confiabilidade que podem ser aplicadas em vários momentos na busca de evidências. Esses apontamentos tomam vulto por um descolamento das boas práticas psicométricas e suas recomendações e do que se tem praticado (BORSBOOM, 2006; KANE, 2015; NEWTON; SHAW, 2014). Os procedimentos adotados e recomentados na busca de evidências de validade não é um sistema dicotômico (validado ou não-validado), como aponta Zumbo (2007), e nem uma atividade de um tempo único (SHEPARD, 1993), pois há necessidade periódica de revisita das propriedades dos instrumentos quanto ao seu contexto de aplicação, população e a cultura atual. A busca realizada não encontrou estudo de revisita as propriedades do instrumento.

\subsection{CONCLUSÕES E RECOMENDAÇÕES}

É notável o crescimento das pesquisas/estudos psicométricos relacionados à população idosa. Reunir e expor todos estes 192 instrumentos, reflete a diversidade de temas a serem pesquisados/enfrentados não só pela Gerontologia e Geriatria, mas por todas as áreas/campos da saúde e social. O ser humano é complexo e a cada dia revela uma nova faceta a ser analisada em suas relações com si próprio, com o outro e com o ambiente em que vive. Logo, haverá sempre uma nova variável latente a ser explorada, ou mesmo uma já conhecida, que se manifestará por comportamentos distintos, mediante as diferentes épocas geracionais e acordos sociais. A pesquisa nacional ainda precisa ser mais cautelosa e rigorosa metodologicamente, cumprindo todas as etapas recomendadas na literatura de acordo com cada desenho de pesquisa definido, mas principalmente, zelar pela qualidade à quantidade, garantido uma escrita clara, objetiva, passível de compreensão e reprodução, bem como, aplicação do estado da arte contemporâneo das evidências de validade e que os relatórios sejam completos (AERA; APA; NCME, 2014).

Este estudo não teve como objetivo esgotar as alternativas de instrumentos disponíveis para esta população, mas sim possibilitar uma visão mais ampliada, reunindo em uma única fonte, evidências que possam gerar reflexão e agregar para a pesquisa e a prática de todos os interessados pela temática. A Gerontologia é um campo multidisciplinar crescente e necessita de especialistas para responder os diversos dilemas que surgem todos os dias frente a ascensão cronológica da vida humana e o mundo moderno. Quanto as limitações deste estudo, não foram incluídas buscas manuais ou em literatura cinza de outros instrumentos conhecidos, como recomendado nos manuais para este método de pesquisa. Além disso, não cabe no escopo dos procedimentos metodológicos adotado nesse artigo, a análises pormenorizada dos 
procedimentos, técnicas, qualidade dos indicadores adotados nos artigos que encontramos. Esse é o desafio futuro.

\subsection{CONFLITOS E RECONHECIMENTOS}

Esta scoping review é parte integrante da Dissertação de Mestrado da autora. Não há conflitos de interesse por parte de nenhum pesquisador que participou deste estudo como: autor, orientador, revisores ou outros, em relação a nenhuma das etapas realizadas, bem como o desfecho referido. Este estudo não contou com nenhum financiamento, parcial ou integral, para sua realização. Desde a idealização do projeto de pesquisa até a versão final, apresentada em defesa oficial para a banca examinadora convidada.

\section{REFERÊNCIAS}

AMERICAN EDUCATIONAL RESEARCH ASSOCIATION (AERA); AMERICAN PSYCHOLOGICAL ASSOCIATION (APA); NATIONAL COUNCIL ON MEASUREMENT IN EDUCATION (NCME). Standards for educational and psychological testing. Washington, DC: Author, 1999.

AMERICAN EDUCATIONAL RESEARCH ASSOCIATION (AERA); AMERICAN PSYCHOLOGICAL ASSOCIATION (APA); NATIONAL COUNCIL ON MEASUREMENT IN EDUCATION (NCME). Standards for educational and psychological testing. Washington, DC: Author, 2014.

APÓSTOLO, J. L. A. Instrumentos para Avaliação em Geriatria (Geriatric Instruments). ESENFC, 2012.2 Disponível em: https://web.esenfc.pt/v02/include/download.php?id_ficheiro=20538\&codigo=688697509.

Acesso em: 29 jan. 2020.

BALTES, M. M.; CARSTENSEN, L. L. The process of successful ageing. Ageing and Society, Ageing and Society, v. 16, n. 4, p. 397-422, 1996. DOI: 10.1017/S0144686X00003603. Disponível em: https://www.cambridge.org/core/journals/ageing-and-society/article/processof-successful-ageing/C1E6F81ADC2C4D91484A8819C7BED061. Acesso em: 29 jan. 2020.

BORSBOOM, D. The attack of the psychometricians. Psychometrika, v. 71, n. 3, p. 425-440, 2006. DOI: 10.1007/s11336-006-1447-6. Disponível em: https://PubMed.ncbi.nlm.nih.gov/19946599/. Acesso em: 29 jan. 2020.

KANE, M. T. Explicating validity. Assessment in Education: Principles, Policy \& Practice, v. 23, n. 2, p. 198-211, 2016. DOI: https://doi.org/10.1080/0969594X.2015.1060192 Disponível em: https://www.tandfonline.com/doi/abs/10.1080/0969594X.2015.1060192. Acesso em: 29 jan. 2020. 
KUANG, M., et al. Taking Diversity into Account: Assessment of Older Adults. In: LEONG, F. T. et al. (Eds.). The ITC International Handbook of Testing and Assessment. Oxford: Oxford University Press, 2016. p. 290 - 299.

MEDEIROS, P. A. et al. Instrumentos desenvolvidos para o gerenciamento e cuidado de idosos em instituições de longa permanência: uma revisão sistemática. Ciência \& Saúde Coletiv, v. 21, n. 11, p. 3597-3610, 2016. DOI: https://doi.org/10.1590/1413-812320152111.09912015. Disponível em: https://www.scielo.br/scielo.php?pid=S1413-81232016001103597. Acesso em: 29 jan. 2020.

NEWTON, P. E., SHAW, S. D. Validity in educational and psychological assessment. Londres: SAGE Publications Ltd., 2014. ISBN:9781446288856.

PARANÁ. Secretaria de Estado da Saúde do Paraná. Superintendência de Atenção à Saúde. Avaliação multidimensional do idoso / SAS. Curitiba, 2017. Disponível em: https://www.saude.pr.gov.br/sites/default/arquivos_restritos/files/documento/202004/avaliacaomultiddoidoso_2018_atualiz.pdf. Acesso em: 29 jan. 2021.

PEDREIRA, R. B. S. et al. Validade de conteúdo do Instrumento de Avaliação da Saúde do Idoso. Einstein (São Paulo), São Paulo, v. 14, n. 2, p. 158-177, 2016. DOI: https://doi.org/10.1590/S1679-45082016AO3455. Disponível em: https://www.scielo.br/scielo.php?pid=S1679-45082016000200009. Acesso em: 29 jan. 2021.

PETERS, M. D. J. et al. Capítulo 11: Revisões do escopo (versão 2020). In: AROMATARIS, E.; MUNN, Z. (Eds). Manual da JBI para síntese de evidências. (Online) JBI, 2020. DOI: https://doi.org/10.46658/JBIMES-20-12. Disponível em: https://synthesismanual.jbi.global. Acesso em: 29 jan. 2021.

RYAN, R., SYNNOT, A., PRICTOR, M., HILL, S. Cochrane Consumers and Communication Group Data extraction template for included studies. CCCG. Disponível: http://cccrg.cochrane.org/author-resources. La Trobe University, Melbourne, 2016. Acesso em: 28 set 2020 .

SHEPARD, L. A. Evaluating test validity. In: Review of Research in Education, 1993. v. 19, n. 1, p. 405-450. DOI: https://doi.org/10.3102/0091732X019001405.

TRICCO, A. C. et al. PRISMA Extension for Scoping Reviews (PRISMA-ScR): Checklist and Explanation. Annals of Internal Medicine, 2018. DOI: https://doi.org/10.7326/M18-0850. Disponível em: https://www.acpjournals.org/doi/pdf/10.7326/M18-0850. Acesso em: 29 jan. 2021.

VERAS, R. P. Guia dos Instrumentos de Avaliação Geriátrica [Recurso Eletrônico] / Renato Peixoto Veras. - Rio de Janeiro: Unati/UERJ, 2019. 20 f.: il. ISBN: 978-85-87897-26-8. Disponível em: http://www.unatiuerj.com.br/Guia\%20dos\%20instrumentos\%20Avaliacao\%20Geriatrica.pdf. Acesso em: 29 jan. 2021.

ZUMBO, B. D. Validity: Foundational issues and statistical methodology. In: RAO, C. R.; SINHARAY, S. (Eds.), Psychometrics. Amsterdam: Elsevier Science, 2007. p. 45-79. DOI: https://doi.org/10.1016/S0169-7161(06)26003-6. 


\section{*Referências dos estudos incluídos na revisão}

AHMED, T.; VAFAEI, A.; BELANGER, E.; PHILLIPS, S. P.; ZUNZUNEGUI, M. V. Bem Sex Role Inventory Validation in the International Mobility in Aging Study. Can J Aging, v. 35, n. 3, p. 348-60, 2016. DOI: 10.1017/S0714980816000404. Disponível em: https://PubMed.ncbi.nlm.nih.gov/27477107/. Acesso em: 18 jan. 2021.

ALIBERTI, M. J. R. et al. Targeted Geriatric Assessment for Fast-Paced Healthcare Settings: Development, Validity, and Reliability. Journal of the American Geriatrics Society, v. 66, n. 4 , p. 748-754, 2018. DOI:10.1111/jgs.15303. Disponível em: https://PubMed.ncbi.nlm.nih.gov/29473941/. Acesso em: 18 jan. 2021.

ALMEIDA, C. B. L. et al. Pain-induced depression in the elderly: Validation of psychometric properties of the Brazilian version of the "Geriatric Emotional Assessment of Pain" - GEAP-b. Rev. Assoc. Med. Bras., São Paulo, v. 63, n. 9, p. 741-746, 2017. DOI: http://dx.doi.org/10.1590/1806-9282.63.09.741. Disponível em: http://www.scielo.br/scielo.php?script=sci_arttext\&pid=S0104-42302017000900741. Acesso em: 18 jan. 2021.

ALMEIDA, M. H. M. et al . Reliability of the Instrument for Classifying Elderly People's Capacity for Self-care. Rev. Saúde Pública, São Paulo, v. 42, n. 2, p. 317-323, 2008. DOI: https://doi.org/10.1590/S0034-89102008005000003. Disponível em: http://www.scielo.br/scielo.php?script=sci_arttext\&pid=S0034-89102008000200018. Acesso em 18 jan. 2021.

ALVARENGA, M. R.; OLIVEIRA, M. A. C.; FACCENDA, O. Depressive symptoms in the elderly: analysis of the items of the Geriatric Depression Scale. Acta paul. enferm., São Paulo, v. 25, n. 4, p. 497-503, 2012. DOI: http://dx.doi.org/10.1590/S0103-21002012000400003. Disponível em: http://www.scielo.br/scielo.php?script=sci_arttext\&pid=S010321002012000400003. Acesso em: 18 jan. 2021.

AMORIM, S. M.; FRANÇA, L. H. F. P. Validity Evidence of the Retirement Resources Inventory. The Spanish journal of psychology, v. 22, p. E23, 2019. DOI: https://doi.org/10.1017/sjp.2019.23.

Disponível

em: https://www.cambridge.org/core/journals/spanish-journal-of-psychology/article/abs/validityevidence-of-the-retirement-resources-inventory/2B104BA13DDAA2D9448719C0F40525D9. Acesso em: 18 jan. 2021.

APOLINARIO, D. et al. Estimating premorbid cognitive abilities in low-educated populations. PloS one, v. 8, n. 3, p. e60084, 2013. DOI:10.1371/journal.pone.0060084. Disponível em: https://PubMed.ncbi.nlm.nih.gov/23555894/. Acesso em: 18 jan. 2021.

APRAHAMIAN, I. et al. Feasibility and Factor Structure of the FRAIL Scale in Older Adults. Journal of the American Medical Directors Association, v. 18, n. 4, p. 367.e11-367.e18, 2017. DOI:10.1016/j.jamda.2016.12.067. Disponível em: https://PubMed.ncbi.nlm.nih.gov/28214239/. Acesso em: 18 jan. 2021.

ASSIS, L. O. et al. Psychometric properties of the Brazilian version of Pfeffer's Functional Activities Questionnaire. Frontiers in aging neuroscience, v. 6 p.255, 2014. 
DOI:10.3389/fnagi.2014.00255. Disponível em: https://PubMed.ncbi.nlm.nih.gov/25309432/. Acesso em: 18 jan. 2021.

ATALAIA-SILVA, K. C.; LOURENCO, R. A. Tradução, adaptação e validação de construto do Teste do Relógio aplicado entre idosos no Brasil. Rev. Saúde Pública, São Paulo, v. 42, n. 5, p. 930-937, 2008. DOI: http://dx.doi.org/10.1590/S0034-89102008000500020. Disponível em: http://www.scielo.br/scielo.php?script=sci_arttext\&pid=S0034-89102008000500020. Acesso em: 18 jan. 2021.

BAGGIO, J. A. O. et al. Validation of the Brazilian version of the Clinical Gait and Balance Scale and comparison with the Berg Balance Scale. Arq. Neuro-Psiquiatr., São Paulo , v. 71, n. 9A, p. 621-626, 2013. DOI: https://doi.org/10.1590/0004-282X20130107. Disponível em: http://www.scielo.br/scielo.php?script=sci_arttext\&pid=S0004-282X2013000900621. Acesso em: 18 jan. 2021.

BAGGIO, J. A. O. et al. Validity of the Brazilian version of the freezing of gait questionnaire. Arq. Neuro-Psiquiatr., São Paulo, v. 70, n. 8, p. 599-603, 2012. DOI: https://doi.org/10.1590/S0004-282X2012000800008. Disponível em: http://www.scielo.br/scielo.php?script=sci_arttext\&pid=S0004-282X2012000800008. Acesso em: 18 jan. 2021.

BAHIA, V. S. et al. Functional disability in Alzheimer disease: a validation study of the Brazilian version of the Disability Assessment for Dementia (DAD-Br). Alzheimer Disease \& Associated Disorders, v. 24, n. 3, p. 291-295, 2010. DOI: 10.1097/WAD.0b013e3181cfc878. Disponível em: http://repositorio.unifesp.br/handle/11600/32699. Acesso em: 18 jan. 2021.

BANHATO, E. F. C. et al. Criterion validity of a Wechsler-III scale short form in a sample of Brazilian Elderly. Dement. neuropsychol., São Paulo, v. 4, n. 3, p. 207-213, 2010. DOI: https://doi.org/10.1590/S1980-57642010DN40300009. Disponível em: http://www.scielo.br/scielo.php?script=sci_arttext\&pid=S1980-57642010000300207. Acesso em: 18 jan. 2021.

BARBOSA-SILVA, T. G. et al. Enhancing SARC-F: Improving Sarcopenia Screening in the Clinical Practice. Journal of the American Medical Directors Association, v. 17, n. 12, p. 1136-1141, 2016. DOI:10.1016/j.jamda.2016.08.004. Disponível em: https://PubMed.ncbi.nlm.nih.gov/27650212/. Acesso em: 18 jan. 2021.

BASTONE, A. C. et al. Validation of the human activity profile questionnaire as a measure of physical activity levels in older community-dwelling women. Journal of aging and physical activity, v. 22, n. 3, p. 348-56, 2014. DOI:10.1123/japa.2013-0006. Disponível em: https://PubMed.ncbi.nlm.nih.gov/23917084/. Acesso em: 18 jan. 2021.

BATISTONI, S. S. T.; NERI, A. L.; CUPERTINO, A. P. F. B. Validade da escala de depressão do Center for Epidemiological Studies entre idosos brasileiros. Rev. Saúde Pública, São Paulo, v. 41, n. 4, p. 598-605, 2007. DOI: https://doi.org/10.1590/S0034-89102007000400014. Disponível em: http://www.scielo.br/scielo.php?script=sci_arttext\&pid=S003489102007000400014. Acesso em: 18 jan. 2021.

BEHLAU, M. et al. Cross-cultural adaptation and validation of the voice handicap index into Brazilian Portuguese. Journal of voice: official journal of the Voice Foundation, v. 25,n. 3, 
p. 354-359, 2011. DOI:10.1016/j.jvoice.2009.09.007. Disponível em: https://PubMed.ncbi.nlm.nih.gov/20434874/. Acesso em: 18 jan. 2021.

BERTOLUCCI, P. H. F. et al. Applicability of the CERAD neuropsychological battery to Brazilian elderly. Arq. Neuro-Psiquiatr., São Paulo, v. 59, n. 3A, p. 532-536, 2001. DOI: https://doi.org/10.1590/S0004-282X2001000400009. Disponível em: http://www.scielo.br/scielo.php?script=sci_arttext\&pid=S0004-282X2001000400009. Acesso em: 18 jan. 2021.

BLAY, S. L.; RAMOS, L. R.; MARI, J. J. Validity of a Brazilian version of the Older Americans Resources and Services (OARS) mental health screening questionnaire. Journal Of The American Geriatrics Society, São Paulo, v. 36, n. 8, p. 687-692, 1988. DOI: http://dx.doi.org/10.1111/j.1532-5415.1988.tb07169.x. Disponível em: https://repositorio.unifesp.br/handle/11600/44731. Acesso em: 23 jan. 2021.

BORGES, M. K.; JACINTO, A. F.; CITERO, V. A. Cross-cultural adaptation of the "Australian National University Alzheimer's Disease Risk Index" for the Brazilian population. Dement. neuropsychol., São Paulo, v. 11, n. 2, p. 162-175, 2017. DOI: http://dx.doi.org/10.1590/198057642016dn11-020009. Disponível em: http://www.scielo.br/scielo.php?script=sci_arttext\&pid=S1980-57642017000200162. Acesso em: 23 jan. 2021.

BRUM, P. S. et al. Categorization Working Memory Span Task: Validation study of two Brazilian alternate versions. International Journal of Geriatric Psychiatry, v. 33, n. 4, p. 652-657, 2018. DOI: https://doi.org/10.1002/gps.4836. Disponível em: https://bv.fapesp.br/pt/publicacao/141925/categorization-working-memory-span-taskvalidation-study-of. Acesso em: 23 jan. 2021.

CALDAS, V. V. A. et al. Translation, cultural adaptation and psychometric evaluation of the Leganés cognitive test in a low educated elderly Brazilian population. Arq. Neuro-Psiquiatr., São Paulo, v. 70, n. 1, p. 22-27, 2012. DOI: https://doi.org/10.1590/S0004282X2012000100006. Disponível em: http://www.scielo.br/scielo.php?script=sci_arttext\&pid=S0004-282X2012000100006. Acesso em: 23 jan. 2021.

CAMARGOS, F. F. O. et al. Adaptação transcultural e avaliação das propriedades psicométricas da Falls Efficacy Scale - International em idosos Brasileiros (FES-I-BRASIL). Rev. bras. fisioter., São Carlos, v. 14, n. 3, p. 237-243, 2010. DOI: https://doi.org/10.1590/S1413-35552010000300010. Disponível em: http://www.scielo.br/scielo.php?script=sci_arttext\&pid=S1413-35552010000300010. Acesso em: 23 jan. 2021.

CAMOZZATO, A. L. et al. Reliability of the Brazilian Portuguese version of the Neuropsychiatric Inventory (NPI) for patients with Alzheimer's disease and their caregivers. International Psychogeriatrics, v. 20, n. 2, p. 383-393, 2008. DOI: doi:10.1017/S1041610207006254. Disponível em: https://PubMed.ncbi.nlm.nih.gov/18257965/. Acesso em: 23 jan. 2021.

CAMPOS, J. A. D. B.; PRADO, C. D. Cross-cultural adaptation of the Portuguese version of the Patient-Generated Subjective Global Assessment. Nutr Hosp, v. 27, n. 2, p. 583-9, 2012. 
DOI: $\quad$ 10.1590/S0212-16112012000200035.

Disponível

em:

https://PubMed.ncbi.nlm.nih.gov/22732987/. Acesso em: 23 jan. 2021.

CAROD-ARTAL, F. J. et al. Independent validation of SCOPA-psychosocial and metric properties of the PDQ-39 Brazilian version. Mov Disord, v. 22, n. 1, p. 91-8, 2007. DOI: 10.1002/mds.21216. Disponível em: https://PubMed.ncbi.nlm.nih.gov/17094102/. Acesso em: 23 jan. 2021.

CAROD-ARTAL, F. J. et al. Psychometric properties of the SCOPA-AUT Brazilian Portuguese version. Movement Disorders, v. 25, n. 2, p. 205-212, 2010. DOI: 10.1002/mds.22882. Disponível em: https://PubMed.ncbi.nlm.nih.gov/19938162/. Acesso em: 23 jan. 2021.

CASAMALI, F. F. C. et al. Accordance and reproducibility of the electronic version of the WHOQOL-BREF and WHOQOL-OLD questionnaires. Exp. Gerontol., v. 125, 20192019. DOI: 10.1016/j.exger.2019.110683. Disponível em: https://PubMed.ncbi.nlm.nih.gov/31398444/. Acesso em: 23 jan. 2021.

CASTELO, M. S. et al. Validity of the Brazilian version of the Geriatric Depression Scale (GDS) among primary care patients. International Psychogeriatrics, v. 22, n. 1, p. 109-13, 2010. DOI: doi:10.1017/S1041610209991219. Disponível em: https://www.cambridge.org/core/journals/international-psychogeriatrics/article/abs/validityof-the-brazilian-version-of-the-geriatric-depression-scale-gds-among-primary-carepatients/A6ED434968E1A65899C3DADDF963DE44. Acesso em: 23 jan. 2021.

CASTRO, P. C.; DRIUSSO, P.; OISHI, J. Convergent validity between SF-36 and WHOQOLBREF in older adults. Rev. Saúde Pública, São Paulo, v. 48, n. 1, p. 63-67, 2014. DOI: https://doi.org/10.1590/S0034-8910.2014048004783. Disponível em: http://www.scielo.br/scielo.php?script=sci_arttext\&pid=S0034-89102014000100063. Acesso em: 23 jan. 2021.

CASTRO-COSTA, E. et al. Association of cognitive impairment, activity limitation with latent traits in the GHQ-12 in the older elderly. The Bambui Health and Aging Study (BHAS). Aging Clin Exp Res, v. 20, n. 6, p. 562-8, 2008. DOI: 10.1007/BF03324885. Disponível em: https://PubMed.ncbi.nlm.nih.gov/19179841/. Acesso em: 23 jan. 2021.

CASTRO-COSTA, E. et al. Construct validity of the mini mental state examination across time in a sample with low-education levels: 10-year follow-up of the Bambuí Cohort Study of Ageing. Int J Geriatr Psychiatry., v. 29, n. 12, p. 1294-303, 2014. DOI: 10.1002/gps.4113. Disponível em: https://www.arca.fiocruz.br/handle/icict/11105. Acesso em: 23 jan. 2021.

CASTRO-COSTA, E. et al. Dimensions underlying the mini-mental state examination in a sample with low-education levels: the Bambuí health and aging study. Am J Geriatr Psychiatry, v. 17, n. 10, p. 863-872, 2009. DOI: 10.1097/JGP.0b013e3181ab8b4d. Disponível em: https://PubMed.ncbi.nlm.nih.gov/19910875/. Acesso em: 23 jan. 2021.

CECATO, J. F. et al. A subtest analysis of the Montreal cognitive assessment (MoCA): which subtests can best discriminate between healthy controls, mild cognitive impairment and Alzheimer's disease? International psychogeriatrics, v. 28, n. 5, p. 825-832, 2016. DOI: 
10.1017/S1041610215001982. Disponível em: https://PubMed.ncbi.nlm.nih.gov/26620850/. Acesso em: 23 jan. 2021.

CECATO, J. F. et al. Clock drawing test in elderly individuals with different education levels: correlation with clinical dementia rating. Am J Alzheimers Dis Other Demen, v. 27, n. 8, p. 620-624, 2012. DOI: 10.1177/1533317512463954 Disponível em: https://PubMed.ncbi.nlm.nih.gov/23118201/. Acesso em: 23 jan. 2021.

CÉSAR, K. G. et al. Addenbrooke's cognitive examination-revised: normative and accuracy data for seniors with heterogeneous educational level in Brazil. International Psychogeriatrics, v. 29, n. 8, p. 1345-1353, 2017. DOI: 10.1017/S1041610217000734. Disponível em: https://PubMed.ncbi.nlm.nih.gov/28511735/. Acesso em: 23 jan. 2021.

CHACHAMOVICH, E. et al. Assessment of the psychometric performance of the WHOQOLBREF instrument in a sample of Brazilian older adults. International psychogeriatrics, v. 19, n. 4, p. 635-646, 2007. DOI: 10.1017/S1041610206003619. Disponível em: https://PubMed.ncbi.nlm.nih.gov/16870036/. Acesso em: 23 jan. 2021.

CHACHAMOVICH, E. et al. Brazilian WHOQOL-OLD Module version: a Rasch analysis of a new instrument. Rev Saude Publica, v. 42, n. 2, p. 308-316, 2008. DOI: https://doi.org/10.1590/S0034-89102008000200017. Disponível em: http://www.scielo.br/scielo.php?script=sci_arttext\&pid=S0034-89102008000200017. Acesso em: 23 jan. 2021.

CHACHAMOVICH, E. et al. Development and validation of the Brazilian version of the Attitudes to Aging Questionnaire (AAQ): An example of merging classical psychometric theory and the Rasch measurement model. Health \& Quality of Life Outcomes, v. 6, n. 5, 2008. DOI: https://doi.org/10.1037/t61721-000. Disponível em: https://psycnet.apa.org/record/2017-25657-001. Acesso em: 23 jan. 2021.

CHACHAMOVICH, E. et al. Is Geriatric Depression Scale-15 a suitable instrument for measuring depression in Brazil? Results of a Rasch analysis. Psychology, Health \& Medicine, v. 15, n. 5, p. 596-606, 2010. DOI: 10.1080/13548506.2010.487108. Disponível em: https://PubMed.ncbi.nlm.nih.gov/20835969/\#: :text=Data\%20were\%20analyzed\%20by\%20t he,suitable\%20in\%20a\%20Brazilian\%20sample. Acesso em: 23 jan. 2021.

CHAGAS, M. H. N. et al. Validation and internal consistency of Patient Health Questionnaire9 for major depression in Parkinson's disease. Age and ageing, v. 42, n. 5, p. 645-649, 2013. DOI: 10.1093/ageing/aft065. Disponível em: https://PubMed.ncbi.nlm.nih.gov/23761457/. Acesso em: 23 jan. 2021.

CHAGAS, M. H. N. et al. Validity of the PHQ-2 for the screening of major depression in Parkinson's disease: Two questions and one important answer. Aging \& Mental Health, v. 15, n. 7, p. 838-843, 2011. DOI: 10.1080/13607863.2011.569482. Disponível em: https://PubMed.ncbi.nlm.nih.gov/21562987/. Acesso em: 23 jan. 2021.

CHINI, L. T.; PEREIRA, D. S.; NUNES, A. A. Validation of the Fall Risk Tracking Tool (FRRISque) in elderly community dwellers. Ciênc. saúde coletiva, Rio de Janeiro, v. 24, n. 8, p. 2845-2858, 2019. DOI: http://dx.doi.org/10.1590/1413-81232018248.28962017. Disponível 
em:

https://www.scielo.br/scielo.php?pid=S1413-

81232019000802845\&script=sci_arttext\&tlng=en. Acesso em: 23 jan. 2021 .

CINTRA, F. C. M. C. et al. Functional decline in the elderly with MCI: Cultural adaptation of the ADCS-ADL scale. Revista da Associacao Medica Brasileira (1992), v. 63, n. 7, p. 590599, 2017. DOI: 10.1590/1806-9282.63.07.590. Disponível em: https://PubMed.ncbi.nlm.nih.gov/28977084/. Acesso em: 23 jan. 2021.

CORREIA, C. C. et al.. AD8-Brazil: Cross-cultural validation of the ascertaining dementia interview in portuguese. J. Alzheimer's Dis., v. 27, n. 1, p. 177-185, 2011. DOI: 10.3233/JAD2011-100915. Disponível em: https://PubMed.ncbi.nlm.nih.gov/21799253/. Acesso em: 23 jan. 2021.

COSTA, E. et al. Agreement between the Geriatric Depression Scale and the General Health Questionnaire in a population-based elderly cohort: the Bambuí Health \& Ageing Study (BHAS). Clinical Gerontologist, v. 26, n. 3, p. 69-82, 2003. DOI: https://doi.org/10.1300/J018v26n03_06. Disponível em: https://www.tandfonline.com/doi/abs/10.1300/J018v26n03_06. Acesso em: 23 jan. 2021.

COSTA, E. et al. Is the GDS-30 better than the GHQ-12 for screening depression in elderly people in the community? The Bambui Health Aging Study (BHAS). International Psychogeriatrics, v. 18, n. 3, p. 493-503, 2006. DOI: 10.1017/S1041610205002954. Disponível em: https://PubMed.ncbi.nlm.nih.gov/16478572/. Acesso em: 23 jan. 2021.

CURCIO, C. L. et al. Life-Space Assessment scale to assess mobility: validation in Latin American older women and men. Aging clinical and experimental research, v. 25, n. 5, p. 553-560, 2013. DOI: 10.1007/s40520-013-0121-y. Disponível em: https://PubMed.ncbi.nlm.nih.gov/23949973/. Acesso em: 23 jan. 2021.

DA SILVA, M. J. et al. Analysis of psychometric properties of family APGAR with elderly in northeast Brazil. Esc. Anna Nery, Rio de Janeiro, v. 18, n. 3, p. 527-532, 2014. DOI: http://dx.doi.org/10.5935/1414-8145.20140075. Disponível em: http://www.scielo.br/scielo.php?script=sci_arttext\&pid=S1414-81452014000300527. Acesso em: 23 jan. 2021.

DA SILVA, B. A. et al. Assessing Timed Up and Go in Parkinson's disease: Reliability and validity of Timed Up and Go Assessment of biomechanical strategies. Journal of rehabilitation medicine, v. 49, n. 9, p. 723-731, 2017. DOI:10.2340/16501977-2254. Disponível em: https://PubMed.ncbi.nlm.nih.gov/28951938/. Acesso em: 23 jan. 2021.

DA SILVA, J. V.; BAPTISTA, M. N. Vitor Quality of Life Scale for the Elderly: evidence of validity and reliability. SpringerPlus, v. 5, n. 1 p. 1450, 2016. DOI:10.1186/s40064-016-31304. Disponível em: https://PubMed.ncbi.nlm.nih.gov/27652026/. Acesso em: 23 jan. 2021.

DA SILVA-SAUER, L. et al. Cross-cultural adaptation and psychometric properties of the Brazilian Portuguese version of successful aging scale in community-dwelling older adults. Journal of community psychology, v. 48, n. 6, p. 1840-1852, 2020. DOI: https://doi.org/10.1002/jcop.22374. Disponível em: https://onlinelibrary.wiley.com/doi/abs/10.1002/jcop.22374. Acesso em: 23 jan. 2021. 
DAMASCENO, A. et al. Validation of the Brazilian version of mini-test CASI-S. Arq. NeuroPsiquiatr., São Paulo, v. 63, n. 2b, p. 416-421, 2005. Doi: http://dx.doi.org/10.1590/S0004282X2005000300010.

Disponível

em: http://www.scielo.br/scielo.php?script=sci_arttext\&pid=S0004-282X2005000300010. Acesso em: 23 jan. 2021.

DAMASIO, B. F.; BORSA, J. C.; KOLLER, S. H. Adaptation and Psychometric Properties of the Brazilian Version of the Five-item Mental Health Index (MHI-5). Psicol. Reflex. Crit., Porto Alegre, v. 27, n. 2, p. 323-330, 2014. DOI: http://dx.doi.org/10.1590/16787153.201427213.

http://www.scielo.br/scielo.php?script=sci_arttext\&pid=S0102-79722014000200323. Acesso em: 23 jan. 2021.

DAMÁSIO, B. F. et al. Is the General Self-Efficacy Scale a Reliable Measure to be used in Cross-Cultural Studies? Results from Brazil, Germany and Colombia. The Spanish journal of psychology, v. 19, n. 29, 2016, DOI:10.1017/sjp.2016.30. Disponível em: https://PubMed.ncbi.nlm.nih.gov/27225231/. Acesso em: 23 jan. 2021.

DAMÁSIO, B. F.; KOLLER, S. H. Meaning in Life Questionnaire: Adaptation process and psychometric properties of the Brazilian version. Revista Latinoamericana de Psicología, v. 47, n. 3, p. 185-195, 2015. DOI: https://doi.org/10.1016/j.rlp.2015.06.004. Disponível em: http://www.sciencedirect.com/science/article/pii/S0120053415000163. Acesso em: 23 jan. 2021.

DAMASIO, B. F.; ANDRADE, T. F.; KOLLER, S. H. Psychometric Properties of the Brazilian 12-Item Short-Form Health Survey Version 2 (SF-12v2). Paidéia (Ribeirão Preto), Ribeirão Preto, v. 25, n. 60, p. 29-37, 2015. DOI: https://doi.org/10.1590/1982-43272560201505. Disponível em: http://www.scielo.br/scielo.php?script=sci_arttext\&pid=S0103863X2015000100029. Acesso em: 23 jan. 2021.

DANTAS, R. B.; OLIVEIRA, G. L.; SILVEIRA, A. M. Psychometric properties of the Vulnerability to Abuse Screening Scale for screening abuse of older adults. Rev. Saúde Pública, São Paulo, v. 51, n. 31, 2017. DOI: http://dx.doi.org/10.1590/s15188787.2017051006839. Disponível em: http://www.scielo.br/scielo.php?script=sci_arttext\&pid=S0034-89102017000100224. Acesso em: 23 jan. 2021.

DE ABREU, A. M. et al. Versão brasileira do Fear Avoidance Beliefs Questionnaire. Cad. Saúde Pública, Rio de Janeiro, v. 24, n. 3, p. 615-623, 2008. DOI: https://doi.org/10.1590/S0102-311X2008000300015. Disponível em: http://www.scielo.br/scielo.php?script=sci_arttext\&pid=S0102-311X2008000300015. Acesso em: 23 jan. 2021.

DE ALMEIDA, M. H. M. et al. Reliability and Validity of the Brazilian Version of the LateLife Function and Disability Instrument. American Journal of Occupational Therapy, v. 70, n. 2, p. p1-p8, 2016. DOI: 10.5014/ajot.2016.017624. Disponível em: https://PubMed.ncbi.nlm.nih.gov/26943117/. Acesso em: 24 jan. 2021.

DE ARAUJO, N. B. et al. Diagnosing dementia in lower educated older persons: validation of a Brazilian Portuguese version of the Rowland Universal Dementia Assessment Scale 
(RUDAS). Rev. Bras. Psiquiatr., São Paulo, v. 40, n. 3, p. 264-269, 2018. DOI: http://dx.doi.org/10.1590/1516-4446-2017-2284.

Disponível

em: https://www.scielo.br/scielo.php?pid=S1516-44462018000300264\&script=sci_abstract. Acesso em: 24 jan. 2021.

DE ARAÚJO PERNAMBUCO, L. et al. Screening for Voice Disorders in Older Adults (Rastreamento de Alterações Vocais em Idosos-RAVI)-Part II: Validity Evidence and Reliability. J Voice, v. 30, n. 2, p. 246.e19-27, 2016. DOI: 10.1016/j.jvoice.2015.04.007. Disponível em: https://PubMed.ncbi.nlm.nih.gov/25979791/. Acesso em: 24 jan. 2021.

DE ASSIS, E. N. et al. Translation and Brazilian adaptation of the Relationship Scales Questionnaire (RSQ). Trends Psychiatry Psychother., Porto Alegre, v. 41, n. 1, p. 69-77, 2019. DOI: http://dx.doi.org/10.1590/2237-6089-2018-0032. Disponível em: http://www.scielo.br/scielo.php?script=sci_arttext\&pid=S2237-60892019000100009. Acesso em: 24 jan. 2021.

DE CARVALHO, G. R. et al. Cross-cultural adaptation and reliability of the pain response to activity and position questionnaire. Adv. rheumatol., São Paulo, v. 59, 53, 2019. DOI: http://dx.doi.org/10.1186/s42358-019-0098-0. Disponível em: http://www.scielo.br/scielo.php?script=sci_arttext\&pid=S2523-31062019000100239. Acesso em: 24 jan. 2021.

DE CARVALHO, I. A. M.; MANSUR, L. L. Validation of ASHA FACS-functional assessment of communication skills for Alzheimer disease population. Alzheimer Dis. Assoc. Disord., v. 22, n. 4, p. 375-381, 2008. DOI: 10.1097/wad.0b013e31818809b2. Disponível em: https://PubMed.ncbi.nlm.nih.gov/19068501/. Acesso em: 24 jan. 2021.

DE CASTRO, S. M.; PERRACINI, M. R.; GANANCA, F. F. Versão brasileira do Dynamic Gait Index. Rev. Bras. Otorrinolaringol., São Paulo, v. 72, n. 6, p. 817-825, 2006. DOI: https://doi.org/10.1590/S0034-72992006000600014. Disponível em: https://www.scielo.br/scielo.php?script=sci_arttext\&pid=S0034-72992006000600014. Acesso em: 24 jan. 2021.

DE FREITAS, C. P. P. et al. Work-Related Flow Inventory: Evidence of Validity of the Brazilian Version. Paidéia (Ribeirão Preto), Ribeirão Preto, v. 29, e2901, 2019. DOI: https://doi.org/10.1590/1982-4327e2901. Disponível em: https://www.scielo.br/scielo.php?script=sci_abstract\&pid=S0103-863X2019000100601. Acesso em: 24 jan. 2021.

DE NEGREIROS, D. P. et al. Portuguese version of the Delirium Rating Scale-Revised-98: reliability and validity. Int. J. Geriatr. Psychiatry, v. 23, n. 5, p. 472-477, 2008. DOI: 10.1002/gps.1906. Disponível em: https://PubMed.ncbi.nlm.nih.gov/17922493/. Acesso em: 24 jan. 2021.

DE OLIVEIRA, J. B. et al. Alcohol screening instruments in elderly male: a population-based survey in metropolitan São Paulo, Brazil. Rev. Bras. Psiquiatr., São Paulo, v. 33, n. 4, p. 347352, 2011. DOI: https://doi.org/10.1590/S1516-44462011005000019. Disponível em: https://www.scielo.br/scielo.php?script=sci_arttext\&pid=S1516-44462011000400007. Acesso em: 24 jan. 2021. 
DE OLIVEIRA, G. M. et al. The applicability of the cognitive abilities screening instrumentshort (CASI-S) in primary care in Brazil. International Psychogeriatrics, v. 28, n. 1, p. 9399, 2016. DOI: 10.1017/S1041610215000642. Disponível em: https://www.cambridge.org/core/journals/international-

psychogeriatrics/article/abs/applicability-of-the-cognitive-abilities-screening-instrumentshortcasis-in-primary-care-in-brazil/77A2C496100ADA48B515F1AB8D56A2AD. Acesso em: 24 jan. 2021.

DE PAULA, J. J. et al. Clinical applicability and cutoff values for an unstructured neuropsychological assessment protocol for older adults with low formal education. PloS one, v. 8, n. 9, p. e73167, 2013. DOI: 10.1371/journal.pone.0073167. Disponível em: https://PubMed.ncbi.nlm.nih.gov/24066031/. Acesso em: 24 jan. 2021.

DE PAULA, J. J. et al. Psychometric properties of a brief neuropsychological protocol for use in geriatric populations. Rev. psiquiatr. clín., São Paulo, v. 37, n. 6, p. 251-255, 2010. DOI: http://dx.doi.org/10.1590/S0101-60832010000600002. Disponível em: http://www.scielo.br/scielo.php?script=sci_arttext\&pid=S0101-60832010000600002. Acesso em: 24 jan. 2021.

DE PAULA, J. J. et al. The Five Digits Test in the assessment of older adults with low formal education: construct validity and reliability in a Brazilian clinical sample. Trends Psychiatry Psychother., Porto Alegre, v. 39, n. 3, p. 173-179, 2017. DOI: http://dx.doi.org/10.1590/22376089-2016-0060.

Disponível

em: http://www.scielo.br/scielo.php?script=sci_arttext\&pid=S2237-60892017000300173. Acesso em: 24 jan. 2021.

DE PAULA, J. J. et al. The Stick Design Test on the assessment of older adults with low formal education: evidences of construct, criterion-related and ecological validity. International psychogeriatrics, v. 25, p. 12, p. 2057-2065, 2013. DOI: 10.1017/S1041610213001282. Disponível em: https://PubMed.ncbi.nlm.nih.gov/23905545/. Acesso em: 24 jan. 2021.

DE PAULA, J. J. et al. The tower of London test: Different scoring criteria for diagnosing Alzheimer's disease and mild cognitive impairment. Psychol. Rep., v. 110, n. 2, p. 477-488, 2012. DOI: 10.2466/03.10.13.PR0.110.2.477-488. Disponível em: https://PubMed.ncbi.nlm.nih.gov/22662402/. Acesso em: 24 jan. 2021.

DE PAULA, J. J. et al. Validity and reliability of a "simplified" version of the Taylor Complex Figure Test for the assessment of older adults with low formal education. Dement. neuropsychol., São Paulo, v. 10, n. 1, p. 52-57, 2016. DOI: https://doi.org/10.1590/s1980$57642016 \mathrm{dn} 10100010$. http://www.scielo.br/scielo.php?script=sci_arttext\&pid=S1980-57642016000100052. Acesso em: 24 jan. 2021.

DE SOUZA, D. M. et al. Validity, Reliability, and Diagnostic Accuracy of Ratings of Perceived Exertion to Identify Dependence in Performing Self-care Activities in Older Women. Experimental aging research, v. 44, n. 5, p. 397-410. 2018 DOI: 10.1080/0361073X.2018.1521492. Disponível em: https://www.tandfonline.com/doi/abs/10.1080/0361073X.2018.1521492?journalCode=uear20 . Acesso em: 24 jan. 2021. 
DE SOUZA ORLANDI, F. et al. Translation, Adaptation and Validation of Rapid Geriatric Assessment to the Brazilian Context. Journal of Nutrition, Health \& Aging, v. 22, n. 9, p. 1115-1121, 2018. DOI: 10.1007/s12603-018-1078-5. Disponível em: https://PubMed.ncbi.nlm.nih.gov/30379312/. Acesso em: 24 jan. 2021.

DIAS, E. N. et al. Validation of the advanced activities of daily living scale. Geriatric nursing, v. 40, n. 1, p. 7-12, 2019. DOI:10.1016/j.gerinurse.2018.05.008. Disponível em: https://PubMed.ncbi.nlm.nih.gov/29909023/. Acesso em: 24 jan. 2021.

DINIZ, J. G.; DA SILVA, A. C.; NÓBREGA, A. C. Quality of life and swallowing questionnaire for individuals with Parkinson's disease: development and validation. Int. J. Lang. Commun. Disord., v. 53, n. 4, p. 864-874, 2018. DOI: 10.1111/1460-6984.12395. Disponível em: https://PubMed.ncbi.nlm.nih.gov/29781565/. Acesso em: 24 jan. 2021.

FABBRI, R. M. A. et al. Validity and reliability of the Portuguese version of the Confusion Assessment Method (CAM) for the detection of delirium in the elderly. Arq. Neuro-Psiquiatr., São Paulo, v. 59, n. 2A, p. 175-179, 2001. DOI: https://doi.org/10.1590/S0004282X2001000200004. Disponível em: http://www.scielo.br/scielo.php?script=sci_arttext\&pid=S0004-282X2001000200004. Acesso em: 24 jan. 2021.

FABRICIO-WEHBE, S. C. C. et al. Cross-cultural adaptation and validity of the "Edmonton Frail Scale - EFS" in a Brazilian elderly sample. Rev. Latino-Am. Enfermagem, Ribeirão Preto, v. 17, n. 6, p. 1043-1049, 2009. DOI: https://doi.org/10.1590/S0104$11692009000600018 . \quad$ Disponível em: http://www.scielo.br/scielo.php?script=sci_arttext\&pid=S0104-11692009000600018. Acesso em: 24 jan. 2021.

FABRICIO-WEHBE, S. C. C. et al. Reproducibility of the Brazilian version of the Edmonton Frail Scale for elderly living in the community . Rev. Latino-Am. Enfermagem, Ribeirão Preto, v. 21, n. 6, p. 1330-1336, 2013. DOI: http://dx.doi.org/10.1590/0104-1169.2933.2371. Disponível em: http://www.scielo.br/scielo.php?script=sci_arttext\&pid=S010411692013000601330. Acesso em: 24 jan. 2021.

FERRARI, S. C. et al. Índice Manchester de incapacidade associada ao pé doloroso no idoso: tradução, adaptação cultural e validação para a língua portuguesa. Rev. Bras. Reumatol., São Paulo, v. 48, n. 6, p. 335-341, 2008. DOI: https://doi.org/10.1590/S0482-50042008000600006. Disponível em: http://www.scielo.br/scielo.php?script=sci_arttext\&pid=S048250042008000600006, Acesso em: 24 jan. 2021.

FERREIRA, H. G.; BARHAM, E. J.; FONTAINE, A. M. G. V. A Measure to Assess Elderly Brazilians' Involvement in Pleasant Activities: Initial Evidence of Internal and External Validity. Clinical Gerontologist, v. 38, n. 5, p. 375-394, 2015. DOI: https://doi.org/10.1080/07317115.2015.1067270. Disponível em: https://www.tandfonline.com/doi/full/10.1080/07317115.2015.1067270. Acesso em: 24 jan. 2021.

FERRETTI-REBUSTINI, R. E. L. et al. Validity of the Katz Index to assess activities of daily living by informants in neuropathological studies. Rev. esc. enferm. USP, São Paulo, v. 49, n. 6, p. 944-950, 2015. DOI: https://doi.org/10.1590/S0080-623420150000600010. Disponível 
em: http://www.scielo.br/scielo.php?script=sci_arttext\&pid=S0080-62342015000600944. Acesso em: 24 jan. 2021.

FLAKS, M. K. et al. The Short Cognitive Performance Test (SKT): a preliminary study of its psychometric properties in Brazil. International psychogeriatrics, v. 18, n. 1, p. 121-133, 2006. DOI: 10.1017/S1041610205002577. Disponível em: https://PubMed.ncbi.nlm.nih.gov/16255841/. Acesso em: 24 jan. 2021.

FLECK, M. P.; CHACHAMOVICH, E.; TRENTINI, C. Development and validation of the Portuguese version of the WHOQOL-OLD module. Rev. Saúde Pública, São Paulo, v. 40, n. 5, p. 785-791, 2006. DOI: http://dx.doi.org/10.1590/S0034-89102006000600007. Disponível em: http://www.scielo.br/scielo.php?script=sci_arttext\&pid=S0034-89102006000600007. Acesso em: 24 jan. 2021.

FLORINDO, A. A. et al. Methodology to evaluation the habitual physical activity in men aged 50 years or more. Rev. Saúde Pública, São Paulo, v. 38, n. 2, p. 307-314, 2004. DOI: https://doi.org/10.1590/S0034-89102004000200022. Disponível em: http://www.scielo.br/scielo.php?script=sci_arttext\&pid=S0034-89102004000200022. Acesso em: 24 jan. 2021.

FLORINDO, A. A. et al. Validation of the scale for evaluation of environment perception for physical activity practice in adults living in region of low socioeconomic level. Rev. bras. cineantropom. desempenho hum., Florianópolis, v. 14, n. 6, p. 647-659, 2012. DOI: https://doi.org/10.5007/1980-0037.2012v14n6p647. Disponível em: http://www.scielo.br/scielo.php?script=sci_arttext\&pid=S1980-00372012000600004. Acesso em: 24 jan. 2021.

FONSECA, R. P. et al. Brazilian version of the Protocole Montréal d'Evaluation de la Communication (Protocole MEC): normative and reliability data. Span J Psychol., v. 11, n. 2, p. 678-688, 2008. PMID: 18988453. Disponível em: https://PubMed.ncbi.nlm.nih.gov/18988453/. Acesso em: 24 jan. 2021.

FRANCO, C. R. C. et al. Reliability and validity of a scale for measurement of trunk mobility in Parkinson's disease: Trunk Mobility Scale. Arq. Neuro-Psiquiatr., São Paulo, v. 69, n. 4, p. 636-641, 2011. DOI: https://doi.org/10.1590/S0004-282X2011000500012. Disponível em: http://www.scielo.br/scielo.php?script=sci_arttext\&pid=S0004-282X2011000500012. Acesso em: 24 jan. 2021.

FREIRE, A. N. et al. Validity and Reliability of the Short Physical Performance Battery in Two Diverse Older Adult Populations in Quebec and Brazil. Journal of Aging \& Health, v. 24, n. 5, p. 863-878, 2012. DOI: 10.1177/0898264312438551. Disponível em: https://PubMed.ncbi.nlm.nih.gov/22422762/. Acesso em: 24 jan. 2021.

GAMBARO, R. C. et al. Questionnaire of pain in geriatrics: adaptation proposal for portuguese language of Geriatric Pain Measure (GPM). Rev. Bras. Med., v. 66, n. 3, p. 62-65, 2009. Disponível em: http://bases.bireme.br/cgibin/wxislind.exe/iah/online/?IsisScript=iah/iah.xis\&src=google\&base=LILACS\&lang=p\&nex tAction=lnk\&exprSearch=512194\&indexSearch=ID. Acesso em: 24 jan. 2021. 
GARCIA, I. F. F. et al. A study of measurement properties of the Life-Space Assessment questionnaire in older adults with chronic obstructive pulmonary disease. Clin. Rehabil., v. 32, n. 10, p. 1374-1382, 2018. DOI: 10.1177/0269215518780488. Disponível em: https://PubMed.ncbi.nlm.nih.gov/29865890/. Acesso em: 24 jan. 2021.

GEERINCK, A. et al. Standard error of measurement and smallest detectable change of the Sarcopenia Quality of Life (SarQoL) questionnaire: An analysis of subjects from 9 validation studies. PLoS ONE, v. 14, n. 4, 2019. DOI: 10.1371/journal.pone.0216065. Disponível em: https://PubMed.ncbi.nlm.nih.gov/31034498/. Acesso em: 24 jan. 2021.

GIAVONI, A. et al. Elaboração e validação da Escala de Depressão para Idosos. Cad. Saúde Pública, Rio de Janeiro, v. 24, n. 5, p. 975-982, 2008. DOI: https://doi.org/10.1590/S0102311X2008000500004. Disponível

em: http://www.scielo.br/scielo.php?script=sci_arttext\&pid=S0102-311X2008000500004. Acesso em: 25 jan. 2021.

GONCALVES, D. M.; CLONINGER, C. R. Validation and normative studies of the Brazilian Portuguese and American versions of the Temperament and Character Inventory - Revised (TCI-R). Journal of affective disorders, v. 124, n. 1-2, p. 126-33, 2010. DOI:10.1016/j.jad.2009.11.007. Disponível em: https://PubMed.ncbi.nlm.nih.gov/?term=Cloninger+CR\&cauthor_id=19942293. Acesso em: 25 jan. 2021.

GORENSTEIN, C. et al. Validation of the Portuguese version of the Social Adjustment Scale on Brazilian samples. Journal of affective disorders, v. 69, n. 1-3, p. 167-75, 2002. DOI:10.1016/s0165-0327(01)00300-7. Disponível em: https://PubMed.ncbi.nlm.nih.gov/12103463/. Acesso em: 25 jan. 2021.

GUEDES, M. B. O. G. et al. Validation of a questionnaire for the evaluation of informal social support for the elderly: section 1. Rev. bras. geriatr. gerontol., Rio de Janeiro, v. 21, n. 6, p. 647-656, 2018. DOI: https://doi.org/10.1590/1981-22562018021.180119. Disponível em: http://www.scielo.br/scielo.php?script=sci_arttext\&pid=S1809-98232018000600647. Acesso em: 25 jan. 2021.

HUA, F. Y. et al. Tradução e adaptação do examen géronto-psychomoteur para o português do Brasil. Fisioterapia Brasil, v. 20, n. 2, p. 213-221, 2019. DOI: http://dx.doi.org/10.33233/fb.v20i2.2787. Disponível em: http://www.portalatlanticaeditora.com.br/index.php/fisioterapiabrasil/article/view/2787\#: :tex $\mathrm{t}=\mathrm{O} \% 20$ objetivo $\% 20 \mathrm{do} \% 20$ presente $\% 20$ estudo,avalia\%C3\%A7\%C3\%A3o\%20funcional $\% 2$ 0do\%20idoso\%20brasileiro. Acesso em: 25 jan. 2021.

JACINTO, A. F. et al. Dementia Rating Scale psychometric study and its applicability in long term care institutions in Brazil. Einstein (Sao Paulo), v. 10, n. 3, p. 318-322, 2012. DOI: https://doi.org/10.1590/S1679-45082012000300011. Disponível em: https://www.scielo.br/scielo.php?pid=S1679-45082012000300011\&script=sci_abstract. Acesso em: 25 jan. 2021.

JOÃO, T. M. S. et al. Validity of the Brazilian version of the Godin-Shephard Leisure-Time Physical Activity Questionnaire. Cad. saúde pública, Rio de Janeiro, v. 31, n. 9, p. 1825-1838, 2015. DOI: https://doi.org/10.1590/0102-311X00189713 Disponível em: 
https://www.scielo.br/scielo.php?script=sci_arttext\&pid=S0102-311X2015000901825. Acesso em: 25 jan. 2021.

JOMAR, R. T.; LOURENCO, R. A.; LOPES, C. S. Acurácia da versão brasileira do Functional Activities Questionnaire no rastreio de demência. Rev. Enf. Ref., Coimbra, v. serIV, n. 21, p. 25-33, 2019. DOI: http://dx.doi.org/10.12707/RIV19022. Disponível em http://www.scielo.mec.pt/scielo.php?script=sci_arttext\&pid=S0874-02832019000200003. Acesso em: 25 jan. 2021.

JOMAR, R. T.; LOURENCO, R. A.; LOPES, C. S. Estrutura dimensional da versão brasileira do Functional Activities Questionnaire (FAQ-BR). Cad. Saúde Pública, Rio de Janeiro, v. 34, n. 11, e00209917, 2018. DOI: http://dx.doi.org/10.1590/0102-311x00209917. Disponível em: http://www.scielo.br/scielo.php?script=sci_arttext\&pid=S0102-311X2018001104001. Acesso em: 25 jan. 2021.

KANO, M. Y.; SANTOS, M. A.; PILLON, S. C. Uso do álcool em idosos: validação transcultural do Michigan Alcoholism Screening Test - Geriatric Version (MAST-G). Rev. esc. enferm. USP, São Paulo, v. 48, n. 4, p. 649-656, 2014. DOI: https://doi.org/10.1590/S0080623420140000400011.

http://www.scielo.br/scielo.php?script=sci_arttext\&pid=S0080-62342014000400649. Acesso em: 25 jan. 2021.

KRIEGER, D. M. et al. Parkinson's Disease Impulsive-Compulsive Disorders Questionnaire Current Short (QUIP-CS) - Translation and validation of content of Portuguese Version. J. bras. psiquiatr., Rio de Janeiro, v. 66, n. 2, p. 111-115, 2017 . DOI: https://doi.org/10.1590/0047-2085000000158. Disponível em: http://www.scielo.br/scielo.php?script=sci_arttext\&pid=S0047-20852017000200111. Acesso em: 25 jan. 2021.

KUMMER, A. et al. Evaluation of fatigue in Parkinson's disease using the Brazilian version of Parkinson's Fatigue Scale. Acta neurologica Scandinavica, v. 123, n. 2, p. 130-136, 2011. DOI: 10.1111/j.1600-0404.2010.01364.x. Disponível em: https://PubMed.ncbi.nlm.nih.gov/20456242/. Acesso em: 25 jan. 2021.

KUMMER, A.; CARDOSO, F.; TEIXEIRA, A. L. Frequency of social phobia and psychometric properties of the Liebowitz social anxiety scale in Parkinson's disease. Movement disorders, v. 23, n. 12, p. 1739-1743, 2008. DOI: 10.1002/mds.22221. Disponível em: https://PubMed.ncbi.nlm.nih.gov/18661550/. Acesso em: 25 jan. 2021.

KUMMER, A.; CARDOSO, F.; TEIXEIRA, A. L. Generalized anxiety disorder and the Hamilton Anxiety Rating Scale in Parkinson's disease. Arq. Neuro-Psiquiatr., São Paulo, v. 68, n. 4, p. 495-501, 2010. DOI: http://dx.doi.org/10.1590/S0004-282X2010000400005. Disponível em: http://www.scielo.br/scielo.php?script=sci_arttext\&pid=S0004282X2010000400005. Acesso em: 25 jan. 2021.

KUZNIER, T. P. Tradução, adaptação e validação da UCLA Loneliness Scale(version 3) para o português do Brasil em uma amostra de idosos. 2016. Tese (Doutorado em Enfermagem) - Universidade Federal de Minas Gerais, 2016. DOI: http://hdl.handle.net/1843/ANDO-AEHLB3. Disponível em: https://repositorio.ufmg.br/handle/1843/ANDO-AEHLB3. Acesso em: 25 jan. 2021. 
LAGUARDIA, J. et al. Psychometric evaluation of the SF-36 (v.2) questionnaire in a probability sample of Brazilian households: results of the survey Pesquisa Dimensões Sociais das Desigualdades (PDSD), Brazil, 2008. Health Qual Life Outcomes, v. 9, n. 61, p. 1-10, 2011. DOI: 10.1186/1477-7525-9-61. Disponível em: https://www.arca.fiocruz.br/handle/icict/3859. Acesso em: 25 jan. 2021.

LAUS, M. F. et al. Brazilian version of the Multidimensional Body-Self Relations Questionnaire-Appearance Scales (MBSRQ-AS): translation and psychometric properties in adults. Eating and weight disorders, v. 25, n. 5, p. 1253-1266, 2020. DOI: 10.1007/s40519019-00758-w. Disponível em: https://PubMed.ncbi.nlm.nih.gov/31364031/. Acesso em: 25 jan. 2021.

LEITE, K. S. B. et al. Boston Naming Test (BNT) Original, Brazilian Adapted Version and Short Forms: Normative Data for Illiterate and Low-Educated Older Adults. International Psychogeriatrics, v. 29, n. 5, p. 825-833, 2017. DOI: 10.1017/S1041610216001952. Disponibilidade em: https://www.cambridge.org/core/journals/internationalpsychogeriatrics/article/abs/boston-naming-test-bnt-original-brazilian-adapted-version-andshort-forms-normative-data-for-illiterate-and-loweducated-olderadults/FD80C3A117EE4F8976C3600493D3C41F. Acesso em: 25 jan. 2021.

LIMA, F. M. et al. Quality of life amongst older Brazilians: a cross-cultural validation of the CASP-19 into Brazilian-Portuguese. PloS one, v. 9, n. 4, e94289, 2014, DOI:10.1371/journal.pone.0094289. Disponível https://PubMed.ncbi.nlm.nih.gov/24740240/. Acesso em: 25 jan. 2021.

LIMA, J. E. M.; PALACIOS, S.; WENDER, M. C. O. Quality of life in menopausal women: a Brazilian Portuguese version of the Cervantes Scale. Scientific World Journal, v. 2012, 620519, 2012. DOI: 10.1100/2012/620519 Disponível em: https://www.ncbi.nlm.nih.gov/pmc/articles/PMC3317546/. Acesso em: 26 jan. 2021.

LINO, V. T. S. et al. Adaptação transcultural da Escala de Independência em Atividades da Vida Diária (Escala de Katz). Cad. Saúde Pública, Rio de Janeiro, v. 24, n. 1, p. 103-112, 2008. DOI: https://doi.org/10.1590/S0102-311X2008000100010. Disponível em: http://www.scielo.br/scielo.php?script=sci_arttext\&pid=S0102-311X2008000100010. Acesso em: 26 jan. 2021.

LISBOA, L. L. et al. Tradução, adaptação e validação da versão brasileira do questionário Utian Quality of Life para avaliação da qualidade de vida no climatério. Rev. Bras. Ginecol. Obstet., Rio de Janeiro, v. 37, n. 11, p. 520-525, 2015. DOI: https://doi.org/10.1590/SO100$720320150005438 . \quad$ Disponível em: http://www.scielo.br/scielo.php?script=sci_arttext\&pid=S0100-72032015001100520. Acesso em: 27 jan. 2021.

LOPES, R. A. et al. Psychometric properties of the Brazilian version of the Pain Catastrophizing Scale for acute low back pain. Arq. Neuro-Psiquiatr., São Paulo, v. 73, n. 5, p. 436-445, 2015. DOI: https://doi.org/10.1590/0004-282X20150026. Disponível em: http://www.scielo.br/scielo.php?script=sci_arttext\&pid=S0004-282X2015000500012. Acesso em: 27 jan. 2021. 
LOPES, A. R.; TRELHA, C. S. Translation, cultural adaptation and evaluation of the psychometric properties of the Falls Risk Awareness Questionnaire (FRAQ): FRAQ-Brazil. Braz. J. Phys. Ther., São Carlos, v. 17, n. 6, p. 593-605, 2013. DOI: https://doi.org/10.1590/S1413-35552012005000128. Disponível em: http://www.scielo.br/scielo.php?script=sci_arttex\&pid=S1413-35552013000600593. Acesso em: 27 jan. 2021.

LOURENCO, R. A.; VERAS, R. P. Mini-Mental State Examination: psychometric characteristics in elderly outpatients. Rev. Saúde Pública, São Paulo, v. 40, n. 4, p. 712-719, 2006. DOI: https://doi.org/10.1590/S0034-89102006000500023. Disponível em: http://www.scielo.br/scielo.php?script=sci_arttext\&pid=S0034-89102006000500023. Acesso em: 27 jan. 2021.

LOURENCO, R. A.; VERAS, R. P. Mini-Mental State Examination: psychometric characteristics in elderly outpatients. Rev. Saúde Pública, São Paulo, v. 40, n. 4, p. 712-719, 2006. DOI: https://doi.org/10.1590/S0034-89102006000500023. Disponível em: http://www.scielo.br/scielo.php?script=sci_arttext\&pid=S0034-89102006000500023. Acesso em: 27 jan. 2021.

LUFT, C. D. B. et al. Versão brasileira da Escala de Estresse Percebido: tradução e validação para idosos. Rev. Saúde Pública, São Paulo, v. 41, n. 4, p. 606-615, 2007. DOI: https://doi.org/10.1590/S0034-89102007000400015. Disponível em: http://www.scielo.br/scielo.php?script=sci_arttext\&pid=S0034-89102007000400015. Acesso em: 27 jan. 2021.

MACEDO MONTANO, M. B. M.; RAMOS, L. R. Validade da versão em português da Clinical Dementia Rating. Rev. Saúde Pública, São Paulo, v. 39, n. 6, p. 912-917, 2005. DOI: https://doi.org/10.1590/S0034-89102005000600007. Disponível em: http://www.scielo.br/scielo.php?script=sci_arttext\&pid=S0034-89102005000600007. Acesso em: 27 jan. 2021.

MACHADO, M. C.; LOPES, G. H.; MARCHINI, L. Oral health of Alzheimer's patients in São José dos Campos, Brazil. Geriatr. Gerontol. Int., v. 12, n. 2, p. 265-270, 2012. DOI: 10.1111/j.1447-0594.2011.00763.x. Disponível

em: https://PubMed.ncbi.nlm.nih.gov/22032653/. Acesso em: 27 jan. 2021.

MAIA, A. L. G. et al. Aplicação da versão brasileira da escala de avaliação clínica da demência (Clinical Dementia Rating - CDR) em amostras de pacientes com demência. Arq. NeuroPsiquiatr., São Paulo, v. 64, n. 2b, p. 485-489, 2006. DOI: https://doi.org/10.1590/S0004282X2006000300025. Disponível em: http://www.scielo.br/scielo.php?script=sci_arttext\&pid=S0004-282X2006000300025. Acesso em: 27 jan. 2021.

MAIA, A. C. et al. Cross-cultural adaptation and analysis of the psychometric properties of the Balance Evaluation Systems Test and MiniBESTest in the elderly and individuals with Parkinson's disease: application of the Rasch model. Braz. J. Phys. Ther., São Carlos, v. 17, n. 3, p. 195-217, 2013. DOI: https://doi.org/10.1590/S1413-35552012005000085. Disponível em: http://www.scielo.br/scielo.php?script=sci_arttext\&pid=S1413-35552013000300195. Acesso em: 27 jan. 2021. 
MARANHAO NETO, G. A.; LUZ, L. G. O.; FARINATTI, P. T. V. Diagnostic accuracy of pre-exercise screening questionnaire: Emphasis on educational level and cognitive status. Arch. Gerontol. Geriatr., v. 57, n. 2, p. 211-214, 2013. DOI: https://doi.org/10.1016/j.archger.2013.03.008. Disponível em: https://www.sciencedirect.com/science/article/abs/pii/S0167494313000411. Acesso em: 27 jan. 2021.

MARGIS, R. et al. Psychometric properties of the Parkinson's Disease Sleep Scale--Brazilian version. Parkinsonism \& related disorders, v. 15, n. 7, p. 495-9, 2009. DOI: 10.1016/j.parkreldis.2008.12.008. Disponível em: https://PubMed.ncbi.nlm.nih.gov/19211294/. Acesso em: 27 jan. 2021.

MARTINEZ, M. C. et al. Transcultural adaptation of the Johns Hopkins Fall Risk Assessment Tool. Rev. Latino-Am. Enfermagem, Ribeirão Preto, v. 24, e2783, 2016. DOI: https://doi.org/10.1590/1518-8345.1158.2783. Disponível em: http://www.scielo.br/scielo.php?script=sci_arttext\&pid=S0104-11692016000100404. Acesso em: 27 jan. 2021.

MARTINEZ, M. C. et al. Validade e confiabilidade da versão brasileira da Johns Hopkins Fall Risk Assessment Tool para avaliação do risco de quedas. Rev. bras. epidemiol., São Paulo, v. 22, e190037, 2019. DOI: https://doi.org/10.1590/1980-549720190037. Disponível em: http://www.scielo.br/scielo.php?script=sci_arttext\&pid=S1415-790X2019000100436. Acesso em: 27 jan. 2021.

MARTINEZ-MARTIN, P. et al. International study on the psychometric attributes of the nonmotor symptoms scale in Parkinson disease. Neurology, v. 73, n. 19, p. 1584-91, 2009. DOI:10.1212/WNL.0b013e3181c0d416. Disponível em: https://PubMed.ncbi.nlm.nih.gov/19901251/\#: :text=The\%20SEM\%20was\%2013.91\%20for, nonmotor\%20symptoms\%20in\%20Parkinson\%20disease. Acesso em: 27 jan. 2021.

MASSENA, P. N. et al. Validation of the Brazilian Portuguese Version of Geriatric Anxiety Inventory--GAI-BR. International Psychogeriatrics, v. 27, n. 7, p. 1113-1119, 2015. DOI: 10.1017/S1041610214001021. Disponível em: https://PubMed.ncbi.nlm.nih.gov/24946782/. Acesso em: 27 jan. 2021.

MELO, R. L. P. et al. Psychometric properties of the complete version of the World Health Organization Quality of Life Assessment (WHOQOL-OLD): reduced response scale. Psicol. Reflex. Crit., Porto Alegre, v. 31, n. 4, 2018. DOI: https://doi.org/10.1186/s41155-018-00841. Disponível em: http://www.scielo.br/scielo.php?script=sci_arttext\&pid=S010279722018000103102. Acesso em: 27 jan. 2021.

MEMÓRIA, C. M. et al. Brief screening for mild cognitive impairment: validation of the Brazilian version of the Montreal cognitive assessment. Int. J. Geriatr. Psychiatry, v. 28, n. 1, p. 34-40, 2013 2013. DOI: 10.1002/gps.3787. Disponível em: https://PubMed.ncbi.nlm.nih.gov/22368034/. Acesso em: 27 jan. 2021.

MEURER, S.T. et al. Validade de construto e consistência interna da escala de autoestima de Rosenberg para uma população de idosos brasileiros praticantes de atividades físicas. Motri., Vila Real, v. 8, n. 4, p. 5-15, 2012. DOI: http://dx.doi.org/10.6063/motricidade.8(4).1548. 
Disponível em: http://www.scielo.mec.pt/scielo.php?script=sci_arttext\&pid=S1646107X2012000400002. Acesso em: 27 jan. 2021.

MIGUEL, F. K. et al. A Brazilian Investigation of the 36- and 16-Item Difficulties in Emotion Regulation Scales. J. Clin. Psychol., v. 73, n. 9, p. 1146-1159, 2017. DOI: 10.1002/jclp.22404. Disponível em: https://PubMed.ncbi.nlm.nih.gov/27717003/. Acesso em: 27 jan. 2021.

MINOSSO, J. S. M. et al. Validation of the Barthel Index in elderly patients attended in outpatient clinics, in Brazil. Acta paul. enferm., São Paulo, v. 23, n. 2, p. 218-223, 2010. DOI: https://doi.org/10.1590/S0103-21002010000200011. Disponível em http://www.scielo.br/scielo.php?script=sci_arttext\&pid=S0103-21002010000200011. Acesso em: 27 jan. 2021.

MIRANDA, D. D. C.; BRUCKI, S. M. D.; YASSUDA, M. S. The Mini-Addenbrooke's Cognitive Examination (M-ACE) as a brief cognitive screening instrument in Mild Cognitive Impairment and mild Alzheimer's disease. Dement. Neuropsychol., São Paulo, v. 12, n. 4, p. 368-373, 2018. DOI: http://dx.doi.org/10.1590/1980-57642018dn12-040005. Disponível em: https://www.scielo.br/scielo.php?pid=S1980-57642018000400368. Acesso em: 27 jan. 2021.

MOGRABI, D. C. et al. Cross-cultural adaptation and validation of the Brazilian version of the Beliefs about Emotions Scale. Trends psychiatry psychother., v. 40, n. 1, p. 21-28, 2018. DOI: http://dx.doi.org/10.1590/2237-6089-2017-0064. Disponível em: https://www.scielo.br/scielo.php?pid=S2237-60892018000100021 Acesso em: 27 jan. 2021.

MOLINA, M. D. C. B. et al. Reprodutibilidade e validade relativa do Questionário de Frequência Alimentar do ELSA-Brasil. Cad. saúde pública, v. 29, n. 2, p. 379-389, 2013. DOI: https://doi.org/10.1590/S0102-311X2013000200024. Disponível em: https://www.scielo.br/scielo.php?script=sci_arttext\&pid=S0102-311X2013000200024. Acesso em: 27 jan. 2021.

MONTEIRO, D. R.; ALMEIDA, M. A.; KRUSE, M. H. L. Tradução e adaptação transcultural do instrumento Edmonton Symptom Assessment System para uso em Cuidados Paliativos. Rev Gaucha Enferm., v. 34, n. 2, p. 163-171, 2013. DOI: https://doi.org/10.1590/S198314472013000200021. Disponível em: https://www.scielo.br/scielo.php?pid=S198314472013000200021. Acesso em: 27 jan. 2021.

MOTTA, T. S.; GAMBARO, R. C.; SANTOS, F. C. Pain measurement in the elderly: evaluation of psychometric properties of the Geriatric Pain Measure - Portuguese version. Rev. dor, São Paulo, v. 16, n. 2, p. 136-141, 2015. DOI: http://dx.doi.org/10.5935/18060013.20150026. Disponível em: http://www.scielo.br/scielo.php?script=sci_arttext\&pid=S1806-00132015000200136. Acesso em: 27 jan. 2021.

NERI, A. L. et al. New semantic-cultural validation and psychometric study of the CASP-19 scale in adult and elderly Brazilians. Cadernos de saude publica, v. 34, n. 10, e00181417, 2018. DOI: https://doi.org/10.1590/0102-311x00181417. Disponível em: https://www.scielo.br/scielo.php?pid=S0102-311X2018001004002. Acesso em: 27 jan. 2021.

NEUBERN, P. C. B. Funcionalidade e função executiva em idosos saudáveis e portadores de demência na doença de Alzheimer: estudo de validação do Executive Function 
Performance Test-Br. 2018. Tese (Doutorado em Ciências) - Faculdade de Medicina da USP, 2018. DOI: 10.11606/T.5.2018.tde-02072018-114315. Disponível em: https://www.teses.usp.br/teses/disponiveis/5/5142/tde-02072018-114315/pt-br.php. Acesso em: 27 jan. 2021.

NEVES, M. B. et al. Cross-cultural adaptation of the Western Aphasia Battery - Revised screening test to Brazilian Portuguese: a preliminary study. CoDAS, São Paulo, v. 26, n. 1, p. 38-45, 2014. DOI: https://doi.org/10.1590/s2317-17822014000100006. Disponível em: https://www.scielo.br/scielo.php?script=sci_arttext\&pid=S2317-17822014000100038. Acesso em: 27 jan. 2021.

NOVELLI, M. M. P. C. et al. Cross-cultural adaptation of the quality of life assessment scale on Alzheimer disease. Arq. Neuro-Psiquiatr., v. 63, n. 2, p. 201-206, 2005. DOI: https://doi.org/10.1590/S0004-282X2005000200002. Disponível em: https://www.scielo.br/scielo.php?script=sci_arttext\&pid=S0004-282X2005000200002. Acesso em: 27 jan. 2021.

NOVELLI, M. M. P. C., NITRINI, R.; CARAMELLI, P. Validation of the Brazilian version of the quality of life scale for patients with Alzheimer's disease and their caregivers (QOL-AD). Aging \& Mental Health, v. 14, n. 5, p. 624-631, 2010. DOI: 10.1080/13607861003588840. Disponível em: https://PubMed.ncbi.nlm.nih.gov/20480421/. Acesso em: 27 jan. 2021.

NUNES, C. H. S. S.; HUTZ, C. S. Construção e validação da escala fatorial de Socialização no modelo dos Cinco Grandes Fatores de Personalidade. Psicol. reflex. Crit., Porto Alegre, v. 20, n. 1, p. 20-25, 2007. DOI: https://doi.org/10.1590/S0102-79722007000100004. Disponível em: https://www.scielo.br/scielo.php?script=sci_arttext\&pid=S0102-79722007000100004. Acesso em: 27 jan. 2021.

NUNES, S. A. N. et al. Psychometric properties of the Brazilian version of the lived experience component of the Spiritual Health And Life-Orientation Measure (SHALOM). Psicol. Reflex. Crit., Porto Alegre, v. 31, n. 2, 2018. DOI: https://doi.org/10.1186/s41155-018-0083-2. Disponível em: https://www.scielo.br/scielo.php?script=sci_arttext\&pid=S010279722018000103101. Acesso em: 27 jan. 2021.

OLISAN, G. O. S. et al. Psychometric evidence of the Brazilian version of Driving Cognitions Questionnaire (DCQ). Comprehensive Psychiatry, v. 60, p. 156-160, 2015. DOI: https://doi.org/10.1016/j.comppsych.2014.08.044. Disponível em: https://www.sciencedirect.com/science/article/abs/pii/S0010440X14002363. Acesso em: 27 jan. 2021.

ORLANDI, F. S. et al. The evaluation of the level of hope of elderly chronic kidney disease patients undergoing hemodialysis. Rev. Esc. Enferm. USP, v. 46, n. 4, p. 900-905, 2012. DOI: 10.1590/s0080-62342012000400017. Disponível https://PubMed.ncbi.nlm.nih.gov/23018400/. Acesso em: 27 jan. 2021.

OSORIO, F. L. et al. Psychometrics properties of early trauma inventory self report - short form (ETISR-SR) for the Brazilian context. PloS ONE, v. 8, n. 10, e76337, 2013. DOI: 10.1371/journal.pone.0076337. Disponível em: https://PubMed.ncbi.nlm.nih.gov/24098478/. Acesso em: 27 jan. 2021. 
OSTI, R. F. I.; GARCIA, L. M. T.; FLORINDO, A. A. Validation of the 24-hour physical activity recall in elderly adults. Rev. bras. cineantropom. desempenho hum., Florianópolis, v. 16, n. 1, p. 15-26, 2014. DOI: https://doi.org/10.5007/1980-0037.2014v16n1p15. Disponível em: http://www.scielo.br/scielo.php?script=sci_arttext\&pid=S1980-00372014000100015. Acesso em: 27 jan. 2021.

PASCHOAL, S. M. P.; JACOB FILHO, W.; LITVOC, J. Development of Elderly Quality of Life Index - EqoLI: item reduction and distribution into dimensions. Clinics, São Paulo, v. 63, n. 2, p. 179-188, 2008. DOI: https://doi.org/10.1590/S1807-59322008000200005. Disponível em: http://www.scielo.br/scielo.php?script=sci_arttext\&pid=S1807-59322008000200005. Acesso em: 27 jan. 2021.

PAES, T. et al. Londrina activities of daily living protocol: Reproducibility, validity, and reference values in physically independent adults age 50 years and older. Respir. Care, v. 62, n. 3, p. 298-306, 2017. DOI: 10.4187/respcare.05059. Disponível em: https://PubMed.ncbi.nlm.nih.gov/28143963/\#: :text=Conclusions\%3A\%20The\%20Londrina $\%$ 20ADL\%20protocol,protocol\%27s\%20results\%20in\%20clinical\%20practice. Acesso em: 27 jan. 2021.

PAIXAO JR, C. M. et al. Adaptação transcultural para o Brasil do instrumento Caregiver Abuse Screen (CASE) para detecção de violência de cuidadores contra idosos. Cad. Saúde Pública, Rio de Janeiro, v. 23, n. 9, p. 2013-2022, 2007. DOI: http://dx.doi.org/10.1590/S0102311X2007000900010. Disponível em: http://www.scielo.br/scielo.php?script=sci_arttext\&pid=S0102-311X2007000900010. Acesso em: 27 jan. 2021.

PARADELA, E. M. P.; LOPES, C. S.; LOURENCO, R. A. Reliability of the Brazilian version of the Cambridge Cognitive Examination Revised CAMCOG-R. Arq. Neuro-Psiquiatr., São Paulo, v. 67, n. 2b, p. 439-444, 2009. DOI: http://dx.doi.org/10.1590/S0004282X2009000300013. Disponível em: http://www.scielo.br/scielo.php?script=sci_arttext\&pid=S0004-282X2009000300013. Acesso em: 27 jan. 2021.

PARADELA, E. M. P.; LOURENCO, R. A.; VERAS, R. P. Validation of geriatric depression scale in a general outpatient clinic. Rev. Saúde Pública, São Paulo, v. 39, n. 6, p. 918-923, 2005. DOI: https://doi.org/10.1590/S0034-89102005000600008. Disponível em: http://www.scielo.br/scielo.php?script=sci_arttext\&pid=S0034-89102005000600008. Acesso em: 27 jan. 2021.

PAULA, J. J. et al. Development, validity, and reliability of the General Activities of Daily Living Scale: a multidimensional measure of activities of daily living for older people. Rev. Bras. Psiquiatr., São Paulo, v. 36, n. 2, p. 143-152, 2014. DOI: https://doi.org/10.1590/15164446-2012-1003. http://www.scielo.br/scielo.php?script=sci_arttext\&pid=S1516-44462014000200143. Acesso em: 27 jan. 2021.

PAULA, J. J. et al. Evaluating Language Comprehension in Alzheimer's disease: the use of the Token Test. Arq. Neuro-Psiquiatr., São Paulo, v. 70, n. 6, p. 435-440, 2012. DOI: https://doi.org/10.1590/S0004-282X2012000600010. 
http://www.scielo.br/scielo.php?script=sci_arttext\&pid=S0004-282X2012000600010. Acesso em: 27 jan. 2021.

PEDREIRA, R. B. S. et al. Content validity of the Geriatric Health Assessment Instrument. Einstein (Sao Paulo), v. 14, n. 2, p. 158-177, 2016. DOI: https://doi.org/10.1590/S167945082016AO3455.

Disponível

em: https://www.scielo.br/scielo.php?script=sci_arttext\&pid=S1679-45082016000200009. Acesso em: 27 jan. 2021.

PEREIRA, L. S. M. et al. Adaptação transcultural e análise da confiabilidade do Southampton Assessment of Mobility para avaliar a mobilidade de idosos brasileiros com demência. Cad. Saúde Pública, Rio de Janeiro, v. 22, n. 10, p. 2085-2095, 2006. DOI: https://doi.org/10.1590/S0102-311X2006001000014. Disponível em: http://www.scielo.br/scielo.php?script=sci_arttext\&pid=S0102-311X2006001000014. Acesso em: 27 jan. 2021.

PEREIRA, F. S. et al. Cross-cultural adaptation, reliability and validity of the DAFS-R in a sample of Brazilian older adults. Arch. Clin. Neuropsychol., v. 25, n. 4, p. 335-343, 2010. DOI: 10.1093/arclin/acq029. Disponível em: https://PubMed.ncbi.nlm.nih.gov/20484096/. Acesso em: 28 jan. 2021.

PEREIRA, L. S. M. et al. Factorial analysis of the Multidimensional Health Locus of control scale - form C for elderly. Brazilian Journal of Physical Therapy, São Carlos, v. 15, n. 5, p. 363-370, 2011. DOI: https://doi.org/10.1590/S1413-35552011005000013. Disponível em: https://www.scielo.br/scielo.php?script=sci_arttext\&pid=S1413-35552011000500005. Acesso em: 28 jan. 2021.

PEREIRA, D. A. et al. Philadelphia Brief Assessment of Cognition in healthy and clinical Brazilian sample. Arq Neuropsiquiatr., São Paulo, v. 70, n. 3, p. 175-9, 2012. DOI: https://doi.org/10.1590/S0004-282X2012005000001. Disponível em: https://www.scielo.br/scielo.php?script=sci_arttext\&pid=S0004-282X2012000300004. Acesso em: 28 jan. 2021.

PERNAMBUCO, L. A. et al. Screening for Voice Screening for Voice Disorders in Older Adults (Rastreamento de Alterações Vocais em Idosos-RAVI)-Part II: Validity Evidence and Reliability. J. Voice, v. 30, n. 2, p. 246.e9-17, 2016. DOI: 10.1016/j.jvoice.2015.04.007. Disponível em: https://PubMed.ncbi.nlm.nih.gov/25979791/. Acesso em: 28 jan. 2021.

PERRACINI, M. R. et al. Diagnostic Accuracy of the Short Physical Performance Battery for Detecting Frailty in Older People. Phys. Ther., v. 100, n. 1, p. 90-98, 2020. DOI: 10.1093/ptj/pzz154. Disponível em: https://PubMed.ncbi.nlm.nih.gov/31612228/. Acesso em: 28 jan. 2021.

PERROCO, T. R. et al. Performance of Brazilian long and short IQCODE on the screening of dementia in elderly people with low education. International Psychogeriatrics, v. 21, n. 3, p. 531-538, 2009. DOI: 10.1017/S1041610209008849. Disponível em: https://PubMed.ncbi.nlm.nih.gov/19323868/\#: :text=Conclusions\%3A\%20The\%20long\%2C $\% 20$ short\%20and,with\%20low\%20levels\%20of\%20education. Acesso em: 28 jan. 2021.

PINHO, M. X. et al. Confiabilidade e validade da escala de depressão geriátrica em idosos com doença arterial coronariana. Arq. bras. Cardiol., São Paulo, v. 94, n. 5, p. 570-579, 2010. DOI: 
http://dx.doi.org/10.1590/S0066-782X2010005000032.

Disponível

em: https://www.scielo.br/scielo.php?pid=S0066-782X2010000500001. Acesso em: 28 jan. 2021.

PINTO, T. C. C. et al. Accuracy and Psychometric Properties of the Brazilian Version of the Montreal Cognitive Assessment as a Brief Screening Tool for Mild Cognitive Impairment and Alzheimer's Disease in the Initial Stages in the Elderly. Dement. Geriatr. Cogn. Disord., v. 47, n. 4, p. 366-374, 2019. DOI: 10.1159/000501308. Disponível em: https://PubMed.ncbi.nlm.nih.gov/31466064/\#: :text=Conclusions\%3A\%20The\%20results\%2 0of $\% 20$ the,to $\% 20$ discriminate $\% 20$ between $\% 20$ cognitively $\% 20$ healthy. Acesso em: 28 jan. 2021.

PORTO, C. S. et al. Brazilian version of the Mattis dementia rating scale diagnosis of mild dementia in Alzheimer's disease. Arquivos de neuro-psiquiatria, v. 61, n. 2, p. 339-345, 2003. DOI: https://doi.org/10.1590/S0004-282X2003000300004. Disponível em: https://www.scielo.br/scielo.php?script=sci_arttext\&pid=S0004-282X2003000300004. Acesso em: 28 jan. 2021.

PORTUGAL, M. G. et al. Validation of Montgomery-Åsberg Rating Scale and Cornell Scale for Depression in Dementia in Brazilian elderly patients. Int. Psychogeriatr., v. 24, n. 8, p. 1291-1298, 2012. DOI: 10.1017/S1041610211002250. Disponível em: https://PubMed.ncbi.nlm.nih.gov/22185723/. Acesso em: 28 jan. 2021.

PRINCE, M. et al. Effects of education and culture on the validity of the Geriatric Mental State and its AGECAT algorithm. Br. J. Psychiatry, v. 185, p. 429-436, 2004. DOI: 10.1192/bjp.185.5.429. Disponível em: https://PubMed.ncbi.nlm.nih.gov/15516553/. Acesso em: 28 jan. 2021.

REICHENHEIM, M. E.; PAIXAO JR., C. M.; MORAES, C. L. Portuguese (Brazil) crosscultural adaptation of the Hwalek-Sengstock Elder Abuse Screening Test (H-S/EAST) used to identify risk of violence against the elderly. Cad. Saúde Pública, Rio de Janeiro, v. 24, n. 8, p. 1801-1813, 2008. DOI: https://doi.org/10.1590/S0102-311X2008000800009. Disponível em: https://www.scielo.br/scielo.php?pid=S0102-311X2008000800009. Acesso em: 28 jan. 2021.

REICHENHEIM, M. E.; PAIXAO JR., C. M.; MORAES, C. L. Reassessing the construct validity of a Brazilian version of the instrument Caregiver Abuse Screen (CASE) used to identify risk of domestic violence against the elderly. J. Epidemiol. Community Health, v. 63, n. 11, p. 878-883, 2009. DOI: 10.1136/jech.2008.084095. Disponível em: https://PubMed.ncbi.nlm.nih.gov/19622518/. Acesso em: 28 jan. 2021.

REIS, M. S.; REIS, R. S.; HALLAL, P. C. Validity and reliability of a physical activity social support assessment scale. Rev. Saude Publica, v. 45, n. 2, p. 294-301, 2011. DOI: 10.1590/s0034-89102011000200008. Disponível em: https://PubMed.ncbi.nlm.nih.gov/21412569/. Acesso em: 28 jan. 2021.

RIBEIRO, C. C.; NERI, A. L.; YASSUDA, M. S. Semantic-cultural validation and internal consistency analysis of the Purpose in Life Scale for brazilian older adults. Dement. neuropsychol., São Paulo, v. 12, n. 3, p. 244-249, 2018. DOI: https://doi.org/10.1590/198057642018dn12-030004. Disponível em: http://www.scielo.br/scielo.php?script=sci_arttext\&pid=S1980-57642018000300244. Acesso em: 28 jan. 2021. 
RIBEIRO FILHO, S. T.; LOURENÇO, R. A. O desempenho do Mini-Cog em uma amostra de idosos com baixo nível educacional. Dement. Neuropsychol., São Paulo, v. 3, n. 2, p. 81-87, 2009. DOI: http://dx.doi.org/10.1590/S1980-57642009DN30200003. Disponível em: https://www.scielo.br/scielo.php?pid=S1980-

57642009000200081\&script=sci_abstract\&tlng=pt. Acesso em: 28 jan. 2021.

RICCI, N. A. et al. Evaluation of properties of the Vestibular Disorders Activities of Daily Living Scale (Brazilian version) in an elderly population. Brazilian Journal of Physical Therapy, São Carlos, v. 18, n. 2, p. 174-182, 2014. DOI: http://dx.doi.org/10.1590/S141335552012005000144. https://www.scielo.br/scielo.php?script=sci_arttext\&pid=S1413-35552014000200174. Acesso em: 28 jan. 2021.

RODRIGUES, G. R. et al. Cross-cultural adaptation and validation of the episodic autobiographic memory interview for Brazilian Portuguese. Arq. Neuropsiquiatr., São Paulo, v. 73, n. 8, p. 676-680, 2015. DOI: http://dx.doi.org/10.1590/0004-282X20150084. Disponível em: https://www.scielo.br/scielo.php?script=sci_arttext\&pid=S0004-282X2015000800676. Acesso em: 28 jan. 2021.

SACCOMANN, I. C. R. S.; CINTRA, F. A.; GALLANI, M. C. B. J. Health-related quality of life among the elderly with heart failure: a generic measurement. Sao Paulo Med. J., São Paulo, v. 128, n. 4, p. 192-196, 2010. DOI: http://dx.doi.org/10.1590/S1516-31802010000400003. Disponível em: http://www.scielo.br/scielo.php?script=sci_arttext\&pid=S151631802010000400003. Acesso em: 28 jan. 2021.

SACCOMANN, I. C. R. S.; CINTRA, F. A.; GALLANI, M. C. B. J. Psychometric properties of the Minnesota Living with Heart Failure--Brazilian version--in the elderly. Qual. Life Res., v. 16, n. 6, p. 997-1005, 2007. DOI: 10.1007/s11136-007-9170-z. Disponível em: https://PubMed.ncbi.nlm.nih.gov/17534737/. Acesso em: 28 jan. 2021.

SAENGER, A. L. F.; CALDAS, C. P.; RAÎCHE, M.; DA MOTTA, L. B. Identifying the loss of functional independence of older people residing in the community: Validation of the PRISMA-7 instrument in Brazil. Archives of Gerontology \& Geriatrics, v. 74, p. 62-67, 2018. DOI: 10.1016/j.archger.2017.09.008. Disponível em: https://PubMed.ncbi.nlm.nih.gov/29031102/. Acesso em: 28 jan. 2021.

SALES, M. C. V. et al. A useful and brief cognitive assessment for advanced dementia in a population with low levels of education. Dement. Geriatr. Cogn. Disord., v. 32, n. 5, p. 295300, 2011. DOI: 10.1159/000335358. Disponível em: https://PubMed.ncbi.nlm.nih.gov/22262084/. Acesso em: 28 jan. 2021.

SANCHEZ, M. A. S.; LOURENÇO, R. A. Screening for dementia: Brazilian version of the Informant Questionnaire on Cognitive Decline on the Elderly and its psychometric properties. Geriatrics \& Gerontology International, v. 13, n. 3, p. 687-693, 2013. DOI: 10.1111/j.14470594.2012.00966.x. Disponível em: https://PubMed.ncbi.nlm.nih.gov/23186020/. Acesso em: 28 jan. 2021.

SANTIAGO, L. M. et al. Predictive validity of the Brazilian version of the Tilburg Frailty Indicator for adverse health outcomes in older adults. Arch. Gerontol. Geriatr., v. 76, p. 114- 
119, 2018. DOI: 10.1016/j.archger.2018.02.013. Disponível em: https://PubMed.ncbi.nlm.nih.gov/29494871/. Acesso em: 28 jan. 2021.

SANTIAGO, L. M. et al. Psychometric properties of the Brazilian version of the Tilburg frailty indicator (TFI). Archives of Gerontology \& Geriatrics, v. 57, n. 1, p. 39-45, 2013. DOI: 10.1016/j.archger.2013.03.001. Disponível em: https://PubMed.ncbi.nlm.nih.gov/23538005/. Acesso em: 28 jan. 2021.

SANTOS, M. P. et al. Comparison between two functional mobility scales for Parkinson's disease directly applied to physical therapy practice: cross-cultural adaptation and measurement properties. Eur. J. Phys. Rehabil. Med., v. 53, n. 5, p. 664-675, 2017. DOI: 10.23736/S19739087.16.04148-4. Disponível em: https://PubMed.ncbi.nlm.nih.gov/27050084/. Acesso em: 28 jan. 2021.

SANTOS, R. L. et al. MacArthur Competence Assessment Tool for Treatment in Alzheimer disease: cross-cultural adaptation. Arquivos de neuro-psiquiatria, São Paulo, v. 75, n. 1, p. 36-43, 2017. DOI: https://doi.org/10.1590/0004-282x20160181. Disponível em: https://www.scielo.br/scielo.php?script=sci_arttext\&pid=S0004-282X2017000100036. Acesso em: 28 jan. 2021.

SANTOS, M. P. et al. Parkinson Activity Scale: cross-cultural adaptation and reliability of the Brazilian version. Geriatrics \& gerontology international, v. 15, n. 1, p. 89-95, 2015. DOI: 10.1111/ggi.12235. Disponível em: https://PubMed.ncbi.nlm.nih.gov/24456087/. Acesso em: 28 jan. 2021.

SANTOS, M. T. F.; SOUGEY, E. B.; ALCHIERI, J. C. Validity and reliability of the screening test for Alzheimer's disease with proverbs (STADP) for the elderly. Arquivos de neuropsiquiatria, São Paulo, v. 67, n. 3, p. 836-842, 2009. DOI: https://doi.org/10.1590/S0004282X2009000500011. Disponível em: https://www.scielo.br/scielo.php?script=sci_arttext\&pid=S0004-282X2009000500011. Acesso em: 28 jan. 2021.

SARAIVA, M. D. et al. AMPI-AB validity and reliability: a multidimensional tool in resourcelimited primary care settings. BMC Geriatr., v. 20, n. 1, p. 124, 2020. DOI: 10.1186/s12877020-01508-9. Disponível em: https://PubMed.ncbi.nlm.nih.gov/32228469/. Acesso em: 28 jan. 2021.

SARDA JÚNIOR, J. et al. Psychometric properties of the DASS-Depression scale among a Brazilian population with chronic pain. Journal of Psychosomatic Research, v. 64, n. 1, p. 25-31, 2008. DOI: 10.1016/j.jpsychores.2007.05.015. Disponível em: https://PubMed.ncbi.nlm.nih.gov/18157996/. Acesso em: 28 jan. 2021.

SARDÁ JÚNIOR, J. J. et al. Validação do questionário de incapacidade Roland Morris para dor em geral. Rev. dor, v. 11, n. 1, 2010. Disponível em: http://bases.bireme.br/cgibin/wxislind.exe/iah/online/?IsisScript=iah/iah.xis\&src=google\&base=LILACS\&lang=p\&nex tAction=lnk\&exprSearch=562427\&indexSearch=ID. Acesso em: 28 jan. 2021.

SAURIN, G.; CROSSETTI, M. G O. Reliability and validity of the Pain Assessment Tool in Confused Older Adults - IADIC. Rev. Gaúcha Enferm., Porto Alegre, v. 34, n. 4, p. 68-74, 2013. DOI: https://doi.org/10.1590/S1983-14472013000400009. Disponível em: 
https://www.scielo.br/scielo.php?pid=S1983-14472013000400009\&script=sci_abstract. Acesso em: 28 jan. 2021.

SCALZO, P. L. et al. Validation of the Brazilian version of the Berg balance scale for patients with Parkinson's disease. Arq. Neuropsiquiatr., São Paulo, v. 67, n. 3, p. 831-835, 2009. DOI: http://dx.doi.org/10.1590/S0004-282X2009000500010. Disponível em: https://www.scielo.br/scielo.php?script=sci_arttext\&pid=S0004-282X2009000500010. Acesso em: 28 jan. 2021.

SCAZUFCA, M. et al. Limitations of the Mini-Mental State Examination for screening dementia in a community with low socioeconomic status: results from the Sao Paulo Ageing \& Health Study. Eur. Arch. Psychiatry Clin. Neurosci., v. 259, n. 1, p. 8-15, 2009. DOI: 10.1007/s00406-008-0827-6. Disponível em: https://PubMed.ncbi.nlm.nih.gov/18560791/. Acesso em: 28 jan. 2021.

SCAZUFCA, M. et al. Validity of the self reporting questionnaire-20 in epidemiological studies with older adults: results from the Sao Paulo Ageing \& Health Study. Soc. Psychiatry Psychiatr. Epidemiol., v. 44, n. 3, p. 247-54, 2009. DOI: 10.1007/s00127-008-0425-y. Disponível em: https://PubMed.ncbi.nlm.nih.gov/18777144/. Acesso em: 28 jan. 2021.

SEWO SAMPAIO, P. Y. et al. Validation and translation of the Kihon Checklist (frailty index) into Brazilian Portuguese. Geriatr. Gerontol. Int., v. 14, n. 3, p. 561-569, 2014. DOI: 10.1111/ggi.12134. Disponível em: https://PubMed.ncbi.nlm.nih.gov/23992357/. Acesso em: 28 jan. 2021.

SILBERMAN, C. D. et al. Recognizing depression in patients with Parkinson's disease: accuracy and specificity of two depression rating scale. Arq. Neuro-Psiquiatr., São Paulo , v. 64, n. 2b, p. 407-411, 2006. DOI: http://dx.doi.org/10.1590/S0004-282X2006000300011. Disponível em: http://www.scielo.br/scielo.php?script=sci_arttext\&pid=S0004282X2006000300011. Acesso em: 28 jan. 2021.

SILVA, P. A. B. et al. Cut-off point for WHOQOL-bref as a measure of quality of life of older adults. Rev. Saúde Pública, São Paulo, v. 48, n. 3, p. 390-397, 2014. DOI: https://doi.org/10.1590/S0034-8910.2014048004912. Disponível em: http://www.scielo.br/scielo.php?script=sci_arttext\&pid=S0034-89102014000300390. Acesso em: 28 jan. 2021.

SILVA, S. M. et al. VES-13 and WHOQOL-bref cutoff points to detect quality of life in older adults in primary health care. Rev. Saúde Pública, São Paulo, v. 53, p. 26, 2019. DOI: https://doi.org/10.11606/s1518-8787.2019053000802. Disponível em: http://www.scielo.br/scielo.php?script=sci_arttext\&pid=S0034-89102019000100222. Acesso em: 28 jan. 2021.

SILVA FILHO, C. R. et al. Climacteric symptoms and quality of life: validity of women's health questionnaire. Rev. Saúde Pública, v. 39, n. 3, p. 333-9, 2005. DOI: https://doi.org/10.1590/S0034-89102005000300002 Disponível em: https://www.scielo.br/scielo.php?script=sci_arttext\&pid=S0034-89102005000300002. Acesso em: 28 jan. 2021. 
SILVEIRA, M. B. et al. Construction and validation of content of one instrument to assess falls in the elderly. Einstein (Sao Paulo), São Paulo, v. 16, n. 2, p. eAO4154, 2018. DOI: https://doi.org/10.1590/s1679-45082018ao4154. Disponível em: https://www.scielo.br/scielo.php?script=sci_arttext\&pid=S1679-45082018000200207. Acesso em: 28 jan. 2021.

SIMÕES, M. S. M. et al. Life-Space Assessment questionnaire: Novel measurement properties for Brazilian community-dwelling older adults. Geriatrics \& gerontology international, v. 18, n. 5, p. 783-789, 2018. DOI: 10.1111/ggi.13263. Disponível em: https://PubMed.ncbi.nlm.nih.gov/29372585/. Acesso em: 28 jan. 2021.

SIMON, S. S. et al. Metamemory and aging: Psychometric properties of the Brazilian version of the Multifactorial Memory Questionnaire for elderly. Dementia \& neuropsychologia, São Paulo, v. 10, n. 2, p. 113-126, 2016. DOI: https://doi.org/10.1590/S1980-57642016DN1002007. Disponível em: https://www.scielo.br/scielo.php?pid=S198057642016000200113. Acesso em: 28 jan. 2021.

SIQUEIRA, T. H.; VILA, V. S. C.; WEISS, M. E. Cross-cultural adaptation of the instrument Readiness for Hospital Discharge Scale - Adult Form. Rev. Bras. Enferm., Brasília, v. 71, n. 3, p. 983-991, 2018. DOI: http://dx.doi.org/10.1590/0034-7167-2017-0241. Disponível em: http://www.scielo.br/scielo.php?script=sci_arttext\&pid=S0034-71672018000300983. Acesso em: 28 jan. 2021.

SOBREIRA, E. et al. Screening of cognitive impairment in patients with Parkinson's disease: diagnostic validity of the Brazilian versions of the Montreal Cognitive Assessment and the Addenbrooke's Cognitive Examination-Revised. Arq Neuropsiquiatr., São Paulo, v. 73, n. 11, p. 929-933, 2015. DOI: https://doi.org/10.1590/0004-282X20150156. Disponível em: https://www.scielo.br/scielo.php?script=sci_arttext\&pid=S0004-282X2015001100929.

Acesso em: 28 jan. 2021.

SOUZA, A. C.; MAGALHAES, L. C.; TEIXEIRA-SALMELA, L. F. Adaptação transcultural e análise das propriedades psicométricas da versão brasileira do Perfil de Atividade Humana. Cad. Saúde Pública, Rio de Janeiro, v. 22, n. 12, p. 2623-2636, 2006. DOI: https://doi.org/10.1590/S0102-311X2006001200012. Disponível em: http://www.scielo.br/scielo.php?script=sci_arttext\&pid=S0102-311X2006001200012. Acesso em: 28 jan. 2021.

SOUSA, R. M. M. et al. Feasibility and reliability of the elderly version of the Camberwell Assessment of Needs (CANE): results from the São Paulo Ageing \& Health Study. Rev. Bras. Psiquiatr., São Paulo, v. 31, n. 1, p. 34-38, 2009. DOI: https://doi.org/10.1590/S1516$44462009000100009 . \quad$ Disponível em: http://www.scielo.br/scielo.php?script=sci_arttext\&pid=S1516-44462009000100009. Acesso em: 28 jan. 2021.

SPAGNUOLO, D. L. et al. Walking for the assessment of balance in healthy subjects older than 40 years. Gerontology, v. 56, n. 5, p. 467-473, 2010. DOI: 10.1159/000275686. Disponível em: https://PubMed.ncbi.nlm.nih.gov/20090294/. Acesso em: 28 jan. 2021.

STELLA, F. et al. The Brazilian version of the Neuropsychiatric Inventory-Clinician rating scale (NPI-C): reliability and validity in dementia. International Psychogeriatrics, v. 25, n. 9, 
p. 1503-1511, 2013. DOI: 10.1017/S1041610213000811. Disponível em: https://PubMed.ncbi.nlm.nih.gov/23763895/. Acesso em: 28 jan. 2021.

STELLA, F. et al. Validation of the Brazilian version of the apathy inventory. Int. J. Geriatr. Psychiatry, v. 28, n. 9, p. 979-986, 2013. DOI: http://dx.doi.org/10.1002/gps.3917. Disponível em: https://repositorio.unesp.br/handle/11449/76371?show=full. Acesso em: 28 jan. 2021.

SWAROWSKY, A. et al. Cross cultural adaptations and psychometric domains of Brazilian version of PROFILE PD for Parkinson's disease. Disability and rehabilitation, v. 39, n. 17, p. 1759-1770, 2017. DOI: 10.1080/09638288.2016.1209695. Disponível em: https://PubMed.ncbi.nlm.nih.gov/27685369/. Acesso em: 28 jan. 2021.

TAVARES, D. R. B.; SANTOS, F. C. Locomotive syndrome in the elderly: translation, cultural adaptation, and Brazilian validation of the tool 25-Question Geriatric Locomotive Function Scale. Rev. bras. Reumatol., São Paulo, v. 57, n. 1, p. 56-63, 2017. DOI: https://doi.org/10.1016/j.rbre.2016.07.015. Disponível em: https://www.scielo.br/scielo.php?script=sci_arttext\&pid=S0482-50042017000100056. Acesso em: 28 jan. 2021.

TAVARES, L. S. et al. Reliability, validity, interpretability and responsiveness of the DEMMI mobility index for Brazilian older hospitalized patients. PloS ONE, v. 15, n. 3, p. e0230047, 2020. DOI: 10.1371/journal.pone.0230047. Disponível em: https://PubMed.ncbi.nlm.nih.gov/32187212/. Acesso em: 28 jan. 2021.

TEIXEIRA, L. F. et al. Cross-cultural adaptation, validity and reproducibility of the Back Beliefs Questionnaire among older Brazilians with acute low back pain. A cross-sectional study. Sao Paulo Medical Journal, São Paulo, v. 138, n. 4, p. 287-296, 2020. DOI: https://doi.org/10.1590/1516-3180.2019.0542.r2.16042020. Disponível em: https://www.scielo.br/scielo.php?script=sci_abstract\&pid=S1516-31802020000400287.

Acesso em: 28 jan. 2021.

TEIXEIRA-SALMELA, L. F. et al. Adaptação do Perfil de Saúde de Nottingham: um instrumento simples de avaliação da qualidade de vida. Cad. Saúde Pública, Rio de Janeiro, v. 20, n. 4, p. 905-914, 2004. DOI: https://doi.org/10.1590/S0102-311X2004000400004. Disponível em: https://www.scielo.br/scielo.php?script=sci_arttext\&pid=S0102311X2004000400004. Acesso em: 28 jan. 2021.

THE, K. B. et al. Pain assessment in elderly with dementia: Brazilian validation of the PACSLAC scale. Einstein (São Paulo), São Paulo, v. 14, n. 2, p. 152-157, 2016. DOI: https://doi.org/10.1590/S1679-45082016AO3628. Disponível em: http://www.scielo.br/scielo.php?script=sci_arttext\&pid=S1679-45082016000200008. Acesso em: 28 jan. 2021.

TOLEDO, F. O. et al. Cross-cultural adaptation and validation of the brazilian version of the wisconsin brief pain questionnaire. Journal of Pain \& Symptom Management, v. 46, n. 1, p. 121-130, 2013. DOI: 10.1016/j.jpainsymman.2012.07.017. Disponível em: https://PubMed.ncbi.nlm.nih.gov/23219149/. Acesso em: 28 jan. 2021.

VALADARES, A. L. R. et al. Adaptação sociocultural do short personal experiences questionnaire (SPEQ) no Brasil. Rev. Bras. Ginecol. Obstet., Rio de Janeiro, v. 32, n. 2, p. 72 - 
76, 2010. DOI: https://doi.org/10.1590/S0100-72032010000200004. Disponível em: https://www.scielo.br/scielo.php?script=sci_arttext\&pid=S0100-72032010000200004. Acesso em: 29 jan. 2021.

VALDERRAMAS, S.; FERES, A. C.; MELO, A. Reliability and validity study of a BrazilianPortuguese version of the fatigue severity scale in Parkinson's disease patients. Arq. NeuroPsiquiatr., São Paulo, v. 70, n. 7, p. 497-500, 2012. DOI: https://doi.org/10.1590/S0004282X2012000700005.

https://www.scielo.br/scielo.php?script=sci_arttext\&pid=S0004-282X2012000700005.

Acesso em: 29 jan. 2021.

VALERA, G. G. et al. Cultural adaptation of the scale Pain Assessment in Advanced Dementia - PAINAD to Brazil. Revista da Escola de Enfermagem da USP, São Paulo, v. 48, n. 3, p. 462-468, 2014. DOI: https://doi.org/10.1590/S0080-623420140000300011. Disponível em: https://www.scielo.br/scielo.php?script=sci_arttext\&pid=S0080-62342014000300462. Acesso em: 29 jan. 2021.

PARADELA, E. M. P.; LOURENCO, R. A.; VERAS, R. P. Validation of geriatric depression scale in a general outpatient clinic. Rev. Saúde Pública, São Paulo, v. 39, n. 6, p. 918-923, 2005. DOI: https://doi.org/10.1590/S0034-89102005000600008. Disponível em: http://www.scielo.br/scielo.php?script=sci_arttext\&pid=S0034-89102005000600008. Acesso em: 29 jan. 2021.

VAZQUEZ, A. C. S. et al. Adaptation and Validation of the Brazilian Version of the Utrecht Work Engagement Scale. Psico USF, Itatiba, v. 20, n. 2, p. 207-217, 2015. DOI: https://doi.org/10.1590/1413-82712015200202. Disponível em: https://www.scielo.br/scielo.php?script=sci_arttext\&pid=S1413-82712015000200207. Acesso em: 29 jan. 2021.

VENTURA, M. M.; BOTTINO, C. M. C. Reliability study of the Brazilian version of a structured interview for the diagnosis of dementia. Rev. Assoc. Med. Bras., São Paulo, v. 47, n. 2, p. 110-116, 2001. DOI: https://doi.org/10.1590/S0104-42302001000200028. Disponível em: https://www.scielo.br/scielo.php?pid=S0104-42302001000200028. Acesso em: 29 jan. 2021.

VICTOR, J. F.; XIMENES, L. B.; ALMEIDA, P. C. Adaptação transcultural para o Brasil da Exercise Benefits/Barriers Scale (EBBS) para aplicação em idosos: uma avaliação semântica. Cad. Saúde Pública, Rio de Janeiro, v. 24, n. 12, p. 2852-2860, 2008. DOI: https://doi.org/10.1590/S0102-311X2008001200014. Disponível em: http://www.scielo.br/scielo.php?script=sci_arttext\&pid=S0102-311X2008001200014. Acesso em: 29 jan. 2021.

VIGNOLA, R. C. B.; TUCCI, A. M. Adaptation and validation of the depression, anxiety and stress scale (DASS) to Brazilian Portuguese. J Affect Disord., v. 155, p. 104-109, 2014. DOI: http://dx.doi.org/10.1016/j.jad.2013.10.031. Disponível http://repositorio.unifesp.br/handle/11600/37373. Acesso em: 29 jan. 2021.

VIRTUOSO JUNIOR, J. S.; GUERRA, R. O. Reliability of functional fitness tests in women from 60 to 80 years. Motricidade, v. 7, n. 2, p. 7-13, 2011. DOI: 
https://doi.org/10.6063/motricidade.106.

Disponível

em: https://revistas.rcaap.pt/motricidade/article/view/106. Acesso em: 29 jan. 2021.

VIRUÉS-ORTEGA, J. et al. Cross-cultural evaluation of the modified Parkinson Psychosis Rating Scale across disease stages. Mov Disord., v. 25, n. 10, p. 1391-1398, 2010. DOI: 10.1002/mds.23081. Disponível em: https://PubMed.ncbi.nlm.nih.gov/20310036/. Acesso em: 29 jan. 2021.

VIVEIRO, L. A. P. et al. Reliability, Validity, and Ability to Identity Fall Status of the Berg Balance Scale, Balance Evaluation Systems Test (BESTest), Mini-BESTest, and BriefBESTest in Older Adults Who Live in Nursing Homes. J. Geriatr. Phys. Ther., v. 42, n. 4, p. E45-E54, 2019. DOI: 10.1519/JPT.0000000000000215. Disponível em: https://PubMed.ncbi.nlm.nih.gov/30407272/. Acesso em: 29 jan. 2021.

YASSUDA, M. S. et al. Psychometric characteristics of the Rivermead Behavioural Memory Test (RBMT) as an early detection instrument for dementia and mild cognitive impairment in Brazil. International Psychogeriatrics, v. 22, n. 6, p. 1003-1011, 2010. DOI: 10.1017/S1041610210001055. Disponível em: https://PubMed.ncbi.nlm.nih.gov/20598195/. Acesso em: 29 jan. 2021.

YOKOMIZO, J. E. et al. Cognitive screening test in primary care: cut points for low education. Rev. Saúde Pública, São Paulo, v 52, 88, 2018. DOI: https://doi.org/10.11606/s15188787.2018052000462. https://www.scielo.br/scielo.php?script=sci_arttext\&pid=S0034-89102018000100276. Acesso em: 29 jan. 2021.

ZIBETTI, M. R. et al. Evidence of clinical, criterion, and convergent validity of the Brazilian version of the picture Free and Cued Selective Reminding Test with Immediate Recall (pFCSRT-IR). Psychology \& Neuroscience, v. 12, n. 2, p. 169-179, 2019. DOI: https://doi.org/10.1037/pne0000157. Disponível em: https://psycnet.apa.org/record/201913182-001. Acesso em: 29 jan. 2021.

ZUCOLOTO, M L. et al. Construct validity of the Brazilian version of the Medical Outcomes Study Social Support Survey (MOS-SSS) in a sample of elderly users of the primary healthcare system. Trends in psychiatry and psychotherapy, Porto Alegre, v. 41, n. 4, p. 340-347, 2019. DOI: https://doi.org/10.1590/2237-6089-2018-0092. Disponível em: https://www.scielo.br/scielo.php?pid=S2237-60892019005010101. Acesso em: 29 jan. 2021.

\section{EVIDÊNCIAS DE VALIDADE DOS INSTRUMENTOS DE AVALIAÇÃO DE} ANSIEDADE, DEPRESSÃO E ESTRESSE PARA IDOSOS BRASILEIROS: UMA
REVISÃO SISTEMÁTICA

\section{RESUMO}

Este estudo objetivou, realizar uma revisão sistemática sobre as evidências de validade e as propriedades psicométricas dos instrumentos de avaliação de ansiedade, depressão e estresse 
para idosos brasileiros. Foram analisados os estudos de evidências de validade originais (desenvolvimento) e cross-culturais (tradução e adaptação) dos instrumentos recuperados. Esta revisão foi conduzida baseada nas recomendações Cochrane e PRISMA. A partir das buscas na base PubMed foram recuperados 178 estudos. Foram somados a estes, 21 estudos buscados sistematicamente no primeiro artigo deste projeto e ainda, nove estudos selecionados por busca manual em artigos de interesse. Foram identificados um total de 208 estudos, sendo que 52 foram excluídos por duplicidade. Seguiram para análise de títulos e resumos, 156 artigos. Destes, foram excluídos 111, restando 45 para leitura na íntegra e análise de elegibilidade. Após a leitura completa dos artigos, foram excluídos oito estudos, por não contemplarem idosos ou idosos brasileiros em sua amostra, restando 37 estudos para a síntese analítica. Dos 37 estudos recuperados, foram identificados 25 instrumentos. Os dados analisados foram dispostos em três etapas, sendo a primeira demonstrando as evidências recuperadas, as características das amostras e as características dos instrumentos, a segunda, por análise metodológica de cada estudo e por fim, a terceira, com a análise das etapas percorridas em cada estudo, para reunir as evidências de validade dos instrumentos em questão. O uso de instrumentos tem impacto direto e importante para a população idosa, aqui em destaque, mas não somente. Desprezar a literatura contemporânea e continuar utilizando desenhos de pesquisa baseado em modelos ultrapassados é irresponsável. Não se trata de descartar o que já foi alcançado, mas sim, questionar e aprimorar o conhecimento disponível.

\begin{abstract}
This study aimed to carry out a systematic review of the evidence of validity and the psychometric properties of the instruments for assessing anxiety, depression and stress for elderly Brazilians. Original (development) and cross-cultural (translation and adaptation) validity evidence studies of the recovered instruments were analyzed. This review was conducted based on the Cochrane and PRISMA recommendations. From the searches in the PubMed database, 178 studies were retrieved. In addition, 21 studies were systematically searched for in the first article of this project and nine studies selected by manual search for articles of interest. A total of 208 studies were identified, of which 52 were excluded due to duplication. 156 articles followed for the analysis of titles and abstracts. Of these, 111 were excluded, with 45 remaining for full reading and eligibility analysis. After reading the articles in full, eight studies were excluded, as they did not include elderly or Brazilian elderly in their sample, leaving 37 studies for the analytical synthesis. Of the 37 studies retrieved, 25
\end{abstract}


instruments were identified. The analyzed data were arranged in three stages, the first showing the recovered evidence, the characteristics of the samples and the characteristics of instruments, the second, by methodological analysis of each study and finally, the third, with the analysis of the steps taken in each study, to gather evidence of validity of the instruments in question. The use of instruments has a direct and important impact for the elderly population, highlighted here, but not only. To despise contemporary literature and to continue using research designs based on outdated models is irresponsible. It is not a question of discarding what has already been achieved, but of questioning and improving the available knowledge.

\subsection{INTRODUÇÃO}

Ansiedade, depressão e estresse são alvos de diferentes estudos com idosos, devido ao impacto que estes transtornos podem ocasionar, como limitações nas atividades de vida diária. A atenção aos sintomas destes transtornos é de suma importância, para um cuidado adequado com a saúde mental do idoso. Estudos relacionando a dança como fator protetivo (OLIVEIRA et al., 2017); investigando o estilo de coping para lidar com as questões do envelhecimento (SOUZA-TALARICO et al., 2009); analisando a prevalência destes transtornos em idosos da comunidade (MAXIMIANO-BARRETO; FERMOSELI, 2017); correlacionando estes transtornos com a saúde bucal (MENEZES-SILVA et al., 2016); ou mesmo associando os níveis destes transtornos à pandemia do COVID-19 (CORRÊA et al., 2020), são fontes constantes de orientação aos profissionais e estudiosos da temática, que baseiam suas intervenções e estimam seus resultados, a partir de avaliações por meio de instrumentos medida/psicométricos. Estes estudos são cada vez mais comuns na literatura, pois buscam incessantemente compreender as correlações destas variáveis, bem como seu impacto na população idosa e formas preventivas e interventivas de lidar com estes transtornos. Estas variáveis, foram ainda, fonte de interesse de pelo menos 11 instrumentos em 21 estudos capturados no primeiro artigo desta dissertação, o que demonstra a variedade de ferramentas para a compreensão da manifestação da variável em si e por mais oportuno que pareça a variabilidade, pode gerar confusão na hora da escolha para utilização com a população de interesse, o que traz a necessidade do pesquisador/profissional/aplicador compreender aspectos básicos dos instrumentos do medida, como sua estrutura, aplicação, tempo de preenchimento, correção e classificações, mas principalmente, que saiba identificar, se este instrumento é um instrumento válido para a sua população de interesse. 
O conceito de validade discutido na literatura passou por uma evolução temporal que pode ser rememorada nas edições dos Standards for educational and psychological testing, desenvolvidos pela AERA, APA e NCME (1954; 1965; 1985; 1999; 2014). O conceito perpassou por diferentes propostas, compreendida por fim, como um sistema unitário, ou seja, acredita-se que as evidências de validade podem ser estudadas/analisadas a partir de cinco grandes fontes, evidências de conteúdo, evidências de processo de resposta, evidências de estrutura interna, evidências de relações com outras variáveis e evidências de consequências da testagem. É importante destacar que, tais evidências se referem aos escores dos testes e sua interpretação e não ao teste em si (ANDRADE; VALENTINI, 2018). Apoiando o conceito de que validade pertence então, às inferências ou interpretações a partir das pontuações e não aos próprios testes (MESSICK, 1989). Pode-se falar em uma análise de baixo ou alto índice de evidências de validade, ou seja, como um processo. O que conflita com conceitos anteriores, denominados "tipos de validade", mas que continuam sendo referenciados por grande parte dos estudos psicométricos.

Tomadas de decisões são apoiadas a partir de instrumentos de medida, logo, se preocupar com as evidências de validade dos instrumentos envolvidos neste processo de avaliação é essencial (NORONHA; VENDRAMINI, 2003; TANAKA; TAMAKI, 2012). Os dados gerados pelos instrumentos, bem como os estudos psicométricos, são importantíssimos, visto as recomendações que são ofertadas a partir deste tipo de estudo. Portanto, avançar com análises pormenorizadas das evidências de validade destes instrumentos especificamente (ansiedade, depressão e estresse), atende uma necessidade global e crescente da garantia de medidas de qualidade para esta população e avança em consonância com a proposta de um olhar macro-micro das etapas percorridas, que designaram essas evidências, à luz da ciência psicométrica.

\subsection{OBJETIVOS}

\subsubsection{Geral}

Realizar uma revisão sistemática sobre as evidências de validade e as propriedades psicométricas dos instrumentos de avaliação de ansiedade, depressão e estresse para idosos brasileiros. 


\subsubsection{Específicos}

Investigar os tipos de evidências de validade dos instrumentos de ansiedade, depressão e estresse em idosos brasileiros; e identificar os procedimentos e técnicas utilizadas nos processos de validação.

\subsection{MÉTODO}

Por meio de uma revisão sistemática foram analisados os estudos de adaptação, desenvolvimento e validação dos instrumentos das variáveis latentes: ansiedade, depressão e estresse para idosos brasileiros. Esta revisão foi conduzida baseada nas recomendações Cochrane Handbook for Systematic Reviews of Interventions (HIGGINS et al., 2019) e Preferred Reporting Items for Systematic Reviews and Meta-Analyses: The PRISMA Statement (MOHER et al., 2009).

\subsubsection{Pergunta de pesquisa}

A questão que norteou o processo desta revisão sistemática foi: "Quais são as evidências psicométricas disponíveis, dos instrumentos de avaliação de ansiedade, depressão e estresse para idosos brasileiros?”. A partir desta pergunta foi construída a estratégia PICO, aonde: P (população/paciente) - idosos brasileiros; I (intervenção/exposição) - instrumento de avaliação de ansiedade e/ou depressão e/ou estresse; C (grupo comparador) - não aplicável; O (desfecho) - estudo de evidência de validade: tradução e adaptação transcultural e desenvolvimento.

\subsubsection{Estratégia de busca}

Inicialmente foram considerados os 17 instrumentos abaixo, identificados em 21 estudos psicométricos incluídos no primeiro artigo deste projeto:

- Center for Epidemiologic Studies Depression Scale (CES-D);

- Depression Anxiety Stress Scale (DASS);

- Depression Anxiety Stress Scale (DASS-Depressão);

- Depression Scale for the Elderly (DSE);

- Geriatric Anxiety Inventory (GAI-BR);

- Geriatric Depression Scale (GDS-1);

- Geriatric Depression Scale (GDS-4);

- Geriatric Depression Scale (GDS-10); 
- Geriatric Depression Scale (GDS-15);

- Geriatric Depression Scale (GDS-30);

- Geriatric Emotional Assessment of Pain (GEAP-b);

- Hamilton Anxiety Rating Scale (HAM-A);

- Liebowitz Social Anxiety Scale (LSAS);

- Montgomery-Asberg Depression Rating Scale (MADRS);

- Patient Health Questionnaire (PHQ-2);

- Patient Health Questionnaire (PHQ-9);

- Perceived Stress Scale (PSS).

Para uma análise mais ampla, foram considerados outros oito instrumentos que não eram alvo dos estudos psicométricos recuperados, mas compuseram o método destes mesmos estudos e se propunham a avaliar as variáveis em questão, logo, foram adicionados para esta análise:

- Adult Stressors Inventory (ASI);

- Baptista Depression Scale - Elderly Version (EBADEP ID);

- Cornell Scale for Depression in Dementia (CSDD);

- Generalized Anxiety Disorder (GAD);

- Hamilton Depression Scale (HAM-D);

- Hospital Anxiety and Depression Scale (HADS);

- State-Trait Anxiety Inventory (STAI);

- Zung Self-Rating Depression Scale (SDS).

Portanto, as buscas foram realizadas dia 05 de janeiro de 2021, unicamente na base de dados PubMed, seguindo uma estratégia adaptada para cada instrumento. Foi realizada também, uma busca manual para recuperação de qualquer evidência que pudesse integrar esta análise. O algoritmo base foi estruturado da seguinte forma: (((("NOME DO INSTRUMENTO"[Title/Abstract])) AND (psychometr* OR valid*)) AND (aged OR elderly OR "older people")) AND (brazil*). A primeira parte trata do nome do instrumento, o seguimento do algoritmo indica o foco e limites da busca. Assim, apenas a primeira parte da estratégia sofreu alteração nas buscas. Como exemplo: (((("Adult Stressors Inventory" OR “ASI”[Title/Abstract])) AND (psychometr* OR valid*)) AND (aged OR elderly OR "older 
people")) AND (brazil*). O procedimento foi realizado para todos os instrumentos relacionados acima.

\subsubsection{Critérios de elegibilidade}

Foram incluídos estudos que continham amostras composta exclusiva ou parcialmente por idosos brasileiros $\geq 60$ anos; apenas instrumentos de medida que avaliam ansiedade e/ou depressão e/ou estresse; estudos de evidências de validade ou propriedades psicométricas; publicações em português, inglês e/ou espanhol; estudos disponíveis na íntegra; e estudos publicados até 31 de dezembro de 2020.

Foram excluídos estudos que realizaram adaptação transcultural para outros países que não o Brasil; estudos de adaptação transcultural que não reportaram nenhuma evidência de validade e/ou confiabilidade; estudos em outros formatos que não artigo, como: carta ao editor, resumos, pôsteres, entre outros.

\subsubsection{Seleção dos estudos, extração dos dados e análise da qualidade metodológica}

As etapas de seleção dos estudos e extração de dados foram realizadas por dois revisores independentes que contaram com um terceiro revisor para casos de divergências. Os estudos encontrados foram extraídos da base de dados PubMed em arquivos específicos e upados no software gratuito e online Rayyan QCRI. Logo, os estudos foram analisados segundo os critérios de elegibilidade e definida a inclusão para a síntese analítica. Dos estudos finais incluídos na síntese analítica, foram extraídos dados sobre os instrumentos, população alvo e etapas de evidências e propriedades psicométricas analisadas.

Os dados foram registrados em uma planilha do Microsoft ${ }^{\circledR}$ Excel $^{\circledR}$ para Office 365 MSO desenvolvida pela autora. Para a avaliação da qualidade metodológica dos estudos, foi utilizado um protocolo adaptado com sete critérios baseados no COSMIN 2018 Risk of Bias checklist (MOKKINK, 2018). Esta adaptação foi realizada por Ferretti-Rebustini (2018) em sua Tese de Livre-Docência e parece atender mais adequadamente os fatores essenciais para avaliar a qualidade metodológica em estudos psicométricos.

Entretanto, alguns critérios também sofreram adaptação da pesquisadora principal deste estudo, visto que no estudo de Ferreti-Rebustini (2018), os critérios desenvolvidos não se estendiam à avaliação de estudos de validação subsequentes do instrumento, que são justamente os tipos de estudos contemplados neste artigo. Sofreram alterações então, os critérios C4 e C5 destacados em negrito. 
C1 - Há uma definição clara do construto a ser mensurado?

C2 - A origem do construto é clara? (há uma teoria, um modelo conceitual ou de doença usado ou foi apresentado um racional claro para definir o construto a ser mensurado?)

C3 - Foi apresentada uma definição clara do contexto em que o instrumento será usado?

C4 - Os procedimentos para as evidências de validade foram realizados em uma população representativa da população-alvo para a qual o instrumento está sendo proposto?

C5 - Foi mencionado o estudo de desenvolvimento original e o esclarecimento para qual população e contexto ele foi desenvolvido?

C6 - Foram apresentados os procedimentos de validação do instrumento?

C7 - Foram apresentados os procedimentos de análise da confiabilidade do instrumento?

Todos os estudos foram registrados em uma planilha do Microsoft ${ }^{\circledR}$ Excel $^{\circledR}$ para Office 365 MSO e analisados segundo os critérios (C1-C7) apresentados acima, por fim, foram classificados como: Adequado (descreve claramente), Aceitável (descreve parcialmente), Duvidoso (não é claro/não descreve) e Não se aplica (não se trata do objetivo do estudo).

Entretanto, foram mantidos todos os estudos que atenderam os critérios de elegibilidade, independente da qualidade metodológica, visando minimizar vieses na condução desta análise, visto que o objetivo deste estudo era investigar, as evidências de validade dos instrumentos de medida em questão. Este processo complementará a análise da suficiência destas evidências em cada um dos estudos, complementando a próxima etapa.

\subsubsection{Análise de dados}

Os dados foram extraídos, analisados e apresentados em três etapas distintas para atender os objetivos deste estudo, sendo na Etapa 1 - Síntese das evidências recuperadas (identificação do instrumento, acrônimo, variável latente, número de estudos recuperados), caracterização da amostra (quantidade de participantes, sexo, idade, faixa etária e tipo da população estudada) e caracterização dos instrumentos (objetivo do instrumento, número de fatores, número de itens, tipo de resposta, pontuação, ponto de corte e tempo de preenchimento); Etapa 2 - Análise da qualidade metodológica; cada um dos estudos foi analisado mediante aos sete critérios do protocolo determinado, e classificados como: Adequado, Aceitável, Duvidoso ou Não se aplica; Etapa 3 - Evidências de validade; para esta etapa foram definidos os critérios 
para a avaliação das evidências de validade recuperadas a fim de analisar a suficiência das evidências disponíveis.

Os resultados foram apresentados em forma de tabelas, quadros e figuras, de acordo com a melhor opção para sua representação e discutidos conforme a literatura. A validade é o conceito central da psicometria e está relacionada à interpretabilidade dos escores de um teste, conforme indicam a APA, AERA e NCME (1999), sendo que a qualidade de um teste está relacionada diretamente às suas evidências de validade. A Figura 3 demonstra a evolução do conceito de validade, entre o modelo clássico e contemporâneo.

Figura 03: Evidências de validade segundo o modelo clássico e contemporâneo, 2021

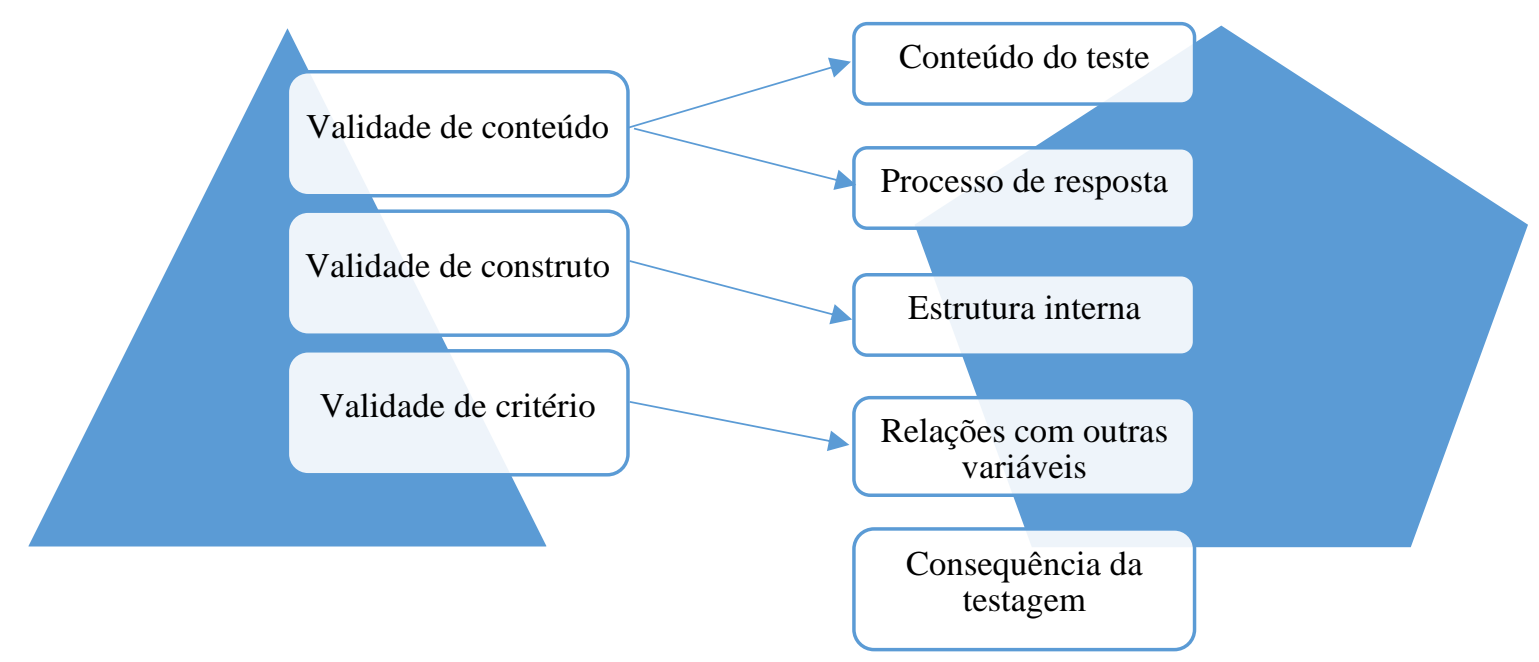

Fonte: inspirado em Primi, Muniz e Nunes (2009)

Devido a diversidade e complexidade que cada tipo de fonte de evidência de validade e a combinação de diversas técnicas qualitativas e quantitativas aplicadas a cada etapa, podem apresentar na literatura ao longo dos anos, se torna importante dar clareza ao modelo contemporâneo, possibilitando identificar corretamente nas análises, as etapas percorridas por cada estudo e os resultados atingidos de acordo com os objetivos aqui estabelecidos. O Quadro 01, proposto por Hutz (2009), apresenta esta proposta.

Quando 01: Relação de fontes de evidências de validade e procedimentos respectivos, 2021

\begin{tabular}{|l|l|}
\hline Fonte de evidência & \multicolumn{1}{c|}{ Procedimento } \\
\hline Conteúdo do teste & $\begin{array}{l}\text { Levantar dados sobre a representatividade da matriz e dos itens do teste, investigando se } \\
\text { esses consistem em amostras abrangentes do domínio que se pretende avaliar; }\end{array}$ \\
\hline
\end{tabular}




\begin{tabular}{|l|l|}
\hline $\begin{array}{l}\text { Processo de } \\
\text { resposta }\end{array}$ & $\begin{array}{l}\text { Levantar dados sobre os processos mentais envolvidos na realização das tarefas } \\
\text { propostas pela matriz; }\end{array}$ \\
\hline Estrutura interna & $\begin{array}{l}\text { Levantar dados sobre a representação do construto, com base nas dimensões avaliadas, } \\
\text { na qualidade dos itens e na confirmação de hipóteses derivadas da teoria; }\end{array}$ \\
\hline Relações com & $\begin{array}{l}\text { Levantar dados sobre os padrões de correlação entre os escores do teste e outras variáveis } \\
\text { que medem o mesmo construto ou construtos relacionados (convergência) e variáveis } \\
\text { que medem construtos diferentes (divergência). Levantar, também, dados sobre a } \\
\text { capacidade preditiva do teste com relação a outros fatos de interesse direto (critérios } \\
\text { externos) que têm importância por si só e associam-se ao propósito direto do uso do teste } \\
\text { (por exemplo, sucesso no trabalho). }\end{array}$ \\
\hline Consequências da & $\begin{array}{l}\text { Examinar as consequências sociais intencionais e não intencionais do uso do teste para } \\
\text { verificar se sua utilização está surtindo os efeitos desejados, de acordo com o propósito } \\
\text { para o qual foi criado }\end{array}$ \\
\hline
\end{tabular}

Fonte: Primi, Muniz e Nunes (2009)

Optou-se por estabelecer explicitamente os critérios e indicadores de avaliação para cada uma das fontes de evidências de validade analisadas nos estudos recuperados. O Quadro 2, apresenta detalhadamente estes conceitos.

Quadro 02: Critérios e indicadores para análise e avaliação das evidências de validade apresentadas nos estudos recuperados, 2021

\begin{tabular}{|c|c|c|}
\hline & Critérios & Indicadores \\
\hline 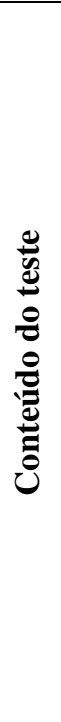 & Conteúdo do teste & $\begin{array}{l}\text { Foi aplicada algumas das recomendações para o processo de evidências, com } \\
\text { tradução e retro-tradução; } \\
\text { Foi feita análise de conteúdo por meio de um painel de especialistas adequadamente } \\
\text { composto em termos quantitativos e qualitativos; } \\
\text { Foi esclarecido como foi feita a composição do painel de especialista e suas } \\
\text { características; } \\
\text { Foram apresentados os resultados da análise de conteúdo (tabela de especificação) } \\
\text { feita por meio de um painel de especialistas e estes analisaram as evidências de que: } \\
\text { os elementos-chaves do constructo estão representados em formato de itens; os itens } \\
\text { são relevantes teoricamente, pertinentes na prática, suficientemente abrangentes no } \\
\text { conjunto e compreensíveis e representam o constructo; a organização dos itens é } \\
\text { adequada; as opções de resposta são apropriadas para o tipo de teste e estão } \\
\text { alinhadas ao conteúdo do item. } \\
\text { Foi aplicado índice de validade de conteúdo. } \\
\text { Foi aplicada uma medida objetiva de análise da validade do conteúdo e esta medida } \\
\text { é adequada para evitar inflação de resposta ou de concordância } \\
\text { Foram apresentadas as medidas tomadas a partir da análise de conteúdo (ajustes nos } \\
\text { itens) }\end{array}$ \\
\hline
\end{tabular}




\begin{tabular}{|c|c|c|c|}
\hline 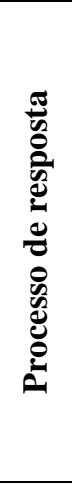 & & $\begin{array}{c}\text { Processo de } \\
\text { resposta }\end{array}$ & $\begin{array}{l}\text { Foi feita testagem na população-alvo; a amostra para a pré-testagem foi composta de } \\
\text { modo a incluir participantes da população-alvo } \\
\text { Os participantes da testagem foram questionados quando à compreensibilidade do } \\
\text { instrumento em relação às instruções de preenchimento, conteúdo dos itens e opções } \\
\text { de respostas; quanto à organização geral dos itens; quanto à abrangência (do } \\
\text { conjunto de itens) e relevância dos itens (individualmente); dificuldade de } \\
\text { preenchimento; } \\
\text { Foram realizadas entrevistas ou grupos focais } \\
\text { Foi verificado o tempo de resposta e a ausência de resposta em um ou mais itens; } \\
\text { Foi utilizado alguma técnica de acompanhamento das respostas (eye-traking) } \\
\text { Foi apresentado um mapa conceitual para a especificação de uma cadeia entre o } \\
\text { racional para responder os itens e a inferência desejada. }\end{array}$ \\
\hline \multirow{6}{*}{\multicolumn{2}{|c|}{ 苞 }} & $\begin{array}{l}\text { Intercorrelação } \\
\text { entre os itens }\end{array}$ & $\begin{array}{l}\text { Apresenta a técnica adotada: Análise Fatorial Exploratória e/ou Análise Fatorial } \\
\text { Confirmatória e/ou Teoria de Resposta ao Item e/ou Análise de Redes (Network } \\
\text { analyses). }\end{array}$ \\
\hline & & $\begin{array}{l}\text { Análise Fatorial } \\
\text { Exploratória }\end{array}$ & $\begin{array}{l}\text { O tamanho da amostra foi calculado com base no número de itens e é adequado para } \\
\text { um estudo psicométrico (5-20 representações por item, idealmente 10). } \\
\text { Há indicadores da adequação da amostra. } \\
\text { Há técnica de testagem da dimensionalidade; } \\
\text { Há especificação da técnica de extração; } \\
\text { Há especificação da técnica de rotação, quando aplicável. } \\
\text { Há técnicas e indicadores de qualidade da análise fatorial exploratória }\end{array}$ \\
\hline & & $\begin{array}{l}\text { Análise Fatorial } \\
\text { Confirmatória }\end{array}$ & $\begin{array}{l}\text { O tamanho da amostra foi calculado com base no número de itens e está adequado } \\
\text { para um estudo psicométrico } \\
\text { Há especificação da técnica de extração; } \\
\text { Foram apresentadas evidências que permitem a análise da solução fatorial com base } \\
\text { nas cargas fatoriais e } R 2 \\
\text { Foram apresentados índices de ajuste global e estatística de resíduos. } \\
\text { Foram apresentados índices de modificação e os erros das covariâncias foram } \\
\text { adequadamente manejados }\end{array}$ \\
\hline & & $\begin{array}{l}\text { Análise de } \\
\text { invariância, } \\
\text { estabilidade e } \\
\text { replicabilidade }\end{array}$ & $\begin{array}{l}\text { Foi apresentada análise de invariância métrica, configural e escalar; (AFC) } \\
\text { Foi apresentada a análise de Funcionamento diferencial do item. (TRI) } \\
\text { Foi aplicado algum índice de replicabilidade (AFE) } \\
\text { Foi realizada validação cruzada (AFE, AFC, TRI, Network) }\end{array}$ \\
\hline & & Escores & $\begin{array}{l}\text { É apresentada a forma de análise do instrumento } \\
\text { Há normatização do escores } \\
\text { Há técnica de avaliação da qualidade dos escores } \\
\text { Há técnica de classificação dos escores. }\end{array}$ \\
\hline & & Confiabilidade & Técnicas utilizadas para avaliar a confiabilidade do instrumento \\
\hline 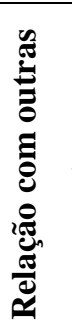 & 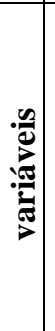 & $\begin{array}{l}\text { Procedimentos } \\
\text { aplicados visando } \\
\text { as evidências com } \\
\text { outras variáveis. }\end{array}$ & $\begin{array}{l}\text { Correlações com outros escalas ou variáveis } \\
\text { Preditivo } \\
\text { Concorrente } \\
\text { Sensibilidade e especificidade } \\
\text { Diferenças de grupo } \\
\text { Relações convergente e discriminante } \\
\text { Identificação da variância do método } \\
\text { Matrizes multitraço-multimétodo }\end{array}$ \\
\hline 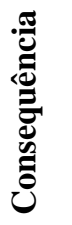 & 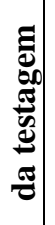 & $\begin{array}{l}\text { Consequência da } \\
\text { testagem }\end{array}$ & $\begin{array}{l}\text { Benefício previsto } \\
\text { Identificação de resultados não intencionais }\end{array}$ \\
\hline
\end{tabular}

Fonte: Aline Cristina da Silva, 2021 
Estabelecidos os critérios e seus indicadores, é possível conferir as etapas percorridas para a execução desta terceira etapa de análise de dados na Figura 4.

Figura 04: Fluxograma da terceira etapa de análise de dados dos estudos, 2021
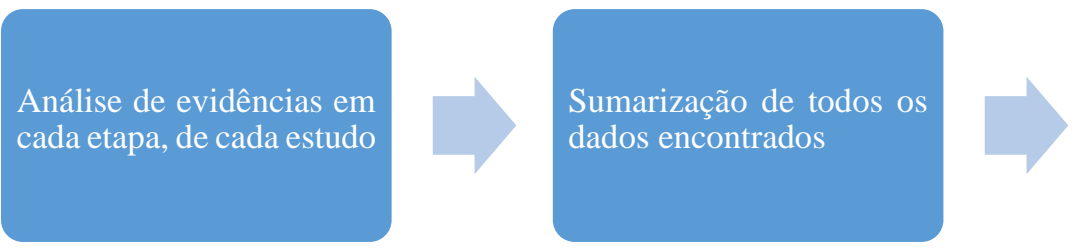

Descrição e classificação

Fonte: Aline Cristina da Silva, 2021

Como nenhuma das três etapas de análise de dados tinha caráter eliminatório, foram realizadas somente pela autora principal do artigo, sem a participação de outros revisores.

\subsection{RESULTADOS E DISCUSSÕES}

A partir das buscas na base PubMed foram recuperados 178 estudos. Foram somados a estes, 21 estudos buscados sistematicamente no primeiro artigo deste projeto e ainda, nove estudos selecionados por busca manual em artigos de interesse. Foram identificados um total de 208 estudos, sendo que 52 foram excluídos por duplicidade. Seguiram para análise de títulos e resumos, 156 artigos. Destes, foram excluídos 111, restando 45 para leitura na íntegra e análise de elegibilidade. Após a leitura completa dos artigos, foram excluídos oito estudos, por não contemplarem idosos ou idosos brasileiros em sua amostra, restando 37 estudos para a síntese analítica. A Figura 06, apresenta o fluxograma de inclusão dos estudos. 
Figura 06. Fluxograma para o processo de revisão de sistemática adaptado da declaração PRISMA, 2021

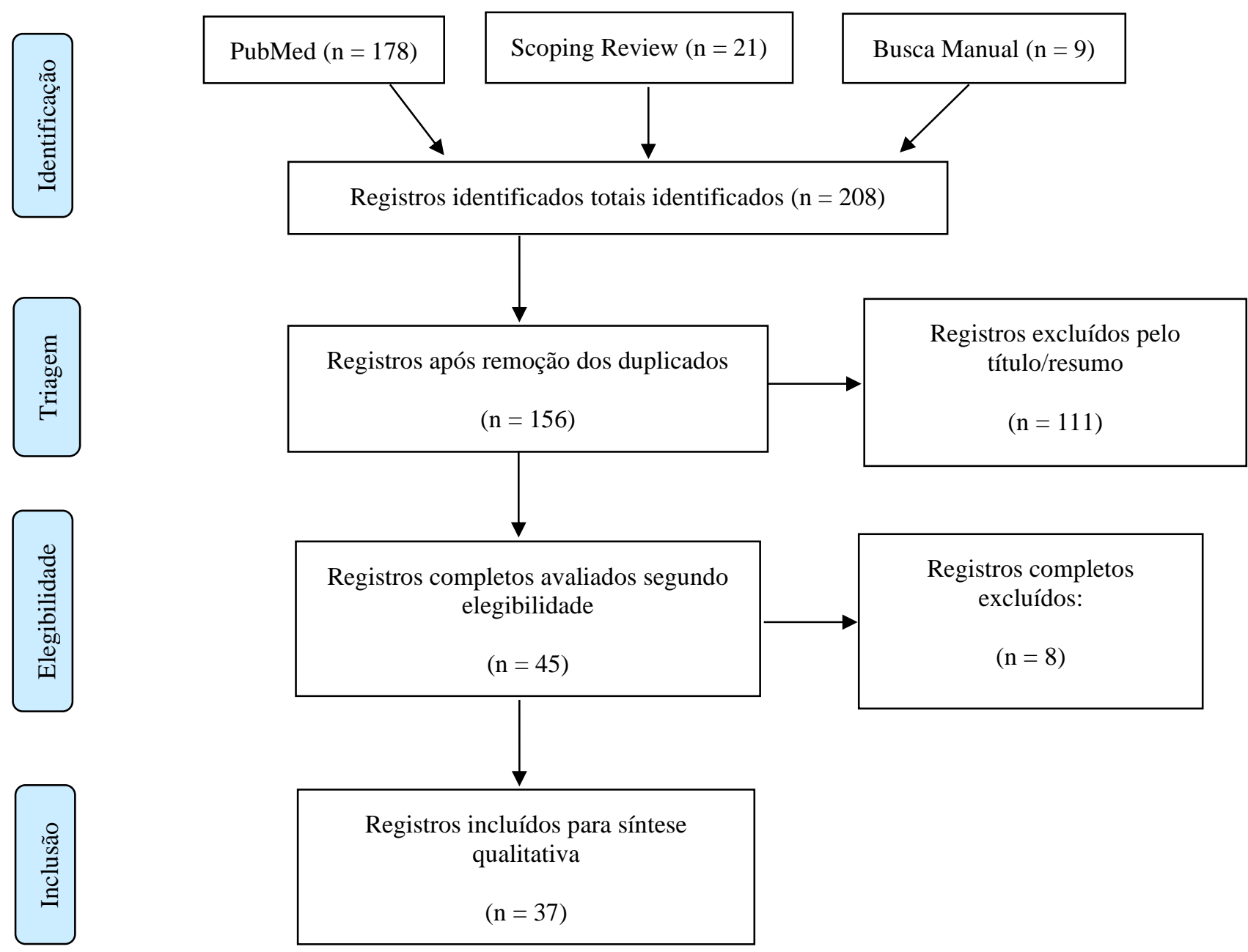

Fonte: Aline Cristina da Silva, 2021

\subsubsection{Etapa 1 - Síntese das evidências recuperadas, caracterização das amostras e dos instrumentos}

Foram identificados 25 instrumentos nos 37 estudos contemplados na síntese qualitativa, sendo que um estudo pode ter analisado evidências de mais de um instrumento. $\mathrm{O}$ Quadro 3 apresenta a relação dos instrumentos e quantidade de estudos recuperados.

Quadro 03. Frequência de estudos recuperados em relação aos instrumentos identificados, 2021

\begin{tabular}{|l|c|c|c|}
\hline \multicolumn{1}{|c|}{ Instrumento } & Acrônimo & Variável latente & $\begin{array}{c}\text { Estudos } \\
\text { recuperados } \\
\text { (N) }\end{array}$ \\
\hline Adult Stressors Inventory & & & 1 \\
\hline Baptista Depression Scale - Elderly Version & EBADEP ID & Depressão & 3 \\
\hline
\end{tabular}




\begin{tabular}{|c|c|c|c|}
\hline $\begin{array}{l}\text { Center for Epidemiologic Studies Depression } \\
\text { Scale }\end{array}$ & CES-D & Depressão & 1 \\
\hline Cornell Scale for Depression in Dementia & CSDD & Depressão & 3 \\
\hline Depression Anxiety Stress Scale & DASS & $\begin{array}{c}\text { Ansiedade, depressão } \\
\text { e estresse }\end{array}$ & 1 \\
\hline Depression Anxiety Stress Scale & DASS-Depressão & Depressão & 1 \\
\hline Depression Scale for the Elderly & DES & Depressão & 1 \\
\hline Generalized Anxiety Disorder & GAD-7 & Ansiedade & 1 \\
\hline Geriatric Anxiety Inventory & GAI-BR & Ansiedade & 1 \\
\hline Geriatric Depression Scale & GDS-1 & Depressão & 2 \\
\hline Geriatric Depression Scale & GDS-4 & Depressão & 2 \\
\hline Geriatric Depression Scale & GDS-10 & Depressão & 2 \\
\hline Geriatric Depression Scale & GDS-15 & Depressão & 8 \\
\hline Geriatric Depression Scale & GDS-30 & Depressão & 4 \\
\hline Geriatric Emotional Assessment of Pain & GEAP-b & $\begin{array}{l}\text { Depressão induzida } \\
\text { pela dor }\end{array}$ & 1 \\
\hline Hamilton Anxiety Rating Scale & HAM-A & Ansiedade & 1 \\
\hline Hamilton Depression Scale & HAM-D & Depressão & 1 \\
\hline Hospital Anxiety and Depression Scale & HADS & $\begin{array}{l}\text { Ansiedade e } \\
\text { depressão }\end{array}$ & 2 \\
\hline Liebowitz Social Anxiety Scale & LSAS & Ansiedade & 2 \\
\hline Montgomery-Åsberg Depression Rating Scale & MADRS & Depressão & 2 \\
\hline Patient Health Questionnaire & PHQ-2 & Depressão & 2 \\
\hline Patient Health Questionnaire & PHQ-9 & Depressão & 3 \\
\hline Perceived Stress Scale & PSS & Depressão & 1 \\
\hline State-Trait Anxiety Inventory & STAI & Ansiedade & 1 \\
\hline Zung Self-Rating Depression Scale & SDS & Depressão & 1 \\
\hline
\end{tabular}

Fonte: Aline Cristina da Silva, 2021

A partir deste momento, os instrumentos serão referidos por seu acrônimo. Apesar de todas as estratégias utilizadas foram identificados poucos estudos de evidências para estes instrumentos desenvolvidos ou validados para a população idosa brasileira. Poucos instrumentos foram desenvolvidos exclusivamente para idosos e este número é menor ainda quando se trata de idosos brasileiros.

O instrumento com maior quantidade de evidências recuperadas foi o GDS-15, com oito estudos, seguido do GDS-30 com 4 estudos. Na sequência, os instrumentos PHQ-9, CSDD e EBADEP-ID contaram com três estudos cada um e os instrumentos HADS, PHQ-2, ASI, LSAS, MADRS, GDS-1, GDS-4 e GDS-10 com dois estudos cada um. Os demais instrumentos, 
PSS, SDS, HAM-D, HAM-A, GAI, CES-D, STAI, DSE, DASS, DASS-Depressão, GEAP-b e GAD-7 com apenas um estudo cada. A linha temporal das evidências recuperadas se inicia em 1999 e se estende até 2020. Houve publicação em 16 dos 20 anos possíveis. O GDS é o instrumento que mais parece ter interessado os pesquisadores, sendo o mais investigado entre todos estes anos. A Tabela 08, apresenta as características das amostras envolvidas nos estudos psicométricos em relação a cada instrumento. 
Tabela 08. Características das amostras envolvidas em cada estudo psicométrico em relação a cada instrumento, 2021

\begin{tabular}{|c|c|c|c|c|c|c|}
\hline Instrumento & Autor (Ano) & $\mathbf{N}$ & $\begin{array}{l}\text { Sexo } \\
(\mathrm{M} / \mathrm{F})\end{array}$ & $\begin{array}{l}\text { Idade } \\
(\mathrm{M} \pm \mathrm{DP})\end{array}$ & Faixa Etária & Tipo da população \\
\hline ASI & Benzoni (2019) & 450 & $168 / 282$ & NI & 18 a 65 & indivíduos da comunidade \\
\hline CES-D & Batistoni et al., (2007) & 903 & $249 / 654$ & $72,4 \pm 8,3$ & 60 a 103 & idosos do projeto PENSA \\
\hline CSDD & Barca et al., (2015) & 1682 & $570 / 1112$ & $80,5 \pm 9,4$ & NI & pacientes com demência de clínicas de memória e ILPI \\
\hline CSDD & Carthery-Goulart et al., (2007) & 29 & $14 / 15$ & $74,1 \pm 8,7$ & $\mathrm{NI}$ & pacientes com DA ambulatoriais \\
\hline CSDD & Portugal et al., (2012) & 95 & $26 / 69$ & $74,4 \pm 7,7$ & NI & indivíduos com demência e depressão \\
\hline $\begin{array}{l}\text { DASS- } \\
\text { Depressão }\end{array}$ & Sardá Jr et al., (2008) & 311 & $81 / 230$ & $48,90 \pm 14,06$ & 18 a 85 & indivíduos com dor crônica \\
\hline DASS & Vignola et al., (2014) & 242 & $92 / 150$ & $39,9 \pm 12,4$ & 18 a 75 & pacientes ambulatoriais \\
\hline DSE & Giavoni et al., (2008) & 340 & $35 / 305$ & $66,81 \pm 4,71$ & 60 a 91 & idosos do projeto Geração do Ouro \\
\hline EBADEP ID & Baptista et al. (2019a) & 311 & $134 / 177$ & $70,06 \pm 7,7$ & 60 a 90 & $\begin{array}{l}\text { idosos clínicos e não clínicos (grupo de idosos, pacientes } \\
\text { internados, centro de convivência, ILPI, clínica psiquiátrica) }\end{array}$ \\
\hline EBADEP ID & Baptista et al., (2019b) & 244 & $78 / 166$ & $68,85 \pm 9,19$ & 60 a 96 & idosos da comunidade \\
\hline EBADEP ID & Coutinho et al., (2016) & 202 & $\mathrm{NI} / \mathrm{NI}$ & $68 \pm 7,22$ & 60 a 90 & idosos do centro de conveniência, ILPI e hospital \\
\hline GAD-7 & Monteiro et al., (2020) & 746 & $222 / 524$ & $23,75 \pm 8,21$ & 18 a 72 & adultos e idosos por conveniência \\
\hline GAI-BR & Massena et al., (2015) & 72 & $13 / 59$ & $72,2 \pm 6,4$ & $\geq 60$ & idosos ambulatoriais \\
\hline GDS-1 & Almeida et al., (1999) & 51 & $8 / 43$ & $67,47 \pm \mathrm{NI}$ & NI & idosos do ambulatório de saúde mental \\
\hline GDS-1 & Castelo et al., (2010) & 220 & $60 / 160$ & $\mathrm{NI}$ & 60 a 79 & idosos de uma clínica psiquiátrica \\
\hline GDS-10 & Almeida et al., (1999) & 51 & $8 / 43$ & $67,47 \pm \mathrm{NI}$ & NI & idosos do ambulatório de saúde mental \\
\hline GDS-10 & Castelo et al., (2010) & 220 & $60 / 160$ & NI & 60 a 79 & idosos da atenção primária \\
\hline GDS-15 & Almeida et al., (1999) & 51 & $8 / 43$ & $67,47 \pm \mathrm{NI}$ & NI & idosos do ambulatório de saúde mental \\
\hline GDS-15 & Alvarenga et al., (2012) & 503 & $156 / 347$ & $71,4 \pm 8,0$ & $60 \mathrm{a} \geq 70$ & idosos assistidos pela ESF \\
\hline GDS-15 & Castelo et al., (2010) & 220 & $60 / 160$ & NI & 60 a 79 & idosos da atenção primária \\
\hline
\end{tabular}




\begin{tabular}{|c|c|c|c|c|c|c|}
\hline GDS-15 & Chachamovich et al., (2010) & 424 & $152 / 272$ & $72,3 \pm 8,9$ & $60 \mathrm{a} \geq 80$ & idosos de ILPI, grupos comunitários e hospital universitário \\
\hline GDS-15 & Costa et al., (2016) & 129 & $15 / 114$ & $71 \pm 8$ & 60 a 92 & idosos por conveniência \\
\hline GDS-15 & Dias et al., (2017) & 457 & $101 / 356$ & $80,7 \pm 4,6$ & $\geq 75$ & idosos do Pietà Study \\
\hline GDS-15 & Paradela et al., (2005) & 302 & $86 / 216$ & $73,1 \pm 8,2$ & 65 a 94 & idosos ambulatoriais \\
\hline GDS-15 & Pinho et al., (2010) & 209 & $109 / 100$ & $76,54 \pm 6,72$ & $\geq 65$ & idosos com doença arterial coronariana \\
\hline GDS-30 & Castelo et al., (010)) & 220 & $60 / 160$ & NI & 60 a 79 & idosos da atenção primária \\
\hline GDS-30 & Costa et al., (2006) & 392 & $147 / 245$ & $79,9 \pm 4,5$ & $\geq 75$ & idosos do projeto BHAS \\
\hline GDS-30 & Costa et al., (2008) & 1172 & $447 / 725$ & $72,0 \pm \mathrm{NI}$ & 63 a 97 & idosos do projeto BHAS \\
\hline GDS-30 & Vargas et al., (2007) & 484 & $208 / 276$ & $70,0 \pm 7,6$ & 60 a 96 & idosos com depressão \\
\hline GDS-4 & Almeida et al., (1999) & 51 & $8 / 43$ & $67,47 \pm \mathrm{NI}$ & NI & idosos do ambulatório de saúde mental \\
\hline GDS-4 & Castelo et al., (2010) & 220 & $60 / 160$ & NI & 60 a 79 & idosos da atenção primária \\
\hline GEAP-b & Almeida et al., (2017) & 48 & $10 / 38$ & $87,5 \pm 4,1$ & 81 a 99 & idosos com dor crônica do projeto Longevos \\
\hline HADS & Faro (2015) & 690 & $304 / 386$ & $34,7 \pm 12,55$ & 18 a 65 & indivíduos estratificados por setor censitário \\
\hline HADS & Pedroso et al., (2016) & 60 & $51 / 9$ & $\begin{array}{l}61,07 \pm 5,7 \\
65,38 \pm 9,41\end{array}$ & $\geq 45$ & pacientes com AVCI de um hospital \\
\hline HAM-A & Kummer et al., (2010) & 91 & $54 / 37$ & $57,2 \pm 10,5$ & NI & indivíduos com DP ambulatoriais \\
\hline HAM-D & Costa et al., (2016) & 129 & $15 / 114$ & $71 \pm 8$ & 60 a 92 & idosos ambulatoriais \\
\hline LSAS & Kummer et al., (2008) & 90 & $54 / 36$ & $56,9 \pm 10,3$ & NI & indivíduos com DP ambulatoriais \\
\hline LSAS & Terra et al., (2006) & 300 & $275 / 25$ & $41,58 \pm 8,62$ & 20 a 60 & pacientes alcoolistas hospitalizados em clínicas psiquiátricas \\
\hline MADRS & Portugal et al., (2012) & 95 & $26 / 69$ & $74,4 \pm 7,7$ & NI & indivíduos com demência e depressão \\
\hline MADRS & Silberman et al., (2006) & 46 & $27 / 19$ & $68,1 \pm \mathrm{NI}$ & 53 a 88 & indivíduos com DP ambulatoriais \\
\hline PHQ-2 & Chagas et al., (2011) & 110 & $52 / 58$ & $61,09 \pm 12,62$ & NI & indivíduos com DP (com e sem depressão) \\
\hline PHQ-2 & Osório et al., (2012) & 100 & $61 / 39$ & $49 \pm 12,4$ & 18 a 70 & pacientes internados em enfermarias de um hospital \\
\hline PHQ-9 & Chagas et al., (2013) & 110 & $52 / 58$ & $72,3 \pm 8,9$ & NI & indivíduos com DP (com e sem depressão) \\
\hline PHQ-9 & Costa et al., (2016) & 129 & $15 / 114$ & $71 \pm 8$ & 60 a 92 & idosos ambulatoriais \\
\hline
\end{tabular}




\begin{tabular}{lllllll}
\hline PHQ-9 & Osório et al., 2012 & 100 & $61 / 39$ & $49 \pm 12,4$ & 18 a 70 & pacientes internados em enfermarias de um hospital \\
\hline PSS & Luft et al., (2007) & 76 & $6 / 70$ & $70,04 \pm 6,34$ & 60 a 84 & idosos de um grupo comunitário \\
\hline SDS & Chagas et al., (2010) & 78 & $41 / 37$ & $61,03 \pm 10,52$ & $\geq 40$ & pacientes com DP ambulatoriais \\
\hline STAI & Kaipper et al., (2010) & 900 & $190 / 710$ & $44,49 \pm 9,64$ & 18 a 60 & pacientes internados em enfermarias para cirurgia eletiva \\
\hline
\end{tabular}

NI - Não informado; AVCI - Acidente Vascular Cerebral Isquêmico; DP - doença de Parkinson; ESF - Estratégia de Saúde da Família; BHAS - Bambuí Health Aging Study; ILPI - Instituição de Longa Permanência para idosos;

Fonte: Aline Cristina da Silva 
Um total de 12.669 participantes estiveram envolvidos em todos os estudos analisados, sendo 34,33\% de homens (4.349) e 64,08\% mulheres (8.118) e 1,59\% indefinidos (202) já que a variável sexo não foi controlada. Com exceção de um estudo, todas as amostras contemplaram homens e mulheres e a quantidade amostral variou entre 29 a 1.682 participantes. O tamanho da amostra, principalmente para análises fatoriais, é um aspecto muito divergente de opiniões e controvérsias na literatura científica. Cattell (1978) argumentou que N=250 era um número minimamente recomendável, Gorsuch (1983), em consonância com Hair et al., (2005), por exemplo, recomendou que o $\mathrm{N}$ deveria ser de pelo menos 100 sujeitos, já Everitt (1975) sugeriu a presença de pelo menos 10 respostas para cada item avaliado, sendo que quanto maior o $\mathrm{N}$, melhor. O fato é que, pensando na diversidade global que estamos inseridos e somos constituídos, é quase intuitivo, compreender que uma amostra restrita, com limitação de idade, sexo/gênero, nacionalidade, escolaridade etc., pode representar o todo e generalizar a compreensão dos resultados alcançados em um único estudo.

Em relação ao tipo da amostra, 64,87\% (24) dos estudos foram realizados exclusivamente com idosos, sendo 8,33\% (2) deles com indicador cronológico para idosos a partir de 65 anos, 8,33\% (2) a partir de 75 anos, 4,16\% (1) a partir de 80 anos; e os demais estudos consideraram a partir de 60 anos. Ainda sobre a idade, 21,62\% (8) dos estudos contemplaram indivíduos a partir de 18 anos, 5,41\% (2) contaram com indivíduos a partir de 40 anos e 2,70\% (1) apenas, a partir de 50 anos. Pelo menos 21,62\% (8) dos estudos, não informaram a faixa etária da amostra participante, relatando apenas a média e desvio padrão da idade e que a amostra foi composta por adultos e idosos.

A idade cronológica é um indicador que afeta diretamente a representatividade amostral, como discutido anteriormente, paulatinamente os estudos com idosos seguem com populações de idades mais avançadas, contudo, ainda são necessários mais esforços para este avanço, estes dados reforçam a necessidade de análises com populações exclusivamente de idosos, em quantidade adequada, além das faixas etárias mais avançadas. A literatura ainda é precária para este tipo de estudo, frente a demanda demográfica e exponente crescimento desta população. Entretanto, não se pode perder de vista, como a heterogeneidade da velhice é contemplada e atendida, para além do marco cronológico (LOWSKY et al., 2014)

Vale ressaltar que a omissão da descrição, seja da quantidade de participantes, sexo, média de idade, faixa etária, enfim, ou seja, fazer bom uso da estatística descritiva é muito relevante para qualquer estudo (RODRIGUES; LIMA; BARBOSA, 2017). As medidas de tendência central (moda, mediana e média) e de dispersão ou variação (amplitude, variância, 
desvio padrão), são as mais comuns e por isso as de maior familiaridades entre todos os interessados. Organizar e descrever claramente essas e outras medidas estatísticas, se torna essencial para uma comunicação eficaz e eficiente dos dados apresentados.

Um método de pesquisa que parece contribuir com a gerontologia é o longitudinal, visto que o acompanhamento de medidas, ao longo do envelhecimento de determinadas populações e testagens em diferentes coortes, pode favorecer análises estatísticas mais robustas e completas para a compreensão de diversas características que implicam hoje uma velhice ativa e com mais qualidade. Neste sentido, foram identificados que 16,22\% (6) dos estudos aqui contemplados, tem amostras de outros estudos e projetos, como: Projeto PENSA, Projeto Geração de Ouro, Projeto BHAS, Pietà Study e Projeto Longevos.

Somente dois estudos utilizaram amostras de indivíduos com dor, sendo um somente de idosos (ALMEIDA et al., 2017), ambos tinham o objetivo de validar o instrumento para este contexto. Apenas seis estudos foram realizados em amostras com DP, sendo que nenhum deles foi somente com idosos. Somente um estudo analisou uma amostra com DA e dois estudos analisaram pacientes internados. Referente ao local, nove estudos são de amostras ambulatoriais, quatro estudos com amostra parciais provenientes de ILPI. Outros locais também foram fonte de recrutamento e demostram que em sua maioria, a população idosa é buscada em uma condição patológica ou de vulnerabilidade. Os 25 instrumentos-alvos, de avaliação de ansiedade, depressão e estresse, recuperados em cada estudo, foram apresentados em uma tabela, demonstrando suas principais características (Tabela 09). 
Tabela 09: Caracterização dos instrumentos de ansiedade, depressão e idosos apresentado em cada estudo recuperado, 2021

\begin{tabular}{|c|c|c|c|c|c|c|c|c|}
\hline Instrumento & Autor; Ano & Objetivo do instrumento & $\begin{array}{l}\text { Número } \\
\text { fatores }\end{array}$ & $\begin{array}{l}\text { Número } \\
\text { de itens }\end{array}$ & $\begin{array}{l}\text { Tipo de } \\
\text { resposta }\end{array}$ & Pontuação & Ponto de corte & $\begin{array}{c}\text { Tempo de } \\
\text { preenchimento }\end{array}$ \\
\hline ASI & Benzoni (2019) & NI & 8 & 42 & likert - 5 pts & NI & NI & NI \\
\hline CES-D & $\begin{array}{l}\text { Batistoni et al., } \\
\text { (2007) }\end{array}$ & rastreio & 3 & 20 & likert - 4 pts & 0 a 60 & $\geq 11$ = depressão & NI \\
\hline CSDD & $\begin{array}{l}\text { Barca et al., } \\
\text { (2015) }\end{array}$ & avaliar sintomas & 5 & 19 & NI & NI & NI & NI \\
\hline CSDD & $\begin{array}{l}\text { Carthery-Goulart } \\
\text { et al., (2007) }\end{array}$ & quantificar os sintomas & NI & NI & NI & NI & NI & $30^{\prime}$ \\
\hline CSDD & $\begin{array}{l}\text { Portugal et al., } \\
(2012)\end{array}$ & triagem & 4 & 19 & likert - 3 pts & 0 a 38 & 13 & NI \\
\hline DASS & $\begin{array}{l}\text { Vignola et al., } \\
\text { (2014) }\end{array}$ & avaliar sintomas & 3 & 21 & likert - 4 pts & 0 a 63 & $\begin{array}{c}\text { depressão / ansiedade } / \text { estresse } \\
\text { normal }=0-9 / 0-7 / 0-14 \\
\text { leve }=10-13 / 8-9 / 15-18 \\
\text { moderado }=14-20 / 10-14 / 19-25 \\
\text { severo }=21-27 / 15-19 / 26-33 \\
\text { muito severo }=>28 />20 />34\end{array}$ & NI \\
\hline $\begin{array}{l}\text { DASS- } \\
\text { Depressão }\end{array}$ & $\begin{array}{l}\text { Sardá Jr et al., } \\
\text { (2008) }\end{array}$ & medir sintomas & 1 & 14 & likert - 4 pts & 0 a 42 & NI & NI \\
\hline DSE & $\begin{array}{l}\text { Giavoni et al., } \\
\text { (2008) }\end{array}$ & avaliar níveis & 2 & 38 & likert - 5 pts & $\begin{array}{c}\text { média } \\
\text { aritmética } \\
\text { para cada } \\
\text { fator ( } 27 \mathrm{e} \\
11 \text { itens) }\end{array}$ & NI & $20^{\prime}$ \\
\hline
\end{tabular}




\begin{tabular}{|c|c|c|c|c|c|c|c|c|}
\hline EBADEP ID & $\begin{array}{l}\text { Baptista et al., } \\
\text { (2019a) }\end{array}$ & rastreio/detecção & 2 & 30 & dicotômica & NI & $>12$ & $30^{\prime}$ \\
\hline EBADEP ID & $\begin{array}{l}\text { Baptista et al., } \\
(2019 b)\end{array}$ & rastreio/detecção & 2 & 30 & dicotômica & NI & NI & $20^{\prime}$ \\
\hline EBADEP ID & $\begin{array}{l}\text { Coutinho et al., } \\
(2016)\end{array}$ & rastreio & NI & 45 & dicotômica & 0 a 45 & NI & NI \\
\hline GAD-7 & $\begin{array}{l}\text { Monteiro et al., } \\
(2020)\end{array}$ & rastreio & 1 & 7 & likert - 4 pts & 0 a 21 & $>8$ & NI \\
\hline GAI-BR & $\begin{array}{l}\text { Massena et al., } \\
(2015)\end{array}$ & detecção/triagem & NI & 20 & dicotômica & NI & 13 & NI \\
\hline GDS-1 & $\begin{array}{l}\text { Almeida et al., } \\
\text { (1999) }\end{array}$ & detecção & NI & 1 & dicotômica & 0 a 1 & NI & NI \\
\hline GDS-1 & $\begin{array}{l}\text { Castelo et al., } \\
\text { (2010) }\end{array}$ & triagem & 1 & 1 & dicotômica & 0 a 1 & 1 = não adequado & NI \\
\hline GDS-10 & $\begin{array}{l}\text { Almeida et al., } \\
\text { (1999) }\end{array}$ & detecção & $\mathrm{NI}$ & 10 & dicotômica & 0 a 10 & $4 / 5=$ caso/não caso & NI \\
\hline GDS-10 & $\begin{array}{l}\text { Castelo et al., } \\
(2010)\end{array}$ & triagem & 1 & 10 & dicotômica & 0 a 10 & $3 / 4=$ não caso/caso & NI \\
\hline GDS-15 & $\begin{array}{l}\text { Almeida et al., } \\
\text { (1999) }\end{array}$ & detecção & NI & 15 & dicotômica & 0 a 15 & NI & NI \\
\hline GDS-15 & $\begin{array}{l}\text { Alvarenga et al., } \\
\text { (2012) }\end{array}$ & rastreio & 4 & 15 & dicotômica & 0 a 15 & $\begin{array}{c}\text { até } 5 \text { = não caso; } \\
\geq 6=\text { caso }\end{array}$ & NI \\
\hline GDS-15 & $\begin{array}{l}\text { Castelo et al., } \\
(2010)\end{array}$ & triagem & 1 & 15 & dicotômica & 0 a 15 & $4 / 5=$ não caso/caso & NI \\
\hline
\end{tabular}




\begin{tabular}{|c|c|c|c|c|c|c|c|c|}
\hline GDS-15 & $\begin{array}{l}\text { Chachamovich et } \\
\text { al., (2010) }\end{array}$ & diagnóstico & 0 & 15 & dicotômica & NI & NI & $\mathrm{NI}$ \\
\hline GDS-15 & $\begin{array}{l}\text { Costa et al., } \\
\text { (2016) }\end{array}$ & NI & NI & 15 & NI & NI & 6 & $\mathrm{NI}$ \\
\hline GDS-15 & $\begin{array}{l}\text { Dias et al., } \\
\text { (2017) }\end{array}$ & rastreio & NI & 15 & dicotômica & 0 a 15 & $5 / 6=$ não caso/caso & NI \\
\hline GDS-15 & $\begin{array}{l}\text { Paradela et al., } \\
(2005)\end{array}$ & rastreio & NI & 15 & dicotômica & 0 a 15 & $5 / 6=$ não caso/caso & NI \\
\hline GDS-15 & $\begin{array}{l}\text { Pinho et al., } \\
\text { (2010) }\end{array}$ & rastreio & 3 & 15 & dicotômica & 0 a 15 & $5 / 6=$ não caso/caso & NI \\
\hline GDS-30 & $\begin{array}{l}\text { Castelo et al., } \\
\text { (2010) }\end{array}$ & triagem & 1 & 30 & dicotômica & 0 a 30 & $10 / 11=$ não caso/caso & NI \\
\hline GDS-30 & $\begin{array}{l}\text { Costa et al., } \\
(2006)\end{array}$ & triagem & NI & 30 & dicotômica & 0 a 30 & $14 / 15=$ não caso/caso & $40^{\prime}$ \\
\hline GDS-30 & $\begin{array}{l}\text { Costa et al., } \\
(2008)\end{array}$ & detecção/triagem & NI & 30 & dicotômica & 0 a 30 & $\begin{array}{l}10 / 11=\text { não caso/caso } \\
13 / 14=\text { não caso/caso }\end{array}$ & NI \\
\hline GDS-30 & $\begin{array}{l}\text { Vargas et al., } \\
\text { (2007) }\end{array}$ & triagem & NI & 30 & dicotômica & 0 a 30 & $\geq 12=$ depressão & NI \\
\hline GDS-4 & $\begin{array}{l}\text { Almeida et al., } \\
\text { (1999) }\end{array}$ & deteç̧ão & NI & 4 & dicotômica & 0 a 4 & NI & NI \\
\hline GDS-4 & $\begin{array}{l}\text { Castelo et al., } \\
\text { (2010) }\end{array}$ & triagem & 1 & 4 & dicotômica & 0 a 4 & $0 / 1=$ não caso $/$ caso & $\mathrm{NI}$ \\
\hline GEAP-b & $\begin{array}{l}\text { Almeida et al., } \\
\text { (2017) }\end{array}$ & rastreio & 3 & 25 & dicotômica & 0 a 25 & $\begin{array}{l}0-5=\text { pouca ou nenhuma depressão } \\
\text { induzida pela dor; } \\
5-9 \text { pontos = depressão induzida por }\end{array}$ & $5^{\prime}$ \\
\hline
\end{tabular}


dor moderada;

10-mais pontos $=$ depressão severa

induzida por dor

\begin{tabular}{|c|c|c|c|c|c|c|c|c|}
\hline HADS & Faro (2015) & rastreio & 2 & 14 & likert - 4 pts & até 21 & $\begin{array}{l}\text { HADS-A = >6; } \\
\text { HADS_D }=>5\end{array}$ & NI \\
\hline HADS & $\begin{array}{l}\text { Pedroso et al., } \\
\text { (2016) }\end{array}$ & rastreio & 2 & 14 & NI & NI & $\begin{array}{c}\text { escala total }=10 \\
\text { subescala depressão }=6\end{array}$ & NI \\
\hline HAM-A & $\begin{array}{l}\text { Kummer et al., } \\
(2010)\end{array}$ & gravidade & 2 & 14 & likert - 5 pts & 0 a 52 & quanto mais alto mais grave & NI \\
\hline HAM-D & $\begin{array}{l}\text { Costa et al., } \\
(2016)\end{array}$ & NI & NI & 17 & NI & NI & 9 & NI \\
\hline LSAS & $\begin{array}{l}\text { Kummer et al., } \\
\text { (2008) }\end{array}$ & avaliar sintomas & 2 & 24 & likert - 4 pts & 0 a 72 & $41 / 42$ & NI \\
\hline LSAS & $\begin{array}{l}\text { Terra et al., } \\
\text { (2006) }\end{array}$ & rastreio & 5 & 48 & NI & NI & $\begin{array}{c}<52 \text { = fobia leve } \\
52 \text { a } 81 \text { = fobia moderada } \\
>81 \text { = fobia severa }\end{array}$ & NI \\
\hline MADRS & $\begin{array}{l}\text { Portugal et al., } \\
\text { (2012) }\end{array}$ & triagem & $\mathrm{NI}$ & 10 & likert - 7 pts & 0 a 70 & 10 & NI \\
\hline MADRS & $\begin{array}{l}\text { Silberman et al., } \\
\text { (2006) }\end{array}$ & diagnóstico & 0 & $\mathrm{NI}$ & $\mathrm{NI}$ & $\mathrm{NI}$ & 10 & NI \\
\hline PHQ-2 & $\begin{array}{l}\text { Chagas et al., } \\
\text { (2011) }\end{array}$ & triagem & NI & 2 & likert - 4 pts & 0 a 6 & 3 & NI \\
\hline PHQ-2 & $\begin{array}{l}\text { Osório et al., } \\
\text { (2012) }\end{array}$ & triagem & $\mathrm{NI}$ & 2 & likert - 4 pts & 0 a 6 & 3 & NI \\
\hline
\end{tabular}




\begin{tabular}{|c|c|c|c|c|c|c|c|c|}
\hline PHQ-9 & $\begin{array}{l}\text { Chagas et al., } \\
\text { (2013) }\end{array}$ & triagem/diagnóstico & NI & 9 & likert - 4 pts & 0 a 27 & $\geq 9=$ depressão & NI \\
\hline PHQ-9 & $\begin{array}{l}\text { Costa et al., } \\
(2016)\end{array}$ & NI & NI & 9 & NI & NI & 10 & NI \\
\hline PHQ-9 & $\begin{array}{l}\text { Osório et al., } \\
\text { (2012) }\end{array}$ & triagem & NI & 9 & likert - 4 pts & NI & 12 & NI \\
\hline PSS & Luft et al., (2007) & grau & 2 & 14 & likert - 5 pts & 0 a 56 & $\begin{array}{c}\text { sem ponto de corte, uma vez que } \\
\text { estresse é variável contínua; }\end{array}$ & 13,8 \\
\hline SDS & $\begin{array}{l}\text { Chagas et al., } \\
\text { (2010) }\end{array}$ & rastreio & NI & 20 & likert - 4 pts & NI & $55=$ depressão & NI \\
\hline STAI & $\begin{array}{l}\text { Kaipper et al., } \\
\text { (2010) }\end{array}$ & NI & NI & 40 & likert - 4 pts & $\begin{array}{c}20 \text { a } 80 \text { para } \\
\text { cada } \\
\text { subescala }\end{array}$ & NI & NI \\
\hline
\end{tabular}

NI - Não informado

Fonte: Aline Cristina da Silva, 2021 
Em relação aos 25 instrumentos, apenas dois deles foram desenvolvidos no Brasil (ASI; DSE), sendo que apenas um deles (DSE) foi desenvolvido exclusivamente para idosos. Os demais instrumentos, com exceção do EBADEP ID, não são de origem brasileira, ou tão pouco, desenvolvidos para população idosa exclusivamente. Pode-se afirmar que a maioria dos estudos, não se preocupou em relatar detalhadamente as características dos instrumentos-alvo de análise. Das sete características buscadas para extração, referente aos instrumentos, apenas dois estudos relataram de forma completa todas elas (GEAP-b e PSS), sendo que a característica mais omitida foi o tempo de preenchimento. Não se pode afirmar que o tempo não foi controlado, entretanto, ele não foi identificado no estudo em questão. Esta variável tem grande relevância, seja para estudo ou para prática clínica, visto que instrumentos com menor tempo podem ser mais favoráveis, a depender do contexto e população, por exemplo.

Quanto ao objetivo do instrumento, a maioria relata sua finalidade como triagem, detecção e/ou rastreio da variável latente, alguns ainda utilizaram o termo diagnóstico, nível ou grau para referir a função/objetivo do instrumento. Sobre o ponto de corte do teste, este pode se apresentar diferentemente em cada estudo, mesmo para os mesmos instrumentos. Para esta definição são consideradas as medidas de sensibilidade e especificidade, lembrando que o “caso/não caso" deve ser observado com cautela, visto que variáveis contínuas por exemplo, não possuem uma separação clara do que é "normal" e "não normal” (EUGÊNIO, 2017). É importante destacar que o processo de avaliação, neste caso do idoso, deve ser mais do que uma testagem e deve contar com a expertise do profissional para um olhar mais ampliado e assertivo quanto a presença/ausência do transtorno em questão e o que isso representa para aquele indivíduo ou grupo. A descrição adequada das características do instrumento, pode facilitar a compreensão do estudo e favorecer a sua escolha para aplicação, principalmente aos profissionais que atuam com esta população e buscam na literatura evidências auxílio para sua decisão. Na próxima etapa de análise, foi verificada a qualidade metodológica de cada estudo.

\subsubsection{Etapa 2 - Qualidade metodológica}

A etapa de qualidade metodológica classifica cada estudo quanto aos procedimentos contemplados. Apesar de mais generalizada, esta análise já contribui com a terceira etapa, aonde as etapas de cada evidência proposta, foram buscadas mais detalhadamente. O quadro 04, classifica de maneira ilustrativa os estudos, segundo os sete critérios propostos no protocolo adotado para este estudo. 
Quadro 04: Relação dos estudos recuperados em relação a análise da qualidade metodológica segundo o protocolo adaptado COSMIN, 2021

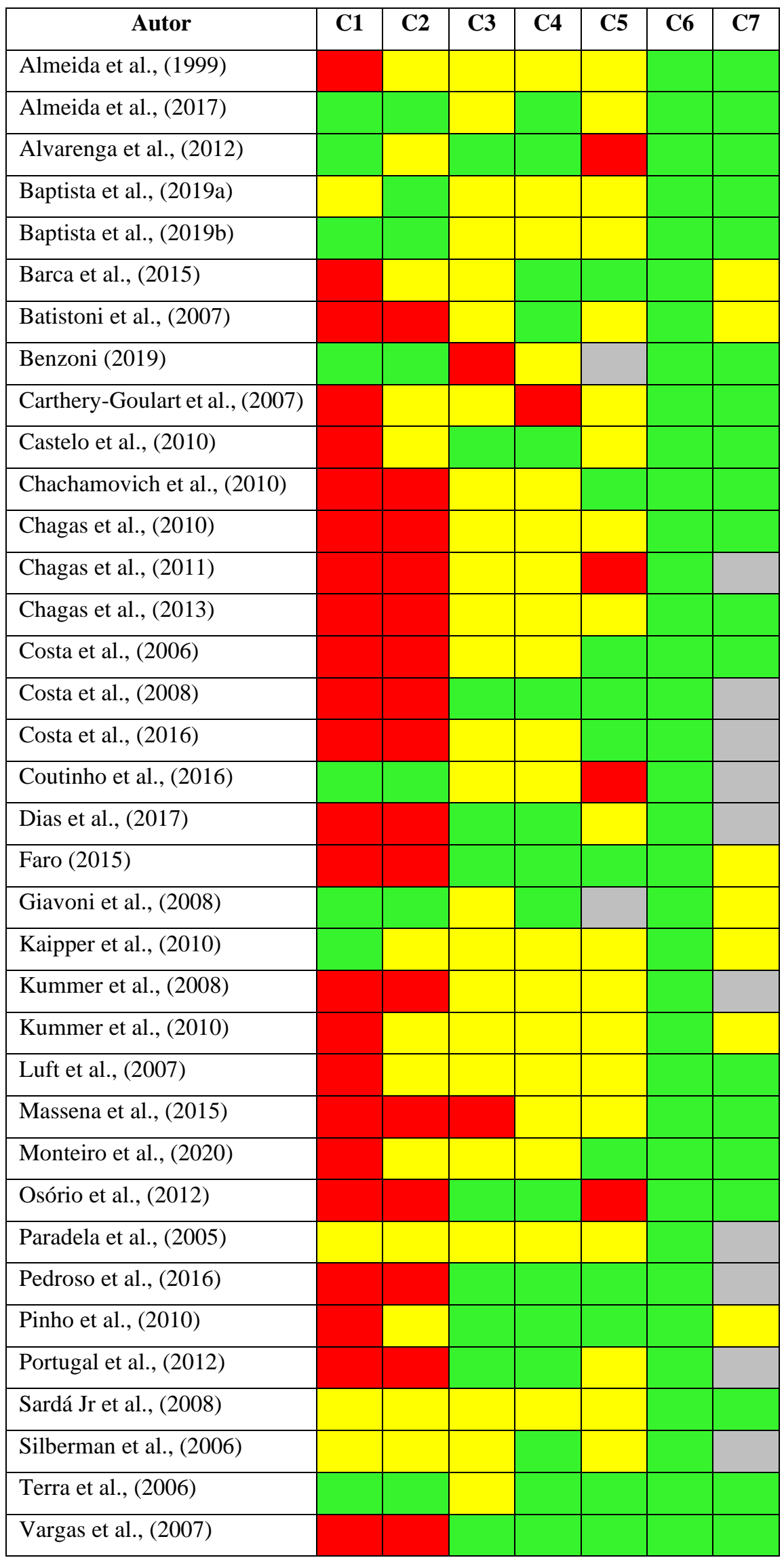




\begin{abstract}
Vignola et al., (2014)
Adequado; Aceitável, Duvidoso; Não se aplica; C1 - Há uma definição clara do construto a ser mensurado?; C2 - A origem do construto é clara? (há uma teoria, um modelo conceitual ou de doença usado ou foi apresentado um racional claro para definir o construto a ser mensurado?); C3 - Foi apresentada uma definição clara do contexto em que o instrumento será usado?; C4 - Os procedimentos para as evidências de validade foram realizados em uma população representativa da população-alvo para a qual o instrumento está sendo proposto?; C5 - Foi mencionado o estudo de desenvolvimento original e o esclarecimento para qual população e contexto ele foi desenvolvido?; C6 Foram apresentados os procedimentos de validação do instrumento?; C7 - Foram apresentados os procedimentos de análise da confiabilidade do instrumento?
\end{abstract}

Fonte: Aline Cristina da Silva, 2021

Em relação à análise metodológica, nenhum estudo apresentou todos os critérios como "Adequado". A definição do construto foi discretamente explorada, sendo considerada “Adequado" e "Aceitável” em 35\% dos estudos, já o referencial teórico/modelo conceitual foram atendidos completa ou parcialmente em 57\% dos estudos. Em 95\% dos estudos foi relatado, claramente ou parcialmente, o contexto de testagem do instrumento em questão e em 97\% dos estudos, foi utilizada uma população representativa para qual o uso do instrumento está sendo proposto. Em $84 \%$ dos estudos, foi citado o instrumento original e esclarecida a população para qual foi desenvolvido e $5 \%$ se tratava dos próprios estudos de desenvolvimento. Quanto aos procedimentos psicométricos, todos os estudos apresentaram suas descrições sobre as evidências de validade do instrumento, sendo inclusive este, um critério de elegibilidade para inclusão na síntese analítica.

Independente da classificação alcançada nesta etapa de análise, nenhum estudo foi excluído, visto que esta etapa não se tratou de uma análise pormenorizada destas evidências, logo, os 37 estudos / 25 instrumentos, seguiram para a próxima etapa de análise. Entretanto, pode-se afirmar que existem oportunidades de uma redação mais clara e objetiva para que estes tipos de estudos, possam atender as exigências mínimas dos procedimentos metodológicos de um estudo psicométrico, garantindo mais qualidade, compreensão e reprodutibilidade a todos os interessados. O Quadro 5 apresenta a sumarização das etapas realizadas em cada estudo para cada instrumento. 


\subsubsection{Etapa 3 - Evidências de validade}

Quadro 5: Evidências de validade dos instrumentos de ansiedade, depressão e estresse, 2021

\begin{tabular}{|c|c|c|c|c|c|c|c|c|c|c|c|c|c|c|c|c|c|c|c|c|c|c|c|c|c|c|}
\hline \multirow{3}{*}{ 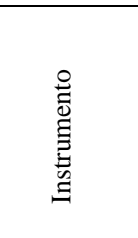 } & \multirow{3}{*}{ Autor } & \multicolumn{25}{|c|}{ Tipo de evidência } \\
\hline & & \multicolumn{4}{|c|}{ Conteúdo } & \multicolumn{5}{|c|}{ Processo de Resposta } & \multicolumn{7}{|c|}{ Estrutura Interna } & \multicolumn{7}{|c|}{ Relação com Outras Variáveis } & \multicolumn{2}{|c|}{$\begin{array}{l}\text { Consequência } \\
\text { da Testagem }\end{array}$} \\
\hline & & ADP & $\mathrm{CE}$ & IVC & IC & $\begin{array}{c}\text { PRÉ- } \\
\mathrm{T}\end{array}$ & ENT & G & $\mathrm{Tl}$ & & $\mathrm{AM}$ & DM & EX & RT & $\mathrm{Cl}$ & & ES & C_OM & $\mathrm{P}$ & $\mathrm{CO}$ & $\mathrm{COV}$ & DIS & SS & DG & $\mathrm{BP}$ & IRNI \\
\hline ASI & $\begin{array}{l}\text { Almeida et } \\
\text { al., (1999) }\end{array}$ & $*$ & 1 & 1 & 1 & 3 & 3 & 1 & 1 & & 2 & 2 & 3 & 3 & 3 & & 1 & 1 & 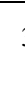 & 1 & 1 & 1 & 1 & 1 & $*$ & * \\
\hline CES-D & $\begin{array}{l}\text { Almeida et } \\
\text { al., (2017) }\end{array}$ & $*$ & * & $*$ & * & $*$ & * & * & * & & 1 & 2 & 3 & 3 & 3 & & 1 & $*$ & 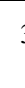 & 3 & 1 & 1 & 3 & 1 & * & $*$ \\
\hline CSDD & $\begin{array}{c}\text { Alvarenga } \\
\text { et al., } \\
\text { (2012) }\end{array}$ & $*$ & * & $*$ & * & * & * & * & * & & 1 & 2 & 3 & 3 & 2 & & 1 & $*$ & 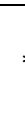 & $*$ & * & $*$ & $*$ & $*$ & * & * \\
\hline CSDD & $\begin{array}{l}\text { Baptista et } \\
\text { al., (2019a) }\end{array}$ & 3 & 3 & 2 & 3 & 3 & 1 & 1 & 1 & & 1 & 1 & 1 & 1 & 3 & & 1 & $*$ & 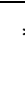 & $*$ & $*$ & $*$ & $*$ & $*$ & $*$ & $*$ \\
\hline CSDD & $\begin{array}{l}\text { Baptista et } \\
\text { al., (2019b) }\end{array}$ & $*$ & * & * & * & * & * & * & $*$ & & 1 & 1 & 1 & 1 & 2 & & 1 & 1 & i & 3 & 1 & 1 & 3 & 1 & $*$ & $*$ \\
\hline DASS & $\begin{array}{c}\text { Barca et al., } \\
\text { (2015) }\end{array}$ & 3 & 2 & 1 & 1 & 3 & 1 & 3 & 1 & & 1 & 1 & 2 & 2 & 2 & & 1 & $*$ & 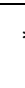 & $*$ & $*$ & $*$ & $*$ & $*$ & $*$ & $*$ \\
\hline $\begin{array}{c}\text { DASS- } \\
\text { Depressão }\end{array}$ & $\begin{array}{l}\text { Batistoni et } \\
\text { al., (2007) }\end{array}$ & 3 & 1 & 1 & 2 & 1 & 1 & 1 & 1 & & 1 & 2 & 3 & 3 & 3 & & 1 & 1 & 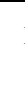 & 1 & 3 & 1 & 1 & 3 & $*$ & $*$ \\
\hline DSE & $\begin{array}{c}\text { Benzoni, } \\
\text { (2019) }\end{array}$ & $*$ & 3 & 2 & 3 & 1 & 1 & 2 & 1 & & 1 & 2 & 2 & 3 & 2 & & 3 & 1 & 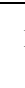 & 1 & 3 & 1 & 1 & 1 & * & $*$ \\
\hline $\begin{array}{c}\text { EBADEP } \\
\text { ID }\end{array}$ & $\begin{array}{l}\text { Carthery- } \\
\text { Goulart et } \\
\text { al., (2007) }\end{array}$ & $*$ & $*$ & * & $*$ & * & $*$ & * & $*$ & & 1 & 1 & 2 & 3 & 3 & & 1 & 1 & 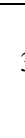 & 1 & 1 & 1 & 3 & 3 & $*$ & $*$ \\
\hline $\begin{array}{c}\text { EBADEP } \\
\text { ID }\end{array}$ & $\begin{array}{l}\text { Castelo et } \\
\text { al., (2010) }\end{array}$ & $*$ & * & $*$ & * & $*$ & $*$ & * & $*$ & 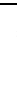 & 1 & 2 & 3 & 3 & 2 & & 1 & $*$ & 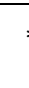 & $*$ & * & $*$ & $*$ & $*$ & * & $*$ \\
\hline
\end{tabular}




\begin{tabular}{|c|c|c|c|c|c|c|c|c|c|c|c|c|c|c|c|c|c|c|c|c|c|c|c|c|c|c|}
\hline $\begin{array}{c}\text { EBADEP } \\
\text { ID }\end{array}$ & $\begin{array}{l}\text { Chachamov } \\
\text { ich et al., } \\
\text { (2010) }\end{array}$ & $*$ & $*$ & $*$ & $*$ & $*$ & $*$ & $*$ & $*$ & * & $*$ & $*$ & $*$ & $*$ & 2 & $*$ & $*$ & 3 & 3 & 3 & 1 & 3 & 1 & 3 & $*$ & $*$ \\
\hline GAD-7 & $\begin{array}{l}\text { Chagas et } \\
\text { al., (2010) }\end{array}$ & $*$ & $*$ & $*$ & $*$ & $*$ & $*$ & $*$ & $*$ & $*$ & 1 & 3 & 3 & 1 & 3 & 1 & 1 & 3 & 3 & 1 & 3 & 1 & 3 & 3 & $*$ & $*$ \\
\hline GAI-BR & $\begin{array}{l}\text { Massena et } \\
\text { al., (2015) }\end{array}$ & $*$ & $*$ & $*$ & $*$ & $*$ & $*$ & $*$ & $*$ & $*$ & $*$ & $*$ & $*$ & $*$ & 2 & $*$ & $*$ & 3 & 3 & 3 & 1 & 3 & 3 & 3 & $*$ & $*$ \\
\hline GDS-1 & $\begin{array}{l}\text { Almeida et } \\
\text { al., (1999) }\end{array}$ & $*$ & $*$ & $*$ & $*$ & $*$ & $*$ & $*$ & $*$ & $*$ & 1 & 1 & 1 & 1 & 3 & 1 & 3 & $*$ & $*$ & $*$ & $*$ & $*$ & $*$ & $*$ & $*$ & $*$ \\
\hline GDS-1 & $\begin{array}{l}\text { Castelo et } \\
\text { al., (2010) }\end{array}$ & $*$ & $*$ & $*$ & $*$ & $*$ & $*$ & $*$ & $*$ & $*$ & 3 & 1 & 1 & 1 & 2 & $*$ & $*$ & 1 & 3 & 1 & 1 & 1 & 3 & 1 & $*$ & $*$ \\
\hline GDS-10 & $\begin{array}{l}\text { Almeida et } \\
\text { al., (1999) }\end{array}$ & $*$ & $*$ & $*$ & $*$ & $*$ & $*$ & $*$ & $*$ & $*$ & 1 & 1 & 1 & 1 & 3 & 1 & 3 & $*$ & $*$ & $*$ & $*$ & $*$ & $*$ & $*$ & $*$ & $*$ \\
\hline GDS-10 & $\begin{array}{l}\text { Castelo et } \\
\text { al., (2010) }\end{array}$ & $*$ & $*$ & $*$ & $*$ & $*$ & $*$ & $*$ & $*$ & $*$ & 3 & 1 & 1 & 1 & 2 & $*$ & $*$ & 1 & 3 & 1 & 1 & 1 & 3 & 1 & $*$ & $*$ \\
\hline GDS-15 & $\begin{array}{l}\text { Almeida et } \\
\text { al., (1999) }\end{array}$ & $*$ & $*$ & $*$ & $*$ & $*$ & $*$ & $*$ & $*$ & $*$ & 1 & 1 & 1 & 1 & 3 & 1 & 3 & $*$ & $*$ & $*$ & $*$ & $*$ & $*$ & $*$ & $*$ & $*$ \\
\hline GDS-15 & $\begin{array}{c}\text { Alvarenga } \\
\text { et al., } \\
\text { (2012) }\end{array}$ & $*$ & $*$ & $*$ & $*$ & $*$ & $*$ & $*$ & $*$ & $*$ & 3 & 2 & 3 & 3 & 3 & 1 & 3 & $*$ & $*$ & $*$ & $*$ & $*$ & $*$ & $*$ & $*$ & $*$ \\
\hline GDS-15 & $\begin{array}{l}\text { Castelo et } \\
\text { al., (2010) }\end{array}$ & $*$ & $*$ & $*$ & * & * & $*$ & $*$ & $*$ & $*$ & 3 & 1 & 1 & 1 & 2 & $*$ & $*$ & 1 & 3 & 1 & 1 & 1 & 3 & 1 & $*$ & $*$ \\
\hline GDS-15 & $\begin{array}{l}\text { Chachamov } \\
\text { ich et al., } \\
\text { (2010) }\end{array}$ & $*$ & * & $*$ & * & $*$ & $*$ & $*$ & $*$ & $*$ & 2 & 2 & 2 & 2 & 3 & 3 & 1 & $*$ & $*$ & $*$ & $*$ & $*$ & $*$ & $*$ & $*$ & $*$ \\
\hline GDS-15 & $\begin{array}{c}\text { Costa et al., } \\
\text { (2016) }\end{array}$ & $*$ & $*$ & $*$ & $*$ & $*$ & $*$ & $*$ & $*$ & $*$ & $*$ & $*$ & $*$ & $*$ & $*$ & $*$ & $*$ & 1 & 3 & 1 & 1 & 1 & 3 & 3 & $*$ & $*$ \\
\hline GDS-15 & $\begin{array}{c}\text { Dias et al., } \\
\text { (2017) }\end{array}$ & $*$ & $*$ & $*$ & $*$ & $*$ & $*$ & $*$ & $*$ & $*$ & $*$ & $*$ & $*$ & $*$ & $*$ & $*$ & $*$ & 1 & 3 & 1 & 1 & 1 & 3 & 1 & $*$ & $*$ \\
\hline GDS-15 & $\begin{array}{l}\text { Paradela et } \\
\text { al., (2005) }\end{array}$ & $*$ & * & $*$ & * & * & $*$ & $*$ & $*$ & $*$ & $*$ & $*$ & * & * & $*$ & $*$ & $*$ & 1 & 3 & 1 & 1 & 1 & 3 & 1 & $*$ & $*$ \\
\hline
\end{tabular}




\begin{tabular}{|c|c|c|c|c|c|c|c|c|c|c|c|c|c|c|c|c|c|c|c|c|c|c|c|c|c|c|}
\hline GDS-15 & $\begin{array}{l}\text { Pinho et al., } \\
\text { (2010) }\end{array}$ & $*$ & $*$ & $*$ & * & $*$ & * & $*$ & $*$ & $*$ & 1 & 3 & 3 & 3 & 3 & 1 & 1 & 1 & 3 & 3 & 1 & 1 & 3 & 3 & * & $*$ \\
\hline GDS-30 & $\begin{array}{l}\text { Castelo et } \\
\text { al., (2010) }\end{array}$ & $*$ & $*$ & $*$ & * & * & * & $*$ & $*$ & $*$ & 3 & 1 & 1 & 1 & 2 & $*$ & $*$ & 1 & 3 & 1 & 1 & 1 & 3 & 1 & $*$ & $*$ \\
\hline GDS-30 & $\begin{array}{c}\text { Costa et al., } \\
(2006)\end{array}$ & $*$ & $*$ & $*$ & $*$ & $*$ & $*$ & $*$ & $*$ & $*$ & $*$ & $*$ & $*$ & $*$ & 2 & $*$ & $*$ & 1 & 3 & 1 & 1 & 1 & 3 & 1 & $*$ & $*$ \\
\hline GDS-30 & $\begin{array}{c}\text { Costa et al., } \\
(2008)\end{array}$ & $*$ & $*$ & $*$ & $*$ & * & $*$ & $*$ & $*$ & $*$ & $*$ & $*$ & $*$ & $*$ & * & $*$ & $*$ & 1 & 1 & 3 & 1 & 1 & 1 & 3 & $*$ & $*$ \\
\hline GDS-30 & $\begin{array}{l}\text { Vargas et } \\
\text { al., (2007) }\end{array}$ & $*$ & $*$ & $*$ & $*$ & $*$ & $*$ & $*$ & $*$ & $*$ & $*$ & $*$ & $*$ & $*$ & $*$ & $*$ & $*$ & 3 & 3 & 1 & 1 & 3 & 3 & 1 & $*$ & $*$ \\
\hline GDS-4 & $\begin{array}{l}\text { Almeida et } \\
\text { al., (1999) }\end{array}$ & $*$ & $*$ & $*$ & * & $*$ & * & $*$ & $*$ & $*$ & 1 & 1 & 1 & 1 & 3 & 1 & 3 & $*$ & $*$ & $*$ & $*$ & $*$ & $*$ & $*$ & $*$ & $*$ \\
\hline GDS-4 & $\begin{array}{l}\text { Castelo et } \\
\text { al., (2010) }\end{array}$ & $*$ & $*$ & $*$ & * & * & * & $*$ & $*$ & $*$ & 3 & 1 & 1 & 1 & 2 & $*$ & $*$ & 1 & 3 & 1 & 1 & 1 & 3 & 1 & $*$ & $*$ \\
\hline GEAP-b & $\begin{array}{l}\text { Almeida et } \\
\text { al., (2017) }\end{array}$ & 3 & 3 & 1 & 3 & 1 & 1 & 1 & 1 & 1 & 1 & 1 & 1 & 1 & 3 & 1 & 1 & 1 & 3 & 3 & 1 & 1 & 1 & 1 & $*$ & $*$ \\
\hline HADS & Faro (2015) & $*$ & $*$ & * & $*$ & $*$ & $*$ & $*$ & $*$ & * & 2 & 3 & 3 & 1 & 2 & 1 & 1 & 1 & 3 & 1 & 3 & 1 & 3 & 1 & $*$ & $*$ \\
\hline HADS & $\begin{array}{l}\text { Pedroso et } \\
\text { al., (2016) }\end{array}$ & * & $*$ & $*$ & $*$ & * & * & $*$ & $*$ & * & $*$ & * & * & $*$ & * & * & $*$ & 1 & 3 & 3 & 1 & 1 & 3 & 3 & $*$ & $*$ \\
\hline HAM-A & $\begin{array}{l}\text { Kummer et } \\
\text { al., (2010) }\end{array}$ & $*$ & $*$ & $*$ & $*$ & $*$ & * & $*$ & $*$ & $*$ & 1 & 1 & 1 & 1 & 2 & $*$ & $*$ & 3 & 3 & 3 & 1 & 1 & 3 & 3 & $*$ & $*$ \\
\hline HAM-D & $\begin{array}{l}\text { Costa et al., } \\
\text { (2016) }\end{array}$ & $*$ & $*$ & $*$ & $*$ & $*$ & $*$ & $*$ & $*$ & $*$ & $*$ & $*$ & $*$ & $*$ & $*$ & $*$ & $*$ & $*$ & 3 & $*$ & $*$ & $*$ & 3 & 3 & $*$ & $*$ \\
\hline LSAS & $\begin{array}{l}\text { Kummer et } \\
\text { al., (2008) }\end{array}$ & $*$ & * & * & $*$ & $*$ & $*$ & $*$ & $*$ & $*$ & $*$ & $*$ & $*$ & $*$ & $*$ & $*$ & $*$ & 3 & 3 & 3 & 1 & 1 & 3 & 3 & $*$ & $*$ \\
\hline LSAS & $\begin{array}{l}\text { Almeida et } \\
\text { al., (1999) }\end{array}$ & $*$ & $*$ & $*$ & $*$ & $*$ & * & $*$ & $*$ & $*$ & 1 & 2 & 2 & 3 & 2 & 1 & 1 & $*$ & $*$ & $*$ & $*$ & $*$ & $*$ & $*$ & $*$ & $*$ \\
\hline MADRS & $\begin{array}{l}\text { Almeida et } \\
\text { al., (2017) }\end{array}$ & $*$ & * & $*$ & * & * & $*$ & $*$ & $*$ & $*$ & 1 & 1 & 1 & 1 & 2 & 1 & 1 & 1 & 3 & 3 & 1 & 1 & 3 & 1 & $*$ & $*$ \\
\hline
\end{tabular}




\begin{tabular}{|c|c|c|c|c|c|c|c|c|c|c|c|c|c|c|c|c|c|c|c|c|c|c|c|c|c|c|}
\hline MADRS & $\begin{array}{c}\text { Alvarenga } \\
\text { et al., } \\
\text { (2012) }\end{array}$ & $*$ & $*$ & $*$ & $*$ & $*$ & $*$ & $*$ & $*$ & $*$ & $*$ & $*$ & $*$ & $*$ & $*$ & $*$ & $*$ & 1 & 3 & 1 & 1 & 3 & 3 & 1 & $*$ & $*$ \\
\hline PHQ-2 & $\begin{array}{l}\text { Baptista et } \\
\text { al., (2019a) }\end{array}$ & $*$ & $*$ & $*$ & $*$ & $*$ & $*$ & $*$ & $*$ & $*$ & $*$ & $*$ & $*$ & $*$ & $*$ & $*$ & $*$ & 1 & 3 & 3 & 1 & 3 & 3 & 3 & * & $*$ \\
\hline PHQ-2 & $\begin{array}{l}\text { Baptista et } \\
\text { al., (2019b) }\end{array}$ & $*$ & * & $*$ & $*$ & $*$ & $*$ & $*$ & $*$ & $*$ & * & $*$ & $*$ & $*$ & 3 & $*$ & $*$ & 1 & 3 & 1 & 1 & 1 & 3 & 1 & $*$ & * \\
\hline PHQ-9 & $\begin{array}{c}\text { Barca et al., } \\
(2015)\end{array}$ & $*$ & $*$ & $*$ & $*$ & $*$ & $*$ & $*$ & $*$ & $*$ & $*$ & $*$ & $*$ & $*$ & 2 & $*$ & $*$ & 1 & 3 & 3 & 1 & 3 & 3 & 3 & $*$ & $*$ \\
\hline PHQ-9 & $\begin{array}{l}\text { Batistoni et } \\
\text { al., (2007) }\end{array}$ & $*$ & $*$ & $*$ & $*$ & $*$ & $*$ & $*$ & $*$ & $*$ & $*$ & $*$ & $*$ & $*$ & $*$ & $*$ & $*$ & $*$ & 3 & $*$ & $*$ & $*$ & 3 & 3 & $*$ & $*$ \\
\hline PHQ-9 & $\begin{array}{c}\text { Benzoni, } \\
\text { (2019) }\end{array}$ & $*$ & $*$ & $*$ & $*$ & $*$ & $*$ & $*$ & $*$ & $*$ & $*$ & $*$ & $*$ & $*$ & 3 & $*$ & $*$ & 1 & 3 & 1 & 1 & 1 & 3 & 1 & $*$ & $*$ \\
\hline PSS & $\begin{array}{l}\text { Carthery- } \\
\text { Goulart et } \\
\text { al., (2007) }\end{array}$ & 3 & 3 & 1 & 1 & 1 & 1 & 1 & 1 & 1 & 1 & 3 & 3 & 3 & 3 & 1 & 1 & 3 & 1 & 1 & 1 & 1 & 1 & 1 & $*$ & $*$ \\
\hline SDS & $\begin{array}{l}\text { Castelo et } \\
\text { al., (2010) }\end{array}$ & $*$ & $*$ & $*$ & $*$ & $*$ & $*$ & $*$ & $*$ & $*$ & * & $*$ & $*$ & $*$ & 2 & $*$ & $*$ & 3 & 3 & 3 & 1 & 1 & 3 & 3 & $*$ & $*$ \\
\hline STAI & $\begin{array}{c}\text { Chachamov } \\
\text { ich et al., } \\
\text { (2010) }\end{array}$ & $*$ & $*$ & $*$ & $*$ & * & $*$ & $*$ & $*$ & $*$ & 1 & 2 & 3 & 3 & 2 & 1 & 1 & $*$ & $*$ & $*$ & $*$ & $*$ & $*$ & $*$ & $*$ & $*$ \\
\hline
\end{tabular}

* não se aplica; 1 = não realizado; 2 = insuficiente ou indeterminado; 3 = realizado; CONTEÚDO: ADP - Protocolo de adaptação; CE - Comitê de Especialistas; IVC - Índice de Validade de Conteúdo; IC - Índice de Concordância; PROCESSO DE RESPOSTA: PRÉ-T - Pré-teste; ENT - Entrevista; GF - Grupo Focal; TR - Tempo de Resposta; ET - Eye Tracking; ESTRUTURA INTERNA: AM - Amostra; DM - Dimensionalidade; EX - Técnica de Extração; RT - Técnica de Rotação; CF - Confiabilidade; IV - Invariância; ES - Cálculo do Escore; RELAÇÃO COM OUTRAS VARIÁVEIS: C_OM - Correlação com Outras Variáveis; PR Preditivo; CO - Concorrente; COV - Convergente; DIS - Discriminante; SS - Sensibilidade e Especificidade; DG- Diferença entre Grupos; CONSEQUÊNCIA DO TEST: BP - Benefício Previsto; IRNI - Identificação de Resultados Não Intencionais.

Fonte: Aline Cristina da Silva, 2021 
O instrumento ASI foi desenvolvido por Benzoni (2019) e neste estudo, foi relatado o seu processo de desenvolvimento e validação. Para construção dos itens, foram realizadas 20 entrevistas, adultos e idosos com sintomas de estresse, aferida por outro instrumento (PSS). Foi utilizada a técnica de análise de conteúdo flutuante (busca de significados manifestos e latentes), com buscas na literatura, chegou-se a uma categorização de seis temas e 46 itens. Foi definida uma escala do tipo likert (0 a 4). As respostas precisam ser preenchidas pensando nos últimos seis meses pelos respondentes. Foi realizado um grupo piloto para verificar a estrutura semântica, o que gerou mudanças na escala de resposta. Em relação a validação, foi realizado um estudo piloto para um pré-teste. A consistência interna se deu por alfa de Cronbach e análise fatorial exploratória, rotação Varimax e normalização Kaiser-Meyer-Olkin. É mencionada uma estimativa de 9,8 participantes por item, descrita como ideal pela literatura mencionada. Foi relatada a testagem para validade de critério, comparando o ASI ao PSS. Após a AFE, foram indicados nove fatores e quatro itens foram excluídos (devido à baixa carga fatoral), nova AFE foi rodada com a versão de 42 itens e foi identificado oito fatores. Foi realizado uma ANOVA para avaliar a variação entre os resultados gerais e dos fatores versus os quartis do PSS. O instrumento foi considerado com consistência interna adequada, demonstrando validade de construto adequado e validade discriminatória. É reconhecida a limitação para comparações entre grupos e a necessidade de mais estudos relativos a esta escala.

No estudo de Batistoni et al., (2007) foi proposto a obtenção da validade interna, de construto e critério para a escala CES-D. A consistência interna foi aferida por alfa de Cronbach, calculado para todos os itens e para cada fator. Foi utilizada curva ROC para estabelecer ponto de corte. Foi realizada a análise fatorial exploratória, rotação ortogonal pelo método varimax. Foi realizada análise de componentes principais, foram obtidos três fatores após as análises. O instrumento foi relatado como um indicador possível da presença de depressão, que precisa de outras fontes para a confirmação da sua presença ou ausência, visto que pode superestimar os resultados visto os itens somáticos.

O CSDD foi analisado por três estudos diferentes aqui recuperados. Carthery-Goulart et al., (2007) traduziu, adaptou e verificou confiabilidade entre (correlação de Spearman) e intraexaminadores (Kappa) com intervalo entre a primeira e segunda avaliação. As etapas descritas foram apontadas de acordo com um protocolo, sendo, tradução (tradução por empresa especialista, retro-tradução por dois neurologistas, divergências discutidas em painel, versão final para pré-teste) e adaptação (oito indivíduos foram analisados e seus cuidadores entrevistados). 
A versão final foi aplicada por quatro profissionais, não ficando claro quantos participantes fizeram parte da amostra total (se foram diferentes subamostras ou uma amostra subdividida). O instrumento se mostrou de rápida e fácil aplicação para paciente e cuidadores. Acredita-se que esta versão contribua com novos estudos e para projetos multicêntricos. $O$ estudo de Portugal et al., (2012) teve o objetivo de avaliar duas escalas, o CSDD e o MDRS em idosos brasileiros ambulatoriais. Para tanto, foi estimada a sensibilidade e especificidade, bem como os pontos de corte por meio da curva ROC. Os escores dos instrumentos, foram comparados com outros instrumentos. Foi também avaliada a consistência interna por meio de alfa de Cronbach. O CSSD mostrou como melhor ponto de corte 13 e não houve outras recomendações sobre o instrumento.

E por fim, o estudo de Barca et al., (2015) investigou a estrutura interna do instrumento, por meio de uma análise fatorial. O total de participantes foi dividido em duas partes para realizar AFE com uma parte e AFC com a outra metade. Para a AFE, foi considerado o melhor resultado obtido por meio da análise de componentes principais e rotação oblimin. O número de fatores foi determinado por eigenvalue e analisada a "confiabilidade" por alfa de Cronbach. Para a AFC, não foi imposta nenhuma estrutura para a matriz de correlação, os ajustes foram analisados a partir de quatro índices, sendo, RMSEA, RSMSR, TLI, CFI. O que gerou cinco subescalas. O escore total e das subescalas foram comparados entre os grupos de gravidade de demência, usando o teste de Kruskal-Wallis. Foi realizada também a correlação de Pearson entre o escore total e das subescalas, de acordo com os subtipos de demências. O fator cinco foi apontado com melhor solução para validade de face. O estudo apontou diversas limitações durante a exposição dos resultados e discussões e apresentou o modelo de cinco fatores como bem ajustado.

No estudo de Vignola et al., (2014), o DASS foi traduzido e adaptado para o português brasileiro. As etapas de tradução foram descritas segundo protocolo padronizado na literatura. Foi realizado pré-teste com seis sujeitos e mais tarde aplicada a um grupo piloto. Para consistência interna, utilizou-se alfa de Cronbach. Foi realizada "análise fatorial exploratória de componentes principais" com rotação ortogonal Varimax, posteriormente, correlação entre os itens e pontuação total de cada subescala. Por fim, o estudo indica o instrumento como confiável e válido para diagnóstico e medidas terapêuticas, com ressalva as questões culturais, que podem impactar na compreensão destes transtornos.

Já o DASS-Depressão, no estudo de Sardá Jr. Et al., (2008), examinou as propriedades psicométricas da subescala Depressão do DASS em uma população com dor crônica. 
Primeiramente foi realizada tradução e a adaptação da subescala por meio de método já reconhecido na literatura científica. A última retrotradução foi comparada com a primeira tradução e foi encontrada alta concordância entre elas. Foi avaliada confiabilidade por meio de análise de divisão pela metade (correlação de Pearson) e consistência interna (alfa de Cronbach). Foi relatada análise de validade de construto (por meio de ACP e AFE), convergente (correlação com a escala PRSS-Catastrofização), discriminante e de critério (comparação das médias entre grupos sobre situação de trabalho e outras variáveis por meio do teste ANOVA). A ACP indicou apenas um fator (confirmado pelo método scree plot), a AFE usando o método inicial de fatores principais e probabilidade máxima, com rotação obliqua (Promax) também examinou o número de fatores. $\mathrm{O}$ instrumento foi considerado uma medida forte para sintomas cognitivos/afetivos da depressão em pacientes com dor crônica.

O DSE foi elaborado e validado por Giavoni et al., (2008) para avaliar níveis de depressão na população idosa, com baixo nível de escolaridade. O modelo teórico foi elaborado em três categorias, totalizando 50 itens. O painel de juízes foi composto por quatro especialistas, onde os itens foram analisados (alguns sofreram reformulação) a partir do índice de concordância acordado (nenhum item foi excluído nesta etapa). A primeira versão foi avaliada semanticamente por uma amostra de sete mulheres. No processo de validação, a maioria dos idosos tinha baixa escolaridade e utilizaram uma escala visual para responder as perguntas feitas pela aplicadora, que capturava a resposta e traduzia para o instrumento final. Utilizou-se a ACP para verificar a fatoralidade do instrumento e a AFE para extração dos dados, utilizando o método principal Axis Factoring, rotação Oblimin. Para medir a fidedignidade/consistência interna foi usado o alfa de Cronbach. Foi correlacionado os fatores do DSE ao BDI. Para avaliar a fatoração utilizou-se os testes KMO e Bartlett, para avaliar o número de fatores utilizou-se eigenvalue e o scree plot, revelando a existência de dois fatores. Foi realizada uma regressão múltipla (stepwise) para avaliar se os fatores do instrumento prediziam o escore do BDI. O instrumento foi reconhecido para pesquisa como dentro dos padrões psicométricos, mas com oportunidades de estudos futuros para validade, preditiva e concorrente, bem como confiabilidade por teste-reteste e inter-observador.

O EBADEP ID foi analisado em três estudos capturados. Sendo que o estudo de Baptista et al., (2019b) investiga a estrutura interna da escala, da versão de 30 itens. Squares (ULS), a partir de uma matriz de correlações policóricas. Foi realizada análise paralela (que encontrou três fatores) e outros dois métodos de retenção sendo, Minimum Average Partial (MAP; que considerou dois fatores) e HULL (solução unifatorial), com a rotação Direct Oblimim e o índice 
Kaise Meyer Olkin (KMO) verificou a homogeneidade. Foi considerada mais pertinente a solução com dois fatores, totalizando 15 itens (os demais foram excluídos devido as baixas cargas fatorais) assim, o índice de ajuste Global Fit Index (GFI) e o Comparative Fit Index (CFI), alcançaram uma variância explicada de $41,17 \%$ total dos itens. A correlação entre os dois fatores foi baixa e a consistência internada analisada por alfa de Cronbach. Em complemento, também foi analisada a partir da TRI por Rasch (para análise de dificuldade dos itens), o ajuste dos itens foi avaliado segundo os itens infit e outfit (valores entre 0,60 a 1,40) e por fim foi realizada a análise fatorial confirmatória para confirmar a solução de dois fatores. Os resultados do qui-quadrado e Root Mean Square Error of Approximation (RMSEA), Comparative Fit Index (CFI) e Tucker-Lewis index (TLI) foram satisfatórios. A escala foi apresentada de maneira reduzida para atender a necessidade de rápida triagem, entretanto ressalva a oportunidade de estudos futuros de análise de viés semântico (por ter itens positivos e negativos), com maior variabilidade amostral e controle de diagnóstico de depressão. O estudo de Baptista et al., (2019a) propõem uma análise fatorial do modelo bifator da escala EBADEP ID, versão reduzida de 30 itens. Foi utilizado o Weighted Least/Squares Mean and Variance Adjusted (WLSMV) como estimador para as análises fatoriais. Foi empregada a rotação bigeomin e os dois modelos (unidimensional e bifator) foram comparados com o teste quiquadrado, índices de ajuste Root Mean Square Error of Approximation (RMSEA; desejável abaixo de 0,08), Confirmatory Fit Index (CFI; desejável acima de 0,95) e Tuker-Lewis Index (TLI; desejável acima de 0,95). Uma análise suplementar da capacidade discriminativa do instrumento foi conduzida, uma ANOVA para comparar os escores entre os grupos e teste post hoc de Tuckey. Foram utilizados os coeficientes de alfa, alfa policórico e ômega para estimar a consistência interna. A fidedignidade foi avaliada por uma curva de informação do teste. Foi analisada ainda a sensibilidade e especificidade comparando os grupos. Apesar do melhor ajuste no modelo bifatorial, poucos itens carregaram nos fatores específicos e a carga no fator geral ficou muito semelhante ao modelo unidimensional, sustentando o modelo do instrumento. Já o estudo de Coutinho et al., (2016) buscou evidências de validade baseada na relação com outras variáveis e com grupos. Comparação entre as médias dos grupos com e sem comprometimento cognitivo e entre sexo, por meio do teste t Student. Foi utilizada a ANOVA com post-hoc (teste de Tukey HSD) para comparar os grupos de idosos. Por fim, realizou-se correlação de Pearson entre as variáveis e consistência interna por meio de alfa de Cronbach. O instrumento foi descrito como promissor em relação as evidências de validade, com boas qualidades psicométricas, auxiliando profissionais de saúde na avaliação da sintomatologia depressiva. 
Porém, foram recomendados outros estudos de validade, fidedignidade, especificidade, sensibilidade e normatização de uma versão diminuída e definitiva, além da aplicação em outros contextos culturais no Brasil.

O instrumento GAD-7, no estudo de Monteiro et al., (2020), propôs reunir evidências de validade, por meio de estrutura interna, relação com outras variáveis (convergente), confiabilidade e explorar a diferenciação entre indivíduos e os diferentes níveis de ansiedade. Foi realizada uma análise fatorial exploratória para determinação da dimensionalidade. Devido à natureza ordinal da medida, utilizou-se um método polícórico para a matriz de correlação, com método de extração dos mínimos quadrados com peso diagonal robusto (RDWLS), sobre o número de fatores a ser extraído, baseou-se no método HULL. O ajuste para o modelo foi avaliado sob os indicadores, RMSEA, CFI, TLI. Em relação a unidimensionalidade foram considerados, congruência unidimensional e MIREAL, finalmente, a estabilidade da estrutura fatorial foi analisada considerando o índice H. Para as análises convergentes, foram utilizados rho de Spearman e comparação de médias para discriminação dos itens entre dois grupos, por meio do teste Man-Whitney. A confiabilidade foi indicada por meio do índice de confiabilidade composto e ômega de McDonald's. O instrumento foi considerado curto, de fácil aplicação, válido e preciso para medir transtorno de ansiedade no Brasil, sendo útil para triagem TAG.

O estudo de Massena et al., (2014) teve o objetivo de validar a versão em português do GAI-BR, estimando a consistência interna, avaliando a confiabilidade e validade concorrente. Para tanto, a normalidade dos dados foi avaliada por meio do teste Kolmogorov-Smirnov, de acordo com sua distribuição, foram usadas técnicas paramétricas e não-paramétricas. A consistência interna se deu por meio de alfa de Cronbach, confiabilidade por teste-reteste, por meio de correlação de Spearman e correlação bivariada com duas outras escalas de ansiedade e com outras variáveis. Por fim, foram comparados os escores em grupos com e sem TAG e analisada a sensibilidade e especificidade por meio da curva ROC. O instrumento foi recomendado como útil e confiável para medir ansiedade em idosos brasileiros, bem como um bom instrumento, para estudos transculturais.

O GDS-1 foi analisado por Almeida et al., (1999), referente a confiabilidade por meio de teste-reteste da versão de 15 e esta versão com 1 item, foi avaliada com baixa estabilidade, $\operatorname{logo}$ os resultados encontrados em todo estudo, demonstram muitas limitações não recomendando o uso desta versão. No estudo de Castelo et al., (2010), não foi apresentada sensibilidade, mas alta especificidade, o que gerou a reflexão de que este item, que integra todas 
as versões e merece uma observação atenta pelos aplicadores, para descartar casos de depressão maior.

O GDS-4 foi avaliado neste estudo referente sua confiabilidade com teste-reteste, os itens demonstraram baixa estabilidade individualmente e seu escore total foi significativamente diferente da avaliação inicial, além da baixa consistência interna (alfa). Por esses motivos, o uso para a prática clínica não é encorajado com esta versão (Almeida et al., 1999). Já no estudo de Castelo et al., (2010), a versão de 4 itens foi avaliada junto a quatro outras escala e apresentou especificidade relativamente baixa, alta confiabilidade de consistência interna por meio de alfa de Cronbach.

Este estudo objetivou avaliar a confiabilidade do GDS-10 por teste-reteste (incluindo as versões 1, 4 e 15 itens), aonde a versão com 15 itens foi aplicada e reaplicada entre 48 e $72 \mathrm{~h}$. Foi constatada relativa estabilidade do escore total para esta versão de 10 itens, sendo recomendado seu uso na prática clínica para detecção e monitoramento da depressão em idosos (ALMEIDA et al., 1999). O estudo de Castelo et al., (2010) avaliou não só está versão, mas também outras quatro da mesma escala e para a versão de 10 itens, apresentou especificidade relativamente baixa, alta confiabilidade de consistência interna por meio de alfa de Cronbach.

O GDS-15 foi analisado no estudo de Almeida et al., (1999), não fica claro se os autores fizeram modificações no instrumento ou utilizaram uma versão já traduzida integralmente. Foi realizado procedimento de reteste de $48 \mathrm{~h}$ a $72 \mathrm{~h}$ após entrevista inicial, utilizado o Kappa para medir concordância entre itens individuais deste instrumento, o Kappa ponderado foi utilizado como estimativa da concordância entre os escores desta versão e duas outras (10 e 4 itens), ainda em condição de teste e reteste. O teste pareado de Wilcoxon e correlação de Spearman foram utilizados para comparar os escores totais da GDS, também em condição de teste-reteste (o valor estatístico normatizado para a análise foi apresentado como "z"). Foram utilizados IC 95\% e a consistência interna foi avaliada por alfa de Cronbach. As análises indicaram que os itens desta versão apresentam baixa estabilidade, mas relativa estabilidade do escore total. Por fim, o uso do instrumento é recomendado, como um indicador relativamente estável para a prática clínica, na detecção de depressão e para o monitoramento da gravidade dos sintomas ao longo do tempo em idosos. A estrutura fatorial do GDS-15 foi verificada no estudo de Alvarenga et al., (2012). Primeiramente houve cálculo amostral, com significância de 5\% e poder do teste de $80 \%$, proporção de $\mathrm{p}=0,181$ idosos com depressão e 9,3\% de precisão, o que resultou um mínimo o número de sujeitos. Para a avaliar as diferenças entre as variáveis categóricas usou-se o teste qui-quadrado. Para determinar o número de fatores usou-se o método 
scree plot e as raízes da matriz de covariância (eigenvalue) $>1$, construindo modelos com rotação oblíqua e ortogonal, foi considerada comunalidade não inferior a 0,4. A consistência interna foi avaliada por Kuder-Richardson-20. A generalização dos dados foi testada com a divisão da amostra em duas subamostras independentes e aleatórias com 50\% dos sujeitos em cada uma delas porém, houve limitação para a generalização dos resultados, que foi atribuída à amostra ser constituída exclusivamente por idosos assistidos pela ESF o que indica a necessidade de novos estudos com análise fatorial. A versão de 15 itens com estrutura única foi descrita como adequada para o rastreamento de depressão na Atenção Básica, com atenção ao item um, pois quando respondida negativamente, atribui alta predição para a depressão. Os resultados encontrados foram considerados abaixo do preconizado na literatura e por isso o uso deve ser feito com cautela.

O estudo de Castelo et al., (2010) que também investigou outras quatro versões da mesma escala, apresentou especificidade relativamente baixa, alta confiabilidade de consistência interna por meio de alfa de Cronbach e foi descrita como útil para apoiar a identificação de depressão em idosos da atenção primária. O estudo de Chachamovich et al., (2010) objetivou avaliar o desempenho psicométrico do GDS-15, como uma escala unidimensional, testada por meio de um modelo de Rasch, compreendendo os vieses potenciais sobre idade, sexo e nível educacional (DIF), em idosos brasileiros. O modelo Rasch foi aplicado e para verificar se os dados atendiam a expectativa do modelo, foram aplicados três tipos de ajustes sendo, interação item-traço (por meio do qui-quadrado), distribuição e padronização dos itens e estatísticas individuais dos itens e pessoas (analisadas para resíduos e qui-quadrado). A consistência interna foi obtida por meio da Person Separation Index (PSI). A unidimensionalidade foi testa por análise de componentes principais, o primeiro fator foi dividido em dois subconjuntos (itens com cargas positivas e itens com cargas negativas), as estimativas foram atingidas a partir desses subgrupos de itens e um teste $t$ independente foi utilizado para detectar a unidimensionalidade. Foi apresentada uma estimativa de tamanho amostral, baseado na escala, ou seja, 40-60\% endosso para itens dicotômicos, aonde uma amostra de 108 participantes, apresenta 99\% de confiança. Após análise foi identificada a unidimensionalidade da escala, é sugerida uma redução da escala para 10 itens, sendo que nenhum problema de DIF foi encontrado nesta versão reduzida. Sugere-se que a versão 15 itens, extraída da versão 30 itens, apresenta viés cultural para amostras brasileiras. O estudo reconhece sua amostra limitada, testagem de DIF para além do proposto (sexo, idade e estado civil), correção de Bonferroni, que é relatada como conservadora. Recomendou-se mais estudos 
com esta versão de 10 itens. Foi investigada a sensibilidade e especificidade do instrumento em questão e de outros dois instrumentos de avaliação de depressão. Foram usados os testes MannWhitney ou qui-quadrado para comparação entre os grupos (deprimidos e não deprimidos) com relação a idade e sexo. Foi utilizada a curva ROC para a identificação da sensibilidade e especificidade. Foi constatada excelente precisão diagnóstica para identificação de episódios depressivos maior em idoso e reconhecido como uma boa ferramenta de triagem (COSTA et al., 2016).

O estudo de Dias et al., (2017) buscou avaliar a precisão do GDS-15 para depressão tardia em idosos mais velhos da comunidade. O coeficiente de Kappa foi utilizado para analisar a concordância entre o GDS-15 e o MINI e a sensibilidade e especificidade, foi analisado pela curva ROC. O instrumento foi considerado adequado para rastreio em idosos mais velhos com baixa escolaridade. No estudo de Paradela et al., (2005), o GDS-15 teve suas medidas avaliadas em idosos ambulatoriais, para identificação de depressão maior e/ou distimia, comparando a escala aos critérios do DSM-IV. Para a comparação concorrente, utilizou-se a consulta realizada por geriatras. Sensibilidade e especificidade foram analisadas por curva ROC e as comparações das frequências foram feitas por qui-quadrado. $\mathrm{O}$ instrumento foi considerado válido para $\mathrm{o}$ rastreamento do transtorno. Já no estudo de Pinho et al., (2010) a GDS-15 teve suas propriedades psicométricas avaliadas em portadores de DAC ambulatoriais. A consistência interna foi avaliada por Kurder-Richardson-20 e correlação dos itens com o escore total. Foi realizada análise fatorial exploratória, com matriz de correlação tetracórica, a escolha do número de fatores foi feita pelos métodos scree splot e eigenvalue. Foram construídos modelos com rotação obliqua e ortogonal (Varimax e Promax), sendo os ajustes dos modelos considerados por GFI e RMSR. Foi utilizado cálculo da AUROC com correção de viés de verificação. Correlação de Spearman ou Gamma de Goodman-Kruskal (ggk) foram usados para descrever a relação entre duas variáveis e análise de variância de Kruskal-Wallis testou igualdade das medianas entre os grupos e método de diferença mínima significante. A medida convergente se deu pela comparação com outras escalas e a medida com outras variáveis se deu com os critérios operacionais de depressão e distimia segundo o DSM-IV. O instrumento foi considerado com boa confiabilidade, boa validade concorrente e de critério para identificação de depressão em idosos com DAC. Ressalva ao item 9 que se mostrou com baixa discriminação e prejuízo em consistência interna.

O GDS-30 contou com quatro estudos, sendo que o estudo de Castelo et al., (2010) buscou determinar a validade de cinco versões desta escala (GDS-1, GDS-4, GDS-10, GDS-15 
e GDS-30) e calcular os pontos de corte para a identificação de depressão em idosos na atenção primária. Para tanto, foi estimado o tamanho da amostra com base em uma sensibilidade esperada de 0,85 (IC=0,65) assumindo uma prevalência aproximada de $20 \%$ de depressão geriátrica na atenção primária. Foi utilizada a medida clínica como padrão ouro; sensibilidade e especificidade (PPV e NPV), valores preditivos (positivo e negativo), para as diferentes pontuações de corte (considerada validade de critério), avaliado pela curva ROC e a consistência interna foi medida pelo alfa de Cronbach. A versão com 30 itens, apresentou sensibilidade e especificidade alta, além de alta confiabilidade de consistência interna por meio de alfa de Cronbach, foi indicada para uso rotineiro de triagem na atenção primária.

No estudo de Costa et al., (2006), foi medida a sensibilidade e especificidade, calculando todos os possíveis pontos de corte por meio da curva ROC. O GDS-30 foi considerado insuficientemente preditivo em relação ao diagnóstico ICD-10/SCAN para recomendação de seu uso como triagem em pesquisa. O estudo de Costa et al., (2008), retratado com inédito, investigou a concordância entre o GDS-30 e o GHQ por meio do coeficiente de Kappa. As pontuações foram obtidas para diferentes cut-offs e correções de pontuação. As diferenças calculadas via qui-quadrado para a escolaridade, sexo e idade. As taxas de prevalência de depressão foi muito acima do observado anteriormente e recomendam que novos estudos de validade sejam feitos para idosos mais velhos brasileiros. No estudo de Vargas et al., (2007), o GDS-30 foi investigado com idosos da atenção primária. As variáveis foram comparadas entre grupos pelos testes t Student ou Mann-Whitney, já as variáveis qualitativas foram comparadas pelo teste qui-quadrado ou teste exato de Fisher. A capacidade discriminativa do instrumento se deu pela análise da curva ROC. A associação da GDS ao diagnóstico pelo SCID-CV se deu por regressão logística multivariada. O instrumento foi considerado com desempenho adequado com ponto de corte 12, de fácil aplicação e útil para idosos ambulatoriais.

Neste estudo sobre o GEAP-b, objetivou-se a tradução e adaptação transcultural do instrumento, sendo mencionada a utilização de um protocolo padronizado para este procedimento, descritos completamente em cada etapa de tradução (tradutores independentes) e adaptação (equivalência semântica, idiomática, transcultural, conceitual) sendo que a etapa de equivalência transcultural/experimental foi destacada como uma evidência de validade de conteúdo. E a última etapa, equivalência conceitual, contou com a participação de um comitê de especialistas, chegando-se a uma versão final com 25 questões dicotômicas. O instrumento foi aplicado por dois inter-observadores no mesmo dia e reaplicado após 15 dias, objetivando a confiabilidade do instrumento por teste-reteste. A consistência interna se deu por alfa de 
Cronbach para os três aplicadores, a reprodutibilidade foi avaliada por três análises diferentes, entre os resultados dos três aplicadores por meio do teste $\mathrm{t}$ Student, ICC e índice de concordância de Kappa. Foi ainda aplicada correlação de Pearson comparado o instrumento a outras duas escalas. $\mathrm{O}$ instrumento foi considerado com boa/excelente consistência interna, boa reprodutibilidade, fácil aplicação e compreensão dos idosos, ótimo tempo de aplicação e por isso válido para a identificação de depressão induzida pela dor em idosos (ALMEIDA et al., (2017).

O estudo de Faro (2015) analisou o HADS, que objetivou realizar análise fatorial confirmatória e análise de curva ROC para normatização dos pontos de corte. Foi realizada a AFC com método de estimação de máxima verossimilhança, para ajuste do modelo usou-se qui-quadrado, GFI, CFI, RMSEA. Os modelos foram comparados por dois índices, critério de informação de Akaike (AIC) e critério de informação de Bayes (BIC). A confiabilidade foi calculada por alfa de Cronbach e o cálculo da validade preditiva bem como pontos de corte foram calculados por meio de curva ROC e análise de convergência com correlação de Pearson. Também teve seu desempenho avaliado pelo estudo de Pedroso et al., (2016), variáveis demográficas e clínicas foram comparadas entre os grupos (com e sem depressão) por meio do teste qui-quadrado, Mann-Whitney e correlação de Spearman. Análises de curva ROC foram realizados para identificação de pontos de corte ideais para diagnóstico de depressão. Concluise que a HADS tem bom desempenho para o rastreamento de sintomas depressivos em após AVC agudo.

O HAM-A foi analisado no estudo de Kummer et al (2010), os dados categóricos foram comparados entre os grupos (com e sem TAG) por meio do teste exato de Fisher, para variáveis contínuas usou-se o teste Mann-Whitney. A normalidade foi avaliada pelo teste de ShapiroWilk. Para as correlações foi utilizado o Rho de Spearman (entre outras escalas e outras variáveis). Buscou-se um ponto de corte adequado para discriminar pacientes com ou sem TAG, por meio de curva ROC. Por fim, se propôs avaliar a consistência interna por meio do alfa de Cronbach e correlação de Spearman. O instrumento foi recomendado como confiável para avaliar ansiedade em pacientes com DP com corte 10/11.

O HAM-D foi analisado pelo estudo de Costa et al., (2016), investigando a sensibilidade e especificidade do instrumento em questão e de outros dois instrumentos de avaliação de depressão. Foram usados os testes Mann-Whitney ou qui-quadrado para comparação entre os grupos (deprimidos e não deprimidos) com relação a idade e sexo. Foi utilizada a curva ROC para a identificação da sensibilidade e especificidade. Foi constatada excelente precisão 
diagnóstica para identificação de episódios depressivos maior em idoso. Apesar do ótimo resultado, não é recomendado como melhor opção para triagem de depressão visto outras características como ser longo e necessitar treinamento específico para aplicação e correção.

Dois estudos avaliaram o LSAS, com amostras diferentes. O estudo de Kummer (2008), objetivou explorar as propriedades psicométricas do instrumento em pacientes com DP. Foram comparadas as variáveis categóricas entre os grupos (com e sem fobia social) por meio de quiquadrado, para variáveis contínuas foi utilizado o teste t Student e distribuição não paramétrica, teste Mann-Whitnney. Foi realizada também correlação entre o LSAS, outras escalas e medida clínica, por meio da correlação de Spearman e análise de curva ROC para sensibilidade e especificidade. $\mathrm{O}$ instrumento foi relatado como projetado para dimensionar e avaliar sintomas fóbicos, mas deve ser usado cautelosamente para triagem ou diagnóstico, pois alguém com sintomas leves ou difusos, podem apresentar pontuações semelhantes a pacientes com sintomas restritivos. O estudo confirma que pacientes com DP tem sintomas frequentemente associados a outros transtornos de ansiedade e depressivos. O estudo de Terra et al., (2006) objetivou investigar a consistência interna e estrutura fatorial do LSAS. Não foi relatado cálculo amostral. A consistência interna foi avaliada por meio do alfa de Cronbach, os coeficientes de correlação item-total corrigidos foram calculados para cada item. Foi relatado a execução de análise fatorial por meio de componentes principais para explorar a dimensionalidade e rotação varimax. Por fim a escala foi considerada válida e confiável para alcoolistas.

O estudo de Silberman et al., (2006) analisou o MADRS com o objetivo de estabelecer pontos de corte para o diagnóstico clínico de depressão em DP. O MADRS foi comparado a outro instrumento via curva ROC, a capacidade de ambas escalas foi estimada pela estatística Kappa. Os grupos com e sem depressão foram comparados por meio do qui-quadrado. Foi estabelecida concordância entre medida diagnóstica (DSM-IV) e MADRS por meio de Kappa. Foi concluído que o MADRS tem boa precisão e concordância para o diagnóstico de depressão leve em paciente com DP. No estudo de Portugal et al., (2012), foi investigado por meio de uma amostra de idosos ambulatoriais, com e sem demência. Sensibilidade e especificidade se deram por meio de curva ROC, consistência interna por alfa de Cronbach e correlação entre os itens. O MADRS se correlacionou com apenas uma outra escala. O ponto de corte mais adequado foi 10 não havendo nenhuma recomendação direta para uso ou não uso do mesmo.

O PHQ-2 foi investigado por dois estudos, sendo um deles o de Osório et al., (2012), que objetivou determinar sua capacidade para avaliação de transtornos depressivos em pacientes internados, para tanto, foram utilizados curva ROC e coeficiente de Kappa para 
validade discriminante e alfa de Cronbach e ICC para análise de confiabilidade. Foram apresentados o PPV, NPV e ICR das versões 2 e 9 itens. Foi realizada correlação dos instrumentos com o diagnóstico geral pelo SCID-IV. Parece que o PHQ-2 apresentou potencial discriminante tão adequado quanto outros instrumentos, considerando o ponto de corte 3 . Tem curta aplicação e envolve baixo custo, ajudando a detecção do transtorno em ambientes hospitalares. Este também foi investigado por outro estudo (Chagas et al., 2011), com o objetivo de avaliar suas qualidades psicométricas, houve comparação entre grupos (deprimido e não deprimido) a partir do teste $\mathrm{t}$ Student para as variáveis quantitativas e qui-quadrado para variáveis categóricas. A validade concorrente foi testada por meio da correlação de Spearman, entre o PHQ-2, GDS-15 e o SDS, também com outras variáveis. Foi utilizado a curva ROC para traçar a relação de taxas de verdadeiro positivo e falso positivo para o PHQ-2 e o item de depressão da UPDRS. Foi considerado válido para triagem e discriminação de depressão maior em pacientes com DP.

O instrumento PHQ-9 foi analisado por 3 estudos. O estudo de Costa et al., (2016), investigou a sensibilidade e especificidade do PHQ-9 e de outros dois instrumentos de avaliação de depressão. Foram usados os testes Mann-Whitney ou qui-quadrado para comparação entre os grupos (deprimidos e não deprimidos) com relação a idade e sexo. Foi utilizada a curva ROC para a identificação da sensibilidade e especificidade. Foi constatada excelente precisão diagnóstica para identificação de episódios depressivos maior em idoso. Em comparação as outras duas escalas, não foram reconhecidas diferenças significativas, mas devido a outras características apontadas, o PHQ-9 foi recomendado como ferramenta mais adequada para triagem e avaliação dos sintomas depressivos em ambientes clínicos. O estudo de Chagas et al., (2013) sobre o PHQ-9, investigou evidências em relação com outras variáveis por meio de análise discriminante, consistência interna, sensibilidade e especificidade (PPV e NPV). Houve comparação entre grupos (deprimidos e não deprimidos) com os testes t Student e qui-quadrado. Foi utilizado o teste de Spearman para comparação entre o PHQ-9, GDS-15 e SDS (está análise foi denominada inicialmente no método como concorrente e como convergente na discussão). A consistência interna foi testada por alfa de Cronbach e o AUC foi usado como indicador de capacidade, diferenciando os grupos. Concluiu-se que PHQ-9 não parece eficaz para identificar depressão maior em indivíduos com DP e apresenta limitação quanto à escolaridade, por ser autorelato. Entretanto, foi compreendido como válido para triagem nesta população. O estudo de Osório et al., (2012), objetivou determinar sua capacidade para avaliação de transtornos depressivos em pacientes internados, para tanto, foram utilizados curva ROC e coeficiente de 
Kappa para validade discriminante e alfa de Cronbach e ICC para análise de confiabilidade. Foram apresentados o PPV, NPV e ICR das versões 2 e 9 itens. Foi realizada correlação dos instrumentos com o diagnóstico geral pelo SCID-IV. O ponto de corte mais adequado foi 12, sendo considerado com boas propriedades psicométricas, para uso em contexto hospitalar.

O PSS foi avaliado por um único estudo, de Luft et al., (2007), com o objetivo de tradução e validação para idosos brasileiros. A tradução foi realizada baseada em um protocolo padronizado (Guillemin et al., 1993) descrevendo a etapa de tradução e tradução reversa, bem como todos os aspectos observados pelos especialistas (equivalência semântica, idiomática, experimental ou cultural, conceitual). A validação inicial se deu em um grupo de idoso (N=76), não foi relatado cálculo amostral, com análise fatorial exploratória, utilizando a ACP para extração dos fatores, gerando uma matriz correlacional a partir da observação dos eigenvalues. Para a interpretação da matriz foi aplicado o método de rotação ortogonal Varimax. Optou-se por avaliar a consistência interna (relatada como confiabilidade) por meio do coeficiente de alfa de Cronbach e as cargas fatorais de duas versões do instrumento (10 e 14 itens). O escore do PSS foi comparado com outras variáveis coletadas por autorrelato por meio da ANOVA (mais de duas possibilidades de respostas), teste t Student (até duas respostas). Na versão de 10 itens, o fator 1 apresentou cargas fatorais mais altas, com 42,5\% de variância explicada e aumento da consistência interna (alfa). O estudo relata que não apresenta ponto de corte para o instrumento, visto que o estresse é uma variável contínua, portanto, estas perdem sensibilidades quando classificadas dessa forma. Os autores considerados o PSS com características psicométricas adequadas para validade de construto e consistência interna, recomendando o uso do instrumento para idosos brasileiros em estudos epidemiológicos e multiculturais.

O teste SDS foi avaliado apenas por um estudo (Chagas et al., 2010), com o objetivo de validá-lo para triagem de pacientes com DP. Os dados entre os grupos, com e sem depressão, foram comparados a partir do teste t Student, para as seguintes variáveis: diagnóstico de depressão, idade, distribuição de sexo, idade no início da doença e duração da doença. Foi aplicado o teste de correlação de Spearman para avaliar a correlação com outras variáveis como, GDS-15, escore motor, escalas HY, SE (medidas convergentes/concorrentes e diretas). Foram também analisados os valores de corte por Curva ROC, a Consistência Interna foi relatada por meio de testagem de alfa de Cronbach e correlação item-pontuação total (essas testagens foram feitas com diferentes versões reduzidas e alcançaram diferentes resultados). Por fim, a escala foi recomendada como útil, para rastreio de depressão em pacientes com DP com índice de 55, entretanto foi relatado a sobreposição de sintomas entre os itens de depressão e DP, bem como 
outras necessidades a serem discutidas, como estudos com outras populações de DP e amostras geriátricas exclusivas.

Apenas um estudo avaliou o STAI, com o objetivo de testar estrutura interna, a partir de um modelo da Teoria de Resposta ao Item (TRI), o modelo de Rasch. Para aplicação deste modelo, é essencial que o teste seja unidimensional, ou seja, que os itens do teste meçam apenas um fator ou construto, por isso, a unidimensionalidade do instrumento foi avaliada por duas estratégias, Análise de Componentes Principais com Rotação Varimax com todos os itens (este procedimento foi chamado de Análise Fatorial), corrigido pela Análise Paralela de Monte Carl. Posteriormente, foram analisados os resíduos dentro do modelo de Rasch, o primeiro fator da ACP foi dividido em dois subconjuntos (itens mais positivos e itens mais negativos) e a estimativa de pessoas foi feita a partir destes dois subconjuntos de itens, e um Teste $\mathrm{t}$ independente, foi usado para detectar as diferenças significativas, que apontaram a unidimensionalidade para cada subescala. A interação item-traço foi analisada via quiquadrado, indicando a invariância (modelo esperado e modelo observado). Uma estimativa de consistência também foi obtida pela pelo índice de separação de pessoas (PSI), equivalente ao coeficiente de Alfa de Cronbach. Apesar de discutir a importância da questão amostral, o estudo não apresentou cálculo da sua amostra. Foram declarados 900 participantes e um análise com um subgrupo de 70 participantes. Algumas retestagens foram feitas com amostras aleatórias de 300 participantes. Após as análises, foi considerada a proposta de uma versão mais curta e mais consistente do instrumento STAI-Form X, descrita como mais útil para avaliar ansiedade nos diferentes contextos clínicos (KAIPPER et al., 2010). A seguir, é possível verificar quais foram as fontes de evidências de validade mais analisadas pelos autores dos estudos recuperados (Figura 07). 


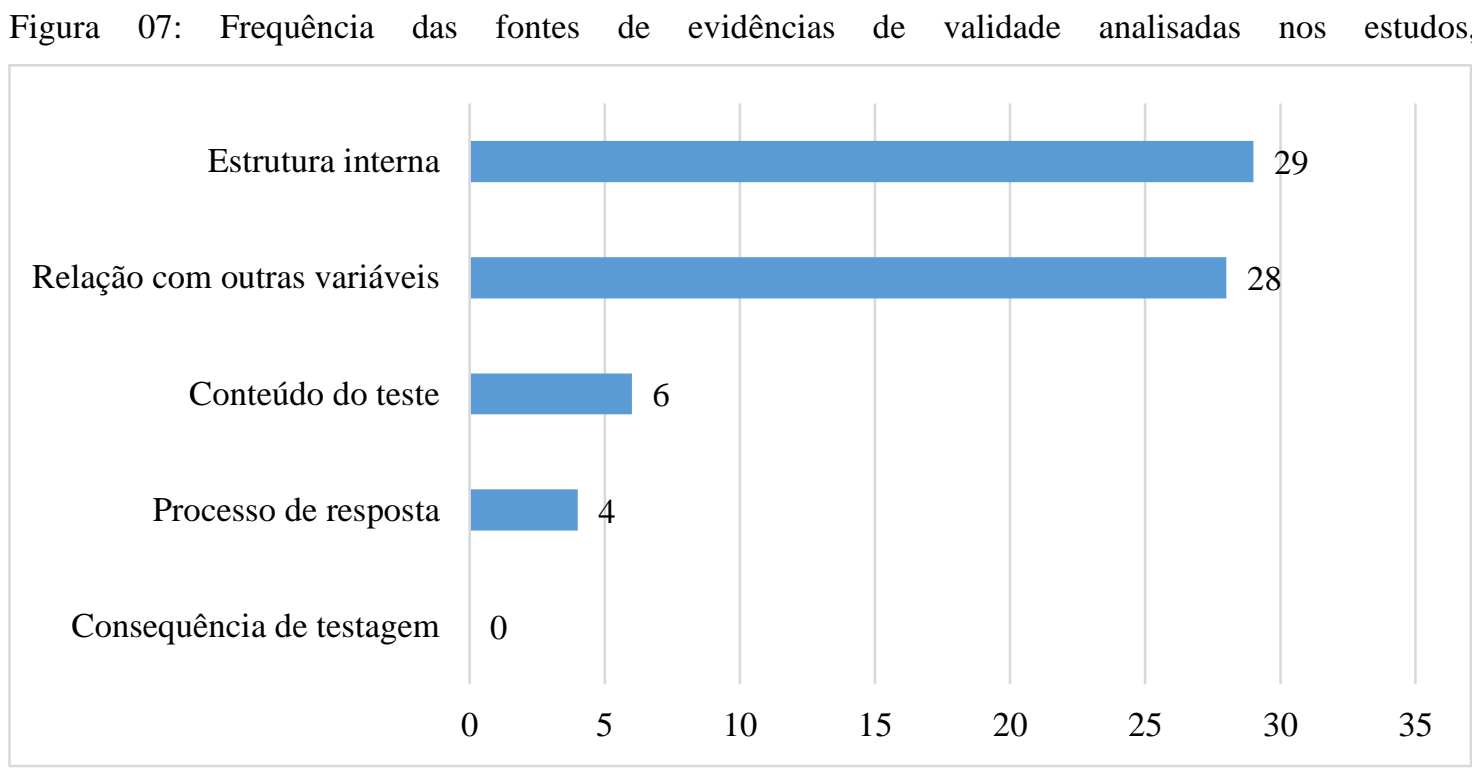

Fonte: Aline Cristina da Silva, 2021

Os tipos de fontes de evidências mais explorados pelos autores, foram análises de estrutura interna, seguida por relação com outras variáveis. Sendo que de acordo com os critérios estabelecidos para este estudo, os procedimentos mais recorrentes foram as análises fatoriais (exploratória, confirmatória e análise de componentes principais), análises bifator e análises para confiabilidade, por meio de consistência interna, com os testes de alfa de Cronbach, K-20 e teste e reteste.

Vale destacar que apenas seis estudos descreveram os critérios para definição/cálculo da amostra participante no estudo. Referente aos procedimentos para as evidências de relação com outras variáveis, os mais predominantes foram análises preditivas de curva ROC, para identificação da sensibilidade e especificidade do instrumento, bem como ponto de corte para a população específica e versão estudada.

Outros critérios muito presentes, foram a avaliação concorrente, por meio de correlações com outros instrumentos e as comparações dos escores entre grupos. Todos os critérios estabelecidos aqui, para esta fonte de evidência, foram explorados em pelo menos um estudo. Foram contemplados no gráfico abaixo (Figura 08) apenas instrumentos com três ou mais fontes de evidências de validade. 
Figura 08: Instrumentos com maior quantidade de fontes de evidências avaliadas, 2021

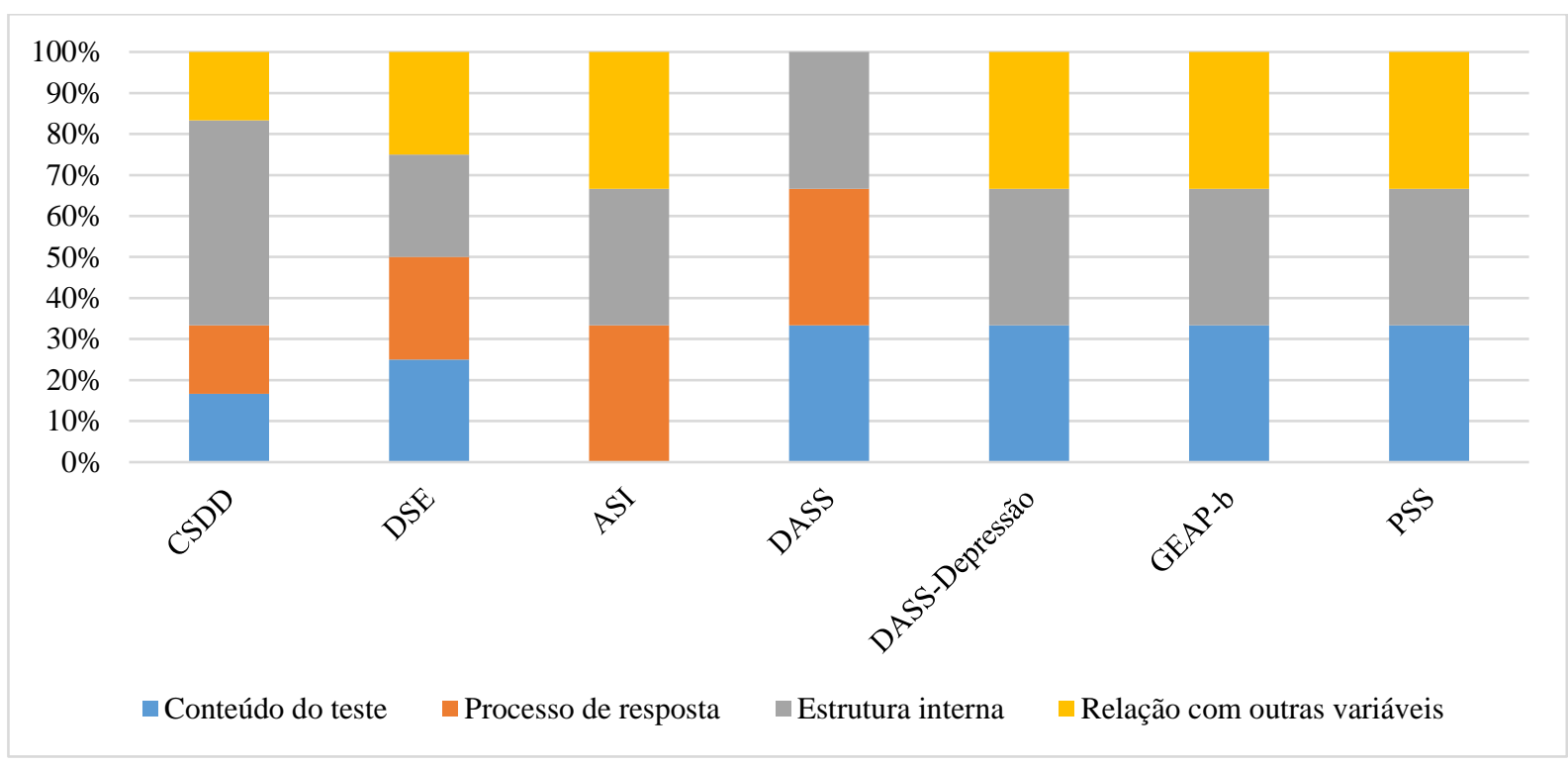

Fonte: Aline Cristina da Silva, 2021

Os instrumentos com mais variabilidade entre as fontes de evidência de validade foram o CSDD, DSE, ASI, DASS, DASS-Depressão, GEAP-b e PSS.

O CSSD foi avaliado por três estudos, já o DSE, ASI, DASS, DASS-Depressão, GEAPb e PSS foram analisados em apenas um estudo. Todos estes estudos contaram com procedimentos de desenvolvimento ou tradução e adaptação do instrumento procedimento de evidências de conteúdo, parece que estes tipos de estudos, também são mais propensos a estender as análises buscando o máximo de fontes de evidências de validade possível. Os procedimentos ligados as evidências de processo de respostas, estão respectivamente ligados a estudos de desenvolvimento/tradução e adaptação também, logo, concentrados neste grupo. Entretanto, foi buscado por quatro dos sete instrumentos deste grupo. Na figura abaixo, é possível verificar os instrumentos que reuniram um ou dois tipos de evidências de validade (Figura 09). 
Figura 09: Instrumentos com maior quantidade de fontes de evidências avaliadas, 2021

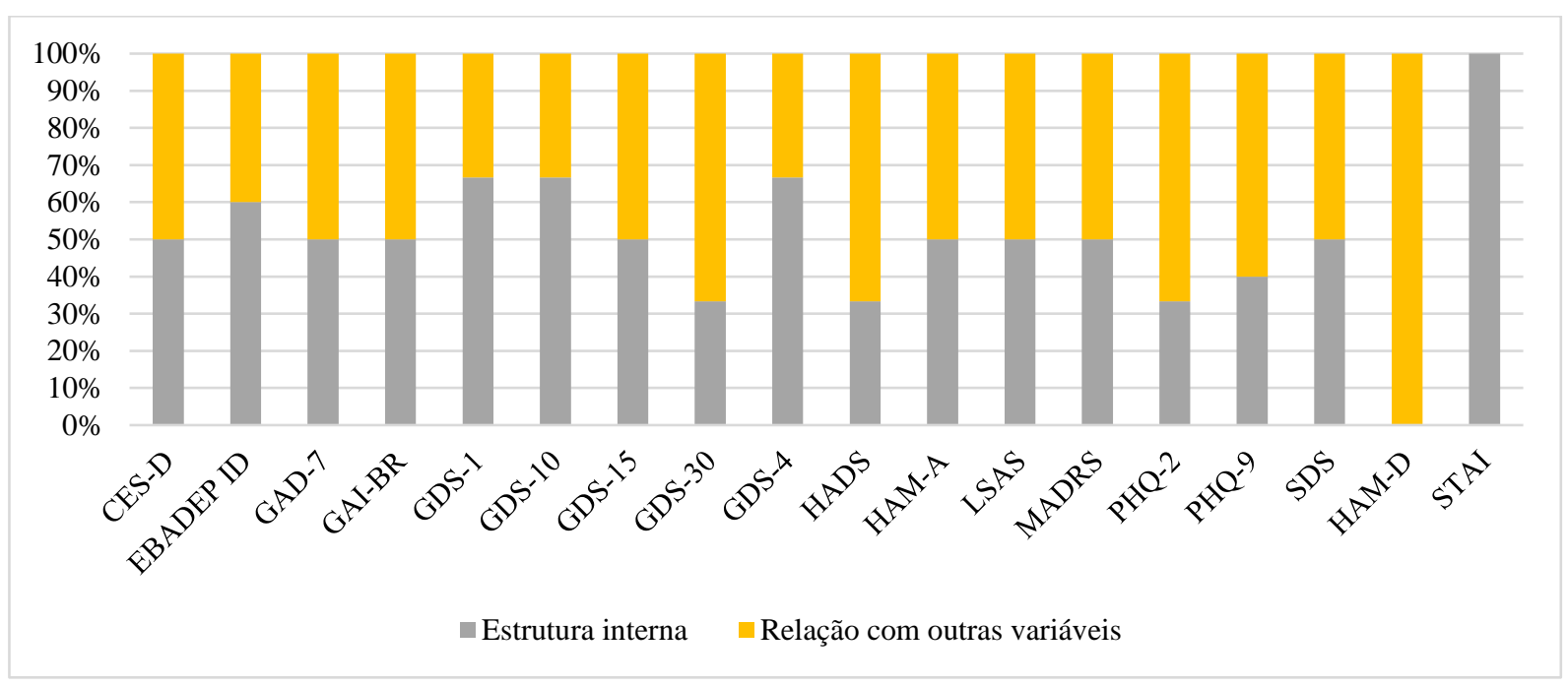

Fonte: Aline Cristina da Silva, 2021

Os demais instrumentos, com um ou dois tipos de evidências de validade, em sua maior parte se dedicaram a análises de estrutura interna ou relação com outras variáveis. Apesar da menor variabilidade de fontes de evidências, este grupo contempla os instrumentos com maior quantidade de estudos, o GDS-15 (seis estudos), seguido pelo GDS-30 (quatro estudos). Nenhum estudo se propôs a analisar evidências de validade relacionada a consequência da testagem. É reconhecida a importância de uma visão ampliada do contexto, além do teste em si, sobre o uso dos escores do teste para legitimar decisões, entretanto, ainda há certa discussão na literatura sobre esta fonte de evidência (CIZEK; BOWEN; CHURCH, 2010).

\subsection{CONCLUSÕES}

Os instrumentos de medida para avaliação de ansiedade, depressão e estresse desenvolvidos exclusivamente para idosos brasileiros ainda são raros, visto que a maioria são traduções/adaptações de outros países. Em relação as amostras, os estudos psicométricos para reunir evidências de validade para a população idosa brasileira, ainda carece de estudos exclusivos, com amplitude de faixas etárias, abordando idosos mais velhos. Quanto aos instrumentos, é importante se atentar as diferentes versões encontradas na literatura sobre o mesmo instrumento, ora de versões mais curtas, com redução de itens, ora por utilização de apenas uma subescala do instrumento que tem duas ou mais. Compreender as características dos instrumentos pode auxiliar fortemente na tomada de decisão para utilização com a população-alvo. Entretanto, para que isso seja possível, é necessária atenção do pesquisador do 
instrumento, para uma descrição adequada dos dados coletados e organização de maneira sistematizada, garantindo a compreensão dos passos executados e resultados alcançados.

A análise da qualidade metodológica, revelou pontos de atenção principalmente quanto a definição do construto a ser mensurado, bem como sua origem. A descrição operacional da variável latente e a clareza do seu conceito teórico/metodológico é ponto de partida para o desenvolvimento ou qualquer outro estudo subsequente em busca de evidências de validade do instrumento. Em relação as análises pormenorizadas dos procedimentos em buscas das evidências de validade, foi observada a falta de concordância terminológica ainda utilizada para os "tipos de validade" (conteúdo, critério e construto). O que se pode perceber também, é o descompasso entre as recomendações e o que se é praticado, sobre o conceito de evidências de validade, recomendados a partir de 1999 pelo Standards (AERA; APA; NCME, 1999) e consolidados pelas mesmas instituições no Standards de 2014. Nesse cenário, assurge em destaque a utilização em massa do uso de alfa de Cronbach para análise de consistência interna, por vezes referida como confiabilidade, mesmo que a psicometria contemporânea aponte inúmeras limitações sobre sua utilização (SIJTSMA, 2009; TRIZANO-HERMOSILLA; ALVARADO, 2016; PANAYIDES, 2013), e tem sido ignoradas na prática, mesmo com esses problemas sendo reportados há tempos, como apontam os estudos de Cortina (1993) e Schmitt (1996). A sua aplicação como consistência interna é ainda mais delicada, como aponta Tang, Cui e Babenko (2014), pois não há uma definição clara do que é consistência interna, havendo múltiplas e controversas definições. No artigo em questão, os autores destacam que nenhum dos seis índices utilizados para aferir a consistência interna teve a capacidade de avaliar a consistência interna, entre os índices, estava o alfa de Cronbach.

Os procedimentos para reunir evidências de validade a partir da estrutura interna e relação com outras variáveis, parecem ser os mais democráticos, ou pelo menos, os que mais despertam o interesse dos pesquisadores quando se fala em processo de validação. Obteve destaque os estudos de análises fatoriais/similares e análises de curva ROC. Nenhum estudo se propôs a analisar evidências de validade relacionada a consequência da testagem. Portanto, acredita-se que ainda existem passos largos a serem dados em relação a psicometria em áreas/campos da saúde, social, entre outras que se propõem a navegar nos mares de ciências transversais como a psicometria. O uso de instrumentos tem impacto direto e importante para a população idosa, aqui em destaque, mas não somente. Desprezar a literatura contemporânea e continuar utilizando desenhos de pesquisa baseado em modelos ultrapassados é irresponsável. 
Não se trata de descartar o que já foi alcançado, mas sim, questionar e aprimorar o conhecimento disponível.

\section{REFERÊNCIAS}

American Educational Research Association (AERA), American Psychological Association (APA), \& National Council on Measurement in Education (NCME). Standards for educational and psychological testing. Washington, DC: Author, 1954.

American Educational Research Association (AERA), American Psychological Association (APA), \& National Council on Measurement in Education (NCME). Standards for educational and psychological testing. Washington, DC: Author, 1965.

American Educational Research Association (AERA), American Psychological Association (APA), \& National Council on Measurement in Education (NCME). Standards for educational and psychological testing. Washington, DC: Author, 1985.

American Educational Research Association (AERA), American Psychological Association (APA), \& National Council on Measurement in Education (NCME). Standards for educational and psychological testing. Washington, DC: Author, 1999.

American Educational Research Association (AERA), American Psychological Association (APA), \& National Council on Measurement in Education (NCME). Standards for educational and psychological testing. Washington, DC: Author, 2014.

ANDRADE, J. M.; VALENTINI, F. Diretrizes para a Construção de Testes Psicológicos: a Resolução CFP n ${ }^{\circ}$ 009/2018 em Destaque. Psicol. cienc. prof., Brasília, v. 38, n. spe, p. 28-39, 2018. DOI: https://doi.org/10.1590/1982-3703000208890. Disponível em: http://www.scielo.br/scielo.php?script=sci_arttext\&pid=S1414-98932018000400028. Acesso em: 29 jan. 2021.

CATTELL, R. B. The scientific use of fator analysis. New York: Plenum, 1978.

CIZEK, G. J., BOWEN, D., CHURCH, K. Sources of Validity Evidence for Educational and Psychological Tests: A Follow-Up Study. Educational and Psychological Measurement., v. 70, n. 5, p. 732-743, 2010. DOI:10.1177/0013164410379323. Disponível em: https://journals.sagepub.com/doi/abs/10.1177/0013164410379323. Acesso em: 29 jan. 2021.

CORRÊA, C. A. et al. Níveis de estresse, ansiedade, depressão e fatores associados durante a pandemia de COVID-19 em praticantes de Yoga. RBAFS, v. 25, 2020. DOI: https://doi.org/10.12820/rbafs.25e0118. Disponível em: https://rbafs.org.br/RBAFS/article/view/14288. Acesso em: 29 jan. 2021.

CORTINA, Jose M. What is coefficient alpha? An examination of theory and applications. Journal of applied psychology, v. 78, n. 1, p. 98-104, 1993. DOI: https://doi.org/10.1037/0021-9010.78.1.98. Acesso em: https://psycnet.apa.org/record/199319965-001. Acesso em: 29 jan. 2021. 
EUGENIO, G. C. Métodos de seleção de pontos de corte em Análise de vivência. 2017. Dissertação (Mestrado em Ciências) - Instituto de Matemática e Estatística, Universidade de São Paulo, São Paulo, 2017.

EVERITT, B. S. Multivariate analysis: The need for data, and other problems. Br. J. Psychiatry, v, 126, n. 1, p 237-240, 1975. DOI: 10.1192/bjp.126.3.237. Disponível em: https://PubMed.ncbi.nlm.nih.gov/1125504/. Acesso em: 29 jan. 2021.

FERRETTI-REBUSTINI, R. E. L. Síndrome da Fragilidade em Idosos com Doença Cardiovascular: Um Olhar Sobre as Evidências de Validade dos Instrumentos de Medida. 2018. Tese (Livre Docência) - Escola de Enfermagem da Universidade de São Paulo, 2018.

GORSUCH, R. L. Factor analysis (2nd ed.). Hillsdale, NJ: Erlbaum, 1983.

HAIR, J. F. et al. Análise multivariada de dados. SANT'ANNA, A. S.; CHAVES. NETO A. (Trad.). Porto Alegre: Bookman, 2005.

HIGGINS, J.P.T.; THOMAS, J.; CHANDLER, J.; CUMPSTON, M.; LI, T.; PAGE, M.J.; WELCH, V.A. (editors). Cochrane Handbook for Systematic Reviews of Interventions version 6.0 (updated July 2019). Cochrane, 2019. Disponível em: https://training.cochrane.org/handbook. Acesso em: 25 fev. 2020

LOWSKY, D. J. et al. Heterogeneity in healthy aging. J. Gerontol. A Biol. Sci. Med. Sci. v. 69, n. 6, p. 640-649, 2014. DOI:10.1093/gerona/glt162. Disponível em: https://PubMed.ncbi.nlm.nih.gov/24249734/. Acesso em: 29 jan. 2021.

MAXIMIANO-BARRETO, M. A.; FERMOSELI, A. F. O. Prevalência de ansiedade e depressão em idosos de baixa escolaridade em Maceió/Al. Psic., Saúde \& Doenças, Lisboa, v. 18, n. 3, p. 801-813, 2017. DOI: http://dx.doi.org/10.15309/17psd180314. Disponível em: http://www.scielo.mec.pt/scielo.php?script=sci_arttext\&pid=S1645-00862017000300014.

Acesso em: 29 jan. 2021.

MENEZES-SILVA, R. et al. Epidemiological survey in elderly people (II): oral health, anxiety, depression, stress, and drug utilization. Scientia Médica, v. 26, n. 1, p. ID21980, 2016. DOI: https://doi.org/10.15448/1980-6108.2016.1.21980. Disponível em: https://revistaseletronicas.pucrs.br/ojs/index.php/scientiamedica/article/view/21980. Acesso em: 29 jan. 2021.

MESSICK, S. Significado e valores na validação de testes: a ciência e a ética da avaliação. Pesquisador educacional, v. 18, p. 5-11, 1989. DOI: 10.3102 / 0013189X018002005. Disponível em: https://journals.sagepub.com/doi/abs/10.3102/0013189X018002005. Acesso em: 29 jan. 2021.

MOHER, D. et al. The PRISMA Group. Preferred Reporting Items for Systematic Reviews and Meta-Analyses: The PRISMA Statement. PLoS Medicine, v. 6, n. 7, 2009. DOI: https://doi.org/10.1371/journal.pmed.1000097. Disponível em https://journals.plos.org/plosmedicine/article?id=10.1371/journal.pmed.1000097. Acesso em: 23 fev. 2020. 
MOKKINK, L. B. et al. COSMIN Risk of Bias checklist for systematic reviews of PatientReported Outcome Measures. Qual. Life Res., v.27, n. 5, p. 1171-1179, 2018. DOI: 10.1007/s11136-017-1765-4. Disponível em: https://PubMed.ncbi.nlm.nih.gov/29260445/. Acesso em: 29 jan. 2021.

NORONHA, A. P. P.; VENDRAMINI, C. M. M. Parâmetros psicométricos: estudo comparativo entre testes de inteligência e de personalidade. Psicol. Reflex. Crit., Porto Alegre, v. 16, n. 1, p. 177-182, 2003. DOI: http://dx.doi.org/10.1590/S0102-79722003000100018. Disponível em: http://www.scielo.br/scielo.php?script=sci_arttext\&pid=S010279722003000100018. Acesso em: 29 jan. 2021.

OLIVEIRA, D. V. et al. Investigação dos fatores psicológicos e emocionais de idosos frequentadores de clubes de dança de salão. Rev. bras. geriatr. gerontol., Rio de Janeiro, v. 20, n. 6, p. 797-804, 2017. DOI: http://dx.doi.org/10.1590/1981-22562017020.170089. Disponível em: http://www.scielo.br/scielo.php?script=sci_arttext\&pid=S180998232017000600797. Acesso em: 29 jan. 2021.

PANAYIDES, P. Coefficient alpha: Interpret with caution. Europe's Journal of Psychology, v. 9, n. 4, p. 687-696, 2013. DOI: https://doi.org/10.5964/ejop.v9i4.653. Disponível em: https://ejop.psychopen.eu/index.php/ejop/article/view/653. Acesso em: 29 jan. 2021.

PRIMI, R.; MUNIZ, M.; NUNES, C. H. S. S. Definições contemporâneas de validade de testes psicológicos. In: SHUTZ, C. S. Avanços e polêmicas em avaliação psicológica. Itatiba: Casa do Psicólogo, 2009.

RODRIGUES, C. F. S.; LIMA, F. J. C.; BARBOSA, F. T. Importância do uso adequado da estatística básica nas pesquisas clínicas. Rev. Bras. Anestesiol., Campinas, v. 67, n. 6, p. 619625, 2017. DOI: https://doi.org/10.1016/j.bjane.2017.01.011. Disponível em: http://www.scielo.br/scielo.php?script=sci_arttext\&pid=S0034-70942017000600619. Acesso em: 24 jan. 2021.

SIJTSMA, K. On the use, the misuse, and the very limited usefulness of Cronbach's alpha. Psychometrika, v. 74, n. 1, p. 107, 2009. DOI: 10.1007/s11336-008-9101-0. Disponível em: https://www.ncbi.nlm.nih.gov/pmc/articles/PMC2792363/. Acesso em: 29 jan. 2021.

TALARICO, J. N. S. et al. Sintomas de estresse e estratégias de coping em idosos saudáveis. Rev. esc. enferm. USP, São Paulo, v. 43, n. 4, p. 803-809, 2009. DOI: https://doi.org/10.1590/S0080-62342009000400010. Disponível em: http://www.scielo.br/scielo.php?script=sci_arttext\&pid=S0080-62342009000400010. Acesso em: 29 jan. 2021.

TANAKA, O. Y.; TAMAKI, E. M. O papel da avaliação para a tomada de decisão na gestão de serviços de saúde. Ciênc. saúde coletiva, Rio de Janeiro, v. 17, n. 4, p. 821-828, 2012. DOI: http://dx.doi.org/10.1590/S1413-81232012000400002. Disponível em: http://www.scielo.br/scielo.php?script=sci_arttext\&pid=S1413-81232012000400002. Acesso em: 29 jan. 2021.

TANG, W.; CUI, Y.; BABENKO, O. Internal consistency: Do we really know what it is and how to assess it. Journal of Psychology and Behavioral Science, v. 2, n. 2, p. 205-220, 2014. 
ISSN: 2374-2380 (Print) 2374-2399 (Online). Disponível em: http://jpbsnet.com/journals/jpbs/Vol_2_No_2_June_2014/13.pdf. Acesso em: 29 jan. 2021.

TRIZANO-HERMOSILLA, I.; ALVARADO, J. M. Best alternatives to Cronbach's alpha reliability in realistic conditions: congeneric and asymmetrical measurements. Front. Psychol., v. 7, p. 769, 2016. DOI: 10.3389/fpsyg.2016.00769. Disponível em: https://www.ncbi.nlm.nih.gov/pmc/articles/PMC4880791/. Acesso em: 29 jan. 2021.

\section{*Referências dos artigos incluídos na síntese desta revisão sistemática}

ALMEIDA, O. P.; ALMEIDA, S. A. Confiabilidade da versão brasileira da Escala de Depressão em Geriatria (GDS) versão reduzida. Arq. Neuro-Psiquiatr., v. 57, n. 2B, p. 421 426, 1999. DOI: http://dx.doi.org/10.1590/S0004-282X1999000300013. Disponível em: http://www.scielo.br/scielo.php?script=sci_arttext\&pid=S0004-

282X1999000300013\&lng=en\&nrm=iso>. Acesso em: 01 fev. 2021.

ALMEIDA, C. B. L. et al. Pain-induced depression in the elderly: Validation of psychometric properties of the Brazilian version of the "Geriatric Emotional Assessment of Pain" - GEAP-b. Rev. Assoc. Med. Bras., v. 63, n. 9, p. 741-746, 2017. DOI: https://doi.org/10.1590/18069282.63.09.741. Disponível

http://www.scielo.br/scielo.php?script=sci_arttext\&pid=S0104-

em: $42302017000900741 \& \operatorname{lng}=$ en\&nrm=iso. Acesso em: 01 fev. 2021.

ALVARENGA, M. R. M; OLIVEIRA, M. A. C.; FACCENDA, O. Sintomas depressivos em idosos: análise dos itens da Escala de Depressão Geriátrica. Acta Paulista de Enfermagem, v. 25, n. 4, p. 497-503, 2012. https://doi.org/10.1590/S0103-210020120004000035. Disponível em: https://www.scielo.br/pdf/ape/v25n4/03.pdf. Acesso em: 01 fev. 2021.

BAPTISTA, M. N.; SANTOS, L. M.; FILHO, N. H. Análise bifator da Escala Baptista de Depressão - Versão Idosos (EBADEP-ID). Psico, v. 50, n. 2, p. e29948, 2019. DOI: https://doi.org/10.15448/1980-8623.2019.2.29948. Disponível em: https://revistaseletronicas.pucrs.br/ojs/index.php/revistapsico/article/view/29948. Acesso em: $01 \mathrm{fev} .2021$.

BAPTISTA, M. N.; CUNHA, F. A.; MARQUES, M. A. N. Evidências de estrutura interna da Escala Baptista de Depressão: Versão Idoso (EBADEP-ID). Psicol. pesq., v. 13, n. 1, p. 76-85, 2019. DOI: http://dx.doi.org/10.24879/2018001200300478. Disponível em <http://pepsic.bvsalud.org/scielo.php?script=sci_arttext\&pid=S1982$12472019000100008 \& \operatorname{lng}=$ pt\&nrm=iso>. acessos em 01 fev. 2021.

BARCA M. L., et al. Confirmatory factor analysis of the Cornell scale for depression in dementia among patient with dementia of various degrees. J Affect Disord., v. 1, n. 188, p. 173-178, 2015. DOI:10.1016/j.jad.2015.08.062. Disponível em: https://PubMed.ncbi.nlm.nih.gov/26363614/. Acesso em: 01 fev. 2021.

BATISTONI, S. S. T.; NERI, A. L.; CUPERTINO, A. P. F. B. Validade da escala de depressão do Center for Epidemiological Studies entre idosos brasileiros. Rev. Saúde Pública, v. 41, n. 4, p. 598-605, 2007. DOI: https://doi.org/10.1590/S0034-89102007000400014. Disponível em: http://www.scielo.br/scielo.php?script=sci_arttext\&pid=S003489102007000400014\&lng=en\&nrm=iso>. Acesso em: 01 fev. 2021. 
BENZONI, Paulo Eduardo. Construction and validation of the Adult Stressors Inventory (ASI). Trends Psychiatry Psychother., v. 41, n. 4, p. 375-386, 2019. DOI: http://dx.doi.org/10.1590/2237-6089-2018-0079. Disponível em: http://www.scielo.br/scielo.php?script=sci_arttext\&pid=S2237-

60892019000400375\&lng=en\&nrm=iso. Acesso em: 01 fev. 2021.

CARTHERY-GOULART, M. T. et al. Versão brasileira da Escala Cornell de depressão em demência (Cornell depression scale in dementia). Arq. Neuro-Psiquiatr. [online]. v. 65, n. 3b, p.912-915, 2007. DOI: https://doi.org/10.1590/S0004-282X2007000500037. Disponível em: https://www.scielo.br/scielo.php?pid=S0004-

282X2007000500037\&script=sci_abstract\&tlng=pt. Acesso em: 01 fev. 2021.

CASTELO, M. S. et al. Validity of the Brazilian version of the Geriatric Depression Scale (GDS) among primary care patients. International psychogeriatrics, v. 22, n. 1, p. 109-113, 2010. DOI: https://doi.org/10.1017/S1041610209991219. Disponível em: https://search.proquest.com/openview/07ada3c21 ecffce6758ba4865708141e/1?pqorigsite $=$ gscholar \&cbl=31252. Acesso em: 01 fev. 2021.

CHACHAMOVIC, E.; FLECK, M. P.; POWER, M. Is Geriatric Depression Scale-15 a suitable instrument for measuring depression in Brazil? Results of a Rasch analysis. Psychology, Health \& Medicine, v. 15, n. 5, p. 596-606, 2010. DOI: https://doi.org/10.1080/13548506.2010.487108. Disponível em: https://europepmc.org/article/med/20835969. Acesso em: 01 fev. 2021.

CHAGAS, M. H. et al. Validity of a Brazilian version of the Zung self-rating depression scale for screening of depression in patients with Parkinson's disease. Parkinsonism Relat Disord., v. 16, n. 1, p. 42-45, 2010. DOI: 10.1016/j.parkreldis.2009.07.010. Disponível em: https://PubMed.ncbi.nlm.nih.gov/19660977/. Acesso em: 01 fev. 2021.

CHAGAS M. H., et al. Validity of the PHQ-2 for the screening of major depression in Parkinson's disease: two questions and one important answer. Aging Ment Health., v. 15, n. 7 , p. 838-843, 2011. DOI:10.1080/13607863.2011.569482. Disponível em: https://PubMed.ncbi.nlm.nih.gov/21562987/. Acesso em: 01 fev. 2021.

COSTA, E. et al. Is the GDS-30 better than the GHQ-12 for screening depression in elderly people in the community? The Bambui Health Aging Study (BHAS). Int Psychogeriatr., v. 18, n. 3, p. 493-503, 2006. DOI:10.1017/S1041610205002954. Disponível em: https://PubMed.ncbi.nlm.nih.gov/16478572/. Acesso em: 01 fev. 2021.

COSTA, E. M. D. et al. Agreement Between the Geriatric Depression Scale and the General Health Questionnaire in a Population-Based Elderly Cohort. Clinical Gerontologist, v. 26, n. 3-4, p. 69-82. DOI: 10.1300/J018v26n03_06. Disponível em. Acesso em: 01 fev. 2021.

COSTA, M. V. et al. Accuracy of three depression screening scales to diagnose major depressive episodes in older adults without neurocognitive disorders. Rev. Bras. Psiquiatr., v. 38, n. 2, p. 154-156, 2016. DOI: https://doi.org/10.1590/1516-4446-2015-1818. Disponível em: $\quad$ http://www.scielo.br/scielo.php?script=sci_arttext\&pid=S151644462016000200154\&lng=en\&nrm=iso>. Acesso em: 01 fev. 2021. 
COUTINHO, F. L.; HAMDAN, A. C.; BAPTISTA, M. N. Escala Baptista de Depressão para Idosos - EBADEP-ID: evidências de validade. Perspectivas en Psicología: Revista de Psicología y Ciencias Afines, v. 13, n. 2, 2016. Disponível em: http://www.redalyc.org/articulo.oa?id=483551472007. Acesso em: 01 fev. 2021.

DIAS, F. L. C. et al. Accuracy of the 15-item Geriatric Depression Scale (GDS-15) in a community-dwelling oldest-old sample: the Pietà Study. Trends Psychiatry Psychother., v. 39, n. 4, p. 276-279, 2017. DOI: http://dx.doi.org/10.1590/2237-6089-2017-0046. Disponível em: $\quad$ http://www.scielo.br/scielo.php?script=sci_arttext\&pid=S223760892017000400276\&lng=en\&nrm=iso>. Acesso em: 01 fev. 2021.

FARO, A. Análise Fatorial Confirmatória e Normatização da Hospital Anxiety and Depression Scale (HADS). Psic.: Teor. e Pesq. [online], vol.31, n.3, p.349-353, 2015. DOI: https://doi.org/10.1590/0102-37722015032072349353. Disponível em: https://www.scielo.br/scielo.php?pid=S0102-

37722015000300349\&script=sci_abstract\&tlng=pt. Acesso em: 01 fev. 2021.

FERRETTI-REBUSTINI, R.E.L. Síndrome da fragilidade em idosos com doença cardiovascular: um olhar sobre as evidências de validade dos instrumentos de medida. 2018. 160 f. Tese (Livre-Docência em Enfermagem Gerontológica) - Escola de Enfermagem da Universidade de São Paulo, 2018.

GIAVONI, A. et al. Elaboration and validation of the Depression Scale for the Elderly. Cadernos de Saúde Pública., v. 24, n. 5, p. 975-982, 2008. DOI: 10.1590/s0102311x2008000500004. Disponível em: https://europepmc.org/article/med/18461226. Acesso em: 01 fev. 2021.

KAIPPER, M. B. et al. Evaluation of the structure of Brazilian State-Trait Anxiety Inventory using a Rasch psychometric approach. J Psychosom Res., v. 68, n. 3, p. 223-233, 2010. DOI:10.1016/j.jpsychores.2009.09.013. Disponivel em: https://PubMed.ncbi.nlm.nih.gov/20159207/. Acesso em: 01 fev. 2021.

KUMMER, A.; CARDOSO, F.; TEIXEIRA, A. L. Frequency of social phobia and psychometric properties of the Liebowitz social anxiety scale in Parkinson's disease. Mov Disord., v. 23, n. 12, p. 1739-1743, 2008. DOI: 10.1002/mds.22221. Disponível em: https://PubMed.ncbi.nlm.nih.gov/18661550/. Acesso em: 01 fev. 2021.

KUMMER, A.; CARDOSO, F.; TEIXEIRA, A. L. Generalized anxiety disorder and the Hamilton Anxiety Rating Scale in Parkinson's disease. Arq. Neuro-Psiquiatr., v. 68, n. 4, p. 495-501, 2010. DOI: https://doi.org/10.1590/S0004-282X2010000400005. Disponível em: http://www.scielo.br/scielo.php?script=sci_arttext\&pid=S0004-

282X2010000400005\&lng=en\&nrm=iso>. Acesso em: 01 fev. 2021.

LUFT, C. Di B. et al. Versão brasileira da Escala de Estresse Percebido: tradução e validação para idosos. Rev. Saúde Pública, v. 41, n. 4, p. 606-615, 2007. DOI: https://doi.org/10.1590/S0034-89102007000400015. Disponível em: http://www.scielo.br/scielo.php?script=sci_arttext\&pid=S003489102007000400015\&lng=en\&nrm=iso. Acesso em: 01 fev. 2021. 
MASSENA P. N. et al. Validation of the Brazilian Portuguese Version of Geriatric Anxiety Inventory--GAI-BR. Int Psychogeriatr., v. 27, n. 7, p. 1113-1119, 2015. DOI:10.1017/S1041610214001021. Disponível https://PubMed.ncbi.nlm.nih.gov/24946782/. Acesso em: 01 fev. 2021.

MONTEIRO, R. P. et al. Psychometric Evidence of the 7-Item Generalized Anxiety Disorder Questionnaire in Brazil. International Journal of Mental Health and Addiction, 2020. Disponível em: https://link.springer.com/article/10.1007/s11469-020-00423-9. Acesso em: 01 fev. 2021.

OSÓRIO, F. L. et al. Are two items sufficient to screen for depression within the hospital context?. Int J Psychiatry Med., v. 44, n. 2, p. 141-148, 2012. DOI: 10.2190/PM.44.2.e. Disponível em: https://PubMed.ncbi.nlm.nih.gov/23413661/. Acesso em: 01 fev. 2021.

PARADELA, E. M. P.; LOURENCO, R. A.; VERAS, R. P. Validation of geriatric depression scale in a general outpatient clinic. Rev. Saúde Pública, v. 39, n. 6, p. 918-923, 2005. DOI: https://doi.org/10.1590/S0034-89102005000600008. Disponível em: http://www.scielo.br/scielo.php?script=sci_arttext\&pid=S003489102005000600008\&lng=en\&nrm=iso. Acesso em: 01 fev. 2021.

PEDROSO, V. S. P. et al. Psychopathological evaluation and use of the Hospital Anxiety and Depression Scale in a sample of Brazilian patients with post-stroke depression. Arch. Clin. Psychiatry (São Paulo), v. 43, n. 6, p. 147-150, 2016. DOI: https://doi.org/10.1590/010160830000000102.

em:

http://www.scielo.br/scielo.php?script=sci_arttext\&pid=S0101-

60832016000600147\&lng=en\&nrm=iso. Acesso em: 01 fev. 2021.

PINHO, M. X. et al. Confiabilidade e validade da escala de depressão geriátrica em idosos com doença arterial coronariana. Arq. Bras. Cardiol., v. 94, n. 5, p. 570-579, 2010. DOI: https://doi.org/10.1590/S0066-782X2010005000032. Disponível em: http://www.scielo.br/scielo.php?script=sci_arttext\&pid=S0066782X2010000500001\&lng=en\&nrm=iso. Acesso em: 01 fev. 2021.

PORTUGAL, M. da G. et al. Validation of Montgomery-Åsberg Rating Scale and Cornell Scale for Depression in Dementia in Brazilian elderly patients. Int Psychogeriatr. v. 24, n. 8, p. 1291-1298, 2012. DOI:10.1017/S1041610211002250. Disponível em: https://PubMed.ncbi.nlm.nih.gov/22185723/. Acesso em: 01 fev. 2021.

SARDÁ, J. JR. et al. Psychometric properties of the DASS-Depression scale among a Brazilian population with chronic pain. J Psychosom Res. v. 64, n. 1, p. 25-31, 2008. DOI:10.1016/j.jpsychores.2007.05.015. Disponível em: https://PubMed.ncbi.nlm.nih.gov/18157996/. Acesso em: 01 fev. 2021.

SILBERMAN, C. D. et al. Recognizing depression in patients with Parkinson's disease: accuracy and specificity of two depression rating scale. Arq. Neuro-Psiquiatr., v. 64, n. 2b, p. 407-411, 2006. DOI: http://dx.doi.org/10.1590/S0004-282X2006000300011. Disponível em: http://www.scielo.br/scielo.php?script=sci_arttext\&pid=S0004-

282X2006000300011\&lng=en\&nrm=iso. Acesso em: 01 fev. 2021. 
TERRA, Mauro B et al. Internal consistency and factor structure of the Portuguese version of the Liebowitz Social Anxiety Scale among alcoholic patients. Rev. Bras. Psiquiatr., v. 28, n. 4, p. 265-269, 2006. DOI: https://doi.org/10.1590/S1516-44462006005000008. Disponível em: $\quad$ http://www.scielo.br/scielo.php?script=sci_arttext\&pid=S1516$44462006000400004 \& \operatorname{lng}=\mathrm{en} \& n r m=\mathrm{iso}>$. Acesso em: 01 fev.2021.

VARGAS, H. O.; MATSUO, T.; BLAY, S. L. CrossRef citations to date Altmetric Original Articles Validity of the Geriatric Depression Scale for Patients Seen at General Outpatient Clinics. Clinical Gerontologist., v. 30, n. 4, p. 65-78, 2007. DOI: https://doi.org/10.1300/J018v30n04_05.

Disponível

https://www.tandfonline.com/doi/abs/10.1300/J018v30n04_05. Acesso em: 01 fev. 2021.

VIGNOLA, R. C. B.; TUCCI, A. M. Adaptation and validation of the depression, anxiety and stress scale (DASS) to Brazilian Portuguese. Journal of Affective Disorders. Elsevier, v. 155, p. 104-109, 2014. DOI: http://dx.doi.org/10.1016/j.jad.2013.10.031. Disponível em: http://repositorio.unifesp.br/handle/11600/37373. Acesso em: 01 fev. 2021.

\section{CONSIDERAÇÕES FINAIS}

Mediante à revisão de escopo apresentada nesta dissertação, observou-se a variedade de instrumentos de medida disponíveis para avaliação em idosos brasileiros, entretanto, a quantidade não garante a qualidade que estes instrumentos oferecem. Este ponto foi explorado sutilmente no primeiro artigo e é confirmado no segundo artigo, sobre a revisão das evidências de validade dos instrumentos para ansiedade, depressão e estresse, onde uma análise mais detalhada de cada estudo foi realizada e apresentou dados curiosos sobre as evidências recuperadas. As ciências da saúde e sociais, se utilizam fortemente destes instrumentos, isoladamente ou como parte integrante de protocolos de avaliação, o que destaca a importância da compreensão além da finalidade amplamente disseminada do instrumento, mas sobre sua constituição e sobre suas evidências de validade, ou seja, o quanto é recomendável para uso. Vale ressaltar que apesar do foco nos instrumentos de medida, um processo de avaliação, exige preparo, qualificação e olhar ampliado sobre o sujeito, garantido que sua individualidade não seja diminuída frente a classificações e rótulos descabidamente.

Os estudos analisados, 237 na scoping review e 37 na revisão sistemática, possibilitaram compreender como a comunidade científica vem realizando os procedimentos que chancelam as fontes de evidências de validade. Desde o relato baseado em terminologias e conceitos desatualizados até as tomadas de decisões frente as etapas procedimentais, como são executadas, interpretadas e dispostas como resultados. Ficou evidenciado que alguns tipos de fonte de evidência, como as baseadas na consequência da testagem, ainda são pouquíssimo exploradas, o que reflete até a falta de consenso no tema, mas também, que há pouca extensão 
de todas as evidências sobre os instrumentos de ansiedade, depressão e estresse, quando se fala da população idosa brasileira. Dos 25 instrumentos analisados, $48 \%$ tinha apenas um estudo, que pode ter contemplado mais uma fonte de evidência, mas não de forma completa e adequada como orientado na literatura. Este cenário pode ser diferente levando em consideração outras populações e localidades, entretanto, é importante saber que as evidências de validade, estão ligadas ao escore do teste, ou seja, a população e contexto em questão, por isso não se pode generalizar um instrumento amplamente conhecido e utilizado como validado, sem antes conhecer e checar suas evidências para a população idosa brasileira, neste caso específico.

Além disso, é sabido que a psicometria, assim como cada área tem suas teorias, vocabulário, terminologias e métodos, se tornando essencial aos estudiosos que se debrucem e se atualizam constantemente sobre as técnicas empregadas mais adequadas, bem como suas renovações periódicas. A comunicação entre áreas científicas é essencial quando se fala de instrumento de medida e por isso, é necessário que o pesquisador faça uma descrição adequada do estudo metodológico psicométrico realizado, facilitando a compreensão do objetivo em relação aos resultados alcançados e principalmente quanto aos procedimentos adotados, sequencialmente, de forma clara e objetiva, sendo passível de reprodução e diálogo. Apesar do rigor adotado e criteriosa análise realizada, este estudo aponta limitações quanto as suas buscas e análises, que podem não ter contemplado toda a temática e escolhido estratégias que acabaram eliminando outras visões, entretanto, acredita-se que este pode contribuir grandemente para a Gerontologia especificamente, que por ser um campo novo de atuação, necessita mergulhar profundamente nos processos de avaliação de sua população alvo, garantindo uma atuação responsável profissionalmente, mas também com todas as outras áreas/campos interessadas pela temática.

\section{REFERÊNCIAS}

ALEXANDRE, N. M. C.; COLUCI, M. Z. O. Validade de conteúdo nos processos de construção e adaptação de instrumentos de medidas. Ciênc. saúde coletiva, v. 16, n. 7, p. 3061 3068, 2011. DOI: https://doi.org/10.1590/S1413-81232011000800006. Disponível em: http://www.scielo.br/scielo.php?script=sci_arttext\&pid=S1413-

$81232011000800006 \& \operatorname{lng}=e n \& n r m=$ iso. Acesso em: 01 fev. 2021.

ALKEMA, G. E.; ALLEY, D. E. Gerontology's future: An integrative model for disciplinary advancement. The Gerontologist, v. 46, n. 5, p. 574-582, 2006. DOI: 10.1093/geront/46.5.574. Disponível em: https://www.ncbi.nlm.nih.gov/PubMed/17050748. Acesso em: 09 fev. 2020. 
ALVES, M. C. C. Envelhecimento, rede social e funcionalidade na vida diária: um estudo nos centros de dia e universidade sénior de Guimarães. 2017. f. Dissertação (Mestrado em Gerontologia Social) - Instituto Politécnico de Viana Castelo, Portugal, 2017.

American Educational Research Association (AERA), American Psychological Association (APA), \& National Council on Measurement in Education (NCME). Standards for educational and psychological testing. Washington, DC: Author, 1954.

American Educational Research Association (AERA), American Psychological Association (APA), \& National Council on Measurement in Education (NCME). Standards for educational and psychological testing. Washington, DC: Author, 1985.

American Educational Research Association (AERA), American Psychological Association (APA), \& National Council on Measurement in Education (NCME). Standards for educational and psychological testing. Washington, DC: Author, 1999.

American Educational Research Association (AERA), American Psychological Association (APA), \& National Council on Measurement in Education (NCME). Standards for educational and psychological testing. Washington, DC: Author, 2014.

ANDRADE, J. M.; VALENTINI, F. Diretrizes para a Construção de Testes Psicológicos. Psicologia: Ciência e Profissão, v. 38, p. 28-39, 2018. Disponível em: https://www.scielo.br/pdf/pcp/v38nspe/1982-3703-pcp-38-nspe1-0028.pdf. Acesso em: 01 fev. 2021.

ARONSON, J.; FRIED, C. B.; GOOD, C. Reduzindo os efeitos da ameaça do estereótipo em estudantes universitários afro-americanos, moldando teorias de inteligência. Journal of Experimental Social Psychology, v. 38, n. 2, p. 113-125, 2002. DOI: 10.1006/jesp.2001.1491. Disponível: $\quad$ https://uploadsssl.webflow.com/59faaf5b01b9500001e95457/5bc5522d99e1036c0d8905f2_Aronson\%2C\%2 0Fried\%20\%26\%20Good\%2C\%202002.pdf. Acesso em: 01 fev. 2021.

ARSENIJEVIC, J.; et al. Catastrophic Health Care Expenditure among Older People with Chronic Diseases in 15 European Countries. PLOS ONE, v. 11, n. 7, 2016. DOI: https://doi.org/10.1371/journal.pone.0157765. Disponível em https://journals.plos.org/plosone/article\%3Fid\%3D10.1371\%252Fjournal.pone.0157765. Acesso em: 24 fev. 2020.

ATCHLEY, R. C. Retirement and Leisure Participation: Continuity or Crisis?. The Gerontologist, v. 11, n. 1_Part_1, p. 13-17, 1971. DOI: https://doi.org/10.1093/geront/11.1_Part_1.13. Disponível em: https://academic.oup.com/gerontologist/articleabstract/11/1_Part_1/13/512910?redirectedFrom=fulltext. Acesso em: 01 fev. 2021.

BALTES, P. B. Theoretical propositions of life-span developmental psychology: On the dynamics between growth and decline. Developmental psychology, v. 23, n. 5, p. 611-626, 1987. DOI: https://doi.org/10.1037/0012-1649.23.5.611. Disponível em: https://psycnet.apa.org/record/1988-01055-001. Acesso em: 23 fev. 2020. 
BALTES, P. B.; BALTES, M. M. Psychological perspectives on successful aging: The model of selective optimization with compensation. In: BALTES, P. B.; BALTES, M. M. (Eds). Successful aging. Perspectives from behavioral sciences (pp. 1-34). Cambridge: Cambridge University Press, 1990.

BANDALOS, D.L. Measurement theory and applications for the social sciences. Guilford Press, 2018. p. 661

BIGGS, S.; LOWENSTEIN, A.; HENDRICKS, J. (eds), The Need for Theory: Critical Approaches to Social Gerontology, New York: Baywood Publishing Company, 2003.

BOLEN, K. A. Latent Variables in Psychology and the Social Sciences. Annual Review of $\begin{array}{llllll}\text { Psychology, } & \text { v. } & 53, & \text { p. } & 605-634, & \text { DOI: }\end{array}$ https://doi.org/10.1146/annurev.psych.53.100901.135239. Disponível: https://www.annualreviews.org/doi/abs/10.1146/annurev.psych.53.100901.135239?casa_toke $\mathrm{n}=\mathrm{t}$ 9PWFh_tcWUAAAAA\%3AIJNfyx2gJmZQ-

8ZB8eePhPOzrpRI6J4C70sUtW8VNo8YbX_luIRudpeDhERoMDEiKetwbYRQe6NU\&jour nalCode=psych. Acesso em: 01 fev. 2021.

BORSBOOM, D. Latent Variable Theory, Measurement: Interdisciplinary Research and Perspectives. Psychology Press, v. 6, n. 1-2, p. 25-53, 2008. DOI: 10.1080/15366360802035497. Disponível em: http://dx.doi.org/10.1080/15366360802035497. Acesso em: 01 fev. 2021.

BRANDSTÄDTER, J.; RENNER, G. Tenacious goal pursuit and flexible goal adjustment: Age-related analysis of assimilative and accommodative strategies of coping. Psychology of Aging, v. 5, n. 1, p. 58-67, 1990. DOI: 10.1037/0882-7974.5.1.58. Disponível: https://www.sciencedirect.com/science/article/abs/pii/S0273229701905398?via\%3Dihub.

Acesso em: 01 fev. 2021.

CAMPOS, A. C. V. Envelhecimento no Brasil: um processo multidimensional. Enfermagem Brasil, $\quad$ v. $10, \quad$ n. $5, \quad 2011.2$ Disponível http://portalatlanticaeditora.com.br/index.php/enfermagembrasil/article/view/3871/5870.

Acesso em: 09 fev. 2020.

CAMPISI, J. Envelhecimento, senescência celular e câncer. Annual Review Physiology, v. 75, p. 685-705, 2013. DOI: 10.1146/annurev-physiol-030212-183653. Disponível em: https://www.ncbi.nlm.nih.gov/PubMed/23140366. Acesso em: 23 fev. 2020.

CAMPOS, K. B.; PÉCORA, A. R. Envelhecer adoecendo: Relatos de pacientes idosos internados no Hospital Universitário Júlio Muller, Cuiabá-MT. Estudos Interdisciplinares sobre o Envelhecimento, v. 20, n. 2, p. 625-643, 2015. Disponível: https://seer.ufrgs.br/RevEnvelhecer/article/view/40918/35462. Acesso em: 23 fev. 2020.

CARSTENSEN, L. L. Age-related changes in social activity. In CARSTENSEN, L. L.; EDELSTEIN, B. A. (Eds.), Pergamon general psychology series, v. 146, p. 222-237, 1987. Handbook of clinical gerontology Pergamon Press.

CARSTENSEN, L. L. Socioemotional selectivity theory: Social activity in lifespan context. Annual Review of Gerontology and Geriatrics, v. 11, p. 195-217, 1991. 
CHAN, E. K. H. Standards and Guidelines for Validation Practices: Development and Evaluation of Measurement Instruments. In: Validity and Validation in Social, Behavioral, and Health Sciences. Canadá: Springer, 2014. p. 9-23.

CHARLES, S. T. Strength and vulnerability integration: A model of emotional well-being across adulthood. Psychological Bulletin, v. 136, n. 6, p. 1068-1091, 2010. DOI:10.1037/a0021232. Disponível em: https://PubMed.ncbi.nlm.nih.gov/21038939/. Acesso em: 01 fev. 2021.

COLUCI, M. Z. O.; ALEXANDRE, N. M. C.; MILANI, D. Construção de instrumentos de medida na área da saúde. Ciência \& Saúde Coletiva, v. 20, n. 3, p. 925-936, 2015. DOI: https://doi.org/10.1590/1413-81232015203.04332013.

Disponível

em: https://www.scielosp.org/article/csc/2015.v20n3/925-936/. Acesso em: 26 fev. 2020.

COUTO, G; PRIMI, R. Teoria de resposta ao item (TRI): Conceitos elementares dos modelos para itens dicotômicos. Bol. psicol, v. 61, n. 134, p. 1-15, 2011. Disponível em http://pepsic.bvsalud.org/scielo.php?script=sci_arttext\&pid=S0006-

59432011000100002\&lng=pt\&nrm=iso. Acesso em: 01 fev. 2021.

CUMMING, E.; HENRY, W. Growing Old: The Process of Disengagement. Basic Books. Ageing \& Society, v. 11, n. 2, p. 217-220, 1961. DOI: https://doi.org/10.1017/S0144686X00004025. Disponível: https://www.cambridge.org/core/journals/ageing-and-society/article/abs/cumming-e-andhenry-w-growing-old-the-process-of-disengagement-basic-books-new-york-1961-reprintarno-new-york-1979-isbn-0405-118147/8902DA0CA80B97235183711DA0BFB59A. Acesso em: 01 fev. 2021.

CUNHA, C. M.; ALMEIDA NETO, O. P.; STACKFLETH, R. Principais métodos de avaliação psicométrica da validade de instrumentos de medida. Revista de Atenção à Saúde, v. 14, n. 47, p. 75-83, 2016. DOI: https://doi.org/10.13037/ras.vol14n47.3391. Disponível em: https://seer.uscs.edu.br/index.php/revista_ciencias_saude/article/view/3391. Acesso em: 23 fev. 2020.

CURADO, M. A. S.; TELES, J.; MAROCO, J. Análise de variáveis não diretamente observáveis: influência na tomada de decisão durante o processo de investigação. Rev. esc. enferm., v. 48, n. 1, p. 146-152, 2014. DOI: http://dx.doi.org/10.1590/S0080$623420140000100019 . \quad$ Disponível

http://www.scielo.br/scielo.php?script=sci_arttext\&pid=S0080-

62342014000100146\&lng=en\&nrm=iso>. Acesso em: 01 fev. 2021.

DANIELEWICZ, A. L.; d'ORSI, E.; BOING, A.F. Renda contextual e incidência de incapacidade: resultados da Coorte EpiFloripa Idoso. Revista de Saúde Pública, v. 53, n. 31, 2019. DOI: https://doi.org/10.11606/S1518-8787.2019053000659 Disponível em: https://www.scielosp.org/article/rsp/2019.v53/11/pt/. Acesso em: 24 fev. 2020.

DARDENGO, C. F. R.; MAFRA, S. C. T. Os conceitos de velhice e envelhecimento ao longo do tempo: contradição ou adaptação?. Revista de Ciências Humanas, v. 18, n. 2, 2018. Disponível em: https://periodicos.ufv.br/RCH/article/view/8923. Acesso em: 01 fev. 2021. 
DOLL, J; et al. Atividade, desengajamento, modernização: teorias sociológicas clássicas sobre o envelhecimento. Estudos Interdisciplinares sobre o Envelhecimento, v. 12, p. 7-33, 2007. Disponível: https://seer.ufrgs.br/RevEnvelhecer/article/view/4977. Acesso em: 23 fev. 2020.

DOS SANTOS, D.F.; MOREIRA, M.A.A.; CERVENY, C. Velhice - considerações sobre o envelhecimento: imagens no espelho. Nova Perspectiva Sistêmica, v. 23, n. 48, p. 80-94, 2016. Disponível em: https://www.revistanps.com.br/nps/article/view/53. Acesso em: 01 fev. 2021.

FERNANDES, E. S. DE O.; SANTOS, A. A. A. Testes de Atenção para Idosos: Relações com Idade, Escolaridade e Moradia. Psico, v. 46, n. 1, p. 110-119, 17 mar. 2015. DOI: https://doi.org/10.15448/1980-8623.2015.1.17281. Disponível em: https://revistaseletronicas.pucrs.br/ojs/index.php/revistapsico/article/view/17281. Acesso em: $01 \mathrm{fev} .2021$.

FERRARO, K. F. Imagining the Disciplinary Advancement of Gerontology: Whither the Tipping Point?. The Gerontologist, v. 46, n. 5, p. 571-573, 2006. DOI: https://doi.org/10.1093/geront/46.5.571. Disponível em: https://academic.oup.com/gerontologist/article/46/5/571/629261. Acesso em: 09 fev. 2020.

FILHO, N. H. Medida psicológica: o debate entre as perspectivas conceituais representacionista e realista. Aval. psicol., v. 13, n. 3, p. 399-408, 2014. Disponível em: http://pepsic.bvsalud.org/scielo.php?script=sci_arttext\&pid=S1677-

04712014000300012\&lng=en\&nrm=iso >. Acesso em: 01 fev. 2021.

FRANÇA, A. B. et al. Instrumentos de avaliação da qualidade de vida do idoso com Alzheimer: revisão integrativa da literatura. Revista Eletrônica de Enfermagem, v. 18, n. e1170, 2016. DOI: https://doi.org/10.5216/ree.v18.32579. Disponível: https://revistas.ufg.br/fen/article/view/32579. Acesso em: 01 fev. 2021.

FRIES, A. T.; PEREIRA, D. C. Teorias do envelhecimento humano. Revista Contexto \& Saúde, v. 11, n. 20, p. 507-514, 2011. DOI: https://doi.org/10.21527/2176-7114.2011.20.507514. Disponível em: https://revistas.unijui.edu.br/index.php/contextoesaude/article/view/1571. Acesso em: 09 fev. 2020.

FURR, R. M. Psychometrics: an introduction. 3a edição. California: London, 2018.

GAPMINDER. Data. Listo of indicators. Doubt. Geography. Documentation. Data blog. Lyfe expectancy (years). The average number of years a newborn child would live if current mortality patterns were to stay the same. Disponível em: 〈https://www.gapminder.org/data/>. Acesso em: 09 fev. 2020

HAIR, J. F. et al. Multivariate data analysis. 7th ed. London: Pearson; 2014

HAVIGHURST, R. J. Successful Aging, The Gerontologist, v. 1, n. 1, p. 8-13, 1961. DOI: https://doi.org/10.1093/geront/1.1.8. Disponível em: https://academic.oup.com/gerontologist/article-abstract/1/1/8/551930. Acesso em: 01 fev. 2021.

HECKHAUSEN, J.; SCHULZ, R. A life-span theory of control. Psychological Review, v. 102, n. 2, 284-304, 1995. DOI: https://doi.org/10.1037/0033-295X.102.2.284. Disponível em: 
https://psycnet.apa.org/doiLanding?doi=10.1037\%2F0033-295X.102.2.284. Acesso em: 01 fev. 2021.

KESZEI, A. P.; NOVAK, M.; STREINER, D. L. Introduction to health measurement scales, Journal of Psychosomatic Research, v. 68, n. 4, p. 319-323, 2010. DOI: https://doi.org/10.1016/j.jpsychores.2010.01.006. Disponível em: https://www.sciencedirect.com/science/article/pii/S0022399910000115?casa_token=eycT_zdj p5gAAAAA:3wLKSevCFIXcHjUgeyY3XEcmPeCcnu5jAM6-NnR5NU9EMZZ9CgP_rsSYxGy0o809i2aPRg-pA. Acesso em: 01 fev. 2021.

KOOIJ, D. T. A. M.; TIMS, M.; KANFER, R. Successful aging at work: The role of job crafting. In P. M. Bal, D. T. A. M. Kooij, \& D. M. Rousseau (Eds.), Aging workers and the employee-employer relationship, p. 145-161, 2015. New York, NY: Springer.

LEBRÃO, M. L. et al. Anos do Estudo SABE: antecedentes, metodologia e organização do estudo. Revista Brasileira de Epidemiologia, v. 21, n. 2, 2018. Disponível em: <http://www.scielo.br/scielo.php?script=sci_arttext\&pid=S1415-790X2018000300200>. Acesso em: 23 fev. 2020.

LIMA, P. M. R. L.; VIANA, T. C.; LAZZARINI, E. R. Velhice? Acho Ótima, Considerando a Alternativa: Reflexões sobre Velhice e Humor. Revista Mal-estar e Subjetividade, v. 9, n. 4, p. 2011. 1597-1618, Disponível em: http://pepsic.bvsalud.org/scielo.php?script=sci_arttext\&pid=S1518-61482011000400012. Acesso em: 01 fev. 2021.

LOURENÇO, R. A.; et al. Prevalência e fatores associados à fragilidade em uma amostra de idosos que vivem na comunidade da cidade de Juiz de Fora, Minas Gerais, Brasil: estudo FIBRA-JF. Ciência \& Saúde Coletiva, v. 24, n. 1, p. 35-44, 2019. DOI: https://doi.org/10.1590/1413-81232018241.29542016. Disponível em: https://www.scielosp.org/article/csc/2019.v24n1/35-44/. Acesso em: 24 fev. 2020.

LOWSKY, D. J. et al. Heterogeneity in healthy aging. J. Gerontol. A Biol. Sci. Med. Sci. v. 69, n. 6, p. 640-649, 2014. DOI:10.1093/gerona/glt162. Disponível em: https://PubMed.ncbi.nlm.nih.gov/24249734/. Acesso em; 29 jan. 2021.

MARSHALL, V. Theory informing public policy: The life course perspective as policy tool. In V. Bengtson, D. Gans, N. Putney \& M. Silversytein (Eds.), Handbook of theories of aging. New York: Springer, 2009. p. 573-593.

MARTINS, R. Envelhecimento, retrogénese do desenvolvimento motor, exercício físico e promoção da saúde. Boletim Sociedade Portuguesa de Educação Física. n. 32, p. 31-40, 2017. Disponível em: http://boletim.spef.pt/index.php/spef/article/view/220. Acesso em: 09 fev. 2020.

MARTINS, S. S. Envelhecimento ativo e o seu reflexo na qualidade de vida dos idosos. 2017. 241 f. Dissertação (Mestrado em Serviço Social) - Universidade Lusíada de Lisboa Faculdade de ciências humanas e sociais, Lisboa, 2017. Disponível em: http://repositorio.ulusiada.pt/handle/11067/3426. Acesso em: 09 fev. 2020. 
MESQUITA, S. M. O Envelhecimento e as Reformas no Sistema de Seguridade Social no Brasil Contemporâneo. Textos \& Contextos (Porto Alegre), v. 17, n. 1, p. 126 - 137, jan./jul. 2018. DOI: 10.15448/1677-9509.2017.1.27635 Disponível em: https://revistaseletronicas.pucrs.br/ojs/index.php/fass/article/view/27635/17157. Acesso em: 01 fev. 2021.

MESSICK, S. Validity of psychological assessment. Validation of inferences from person's responses and performances as scientific inquiry into score meaning. American Psychologist, v. 50, n. 9, p. 741-749, 1995.

MIRANDA, G.M.D.; MENDES, A.C.G.; SILVA, A.L.A. O envelhecimento populacional brasileiro: desafios e consequências sociais atuais e futuras. Revista brasileira de geriatria e gerontologia, v. 19, n. 3, p. 507-519, 2016. DOI: http://dx.doi.org/10.1590/180998232016019.150140. Disponível http://www.scielo.br/scielo.php?script=sci_arttext\&pid=S1809-

98232016000300507\&lng=en\&nrm=iso. Acesso em: 24 fev. 2020.

MOKKINK, L.B.; PRINSEN, C.A.C.; BOUTER, L.M.; DE VET, H.C.W.; TERWEE, C.B. The COnsensus-based Standards for the selection of health Measurement INstruments (COSMIN) and how to select an outcome measurement instrument. Brazilian Journal Physical Therapy, v. 20, n. 2, p. 105-113, 2016. Disponível em: $<$ http://www.scielo.br/scielo.php?script=sci_arttext\&pid=S1413-

$35552016000200105 \& \operatorname{lng}=$ en\&nrm=iso $>$. Acesso em: 22 fev. 2020.

MORAES, E. N. et al. Paraná. Secretaria de Estado da Saúde do Paraná. Superintendência de Atenção à Saúde. Avaliação multidimensional do idoso / SAS. Curitiba: SESA, 2018. 113 p.

MRÓZEK, S. A person with disability in strategies for solving social problems - analysis of records in perspective of Urie Bronfenbrenner's theory. Czlowiek - Niepelnosprawność Społeczeństwo. v. 49 n. 3, p. 95-114, 2020. DOI: 10.1111/jftr.120022. Disponível: http://cejsh.icm.edu.pl/cejsh/element/bwmeta1.element.ceon.journal-16e14d2b-729e-32bda8cb-7a2a7617d1dc. Acesso em: 25 jan. 2020.

MUNGAS, D. et al. Heterogeneity of cognitive trajectories in diverse older persons. Psychology and aging, v. 25, n. 3, p. 606-19, 2010. DOI:10.1037/a0019502. Disponível

em: https://www.ncbi.nlm.nih.gov/pmc/articles/PMC2943999/\#: :text=Trajectories\%20of\%20cha nge $\% 20$ in $\% 20$ cognitive, that $\% 20$ emerge $\% 20$ as\%20people\%20age. Acesso em: 01 fev. 2021.

NERI, A. L. O legado de Paul B. Baltes à Psicologia do Desenvolvimento e do Envelhecimento. Temas psicol., v. 14, n. 1, p. 17-34, 2006. Disponível em http://pepsic.bvsalud.org/scielo.php?script=sci_arttext\&pid=S1413389X2006000100005\&lng=pt\&nrm=iso $>$. Acesso em: 01 fev. 2021.

NEUGARTEN, B. Personality and the aging process. The Gerontologist, v. 12, n. 1, p. 9-15, 1972. DOI: https://doi.org/10.1093/geront/12.1_Part_1.9. Disponível em: https://psycnet.apa.org/record/1972-27008-001. Acesso em: 01 fev. 2021.

NEWTON, P.E. Macro- and Micro-Validation: Beyond the 'Five Sources' Framework for Classifying Validation Evidence and Analysis, Practical Assessment, Research and 
Evaluation, $\quad$ v. 21, n. 12, 2016. Disponível em: https://scholarworks.umass.edu/cgi/viewcontent.cgi?article=1350\&context=pare. Acesso em: $01 \mathrm{fev} .2021$.

NEWTON, P.E.; SHAW, S.D. Standards for talking and thinking about validity. Psychology Methods, v. 18, n. 3, p. 301-319, 2013. Disponível em: https://www.ncbi.nlm.nih.gov/PubMed/23834419. Acesso em: 26 fev. 2020.

OLIVEIRA, T. R.; OLIVEIRA, A. R.; NATAL, A. L. Como mensurar o que não é observável? Abordagem reflexiva e modelagem de variáveis latentes em análises de survey. $\mathbf{4 0}^{\circ}$ Encontro Anual da ANPOCS - Seminários Temáticos - ST 16 - Métodos e técnicas de pesquisa em Ciências Sociais, 2016. Disponível em: https://www.anpocs.com/index.php/papers-40encontro/st-10/st16-7/10301-como-mensurar-o-que-n-ao-e-observ-avel-abordagem-re-exivae-modelagem-de-vari-aveis-latentes-em-an-alises-de-survey/file. Acesso em: 01 fev. 2021.

PALMORE, E. Facts on aging: A short quiz. The Gerontologist, v. 17, n. 4, p. 315-320, 1977. Disponível em: http://dx.doi.org/10.1093/geront/17.4.315. Acesso em: 24 fev. 2020.

PALMORE, E. B. The Facts on aging quiz: Part two. The Gerontologist, v. 21, n. 4, p. 431 437, 1981. Disponível em: http://dx.doi.org/10.1093/geront/21.4.431. Acesso em: 24 fev. 2020.

PAPALIA, D.E., OLDS, S.W.; FELDMAN, R.D. Desenvolvimento humano. 8 ed. Porto Alegre: Artmed, 2006.

PARANÁ. Secretaria de Estado da Saúde do Paraná. Superintendência de Atenção à Saúde. Avaliação multidimensional do idoso / SAS. Curitiba, 2017. Disponível em: https://www.saude.pr.gov.br/sites/default/arquivos_restritos/files/documento/202004/avaliacaomultiddoidoso_2018_atualiz.pdf. Acesso em: 29 jan. 2021.

PASCHOAL, S. M. P. Qualidade de vida do idoso: construção de um instrumento de avaliação através do método do impacto clínico. 2004. 245 f. Tese (Doutorado em Medicina Preventiva) - Faculdade de Medicina da Universidade de São Paulo. São Paulo, 2004.

PATHATH, A. W. Theories of Aging. International Journal of Indian Psychology, v. 4, n. 3, 2017. DOI:10.25215/0403.142. Disponível em: https://ijip.in/articles/0403142/. Acesso em: 01 fev. 2021.

RAN, Z.; HOU-ZAO, C.; DE-PEI; L. The Four Layers of Aging. Cell Systems Perspective, v. 1, n. 3, p. 180-186, 2015. DOI: https://doi.org/10.1016/j.cels.2015.09.002. Disponível em: https://www.cell.com/fulltext/S2405-4712(15)00115-5\#back-bib8. Acesso em: 09 fev. 2020.

RAYCOV, T.; MARCOULIDES, G.A. Introduction to psychometric theory. 1 ed. Routledge: 2011.p. 328.

REPPOLD, C. T.; GURGEL, L. G.; HUTZ, C. S. O processo de construção de escalas psicométricas. Avaliação Psicológica, v. 13, n. 2, p. 307-310, 2014. Disponível em $<$ http://pepsic.bvsalud.org/scielo.php?script=sci_arttext\&pid=S1677-

04712014000200018\&lng=pt\&nrm=iso>. Acesso em: 26 fev. 2020.

ROBSON, S. M., \& HANSSON, R. O. Strategic self development for successful aging at 
work. International Journal of Aging and Human Development, v. 64, n. 4, p. 331-359, 2007. DOI: 10.2190/Q303-171U-7686-8517. Disponível em: https://journals.sagepub.com/doi/10.2190/Q303-171U-7686-8517. Acesso em: 01 fev. 2021.

ROBSON, S. M., et al. Successful aging: Criteria for aging well in the workplace. Journal of Career Development, v. 33, n. 2, p. 156-177, 2006. DOI:10.1177/0894845306292533. Disponível em: https://journals.sagepub.com/doi/10.1177/0894845306292533. Acesso em: 01 fev. 2021.

ROWE, J. W.; KAHN, R. L. Human aging: usual and successful. Science, v. 237, n. 4811, p. 143-149, 1987. DOI: 10.1126/science.3299702. Disponível em: https://science.sciencemag.org/content/237/4811/143.abstract?casa_token=0UpcIz1QMusAA AAA:d6vDKRKFofwJLEjqAVWLuAdX6yZVPozVwuExrOPFSiXOJoyEnycSiVSUcgJmceR LmQpPOxz7v3fg-g. Acesso em: 01 fev. 2021.

SANTOS, S. S. C. Concepções teórico-filosóficas sobre envelhecimento, velhice, idoso e enfermagem gerontogeriátrica. Rev. bras. enferm., v. 63, n. 6, p. 1035-1039, 2010. DOI: https://doi.org/10.1590/S0034-71672010000600025. Disponível em: http://www.scielo.br/scielo.php?script=sci_arttext\&pid=S003471672010000600025\&lng=en\&nrm=iso. Acesso em: 01 fev. 2021.

SARTES, L. M. A; SOUZA-FORMIGONI, M. L. O. Avanços na psicometria: da Teoria Clássica dos Testes à Teoria de Resposta ao Item. Psicol. Reflex. Crit., v. 26, n. 2, p. 241-250, 2013. https://doi.org/10.1590/S0102-79722013000200004. Disponível: http://www.scielo.br/scielo.php?script=sci_arttext\&pid=S0102-

79722013000200004\&lng=en\&nrm=iso. Acesso em: 01 fev. 2021.

SHI, W. H.; et al. The Status and Associated Factors of Successful Aging among Older Adults Residing in Longevity Areas in China. Biomedical and Environmental Sciences, v. 29, n. 5, p. 347-355, 2016. DOI: 10.3967/bes2016.045. Disponível em: https://PubMed.ncbi.nlm.nih.gov/27353709/. Acesso em: 01 fev. 2021.

SILVA, T. O. O bem-estar social no envelhecimento: estudo de caso em um centro de convivência em Brasília. 2019. 105 f. Dissertação (Mestrado em Gerontologia Social) Universidade Católica Portuguesa, Portugal, 2019.

SOUZA, A.C.; ALEXANDRE, N.M.C.; GUIRARDELLO, E.B. Propriedades psicométricas na avaliação de instrumentos: avaliação da confiabilidade e da validade. Epidemiologia e Serviços de Saúde, v. 26, n. 3, p.649-659, 2017.

SOUZA, J. J.; SALES, M. B. Tecnologias da Informação e Comunicação, smartphones e usuários idosos: uma revisão integrativa à luz das Teorias Sociológicas do Envelhecimento. Revista Kairós Gerontologia, v. 19, n. 4, p. 131-154, 2016. DOI: https://doi.org/10.23925/2176-901X.2016v19i4p131-154. Disponível em: https://revistas.pucsp.br/index.php/kairos/article/view/31957. Acesso em: 01 fev. 2021.

TEIXEIRA, S. M. O.; et al. Da velhice estigmatizada à dignidade na existência madura: novas perspectivas do envelhecer na Contemporaneidade. Estudos e Pesquisas em Psicologia, v. 16, n. $2, \quad$ p. 469-487, 2016. Disponível em: https://www.epublicacoes.uerj.br/index.php/revispsi/article/view/29179/20642. Acesso em: 01 fev. 2021. 
TOGINHO-FILHO, D. O.; ANDRELlO, A. C. Catálogo de Experimentos do Laboratório Integrado de Física Geral. Departamento de Física, Universidade Estadual de Londrina, 2010. Disponível em: http://www.uel.br/pessoal/inocente/pages/arquivos/03Conceitos\%20de\%20medidas\%20e\%20teoria\%20de\%20erros.pdf. Acesso em: 01 fev. 2021.

TUDGE, J.; ROSA, E. M. Bronfenbrenner's Ecological Theory. Wiley Online Library, 2020. DOI: https://doi.org/10.1002/9781119171492.wecad251. Disponível em: https://onlinelibrary.wiley.com/doi/abs/10.1002/9781119171492.wecad251. Acesso em: 01 fev. 2021.

VERAS, R. P. Guia dos Instrumentos de Avaliação Geriátrica [Recurso Eletrônico] - Rio de Janeiro: Unati/UERJ, 2019. 20 f. $\quad$ Disponível: https://www.unatiuerj.com.br/Guia\%20dos\%20instrumentos\%20Avaliacao\%20Geriatrica.pdf . Acesso em 26 fev. 2020.

VON S. H.; LEAL, I. Adjustment to Aging in Late Adulthood: A Systematic Review. International Journal of Gerontology, v. 8, n. 3, p. 108-113, 2014. DOI: https://doi.org/10.1016/j.ijge.2014.03.003. Disponível em: http://www.sciencedirect.com/science/article/pii/S1873959814000581. Acesso: 01 fev. 2021.

ZACHER, H.; RUDOLPH, C. W. Success Aging at Work and Beyond: A Review and Critical Perspective. Age Diversity in the Workplace, v. 17, Emerald Publishing Limited, p. 35-64, 2017. DOI: https://doi.org/10.1108/S1877-636120170000017004. Disponível em: https://www.emerald.com/insight/content/doi/10.1108/S1877-

636120170000017004/full/html. Acesso em: 01 fev. 2021. 